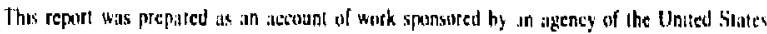

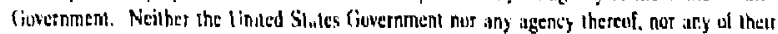

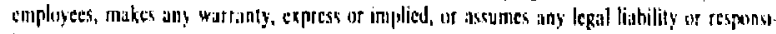

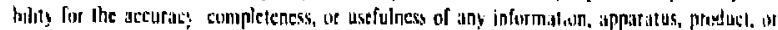
process disclosed, or represents that its use would not infriose rrivstely ow nod rights Refor

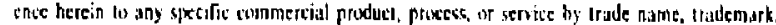

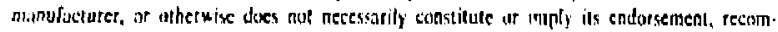
mendation. ut favoring by the Uniled Stales Ciuvernnent of any arency therest. The viens and opinions of autharx expecssed herein da nut neceswarily slate ar rellect thase of the liniled States Governatent ut uny agency theiced.

\title{
BEAM DIRECTOR DESIGN REPORT
}

\section{AUGUST 1986}

VOLUME II

UCRL--15901-V01.2

DE87 00B490

Prepared by:

Brobeck Corporation

1235 Tenth Street

Berkeley, California 94710

415-524-8664

Report Number 4500-316-R1

Francis C. Younger, Principal Investigator

Preparedfor:

Lawrence Livermore National Laboratory Livermore, California, USA

Under LLNL Subcontract 8652605 ,

U. S. Department of Energy Contract W-7405-ENG-48

Sponsored by:

PMS-405 Naval Sea Systems Command

Washington, $D C$ 


\section{TABLE OF CONTENTS - VOLUME II}

A. Funding Instruments $A-1$

B. Requests for Quotations B-1

C. Purchase Orders $6-1$

D. Drawings: Achromat D-1

E. Drawings: Vernier E-1

F. Magnetic Heasurements Reports, Lawrence Berkeley Laboratory F-1

G. Conceptual Desigil of an Electron Bean Deflector G-1

H. Beam Director Components for ATA Tests (Final Report) H-l 


\section{APPENDIX A. FUNOING INSTRUMENTS}

CONCEPTUAL DESIGN STUOY -- LBL

P.0. 4522410, 6-2-83

Change Order No. 1, 9-19-83

BEAM DIRECTOR COMPONENTS FOR ATA TESTS -- LLNL

Subcontract 2700700, Amendnent 11, 2-22-84

A-14

Subcontract 4368008 (Task II), 2-29-84

Subcontract 4368008, Amendment 2, (Task [I.C), 6-26-84

Telecon, verbal authorization to proceed, 2-11-85

Subcontract $6551805,5-6-85$

Subcontract 6551805, Amendment No. 1, 5-10-85 


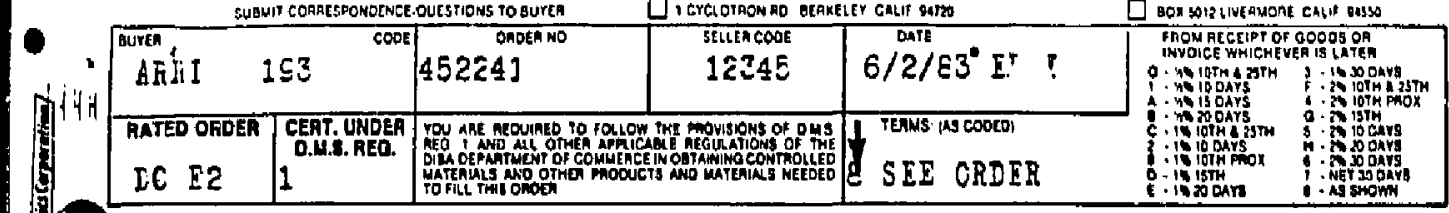

TO

TILIAN $M$. ERCEICE \& ASSOCIATES

1235 TENTE STEEIT

EIAREIEI, CL \$4710

AIIN: HLEREN FUREL

UNIVERSITY OF CALIFORNIA

SHIP TO

EII EOLY OF OÃLER

\section{PURCHASE ORDER}

FOA CONTRACT NO. W-7AO5-ENG. A

OR CONTAACT NO. DE.ACOJ-76SFOOOOS

WITH THE DEPARTMENT OF ENERGY

FOR RESALE - State Sales Tax should not bo chuiged, as the University holds State Sales Tax Permit C 135323

MAIL INVOICE IN DUPLICATE TO:

UNIVERSITY OF CALIFORNIA

LAYHENCE BERKELEY LABORATORY

Purchasa Ordas Admin|strulor
1 Cyclotron Aosd, Bulldino

callíainit mpto

payment procesting

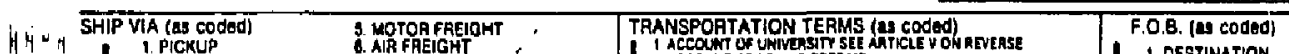

1 PICKUP

1 2 PARCEL POST

7. AR PAACEL, POST

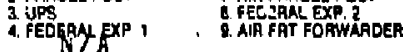

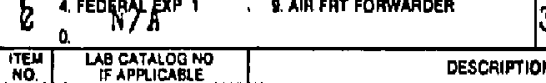

WILLIAM M. EROBICR \& $\triangle S S O C I A T I S$, RERDIN AND ON TEI REVERSE EEREOF

CALIET "SEIIEA", BGREIS TO FORNISE TC UNIVERFITY'S IAHRENCA

EFRKILI LAEORATORY, BINEIN SND IN ATTACEMENTS BEAETC CALLED

"UCIEI", TEE TOLLCWING IN STRICT ACCCRLANCE WITR THE TERYS,

CCNDITICNS, AND PRCVISICNS CF TEIS CRDER:

I. SCCPE OI WORX:

- -

SELIEA SBAII TORNISE AIL LABOB, MATERIALS ANE ITEMS NECESSARY

FCR THE COHCEFTOAL DESIGN CF BN ELECTRON BRAf DIRECTCR IN

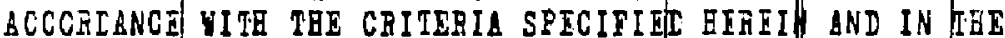

ATTACBED AFFENDIX A - SCCFI OF WOKR.

II. FAICF, $\triangle$ CCEPTANCT AND PAYMTNT:

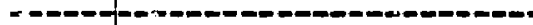

SELIER SHAII EERFORM TBE YOCRI IESCDIEEL HERE IN

FOR IEI FIR FIXIS PRICE CT...................\$47,783.06

- CONTINUEL CN FAGI

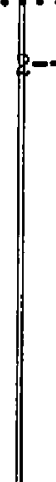

The Terms and Conditions On Tht Reverse Side Constitute A Part Of This Order

AL.23S4 (REV. Y/23) 7600-6128T

Authorized by

UNIVERSITY OF CALIFOANIA 


\begin{tabular}{|c|c|c|c|c|c|c|}
\hline \multirow{2}{*}{1} & & \multicolumn{2}{|c|}{ SUQUII CORRESPONOEGMEROUESTIONS TO DUYEA } & \multicolumn{2}{|c|}{ 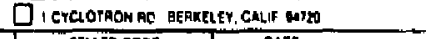 } & \\
\hline & BuYch & $1 \subseteq 3^{\prime}$ & $45 \approx 247^{\text {OAOE }}$ & $\begin{array}{l} \\
12345\end{array}$ & $\epsilon / 2 / E z^{*} I^{\cdots} P$ & 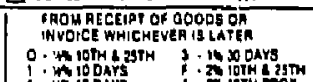 \\
\hline & $\begin{array}{l}\text { RATED OAOEA } \\
\text { IO EE }\end{array}$ & 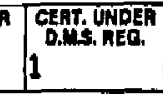 & 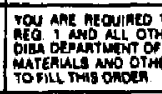 & & $\begin{array}{l}\text { TEAWS ALS COOED } \\
\text { SEI ORDER }\end{array}$ & 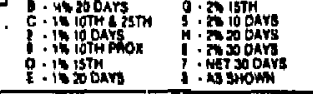 \\
\hline
\end{tabular}

TOIILIAM R. BRCFICK \& ASSCGIATES

UNIVERSITY OF CALIFORNIA

PURCHASE ORDER

FOR CONTRACT HO, W-74OS-ENG, 40

- OA CONTAACT NO DE-ACDJ-76SFO0098

WITH THE DEPAATMENT OF ENERGY

SHIP to

FAGE 2

FOR RESALE - Slate Sales Tau should not tos chargad, as tha Univeraly holds State Sales Tax Pormit C 125323

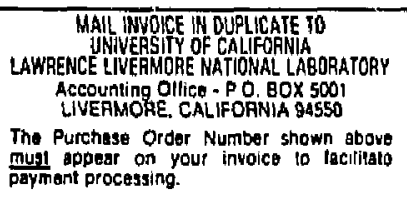

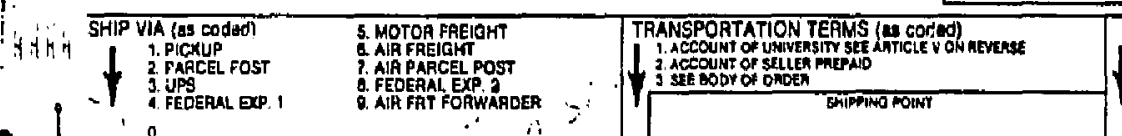

F O.B. (as codact)

1. Destination

2. SHIPPING POINT

4. FEDEAL EXP. 1 O. AIt FAT FORWAROER : :

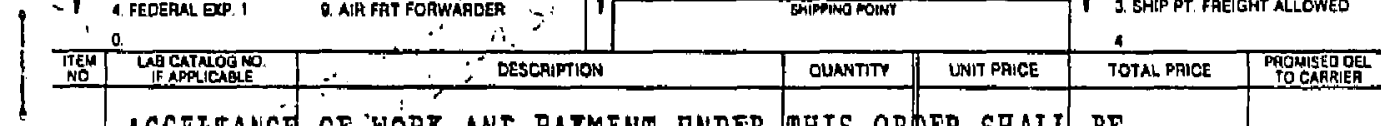

SCCEFTANCE CF WORT ANL PAIMENT ONDER THIS ORELR SHALL BE

EASEI CN SATISFACIORT COMPLIANCE WITE TEE TOLLONING:

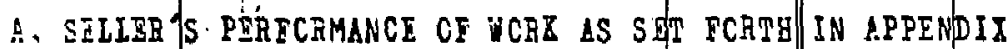

a IN CONSONANCE UITH EIGH FHOFISSIONAL ST NTARLS AS

- IETEEYIAIL BT IBL, AND

B. CCMPLEIICN CF TBE LEVEL CF EFFCRT SPECIFIPD IN PART III EEION, AND

C. COKFLIANCE WITB TES FEPCBIING EEQUITEYSNI SET FORTH IN AFFENDIX A - SCCFE OF WCRE.

SFLLIR MAT REQOEST ONIVERSITY TO MARE PROGRFS PATNFNTS HEFECNIER IN ACCCRDANCE UITB THE ATTACHEI FAPGRES PAI-

MENIS ARTICIS RI-2358 FF.

PROGEESS PAIK.ENTS IN TEE ANOONT OF SS PERCIN WILL BE KAIE ON TEL FASIS IOI TEE COST INCURREL FOR THE PERIO PEING EILLEL. EILIING NIII CONTAIN TES FOLLCWING DITAIL EY TASR: SAIARIES EY CATEGORI CF LAEOR, OTHER COSTS AND OVERETAD.

III. IEVEI CF REIORT:

IN THE FERTCRMANCF CF THE WCRE STATED EHRTIN SELLER AEREES IHAT IEL'S CONMACTING OPRICIR, IN BIS SOLF DISCRITION, NIA VERIFY BI

AOLIT AND FEQUIRE TEF FOLLONING IEVEIS OF EFFORT.

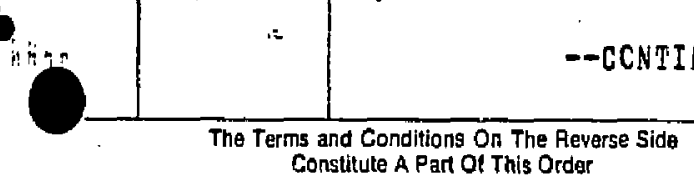

Al,-2354 (REY. Y/82) 7600-6486

Authorized by

H 


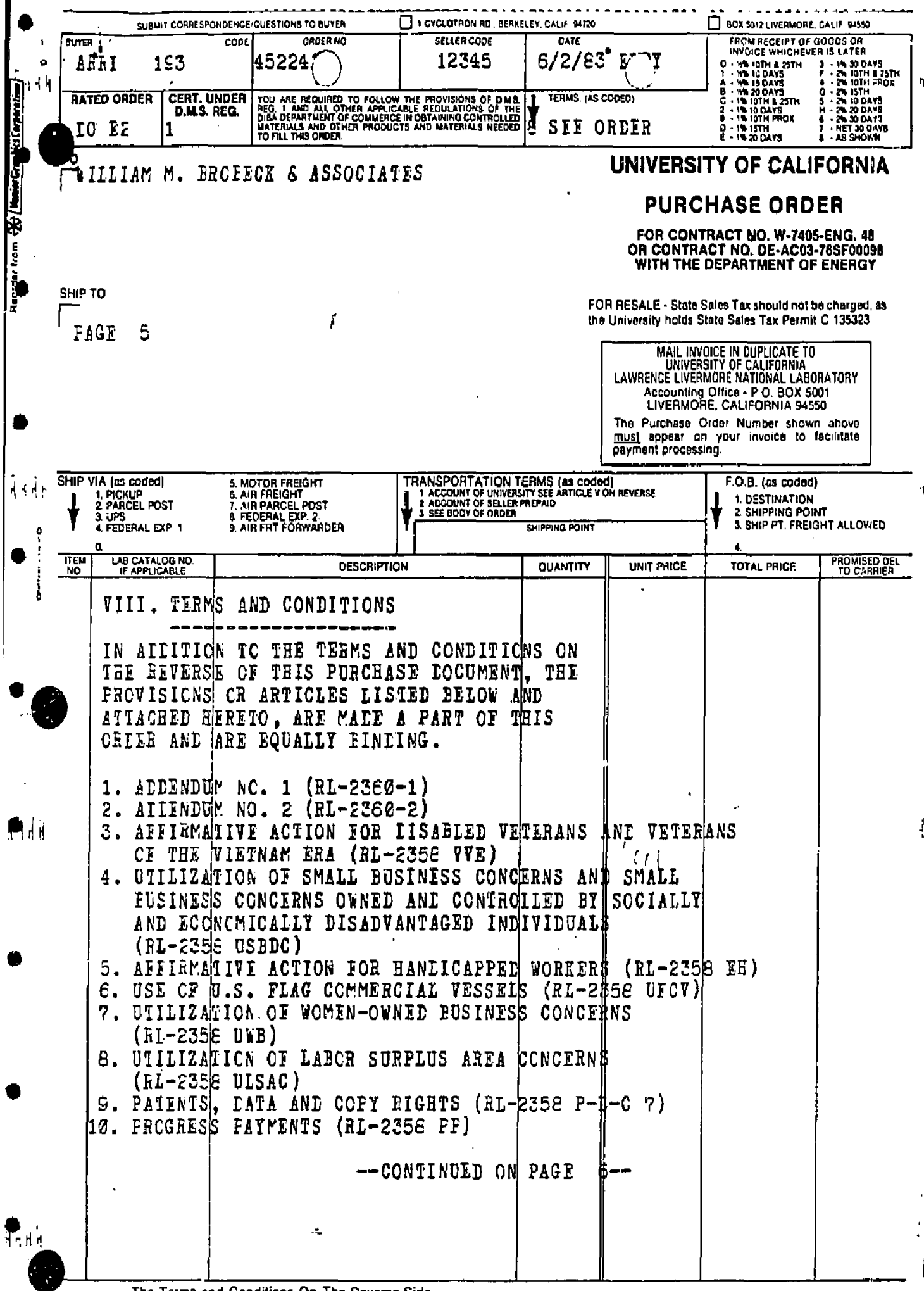

The Terms and Conditions On The Reverse Side Constivute A Part Of This Order

Aulthorized by 


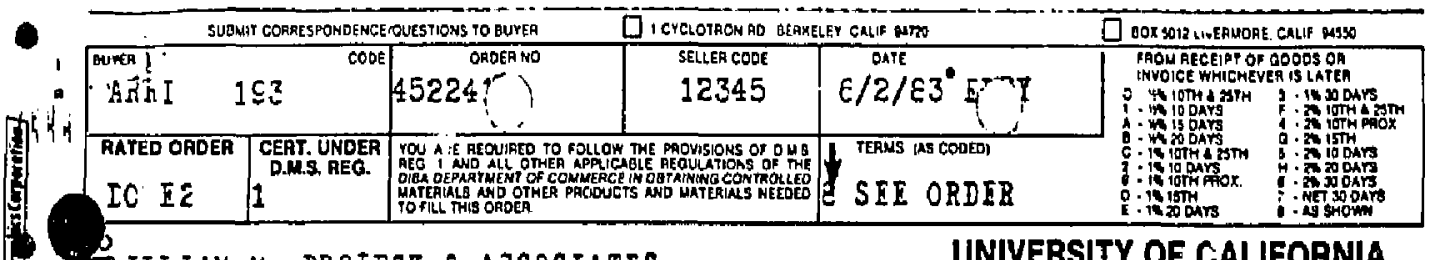

WIIIAM N. BRCEECR \& ASSOCIATES

UNIVERSITY OF CALIFORNIA

PURCHASË ORDER

FOA CONTRACT NO. W-7405-ENG, 45 OA CONTAACT NO. DE-ACO3.765FO009: WITH THE DEPAGTMENT OF ENEROY

SHIP TO

IAGE $\quad 6$

FOR RESALE - State Sales Tax should not be chargad, as the University holds State Sales Tax Permit C $13532 \mathrm{~J}$

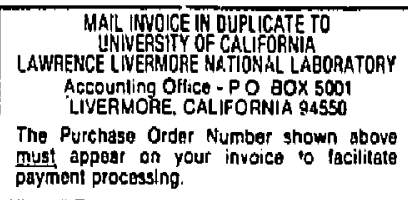

- SHIP VIA (as coded)

1. PICKUP

5 IHOTOA FAEIGHT

5. AA RAEIGHT

:EDERAL EXP. 2

3. UPS FEDEAA. EXP ।

9 MIR FAT FOAM ARDEA

\begin{tabular}{c|c} 
THEM \\
NO. \\
\hline TFB CATALOGNO \\
\hline
\end{tabular}

ACCIETEL:

B T:

DESCRIPTION

TRANSPOATATION TEAMS (BS COdOd)

IIIII:

DETE:

\section{HILIAM M. BROBECK \& ASSUCILTIS}

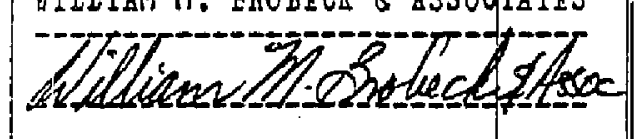
marren W. Eukel, Vice President June_3 3283

The Terms and Conditions On The Reverse Side Constitute A Part OI This Order

คL-2354 (REY, J/82) 7600-64207

F.0.B. (as codod)

I. DESTINATION 2 SHIPPING POINT I. SHIP PT FAEIOHT ALLOWED 4 \begin{tabular}{l|l}
\hline TOTAL, PRICE & PAOMISEO OEL \\
\hline
\end{tabular}

\begin{tabular}{|c|c|c|}
\hline UNIT PAICE & TQTAL, PRICE & PAOMISEO OEL \\
\hline
\end{tabular}


APPENDIX A - SCOPE OF WORK tO PURCHASE ORDER 4522410

between

THE REGENTS OF THE UNIVERSITY OF CALIFORNLA

and

WILLIAM M. BROBECR \& ASSOCIATES

I. INTRODUCTION

As approved and directed by the University, the Seller will undertake certain engineering work for the conceptual design of a electron bean director. It is estimated that approximately 860 man hours of technical effort will be required to complete this program. This engineering work is io be clogely coordinated with the scientific work being done by LBL ataff members on the same project.

\section{PURPOSE}

The overall objective of the proposed effort is to perform conceptual design engineering work leading to a final report that sets forth a conceptual design for an electron beam director and indentifies segments needing research and development.

III. TECHNICAL SCOPE OF WORK

The Seller shall pisform the following tasks:

TASR 1 Conceptual Design

Select suitable configurations and perform engineering calculations establishing conceptual designs for the various segments of an electron bear director system including:

- Magnet lattice

- Vacuum ro'ary joints

- Vacuun or air transicion

- Verrier steering system

- Mechanical mount and slewing system

- Radiation effects

- Estimates of size, weight and cost 


\section{TASK 2 Liaison}

The 3eller shall weet with LBL project stafi Bi-Monthly so that the engineering wort hereunder is coordinated with that being pertormed by LBL staff members.

\section{TASK 3 Final Report}

The Seller shall prepare and submit drafts of those portions of the Final Report that pertain to the conceptual degign engineering work undertaken hereunder by August 31, 1983. After review, a final draft of said portions of the Final Report shall be submitted by September 30, 1983.

\section{IV, REPORT SUBMITTALS AND DELIVERABLES}

The reports and deliverables requized are those described in Section III, Taskg 2 and 3 .

Two (2) copies of the above reports are to be addressed and submitred to:

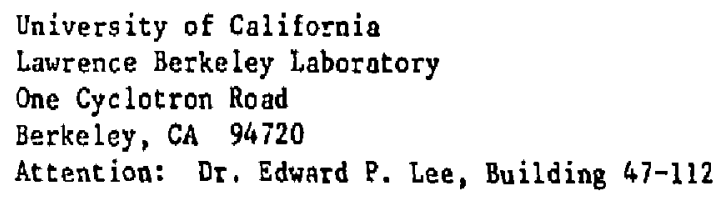

One copy of the transwittal letter for each of these reports shall be furnished to the Purchase Order Administrator addresged as follows:

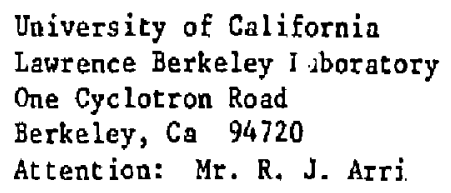

The Seller shall not distribute reports of work, drawings, specificatiuns, etc. under this purchase order to any individual or organization other chan those indicated above without the prior written approval of the Purchase Order Adrinistrator. 


\section{LIAISON}

A. University's Purchase Order Administrator is Mr. R. J. Arri, or his designee or succescor. He will assure compliance with the terms and conditions of this order, process any contractual approvals required and monitor expenditures hereunder. He shall also be responsible for review of all invoices submitted under the progresa payments provisions to assure compliance with the order requirements.

B. University's Technical Director under this order is Dr. Edward P. Lee, his successor or designee. He will be responsible for monitoring technical performance under this order, approving the form and content of technical reports and accepting the final task reports.

\section{ASSIGNMENT OF PERSONNEI.}

Any substitution, resssigarent, replacement or other change involving Seller's key technical personnel assigned to this work, shall be made only with persons of equal abilities and qualifications. Any such change shall be subject to prior University approval. 


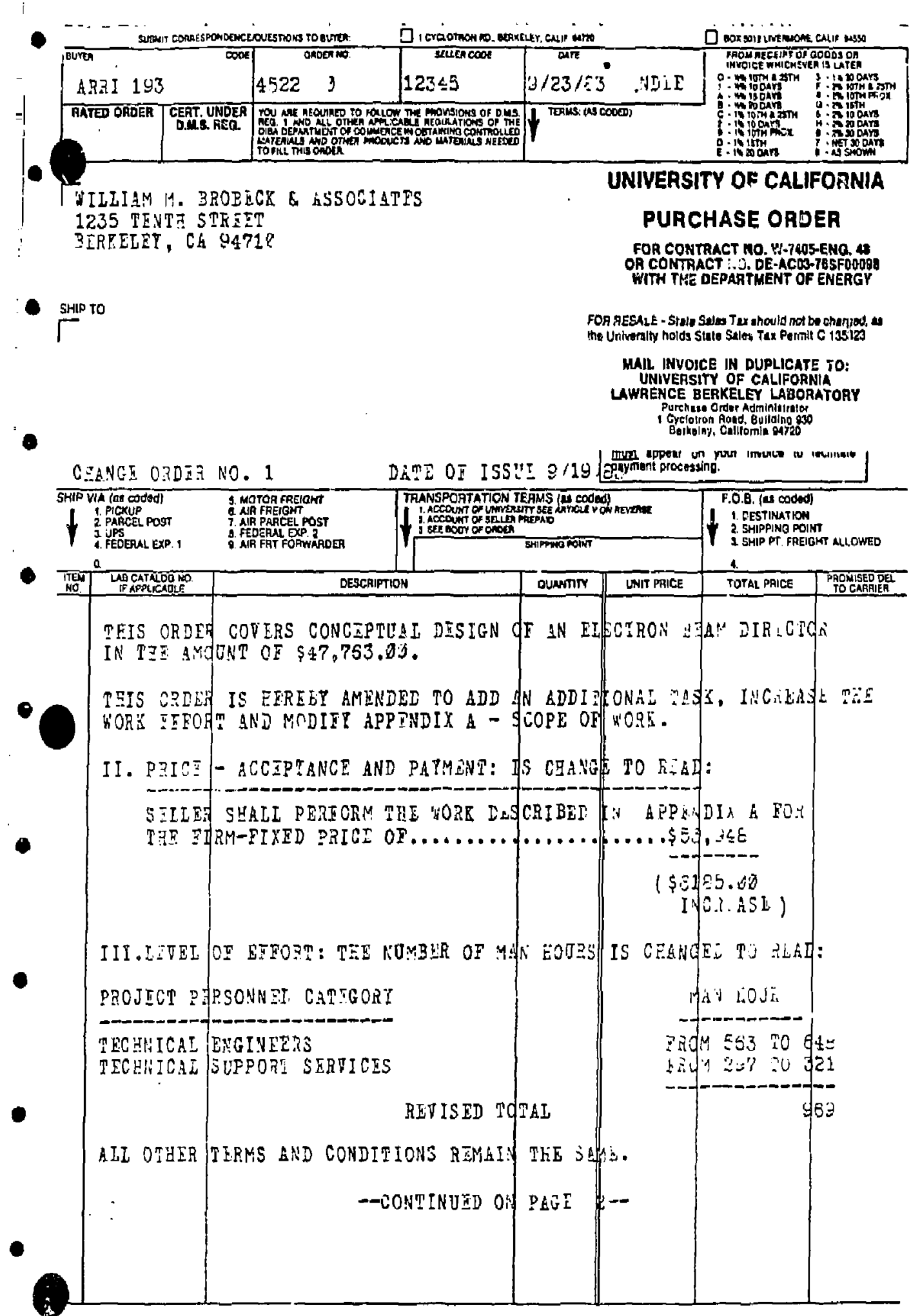

1) Authorized by 


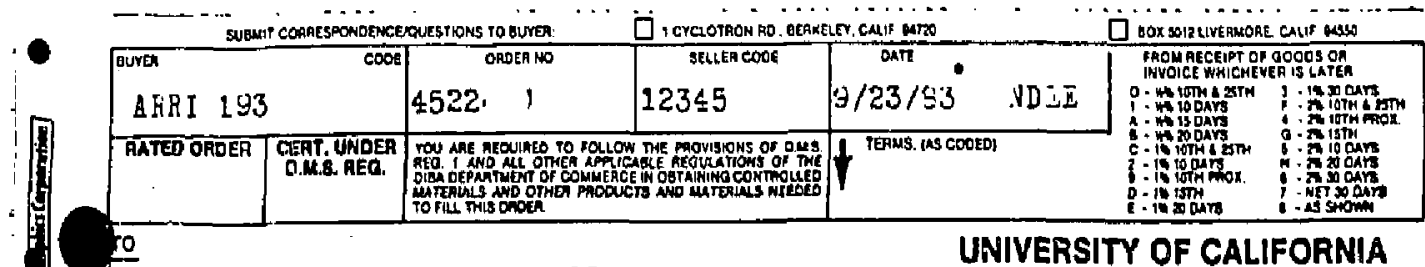

TILLIAM M. BHOBICE \& ASSOCIATES

UNIVERSITY OF CALIFORNIA

\section{PURCHASE ORDER}

FOA CONTRACT,ND. W-7405-ENO, 4

OA CONTAAGT NO, DE-ACOJ-T6SFDDDQA

WITH THE DEDAR TIMENT OF ENERGY

SHIP TO

FOR AESALE - SIIte Sula fex thould nol be chamed, th the Untwerity holds Siate Sales Tex Pormll C 125323

\section{PSGE 2}

\begin{tabular}{|c|}
\hline 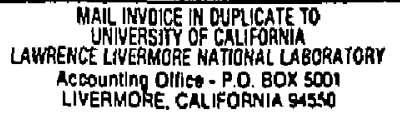 \\
\hline 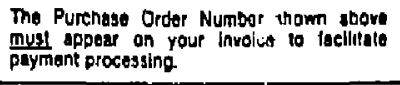 \\
\hline
\end{tabular}

SHIP VIA (E: avdod)

1. PhCKuP
2 PARCE POST

3. NOTOR FAEIGMT

- AIA FREIGHT

7. AIA PAACEL POST

4. Fengal : XP:

a. FEDEAR EPP.

d)

paymant processing.

AL 2354 (REV, J82) $7600-64246$ 
FIRST MODIFICATION TO APPENDIX A - SCOPE UF WORK

to

PURCHASE ORDER 4522410

with

WILLIAM M. BROBECK AND ASSOCIATES

\section{INTRODUCTION}

This First Modification to Appendix A - Scope of Work to Purchase Order 4522410 issued to provide contract soverage for additional work to be per formed.

\section{PURPOSE}

The purpose of this first Modification is to add an additional work task under this order.

CHANGES

Appendix A - Scope of Work is modified by mutual agreement of the parties as Eollows:

TECHNICAL SCOPE OF WOR: - is changed to read:

Trak 4 is added as followa:

"TASK 4 consist of planning how the concepts and configurations $\vdots$ developed in the following taska can be carried forward to a succesaful demonstration on ATA at Lawrence Livermore National Laboratory.

- Magnet Lattice

- Vacuum Rotary Joints

- Vacuum-to-Air Transition

- Vernier Steering System'

- Mechanical Mount and Slewing System

- Radiation Effects

- Estimates of Size, Weight and cost"

All other provisions of Appendix A - Scope of work to Purchase order: 4522410 remain unchanged. 


\section{ELEVENTH AMENDMENT}

to

SUBCONTRACT 2700700

between

THE REGENTS OF THE UNIVERSITY OF CALIFORNIA

and

BROBECK CORPORATION

INTRODUCTION

This Eleventh Amendment to Subcontract 2700700 is entered into by and between The Regents of the University of California, hereinafter called "University", and Brobeck Corporation, hereinafter called "Subcontractor".

PURPOSE

The purpose of this Eleventh Amendment is to recognize a change in Subcontractor's name; to provide for additional Tasks under existing Part A - Organic Materials and to add a new Part E - Beam Director; to increase the level of effort; to increase the funding; and to extend the Subcontract term through February 29, 1984.

HOW, THEREFORE, effective upon Subcontractor s receipt of a fully executed Amendment, Subcontract 2700700 between the University and the Subcontractor is hereby anended by mutual agreement of the parties as follows:

CHANGE OF NAME

This Amendment acknowledges that University and Subcontractor have executed a CHANGE OF NAME AGREEMENT on November 22, 1983, recognizing the Subcontractor has changed its corporate name to BROBECK CORPORAIION.

ARTICLE II - TERM - is changed, in part, as follows:

Delete "and shall continue through December 31, 1983", and substitute in lieu thereof "and shall continue through February 29, 1984".

ARTICLE III - ESTIMATE OF COST - Paragraph A. In itial Estimate of cost and fixed fee: delete as written and substitute therefor:

"The presently estimated cost of work under this Subcontract is THREE HURDRED SEVENTY-SEVEN THOUSAND THREE HUNDRED FIVE AND NO/100 DOLLARS $(\$ 37), 305.00)$, exclusive of Subcontractor's fixed fee. Subcontractor's fixed fee is THIRTY-SEVEN THOUSAND SEVEN HUNDRED TWENTY-TWO AND NO/100 DOLARS $(\$ 37,722.00)$. The aggregate of presently estimated cost and fixed fee is FOUR HUNORED FIFTEEN THOUSANO THENTY-SEVEN AND NO/100 DQLARS $(\$ 415,027.00) . "$ 
APPENDIX A - REIMBURSEMENT OF COSTS is hereby revised to add Paragraph C.3, as follows:

"C.3. Notwithstanding Paragraphs C.I and C.2, above, the parties hereío agree that University shall not be obligated to pay any amount for General and Administrative Expenses (G\&A) exceeding $30.0 \%$ of total direct cost and Overhead, for the period commencing January $1,1984 . "$

APPENDIX $\vec{B}$ - SCOPE OF WORK, is hereby revised as follows:

Paragraph III. TECHNICAL SCOPE OF WORK, is revised as follows:

Part A - Organic Materials, add the following:

"Task XII

"Initiate efforts to extend RDUCT to include additional procedures for transition computation including "microscopic" computations of internal cell heat transfer."

"Task XIII

"Provide support analyses in the area of weapons vulnerability, as requested and under the technical direction of the LLML Technical Coordinator, or his designee."

Add the following new task: -

\section{"Part E - Beam Director}

"Initiate planning and preliminary design of three elements of the electron beam director system and detail design of the Vernier Steering and Achromat for the LLNL Rdvanced Test Accelerator (ATA) project.

"The prioritized elements are:

1. Vernier Steering

2. Achromat

3. Vacuum-to-Air Rotating Window

"Work shall be performed as approved and directed by the University's Technical Coordinator, and generally as described in the WORK STATEMENT (4 pages) contained in Subcontractor's Proposal No. 8400-260-20A dated February 10, 1984."

"The Final Report for this Part $E$ shall be an informal summary of work performed, in form and content acceptable to the University's Technical Coord inator."

Paragraph IV. LEVEL OF EFFORT; is modified in its entirety to read as follows: 
"The allocation of effort under Subcontract is estimated to be:

Type of Personne]

PAKT A

\section{Engineer 15 \\ Engineer 16}

PART B

Engineer 16

Engineer 15

Engineer 12

Engineer 11

Engineer Associate 7

PART C

Engineer 16

PART D

Engineer 12

PART E

\section{Engineer 16 \\ Engineer Associate 7 \\ Engineer Associate 5}

$$
\text { Leve I-of-Effort }
$$

(Man-Hours, Cumul at ive)

1,412

2,330 (increase 174)

320

861

470

610

446

35

8

$$
\begin{aligned}
& 670 \text { (added) } \\
& 358 \text { (added) } \\
& 2401 \text { (added) } \\
& \hline 7760
\end{aligned}
$$

Part VI. COOROINATION AND ADMINISTRATION, is revised to ado the following:

"The University's Technical Ccordinator for Part E - Bean Diractor, is Coleman Johnson, or his designee."

All other terms and conditions of Subcontract 2700700 shell remain in full force and effect except as amended herein.

IN WITNESS WHEREOF, the parties have executed this Eleventh Amendment.

- accepted:

BROBECK CORPORATION
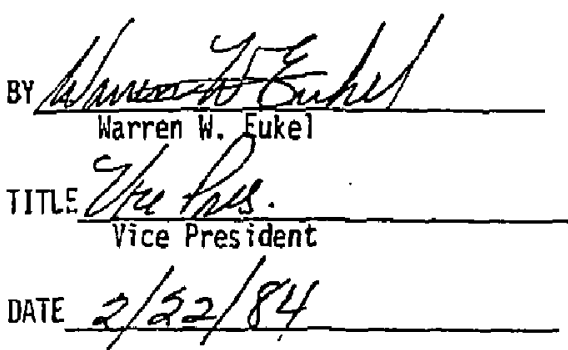

11 th Amendment

S/C 2700700

2/84 (2233N:30i)
AUTHORIZED:

THE REGENTS OF TH

UNIVERSITY IFICA HF OROIA

BY.

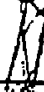

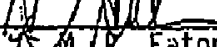

TITLE Deputy Contracts Manager

Lawrence Livermor National Laboratory

DATE

A-16

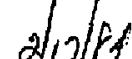


THE REGENTS OF THE UNIVERSITY OF CALIFORNIA

and

BROBECK CORPORATION

\section{INTROOUCTION}

This cost Plus Fixed Fee Subcontract is made and entered into by and between The Regents of the University of California, hereinafter called the "University", and Broberk Corporation, hereinafter called the "Subcontractor".

The Regents of the University of California, a California corporation, have entered into a contract, W-7405-ENG-48 with the United States Government, hereinafter called "Government", represented by the Department of Energy, hereinafter called "DOE", for the performance of certain energy research and development work at the University of California Lawrence Livermure National Laboratory. This Subcontract is entered into in furtherance of the performance of the work provided for in the prime contract.

In accepting this Subcontract, the Subcontractor agrees to perform the Subcontract work in accordance with the following documents which collectively constitute the Subcontract and are attached hereto and by this reference made a part hereof:

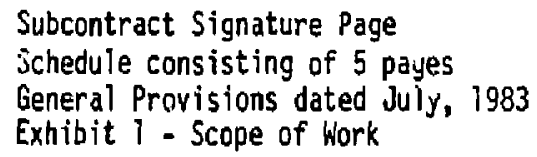

(Revised 6/83)

ACCEPTED: BROBECK CORPORATION

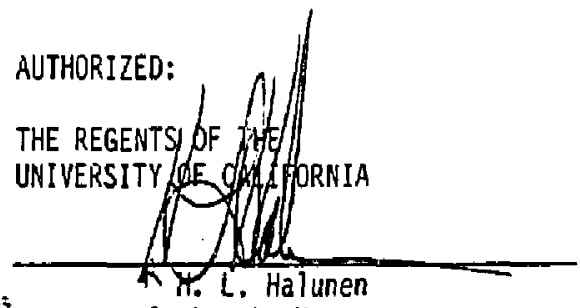

Title: Vice President

Contracts Manager

Date: February 29,1984

Lawrence Livermore National Laboratory

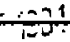


The Subcontractor shall furnish the necessary facilities, supplies, equipment and personnel, except such as are furnished by the University or the Government, to conduct certain work generally described as a) Task 1 Analysis of Weapons System Explosives Component Vulnerability, and b) Task II - ATA Beam Director, to be performed by the Subcontractor at locations approved by the University and Subcontractor's facility at Berkeley California the nature and extent of which are more specifically described in EXHIBIT 1 - STATEMENT OF WORK hereto, and shall also deliver such materials, products, supplies and incidental se:vices and reports as may be set forth in sald EXHIBIT $\mathrm{I}$.

The University and DOE sirall have the right to inspect Government property and the work and activities of the Subcontractor under this Subcontract at such time and in such manner as they shall deem appropriate. The Subcontractor shall include in all subcontracts and purchase orders under this Subcontract involving research and development or fabrication, or where costs incurred are a factor in defermining the amount payable to the vendor, a similar provision making this paragraph applicable to the Subcontractor or vendor.

(Revised 6/83)

\section{ARTICLE II - TERM}

Unless completely performed prior thereto or sooner terminated in accordance with CLAUSE 14 - TERMINATION, the work described in ARTICLE I SCOPE OF WORK, shall begin March 1, 1984 and shall continue through December 31, 1984 which is the current period of time estimated as necessary for coripletion of said work, provided that the term of this Subcontract shall continue for such period of time as required to comply with the reporting and close out procedures of this Subcontract. Neither party guarantees the aforestated estimate of time and work under this Subcontract and, except as necessary to comply with reporting and close out procedures, the term shall cease on the last date mentioned unless the University by writter, notice to Subcontractor extends, at its sole option, such period for such time or times as it considers appropriate for completion of the work described in ARTICLE I - SCOPE OF WORK. Sus: an extension of time will not be a basis for a claim of additional iee by Subcontractor.

(Revised 2/83) 
A. Initial Estimate of Cost and Fixed Fee

The presently estimated cost of the work under this Subcontract is $\$ 96,257.00$ exclusive of the Subcontractor's fixed fee. The Subcontractor's fixed fee is $\$ 9,618.00$. The aggregate of presently estimated cost and fixed fee is $\$ 105,875.00$.

B. Revised Estimate of cost

(Task II: $52,310.00$ )

The preseritly estimated rost of the work under this Subcontract may be increased or decreased by written agreement of the parties.

C. Limit on Total Amount of Allowable Costs

Payments on account of costs shall not in the aggregate at any time exceed the amount of estimated costs specified in the Subcontract.

D. Notice of Costs Approaching Subcontract Amount - Subcontractor Excused Pending Increase

Whenever the Subcontractor has reason to believe that the total costs of the work under this subcontract, exclusive of fixed fee, will exceed the estimated cost of the work then in effect, the Subcontractor shall promptly notify the University in writing. The Subcontractor shall also notify the University in writing when the aggregate of expenditures and outstanding comnitments allowable under this Subcontract, exclucing fixed fee, is equal to $75 \%$ for such other percentage as the University may from time to time establish by notice to the Subcontractor) of the estimated cost of the work then in effect. When such expenditures and outstanding commitments, excluding fixed fee, equal $100 \%$ of such amount, the Subcontractor shall make no further comitments or expenditures (except to meet existing comitments) and shall be excused from further performance of the work unless and until the University shall increase the estimated cost of the work in accordance with Paragraph 8 . above.

E. The University's Right to Terminate not Affected

The giving of any notice by either party under this ARTICLE III shall not be construed to waive or impair any right of the University to terminate the subcontract under the provisions of iLAUSE 14 . TERMINATION.

F. iost Information

The Subcontractor shall maintain current cost information adequate to reflect the cost of performiting the work under this Subcontract at aly times while the work is in progress and shall prepare and furnish to the University such written estimates of cost and information in support thereof as the University my request. 


\section{G. Correctness of Est imates not Guaranteed}

Neither the University nor the Subcontractor guarantees the correctness of any estimate of cost or estimate of time for the perfermance of the work under this Subconiract, and there shall be no adjustment in the amount of the Subcontractor's fixed fee by reason of errors in the computation of estimates or differences between such estimates and the actual cost or time for the performance of the work.

(Revised 2/83)

\section{ARTICLE IV - C.ONSIOERATION}

Payment for allowable casts, reimbursable in accordance with ARTICLE $V$ REIMBURSEMENT OF COSTS, and of the fixed fee set forth in ARTICLE III ESTIMATES AND FIXED FEE, shall constitute complete compensation for Subcontractor's services, inciuding profit and all items or kinds of expenses not allowible under the terms of this Subcontract.

(Revised 2/83)

\section{ARTICLE $V$ - REIMBURSEMENT OF COSTS}

A. The allowable cost of performing the work under this Subcontract shall be the costs and expenses (less applicable income and other credits) that are actually incurred by the Subcontractor, are applicable and properly chargeable, either as directly incident or as allocable through appropriate distribution or apportionment, to the performance of the Subcontract in accordance with its kerms and are determined to be allowable pursuant to this paragraph. The determination of the allowability of cost hereunder shall be based on: 1) reasonableness, including the exercise of prudent business judgment; 2) consistent application of generally accepted accounting principles; and 3) recognition of all exclusions and limitations set forth in this ARTICLE $V$, or elsewhere in this Subcontract as to types or amounts of items of cost. This Subcontract recognizes that audit hereunder will be in accoriance with this Subcontract and Contract cost principles and procedures of DOE (DOE PR 9-15.50) in effect on the date of this Subcontract, as supplemented by Subpart $1-15.2$ of the Federal Procurement Regulations (4) CFR 1-15.2) and in DOE PK 9-15.205-3 "Bidding Cost" and 9-15.205-35 "Independent Research and Development (IR\&D) and Bid and Proposa] (B\&P) Costs". The audit might be performed by the Government Agency having the preponderant work load. However, DOE reserves the right to conduct its own audit of expenditures under this subcoritract.

(Revised 6/83) 
8. OVERHEAD

1. Pending final audit and detarmination of overhead, the Subcontractor stiail be paid a provisional overhead rate, as approved by the University from time to time and authorized in writing by the University. Such provisional payments will be adjusted to the actual overhead costs and expenses determined as set forth in this ARTICLE $V$ and principles herein established.

2. When actual reimbursable overhead costs have been determined pursuant to this Subcontract, adjustment shall be made for the difference between the sum to be determined and the sum of the provisional payments made under Paragraph 1,, above.

3. Notwithstanding Paragraphs B.1 and B.2 above, the parties hereto agree that University shall not be obligated to pay any amount for General and Administrative Expenses (G\&A) exceeding $30.0 \%$ of tota) direct costs and Overhead, for the period commencing March 1, 1984.

(Revised 2/83)

MKIICLE VI - RIGHTS TO PROPOSAL DATA

Except for technical data contained in pages (None) of the Seller's proposals dated February 10, 1984 which are asserted by the Seller as being proprietary data, it is agreed that as a condition of the award of this subcontract, and notwithstanding the provisions of any notice appearing on the proposal, the University and Government shall nave the right to use, duplicate and disclose and have others do so for any purpose whatsoever, the technical data contained in the proposal upon which this order is based.

(Revised 2/83)

\section{ARTICLE VII - GENERAL PROVISIONS}

A. The Subcontractor shall comply with the CLAUSES set forth in the Gereral Provisions dated July 1983 as may be altered or modified hereunder and in the ARTICLE VIII - ALTERATIONS and ADDITIONS.

B. CLAUSE 31, paragraphs A.1, A.2, Authorization and Consent (page no. 26 and 27), shall or shall not be appiicable to this Subcontract as follows:

\footnotetext{
Paragraph $A_{.} l$ is $[X]$ is not [ ] applicable to this Succontract. Paragraph $A .2$ is [ ] is not [X] applicable to this subconiract.
} 
C. CLAUSE 37, paragraphs B. 7, B.2, Patent indemnity (page no. 27), shali or shall not be applicable to this Subcontract as follows:

Paragraph $B .1$ is [X] is not [ ] applicable to this Subcontract.

Paragraph 8.2 is [ ] is not [X] applicable to this Subcontract.

D. CLAUSE 31, paragraphs $0.1,0.2$, Patent Rights (page no, 28 or 36), shall be applicable to this Subcontract as follows:

Paragraph D I PATENT RIGHTS (LONG FORM) is [ ] is not [X] applicable tc this Subcontract.

Paragraph 0.2 PATENT RIGHTS (SMALL BUSINESS FIRMS AND NONPROFIT ORGANIZATIONS, MARCH 1982) is [X] is not [ ] applicable to this Subcontract.

E. CLAUSE 31, paragraph G, Rights in Technical Data, subparagraphs $G$ (page no.41) and $H$ (page no.44) shall or shall not be applicable to this Subcontract as follows:

Subparagraph $G$ is [X] is not [ ] applicable to this subcontract.

Subparagraph $H$ is [ ] is not [X] applicable to this Subcontract.

(Revised 6/83)

\section{ARTICLE VIII - ALTERRTIONS AND ADOITIONS}

The following optional clause is hereby incorporated into and made a part of this Subcontract.

Clause 35 Cost or Pricing Datz. (page 48)

In addition, the foilowing Articie is hereby incorporated into and made a part of this Subcontract:

ARTICLE 38 - Foreign Ownership, Control, or Influence Over Contractor, Form $L L$

Revised 2/83)

Schedule 
EXHIBIT 1 - SCOPE OF WORK

to

SUBCONTRACT 4368005

between

THE REGENTS OF THE UNIVERSITY OF CALIFORNIA

and

BROBECK CORPORATION

I. INTRODULTION

As approved and directed by the University, Subcontractor shall provide technical and professional effort directed toward the studies specified in Part III - Technical Scope of Work.

As provided in CLAUSE 3 - CHANGES, the University may direct, in writing, changes within this Scope of Work.

\section{PURPOSE}

The purpose of this work is a) to provide design and analysis for two phase hydrodynamic calculations, and to provide weapons vulnerability studies (Task I), and b) to provide planning and design engineering for an electron beam director vernier, achromat and window (Task II).

III. TECHNICAL SCOPE OF WORK

As approved or directed by the University, Sulcontractor shall furnish all necessary personnel, materials and facilities, except as. may be provided for elsewhere herein, to conduct the investigations and Tasks described below:

\section{IASK I - CHEMISTRY DEPARTMENT SUPPORT}

- I. A Engineering Design and Analysis for Two Phase Hydrodynamic Calculations:

Initiate efforts to extend ROUCT to inciude additional procedures for transition computation including "microscopic" computations of internal cell heat transfer. (Note: this is a continuation of efforts initiated under Part A-Task XI I of Subcontract 2700700.) 


\section{I.B Weapons Vulnerability Studies:}

Provide support anaylses in the area of weapons vulnerability, as reauested and under the technical direction of the LLM Technical Representative. (Note: this is a continuation of efforts initiated under Part A - Task XIII of Subcontract 2700700.)

\section{TASK II - ADVANCED TEST ACCELERRTOR (ATA) SUPPORT}

\section{I1.A Bean Director:}

Complete the planning and preliminary design of three elenents of the electron beam director system and detail design of the Vernier Steering and Achromat for the LLNL Advanced Test Accelerator (ATA) project.

The prioritized elements are:

1. Vernier Steering

2. Achromat

3 Vacuum-ta-Air Rotating Hindow

Work shall be performed as approved and directed by the University's Technical Representative, and generally as descirbed in the WORK STATEMENT (4 pages) contained in Subcontractor's Proposal No. 8400-260-20A dated February 10, 1984. (Note: this is a continuation of efforts initiated under Part E- Beam Director of Subcontract 2700700.)

IV. LEVEL OF EFFORT

The distribution and allocation of technical and professional effort under this Subcontract shall be approximately as follows:

TYPE OF PERSONNEL

ask I

Chemistry Support

Engineer 16

Engineering Associate 7
MAN HOURS (ESTIMATED)

Jask II

ATA Support

699

83

V. REPORTS

Subcontractor shall prepare and submit the following reports to the " Universitys. 
A. Financial Reports (Type A) - sha?l be submitted by the fifteenth of each month and shall include all costs incurred during the preceding month and outstanding comitments to the end of the month. In the event actual cost data is unavailable, Subcontractor's monthly financial report shall conta'in estimated expenditures, identified as such. Data shall be segregated and indentified by Task. -

B. Monthly Progress Reports (Type B) - shall ye submitted by the fifteenth of each month and may be informal letter sumaries in a format approved by the University's Technical Representative. These reports should contain a description of work performed during the month reported and work planned for the succeeding mionth. Data shall be segregated and indentified by Task. (Subcontractor may elect to submit separate reports for each Task).

C. Final Report (Type C) - shali be submitted upon completion of each Task and contain a comprehens ive summary of all work results and conclusions. The form and content of Type $C$ Reports shall be acceptable to the University's Technical Representative. If so requested, a draft copy of the Report shall be submitted to the University's Technical Representative for approval prior to final typing.

D. Distribution of Reports

Reports shall be separately addressed and trinsmitted to:

University of California

Lawrence Livermore National Labolatory

P. 0 . Box 5012

Livermore, CA 94550

Attention: (Intended Recipient)

Type of Report

$A$ and $B$

$A$ and $B$

c
No. of Copies

$$
1
$$

As Requested

As Requested
Recipient

Diane Melendez

Technical

Representative for

Each Respective Task

$0 \mathrm{i}$ ane Melendez

Subcontractor shall not distribute reports of work under this Subcontract to any individual or organization other than those indicated above or an authorized representative of the U. S. Department of Energy withcut prior written approval of the Cortract Administrator. 
E. Interim Reports

If is understood that there will be other information exchanged between the parties from time to time. These data may be exchanged directly between the parties concerned; formal reporting and distribution is not required in these cases.

- VI. COORDINATLON AND ADMINISTRATION

The University's Technical Representative(s) under this Subcontract as designated below, or their designee(s), shall represent the University in matters relating to the technical performance of the Scope of Work described herein. During the established term of this Subcontract, the Technical Representatives will interpret the technical requirements of the Scope of Hork and will determine the emphas is and direction of the Subcontractor in the conduct of the work within the level and allocation of effort established herein. All other matters relating to the performance of this Subcontract are reserved to the Contract Administrator. Provided, however, any technical direction which will affect the estimated cost or time for performance of this work shall require prior formal amendment of this Subcontract or prior written direction from the Procurement Manager, or his dasignee, as provided in CLAUSE 3 - C.HANGES of the Terms and Conditions. Technical direction resulting in work outside the general Scope of Work requires prior formal amendment of this Subcontract.

$\begin{array}{lc}\text { Task } & \text { Technical Representat } \\ \text { Task I } & \text { E. Lee } \\ \text { Tas' II } & \text { Coleman Johnson }\end{array}$

The University's Contract Administrator is Diane Melendez, or her designee. All matters relating to the interpretation and administration of this Subcontract shall be conducted through the Contract Administrator. Subcontractor will direct all notices and requests for approval to the Contract Administrator; and any notice or approval from the University to the Subcontractor will be issued by the Contract Administrator.

VII. ASSIGNMENT OF PERSONMEL

It is understood and agreed that Subcontractor's key technical personnel assigned to this work shall not be reassigned or replaced without prior University approval, except where such circumstances are beyond the reasonatipe control of the Subcontractor. 


\section{HEALTH, SAFETY AND FIRE PROTECTION}

The Subcontractor shall take all reasonable precautions in the performance of the work under this Subcontract to protect the health and safety of employees and menbers of the public and to minimize danger from all hazards to life and property, and shall comply with

- all pertinent health, safety, and fire protection regulations and requirements (including reporting requirements) of the University and DOF communicated to the Subcontractor.

In the event the Subcontractor fails to comply with said regulations or requirements of the University or the $D O E$, the University may without prejudice to any other legal or contractual rights of the University, issue an order stopping all or any part of the work; thereafter, a start order for the resumption of work may be issued at the discretion of the University. 
SECOND AMENDMENT

to

SUBCONTRACT 4368005

between

THE REGENTS OF THE UNIVERSITY OF CALIFORNIA

and

BROBECK CORPORATION

\section{INTRODUIT,TION}

This Second Amendment to Subcontract 4368005 is entered into by and between The Regents of the University of California, hereinafter called "University", and Brobeck Corporation, hereinafter called "Subcontractor".

PURPOSE

The purpose of this Second Amendment to Subcontract 4368005 is to incorporate the following changed documents into this Subcontract.

NOW, THEREFGRE, by mutual agreement of the parties, the following Subcontract documents attached hereto are made a part of this Subcontract:

Second Modification to Schedule

Second Modification to Exhibit I

All other terms, conditions and provision of Subcontract 4368005 shall remain in full force and effect, except as amended herein.

in witness whereof, effect ive upon Subcontractor's signature acceptance of this Second Amendment, Subcontract 4368005 is hereby amended by mutual agreement of the parties.

ACCEPTED:

BROBECK CORPORATION

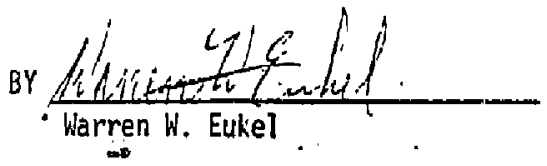

TITLE Vice President

DATE June 26, 1984
AUTHORIZED:

THE WE:GENTS OF THE

UNIVERSITY OF CALIFORNIA

BY

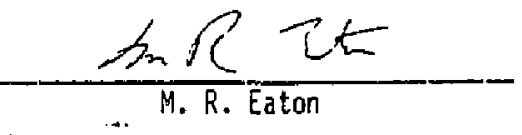

TITLE Deputy Contracts Manager

Lawrence Livermore National Laboratory DATE

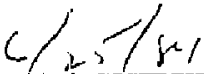


SECOND MODIFICATION TO SCHEDULE OF

SUBCONTRACT 4368005

between

THE REGENTS OF THE UNIVERSITY OF CALIFORNIA

and

BROBECK CORPORATION

INTRODUCTION

This Second Modification to Schedule of Subcontract 4368005 between the University and the Subcontractor amends, by mutual agreement of the parties, the Schedule in the following particulars only:

ARTICLE III - ESTIMATES AND ETXED FEE - delete paragraph A only and substitute therefor:

"The presently estimated cost of the work under this Subcontract is ONE HUNDRED SIXTY-SEVEN THOUSAND, EIGHT HUNDRED NINETY-SIX AND NO/100 DOLLARS $(\$ 167,896.00)$ exclusive of the Subcontractor's fixed fee. The Subcontractor's fixed fee is SIXTEEN THOUSAND, SEVEN HUNDRED EIGHTY-TWO AND NO/100 DOLLARS $(\$ 16,782.00)$. The aggregate of presently estimated cost and fixed fee is ONE HUNORED EIGHTY-FOUR THOUSAND, SIX HUWDRED SEVENTY-EIGHT AND NO/100 DOLLARS $(\$ 184,678.00) . "$

All other provisions of the Schedule of Subcontract 4368005 shall remain in full force and effect, except as amended herein.

$$
\text { (Increase } \$ 39,774.00)
$$

2nd Modification to Schedule 
SECOND MODIFICATION TO EXHIBIT I OF

SUBCONTRACT 4368005

between

THE REGENTS OF THE UNIVERSITY OF CALJFORNIA

and

BROBECK CORPORATION

INTRODUCTION

This Second Modification to Exhibit I - Scope of Hork of Subcontract 4368005 between the University and the Subcontractor amends, by mutual agreement of the parties, Exhibit I in the following particulars only:

SECTION III - TECHNICAL SCOPE OF WORK is modified, in part, in the following particulars only:

"Task II. C - Engineering Design and Detail for an Electron Beam Window

The objective of this task focuses on the Vacuum-to-Air Rotating Window design study which concentrates upon the mechanical and thermal problems of the spinning disk and its support system. Critical design items: gyrodynamics, balance and stress.

The Subcontractor's efforts shall be directed toward engineering design and detail drawings for fabrication of a prototype of the Vacuum-to-Air Rotating Window.

In conduct of this task, the stresses due to high centrifugal force, thermal shock and vacuum loading shall be part of the design consideration. University acceptable combinations of material properties, dimension and configurations shall be selected by Subcontractor for design. Subcontractor shall calculate bearing friction, aerodynamic drag and turbo-molecular pumping loads to determine drive power and parasitic heat loads. Means for radiation and convection cooling of the rotating disk shall be evaluated.

Upon completion of the engineering design for the rotating elements, Subcontractor shall complete the detail design for the Rotating Window and provide a set of drawings suitable for shop fabrication as the final documentation of this effort, acceptable to the University's Technical Representative.

SECTION IV - LEVEL-OF-EFFORT - delete as writter: and substitute therefor: 
"The distribution and allocation of technical and professional effort under this Subcontract shall be approximately as follows:

Type of Personnel

Man Hours (Estimated)

Task I

Task II

Chemistry Support ATA Support

Engineer 16

Engineering Associate ?

767

1049

$1160^{n}$

All other provisions of Exhibit I of Subcontract 4368005 shall remain in full force and effect, except as amended herein. 


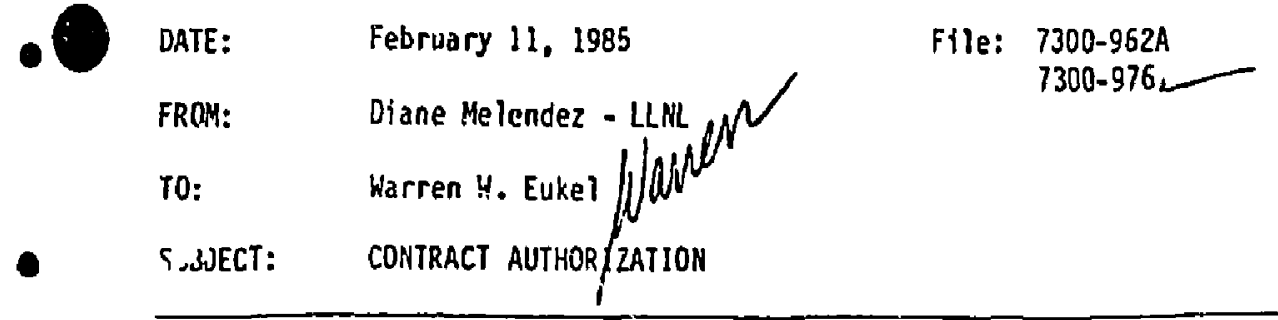

Ms. Helendez gave verbal authorization to proceed with the long lead item racurement on a CPFF Contract \$6551805. The total amount authorized is $\$ 83,997$. This is from our proposal 7300-962A. Our consolidated Proposal $\$ 7300-976$ for $\$ 240,262$ has not been approved becausa purchasing does not have a requisition for the proposal.

WWE : jW

cc: K.M. Thomas 


\section{SUBCONTRACT 6551805}

between

THE REGENTS OF THE UNIVERSITY OF CALIFORNIA

and

BROBECK CORPORATION

INTRODUCTION

This cost Plus Fixed Fee Subcontract is made and entered into by and between The Regents of the University of California, hereinafter called the "University" and Brobeck Corporation hereinafter called the "Subcontractor".

The Regents of the University of California, a California corporation, have entered into a contract, $W-7405-E N G-48$ with the United States Governntent, hereinafter called "Government", represented by the Department of Energy, hereinafter called "DOE", for the performance of certain energy research and development work at the University of California Lawrence Livermore National Laburatory. This Subcontract is entered into in furtherance of the performance of the work provided for in the prime contract.

In accepting this Subcontract, the Subcontractor agrees to perform the Subcontract work in accordance with the following documents which collectively constitute the Subcontract and are attached hereto and by this reference made a part hereof:

Subcontract Signature Page

Schedule consisting of 6 pages

Exhibit 1 - Statement of Work

General Provisions dated July 1983

(Revised 6/83)

ACCEPTEO:

BROEECK CORPORATION

BY:

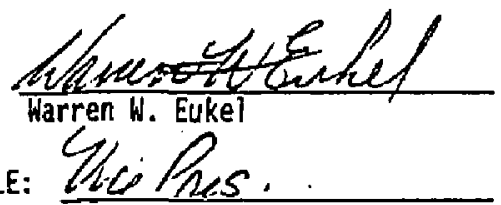

TITLE:

DATE: Elleyle, 1985

FORM RL-2365A
AUTHORIZED:

THE REGENTS OF THE UNIVERSITY OF CALIFORNIA

BY:

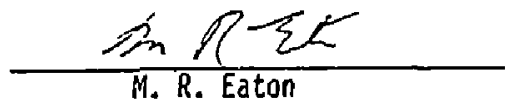

TITLE: Deputy Contracts Manager Lawrence Livermore Nationa] Laboratory DATE: $\int / x / d T$ 


\section{SCHEDULE}

\section{ARTICLE I - SCOPE OF HORK}

The Subcontractor shall furnish the necessary facilities, supplies, equipment and personnel, except such as are furnished by the University or the Government, to conduct certain work generally described as the procurement of long lead time materiais for the beam director. Subcontractor shall specify, procure, coordinate and generally provide technical guidance during the procurement of critical materials for the bean director project to be performed by the Subcontractor at iocations approved by the University and Subcontractor's facility at Berkeley, California the nature and extent of which are more specifically described in EXHI8IT I - STATEMENT OF WORK hereto, and shall also deliver such materials, products, supplies and incidental services and reports as may be set forth in said EXHIBIT 1.

The University and DOE shall have the right to inspect Government property and the work and activities of the Subcontractor under this Subcontract at such time and in such manner as they shall deem appropriate. The Subcontractor shall include in all subcontracts and purchase orders under this Subcontract involving research and development or fabrication, or where costs incurred are a factor in determining the amount payable to the vendor, a similar provision making this paragraph applicable to the subcontractor or vendor.

(Revised 6/83)

\section{ARTICLE II - TERM}

Unless completely performed prior thereto or sooner terminated in accordance with CLAUSE 14 - TERMINATION, the work described in ARTICLE I - SCOPE OF WORK, shall begin February 11,1985 and shall continue through December 31, 1985 which is the current period of time estimated as necessary for completion of said work, provided that the term of this Subcontract shall continue for such period of time as required to comply with the reporting and close out procedures of this Subcontract. Neither party guarantees the aforestated estimate of time and work under this Subcontract and, except as necessary to comply with reporting and close out procedures, the term shall cease on the last date mentioned uniess the University by written notice to Subcontractor extends, at its sole option, such period for such time or times as it considers appropriate for completion of the work described in ARTICLE I SCOPE OF WORK. Such an extension of time will not be a basis for a claim of additional fee by Subcontractor.

(Revised 2/83) 
A. Initial Estimate of Cost and Fixed Fee

The presently estimated cost of the work under this Subcontiact is SEVENTY-SIX THOUSAND THREE HUNDRED SIXTY-ONE AND OO/100 DOLLARS $(\$ 76,361.00)$ exclusive of the Subcontractior's fixed fee. The Subcontractor's fixed fee is SEVEN THOUSAND SIX HUNDRED THIRTY-SIX ANO $00 / 100$ DOLLARS $(\$ 7,636.00)$. The aggregate of presently estimated cost and fixed fee is EIGHTY-THREE THOUSAND NINE HUNORED NINETY-SEVEN AND N0/100 DOLLARS $(\$ 83,997.00)$.

B. Revised Estimate of Cost

The presently estimated cost of the work under this Subcontract may be increased or decreased by written agreement of the parties.

c. Limit on Total Amount of Al lowable Costs

Payments on account of costs shall not in the aggregate at any time exceed the amount of estimated costs specified in the Subcontract.

D. Notice of Costs Approaching Subcontract Amount - Subcontractor Excused Pending Increase.

Whenever the Subcontractor has reason to believe that the total costs of the work under this Subcontract, exclusive of fixed fee, will exceed the estimated cost of the work then in effect, the subcontractor shall promptly notify the University in writing. The Subcontractor shall also notify the University in writing when the aggregate of expenditures and cutstanding commitments allowable under this subcontract, excluding fixed fee, is equal to $75 \%$ (or such other percentage as the University may from time to time establish by notice to the subcontractor) of the estimated cost of the work then in effect. When such expenditures and outstanding commitments, excluding fixed fee, equal $100 \%$ of such amount, the Subcontractor shall make no further commitments or expenditures (except to meet existing commitments) and shall be excused from further performance of the work unTess and until the University shall increase the estimated cost of the work in accordance with Paragraph B. above.

E. The University's Right to Terminate not Affected

The giving of any notice by either party under this ARTICLE III shall not be construed to waive or impair any right of the University to terminate the Subcontract under the provisions of CLAUSE 14 TERMINATION.

F. Cost Information

The Subcontractor shall maintain current cost information adequate to reflect the cost of performing the work under this subcontract at all times while the work is in progress and shall prepare and furnish to the Un?versity such written estimates of cost and information in support thereof as the University may request. 
G. Correctness of Estimates not Guaranteed

Neither the University nor the Subcontractor guarantees the correctness of any estimate of cost or estimate of time for the performance of the work under this Subcontract, and there shall be no adjustment in the amount of the Subconiractor's fixed fee by reason of errors in the computation of estimates or differences between such estimates and the actual cost or time for the performance of the work.

(Revised 2/83)

\section{ARTICLE IV - CONSIOERATION}

Payment for allowable costs, reimbursable in accordance with ARTICLE $V$ REIMBURSEMENT OF COSTS, and of the fixed fee set forth in ARTICLE III ESTIMATES AND FIXED FEE, shall constitute complete compensation for Subcontractor's services, including profit and all items or kinds of expenses not allowable under the terms of this Subcontract.

(Revised 2/83)

\section{ARTICLE $V=$ REIMBURSEMENT OF COSTS}

A. The allowable cost of performing the work under this Subcontract shall be the costs and expenses (less applicable income and other credits) that are actually incurrid by the Subcontracior, are applicable and properly chargeable, either as directly incident or as allocable through appropriate distribution or apportionment, to the performance of the Subcontract in accordance with its terms and are determined to be allowable pursuant to this paragraph. The determination of the allowability of cost hereunder shall be based on: 1) reasonableness, including the exercise of prudent business judgment; 2) consistent application of generally accepted accounting principles, and 3) recognition of all exclusions and limitations set forth in this ARTICLE $V$, or elsewhere in this Subcontract as to types or arnounts of items of cost. This Subcontract recognizes that audit hereunder will be in accordance with this subcontract and cost principles and procedures of Subpart 1-15.2 of the Federal Procurement Regulations (4) CFR 1-15.2) as supplemented or modified by OOE PR 9-15.2 (4I CFR 9-15.2) including DOE PR 9-15.205.3 "Bidding Costs" and 9-15.205-35 "Independent Research and Development (IK\&D) and Bid and Proposal (B\&P) Costs", and as supplemented or modified by DOE PR 9-15.50 (4) CFR 9-15.50), in effect on the date of this subcontract. The audit might be performed by the Government Agency having the preponderant workload. However, DOE reserves the right to conduct its own audit of expenditures under this Subcontract.

\{Revised 9/84) 
B. OVERHEAD

1. Pending final audit and determination of overhead, the Subcontractor shall be piid a provisional overhead rate, as approved by the University from tin: to time and authorized in writing by the University. Such provisional payments will be adjusted to the actual overhead costs and expenses determined as set forth in this ARTICLE $V$ and principles herein established.

2. When actual reimbursable overhead costs have been determined pursuant to this Subcontract, aojustment shall be made for the difference between the sum to be determined and the sumi of the provisional payments made under Paragraph 1., above.

3. Notwithstanding Paragraphs $B .1$ and $B .2$ above, the parties hereto agree that University shall not be obiigated to pay any amount for Labor Overheac, exceeding 92.8\% of direct labor, and General and (Administrative Expenses (G\&A) exceeding $30.0 \%$ of total direct cost and Overhead, for the period cummencing February 11, 1985.

(Revised 2/83)

ARTICLE VI - RIGHTS TO PROPOSAL DATA

Except for technical data contained in pages (None) of the Subcontractor's proposal dated November 27, 1984 which are asserted by the Subcontractor as being proprietary data, it is agreed that as a cundition of the award of this Subcontrace, and notwithstanding the provisions of any notice appearing on the proposal, the University and Government shall have ihe right to use, duplicate and disclose and have others do so for any purpose whatsoever, the technical data contained in the proposal upon which this Subcontract is based.

(Revised 2/83)

\section{ARTICLE VII - GENERAL PROVISIONS}

A. The Subcontractor shall comply with the CLAUSES set forth in the General Provisions dated July 1983 as may be altered or modified hereunder and in the ARTICLE VIII - ALTERATIONS and ADDITIONS.

B. CLAUSE 31, parag:aphs A.1, A.2, Authorization and Consent (page no. 26 and 27), shall or shall not be applicable to this Subcontract as follows:

Paragraph A.1 is $[X]$ is not [ ] applicable to this Subcontract.

Paragraph A.2 is [ ] is not [X] applicable to this Subcuntract.

C. CLAUSE 31, paragr phs B.1, B.2, Patent Indemnity (page no. 27), shall or shall not be applicable to this subcontract as follows:

Paragraph $B .1$ is $[X]$ is not [ ] applicable to this Sutcontract.

Paragraph 8.2 is [ ] is not [X] applicable to this Subcontract. 
D. CLAUSE 31, paragraphs 0.1, 0.2, Patent Rights (page no. 28 or 36), shall be applicable to this Subcontract as follows:

Paragraph D.1 PATENT RIGHTS (LONG FORM) is [ ] is not [X] applicable to this Subcontract.

Paragraph 0.2 PATENT RIGHTS (SMALL BUSINESS FIRMS AND NONPROFIT ORGANIZATIONS, MARCH 1982) is [X] is not [ ] applicable to this Subcontract.

E. CLAUSE 31, paragraph G, Rights in Technical Data, subparagraphs G (page no.43) and $H$ (page no.44) shall or shall not be applicable to this Subcontract as follows:

Subparagraph $G$ is [X] is not [ ] applicable to this Subcontract.

Subparagraph $H$ is [ ] is not [X] applicable to this subcontract.

(Revised 6/83)

ARTICLE VIII - ALTERATIONS AND ADDITIONS

The following Clauses shall be applicable to this Subcontract:

Clause 35 Cost or Pricing Data (page no. 48)

In addition, the following Articles are herby incorporated into and made a part of this Subcontract:

ARTICLE 38 - Foreign Owership, Control, of Influence Over Contractor, Form LL 2358 FOCI -2 (5/83)

ARTICLE 39 - Organizational Conflict of Interest

A. The primary purpose of this article is to aid in ensuring that the Subcontractor is not biased in the performance of the work under this Subcontract, and does not obtain any unfair competitive advantage over other parties by virtue of its performance of this subcontract. However, nothing herein shall be construed to interfere with Subcontractor's right to the normal flow of benefits from performance of this Subcontract.

B. In the event $D O E$, the University or the Subcontractor are of the opinion - that such bias or unfair competitive advantage may exist, or appear to exist, then, (1) the University or Subcontractor will notify DOE and supply information requested by DOE based on such potential conflict of interest, subject to proprietary rights of others, and (2) if OOE is of the opinion that applicable criteria in the DOE regulations, OOE or the University upon notice from DOE will promptly inform the Subcontractor. DOE, the University and the Subcontractor will then enter into good faith discussions concerning whether a conflict of interest actually exists, and if so what appropriate measures might be taken to eliminate, avoid or mitigate such conflict. 


\section{EXHIBIT 1 - STATEMENT OF WORK}

to

SUBCONTRACT 6551805

between

THE REGENTS OF THE UNIVERSITY OF CALIFORNIA

and .

BROBECK CORPORATION

I. INTRCOUCTION

As approved and directed by the University, Subcontractor shall provide technical and professional effort direcled toward the effort specified in Part III - TECHNICAL SCOPE OF WORK.

As provided in CLAUSE 3 - CHANGES, the University may direct, in writing, charges within this Scope of Work.

II. PURPOSE

The purpose of this work is to provide ter:hnical guidance and engineering support to assure material acquisition and proper fabrication of long lead time itens for the Beam llirector.

III. TECHNICAL SCOPE OF HORK

As approved or directed by the University, Subcontractor shall furnish all necessary personnel, materials and facilities, except as may be provided for elsewhere herein, to conduct the Task described below:

\section{TASK I - Beam Director Long Lead Muterial}

jubcontractor shall initiate the procurement process to specify, procure, coordinate and generally provide technical guidance and engineering support during the procurement of the following material.

- ACHROMAT - Permanent Magnets (1 lot)

- Housing (Tooling \& Material) (1 each)

- VERNIER - Conductor (1 lot)

IV. LEVEL OF EFFORT

The distribution and allocation of technical and professional effort under this Subcontract shall be approximately as follows: 
V. REPORTS

Subcontractor shall prepare and submit the following reports to the University:

A. Financial Reports (Type A) - shall be submitted by the fifteenth of each month and shall include all costs incurred during the preceding month and outstanding comitments to the end of the month. In the event actual cost data is unavailable, Subcontractor's monthly financial report shall contain estimated expenditures, identified as such.

B. Monthly Progress Reports (Type B) - shall be submitted by the fifteenth of each month and may be informal letter summaries in a format approved by the University's Technical Representative. These reports should contain a description of work performed during the month reported and work planned for the succeeding month.

C. Final Report (Type C) - shall be submitted upon completion of the work and contain a comprehensive summary of all work results and conclusions. The form and content of Type $C$ Reports shall be acceptable to the University's Technical Representative. If so requested, a draft copy of the Report shall be submitted to the University's Technical Representative for approval prior to final typing.

D. Distribution of Reports

Reports shail be separately addressed and transmitted ta:

University of California

Lawrence Livermore National Laboratory

P. 0. Box 5012

Livermore, CA 94550

Attention: (Intended Recipient)

\begin{tabular}{|c|c|c|}
\hline Type of Report & No. of Copies & Recipient \\
\hline $\begin{array}{l}A \text { and } B \\
A \text { and } B\end{array}$ & $\begin{array}{l}1 \\
\text { As Requested }\end{array}$ & $\begin{array}{l}\text { Diane Melendez, } L-650 \\
\text { Dave Milani, L- } 544 \\
\text { Coleman Johnson, L-544 }\end{array}$ \\
\hline C & As Requested & Diane Melender, $L-650$ \\
\hline
\end{tabular}

Subcontractor shall not distribute reports of work under this Subcontract to any individual or organization other than those indicated above or an authorized representative of the U. S. Department of Energy without prior writien approval of the Contract Administrator. 


\section{E. Interim Reports}

It is understood that there will be other information exchanged between the parties from time to time. These data may be exchanged directly between the parties concerned; formal reporting and distribution is not required in these cases.

VI. COORDINATION AND ADMINISTRATION

The University's Technical Representative(s) under this Subcontract are Dave Milani and Coleman Jahnson, or their designee(s), who shall represent the University in matters relating to the technical performance of the Scope of Work described herein. During the established term of this Subcontract, the Technical Representatives will interpret the technical requirements of the Scope of Work and will determine the emphasis and direction of the Subcontractor in the conduct of the work witinin the level and allacation of effort established herein. All other matters relating to the performance of this Subcontract are reserved to the Contract Administrator. Provided, however, any technical direction which will affect the estimated cost or time for performance of this work shall require prior formal amendment of this Subcontract or prior written direction from the Procurement Manager, or his designee, as provided in CLALSE 3 - CHANGES of the Terms and Conditions. Technical direction resulting in work outside the general Scope of Work requires prior formal amendment of this Subcontract.

The University's Contract Administrator is M. R. Eaton, or his designee. All matters relating to the interpretation and administration of this Subcontract shall be conducted through the Contract Administrator. Subcontractor will direct all notices and requests for approval to the Contract Administrator; and any notice or approval from the University to the Subcontractor will be issued by the Contract Administrator.

VII. ASSIGNAENT OF PERSONMEL

It is understood and agreed that Subcontractor's key technical personnel assigned to this work shall not be reassigned or replaced without prior University approval, except where such circumstances are beyond the reasonable control of the Subcontractor.

VIII. HEALTH, SAFETY AND FIRE PROTECTION

The Subcontractor shall take all reasonable precautions in the performance of the work under this Subcentract to protect the health and safety of enployees and members of the public and to minimize danger from all hazards to life and property, and shall comply with all pertinent health, safety, and fire protection regulations and requirements (including reporting requirements) of the University and DOE communicated to the Subcontractor. 
In the event the Subcontractor fails to comply with said regulations or requirements of the University or the $O O E$, the University may without prejudice to any other legal or contractual rights of tha University, issue an order stopping all or any part of the work; thereafter, a start order for tine resumption of work may be issued at the discretion of the University. 
FIRST AMENOMENT

to

SUBCONTRACT 6551805

between

THE REGENTS OF THE UNIVERSITY OF CALIFORNIA

and

SROBECK COROPORATION

INTRODUCTION

This First Amerdment to Subcontract 6551805 is entered into by and between The Regents of the University of California, hereinafter called "University", and Brobeck Corporation, hereinafter called "Subcontractor".

\section{PURPOSE}

The purpose of this First Amendment to Subcontract 6551805 is to incorporate the following changed documents into this Subcontract.

NOW, THEREFORE, by mutual agreement of the parties, the following Subcontract documents, attached hereto are made a part of this Subcontract:

\section{Modification to SCHEOULE}

Modification to Exhibit 1 - STATEMENT OF HORK

All other terms, conditions and provisions of Subcontract 6551805 shall remain in full forc? and effect, except as amended herein.

IN WITNESS WHEREOF, effective upon Subcontractor's signature acceptance of this First Amendment, Subcontract 6551805 is hereby amended by mutual Agreement of the parties.

ACCEPTED:

BROBECK CORPORTION
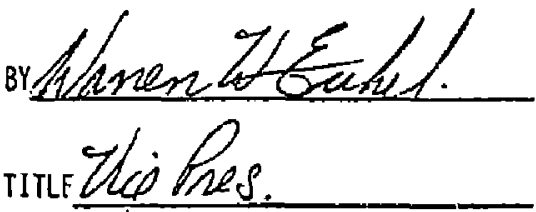

DATE $2 / 20 \% / 8 \%$
AUTHORIZED:

THE REGENTS OF THE

LNIVERSITY OF CALIFORNIA

BY

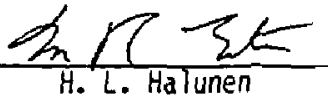

TITLE Contracts Manager Lawrence Livermore National Laboratory DATE

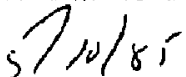




\section{FIRST MODIFICATION TO SCHEDULE}

$$
\text { of }
$$

SUBCONTRACT 6551805

\section{between}

THE REGENTS OF THE UNIVERSITY OF CALIFORNIA

and

BROAECK CORPORATION

\section{INTRODUCTION}

This First Modification to SCHEDULE of Subcontract 6551805 between the University and the Subcontractor amends, by mutual agreement of the parties, the SCHEDULE in the following particulars only:

ARTICLE III - ESTIMATES AND FIXED FEE, delete Paragraph $A$. only and substitute therefor:

"The presently eatimated cost of thc york under this Subcontract is Tw0 HUNORED EIGHTEEN THOUSAND FOUR HUNDRED TWENTY DOLLARS AND NO/TOO $(\$ 218,420.00)$ exclusive of the Subcontractor's fixed fee. The Subcontrator's fixed fee is TWENTY THOUSAND SEVEN HUNDREO FIFTY DOLLARS AND NO/100 $(\$ 20,750.00)$. The aggregate of present?y estimated cost and fixed fee is TWO HUNDRED THIRTY-NIME THOUSAND ONE HUNDRED SEVENTY DC.LARS AND NO/100 $\left(\$ 239_{2}, ? 70.00\right) "$

A11 other provisions of the SCHEDULE of Subcontract 6551805 shall resain in full force and effect, except as amended herein. 


\title{
FIRST MODIFICATION TO EXHIBIT I
}

to

\author{
SUBCONIRACT 6551805
}

between

THE REGENTS OF THE UNIVERSITY OF CALIFORNIA

and

BROBECK CORPORATION

\section{INTRODUCTION}

This First Modification to EXHIBIT 1 - STATEMENT OF Y.JRK to Subcontract 6551805 between the University and the Subcontractor amends, by mutual agreement of the parties, EXHIBIT 1 in the following particulars on?y:

SECTION III - TECHNICAL SCOPE OF WORK is mocified, in part, in the following particulars only:

Add TASK II:

"TASK II - Engineering:Support, Fabrication, Assembly and Bench Test Vernier and Achromatic Magnet As sembly

Subcontractor shall fabricate, assemble, bench test, and deliver to LLNL the Achramat Magnet Assembly and the Vernier Steering Magnet Assembly. In conduct of this work, Subcontractor's work shall include, but is not limited to the following:

Procurement of materials, fabrication, selection, and supervision of specialty sub-subcontractors."

SECTION IV - LEVEL OF EFFORT, is hereby amended to add the following estimate of effort:

"Type of Personne]

Engineer Senjor

Engineer Designer

Engineer Associated 7 (Tech)
Man Hours (Estimated)

274

398

1314"

Add SECTION IX - Materials, to this Subcontract.

"SECTIOH IX - Materials

Subcontractor shall deliver all assemblies to LLNL FOB Destination."

All other provisions of EXHIBIT 1 of Subcontract $\$ 551805$ shall remain in full force and effect, except as amended herein.

Ist Mod. to Exhibit 1 
APPENDIX B. REQUESTS FOR QUOTATIONS (RFQ'S)

\begin{tabular}{|c|c|c|c|c|}
\hline RFQ & DATE & $\operatorname{PART}(S)$ & P.0. & AKARDED TO \\
\hline $4500-300-A$ & $2-12-85$ & Magnet segments & 086 & Permag Sierra Corp. \\
\hline $4500-300-B$ & $2-20-85$ & Hous ing & 110 & Edwards Enterprises \\
\hline $4500-300-1$ & $4-23-85$ & Segment carriers & 122 & Bennett-Hopkins \\
\hline $4500-300-2$ & $5-1-85$ & Magnet spacers, etc. & 128 & Accurate $M f g$. \\
\hline $4500-301-3$ & $5-17-85$ & Spool, coil spacers, etc & c 143 & Oi amond Tool \\
\hline $4500-300-C$ & $10-1-85$ & Spare segment carriers & 239 & Diamond Tool \\
\hline $4500-301-6$ & $10-4-85$ & Vacuum housing parts & $\begin{array}{l}1253 \\
1254\end{array}$ & $\begin{array}{l}\text { (Caral, Inc. } \\
\text { (Schrader scientific }\end{array}$ \\
\hline $4500-300-7$ & $10-9-85$ & Vacuum vessel parts & 255 & $\begin{array}{l}\text { Jensen Prototype } \\
\text { Machines }\end{array}$ \\
\hline
\end{tabular}


February 12, 1985

File: $4500-300$

[RFQ 4500-300-A]

Ms. Gall Fernandez
Permag Sterra Corporation
1159 Sonora Court
Sunnyvale, Caltfornta 94086

Dear Ms. Fernandez:

You will recall your Quotation No. 2761 of 9-4-84 for dipole and quadrupole magnets. You had been contacted by Mr. Francis C. Younger of our firm. Since then, there have been some minor changes which necessitate an updated quotation.

We are requesting a quotation on permanent magnets shown dimensionally on our drawings:

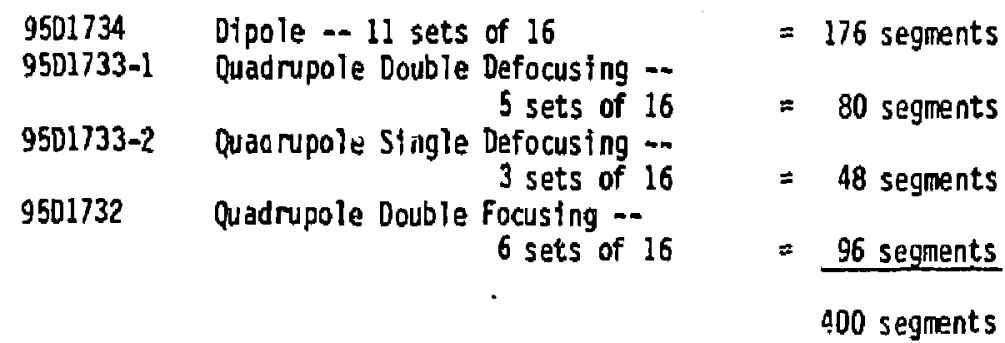

Please note that some of the quadrupoles are long and some are short. This is described on the enciosed drawings.

The magnets are to be made of a material having the magnetic properties of Cructble Magnetics "Ferrimag .78." A residual induction of 3800 Gauss and a coersive force of 3500 Dersteds are required. The residual induction and coercive force shall not vary by more than $2 \%$ from magnet to magnet and the direction of magnetization shall be as shown on the drawings plus or minus 2 degrees. This requires that all the magnet segments shall be of the same betch and shall be so certiftpd.

Please note that the ends of the dipole segments are not orthogonal to the sides whereas the ends of the quadrupole segments are orthogonal. This will undoubtedly require special care in machining operations.

The magnetic orientation of each finished segment nust be clearly shown to facilitate proper assembly in the magnet housings. We prefer arrows showing North as is done in the drawings. Each segment shall be marked with the proper drawing number so as to assist the assembly operation. 
Please quote promptly both as to price and delivery since we are anxious to fabricate the magnet assemblies of which these are a part.

If you have any questions, please call Mr. Younger at 524-8664.

\section{JTG:jW}

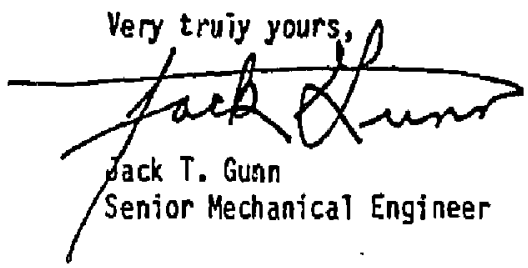

Enclosures. 
FOR FILE: See attached mailing list.

[AOR]

Subject: REQUEST FOR QUOTATION 4500-300-B

ACHROMAT HOUSING

Dear [MAME]

We request your quotation on furnishing one (1) Achromat housing per Brobeck Corporat ton Drawing Number 951 1739.

Because of budget constraints, the work is to be performed in two stages as outlined below. Each stage should be separately priced and quoted as to required delivery time af ter your receipt of out" order to proceed with each phase.

Phase One - Tooling and stock procurement.

a. Purchase aluminum stock.

b. Machine parting line surfaces.

c. Temporarily join the two halves.

d. Machine outside diameter to finish size.

e. Manufacture all jigs necessary for Phase Iwo.

Phase Two - Fabrication.

d. Perform all work nezessary to produce the finished part per Drawing No. 95J1739.

Prices should be based on delivery to Brobeck Corporation fn Berkeley, CA.

We would appreciate any constructive suggestions you care to make that will, in your opinion, reduce the cost without impairing function. 
Your attention is particularly directed to Note 3 of Orawing $\$ 511739$. This may require extra care in the machining. and annealing sequence. Please have your quotation in' iomation to Brobeck Corporation by March 15, 1985.

\section{JTG:jw}

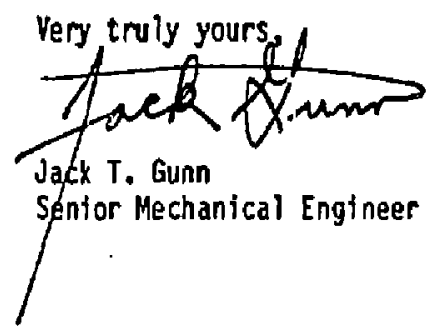

Enclosure: Dwg. No. 9511739. 
RFQ 4500-300-B sent to:

[ADR] 1;

Allied Engineering Company

$2421 \mathrm{Blanding} \mathrm{Street}$

Al ameda, CA 94501

[ADR] 2;

Bennett-Hopkins Company

3592 Haven

P.0. Box 53076

Redwood City, CA 94063

[ADR] 3;

Caral Inc.

578 Cleveland Avenue

Al bany, CA 94706

[ADR] 4;

Edwards Enterprises

8455 Cabot Court

Newark, CA 94560

[ADR] 5;

Numeric Machine Company

4439 Enterprise

Fremont, CA 94538 
April 23, 1985

File: $4500-300$

FOR FILE: See attached mailing list.

[ADR]

Subject: REQUEST FOR QUOTATION 4500-300-1

SEGMENT CARRIERS

Dear [NAME]

We request your quotation on the fabrication and delivery of the following it ems.

Item 1. 8 each Segment Carrier - Dipole \#95C1737

Iten 2. 7 each Segment Carrier : Quadrupole, long $\$ 95 \mathrm{C} 1736-1$

Item 3. 2 each Segment Carrier - Quadrupole, short $\$ 9501736-2$

DELIVERY: Two of Item 1, two of Item 2, and two of Item 3 within 6 weeks ARO.

Balance, 10 weeks ARO.

Please quote according to above.

Since these carriers are for the support of critical elements in a physics apparatus, it is important that the form tolerances be met. If you have any reservations about meeting these requirements, please so indicate on your bid.

We request your written quotation within ten (10) days of receipt of this letter.

JTG:jW

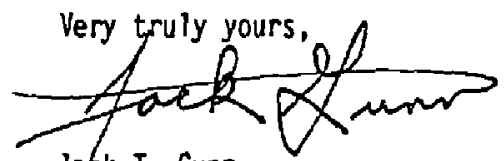

Jatk T. Gunn

Senior Engineer 
RFQ 4500-300-1 sent to:

[AOR] 1;

Allied Engineering Company

2421 Blanding Street

Alameda, CA 94501

[ADR] 2;

Bennett-Hopkins Company

3592 Haven

P.0. Box 53076

Redwood City, CA 94063

[AOR] 3;

Caral Inc.

578 Cleveland Avenue

Albany, CA 94706

[ADR] 4;

Coast Metal Cutting Company

2500 Bay Road

Redwood City, CA 94063

[ADR] 5;

Diamond Tool \& Die Company

508 - 29th Avenue

0akland, CA 94601

[ADR] 6;

Edwards Enterprises

8455 Cabot Court

Newark, CA 94560

[ADR] 7;

Numeric Machine Company

4439 Enterprise

Fremont, CA 94538 
May 1, 1985

File: $4500-300$

FOR FILE: See attached mailing list.

[ADR]

Subject: REQUEST FOR QUOTATION 4500-300-2

MAGNET SPACERS + OTHER PARTS

Dear [NAME]

We solicit your bid on the following items:

\begin{tabular}{clc} 
Quantity & \multicolumn{1}{c}{ Name } & Drawing No. \\
18 & Magnet Spacer & $95 \mathrm{~B} 1738$ \\
1 & Flange Ring & $95 \mathrm{C} 1744$ \\
1 & Adjusting Sleeve & $95 \mathrm{C} 1745$ \\
1 & Retaining Flange & $95 \mathrm{~B} 1746$ \\
1 & Retaining Sleeve & $95 \mathrm{~B} 1747$ \\
2 & Barrier Ring & $95 \mathrm{~B} 1748$
\end{tabular}

Please quote based on delivery via UPS to Brobeck Corporation in Berkeley. Clase of bidding is May 15, 1985.

Please feel free to telephone me to discuss any questions you may have regarding these parts.

JTG:jw

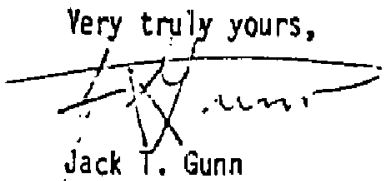

Enclosures: Drawings.

(415) $524-8664$ 
RFQ 4500-300-2 sent to:

\section{[1]}

Accurate Manufacturing Company

4770 San Pablo Avenue

Emeryville, CA 94608

\section{[2]}

August Manufacturing Company

1466 36th Avenue

Oakland, CA 94601

[3]

Caral Inc.

578 Cleveland Avenue

Albany, CA 94706

\section{[4]}

- International Precision Machining 23839 Connecticut Street, Unit $\# 5$ Hayward, CA 94545

\section{[5]}

McNeill Manufacturing Company

2914 E. Seventh St.

Oakland, CA 94601 
May 17, 1985

File: $4500-301$

FOR fILE: See attached mailing list.

[ADR]

Subject: REQUEST FOR QUOTATION 4500-301-3

SPOOL, COIL SPACERS, AND OTHER PARTS

Dear [NAME]

We solicit your quotation on the following parts (drawings enclosed):

GROUP A

\begin{tabular}{lll} 
Number & Name & Qty \\
\hline $95-\$ 1707$ & Spool & 1 \\
$95 C 1708$ & Locknut & 1 \\
$95 B 1717-1$ & Clamp & 4 \\
$9581717-2$ & Clamp & 4 \\
9501723 & Power rlange & 1 \\
$95 C 1724$ & Cooling Flange & 1 \\
9581727 & Spreader & 1 \\
$95 B 1729$ & Terminal & 1
\end{tabular}

Since somewhat different machining capabilities are involved, we would appreciais a separate quote on:

\section{GROUP B}

$\begin{array}{lll}95 C 1712 & Y \text { Coil Spacer } & 1 \\ 9501713 & X \text { Coil Spacer } & 1\end{array}$

Close of bidding is May 31, 1985. Please quote separately on either, or both, of Groups $A$ and $B$. State terms and conditions and include delivery to Brobeck Corporation via UPS.

I am available to clarify any aspect of the drawings. We would appreciate your comments and suggestions as to how to improve the cost or the desion of these parts. Please feel free to phone.

WhE: jW

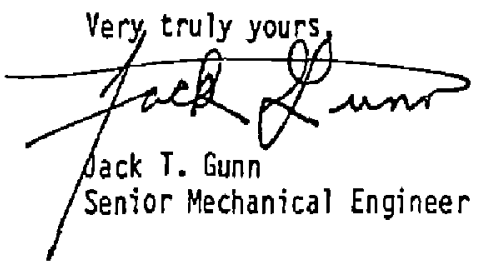

Enclosures: Drawings.

(415) 524-8664 
RFQ 4500-301-3 sent to:

[1]

Allied Engineering Company

2421 Blanding Street

Alameda, CA 94501

[2]

August Manufacturinọ Comp̣any

1466 36th Avenue

0akland, CA 94601

[3]

Caral Inc.

$578 \mathrm{Cleveland}$ Avenue

Albany, CA 94706

[4]

Di amond Tool \& Die Company

508 - 29th Avenue

Oakland, CA 94601

[5]

Edwards Enterprises

8455 Cabot Court

Newark, CA 94560 


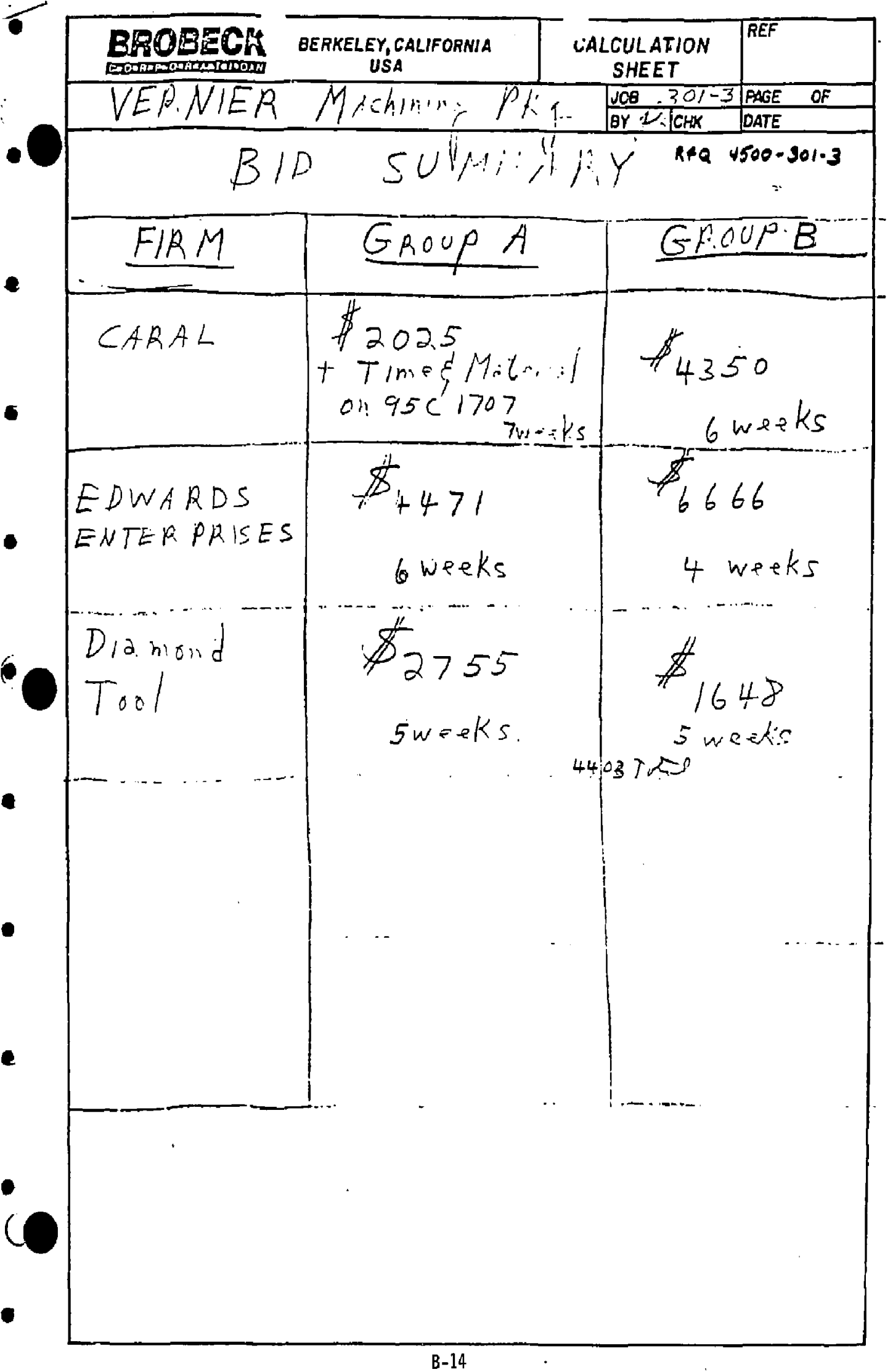


FOR FILE: See attached malling list.

[ADR]

Subject: REQUEST FOR QUOTATION 4500-301-6

VACUUM HOUSING PARTS

Dear [NARE]

We solicit your bid on the listed vacuum housing parts. All parts are to be certified leak free when tested with a helium nass-spectrometer leak detector and must be scrupulously clean for high vacuum service. All sealling surfaces must be protected so as to be free of scratches and nicks.

CLOSE OF BIDS: October 14, 1985.

SCHEDULE REQUIREMENTS: Parts needed $b ;$ November 6, 1985.

Please bid each item separately. He reserve the right to award bids on a piece-by-piece basis. Telephone bids are encouraged. We welcome any suggestions which wrll make these parts less expensive without compromising their performance.

\begin{tabular}{|c|c|}
\hline Drawing No. & Name \\
\hline $95 C 1772$ & Cover Plate \\
\hline $95 C 1771-1$ & Pumpout Spool \\
\hline $95 C 1771-2$ & Pumpout Spool \\
\hline $95 C 1770$ & Spool \\
\hline 9501769 & 2 Port Tank \\
\hline $95 C 1724$ & Cooling flange \\
\hline 9501773 & Port Cover \\
\hline
\end{tabular}

Drawings are enclosed for bid purposes only.

Very truly yours,

(415) 524-8664

1235 Tenth Streat 
RFQ 4500-301-6 sent to:

\section{[1]}

Accurate Manufacturing Company

4770 San Pablo Avenue

Emeryville, CA 94608

[2]

Caral Inc.

578 Cleveland Avenue

Albany, CA 94706

\section{[3]}

Di amond Tool \& Die Company

508 - 29th Avenue

Oakl and, CA 94601

\section{[4]}

MeNeill Manufacturing Company

2914 E. Seventh St.

Oakland, CA 94601

[5]

Schrader Scientific Company

2976 Arf Avenue

Hayward, California 94545

\section{[6]}

Jensen Prototype Machines

940 Lemon Street

Martinez, California 94553 

FOR FILE: See attached mailing list.

[ADR]

Subject: REQUEST FOR QUSTATION 4500-300-7

VACUUM VESSEL PARTS

Dear [NAME]

He solicit your bid on the following vacuum vessel parts. Since they will be in a high-vacuum environment, they must be scrupulously clean. Strict adherence to the drawing tolerances is required, particularly to the concentricity call outs.

CLOSE OF BIOS: October 17, 1985.

SCHELULE REQUIREMENTS: Parts needed by Movember 12, 1985.

Telephone bids are encouraged. We weicome any suggestions which will make these parts less expensive without compromisting their performance.

$\begin{array}{llll}\text { Drawing No. } & \text { Name } & \frac{\text { Oty }}{2} \\ 9581777 & & \text { Plate } & 2 \\ 95 B 1778 & \text { Spoot } & 1 \\ 95 B 1779 & \text { Bearing } & 1 \\ 9581780 & \text { Bar } & 2 \\ 95 C 1774 & \text { Flange } & 1 \\ 95 C 1773 & \text { Cover Plate } & 1 \\ 95 C i 775 & \text { Adapter Plate } & 1 \\ 95 C 1776 & \text { Bolt Ring } & 1\end{array}$

Oravings are enclosed for bid purposes only.

Very truly yours,

(415) 524-8664 
RFQ 4500-300-7 sent to:

[l]

Accurate Manufacturing Company

4770 San Pablo Avenue

Emeryrille, CA 94608

[2]

Allied Engineering Company

$2421 \mathrm{Bl}$ anding Street

A? ameda, CA 94501

[3]

Bennett-Hopkins Company

3592 Haven

P.D. Box 53076

Redwood City, CA 94063

[4]

Caral Inc.

578 Cleveland Avenue

Albany, CA 94706

[5]

Diamond Tool \& Die Cimpany

508 - 29th Avenue

Dakland, CA 94601

[6]

Jersen Piototype Machines

940 Lemon Street

Martinez, Cal ifornia 94553 
s 50

c

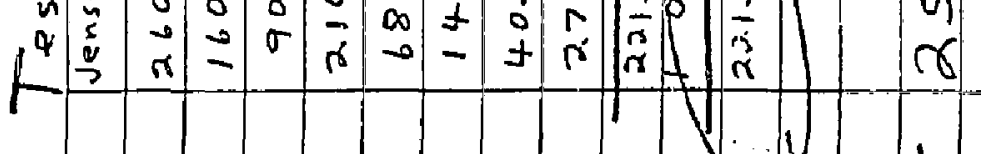

$\dot{4}$

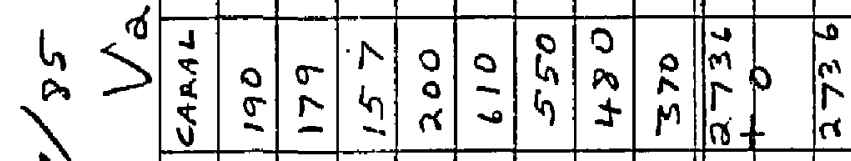

AN

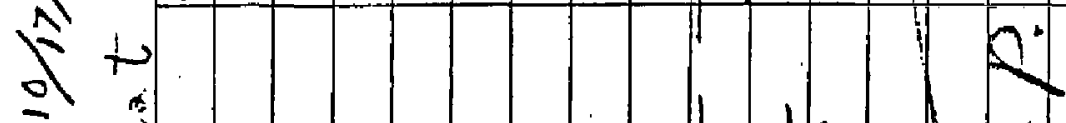

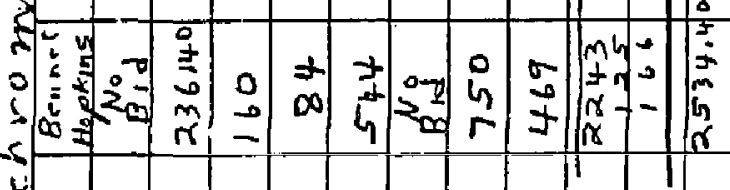

Q

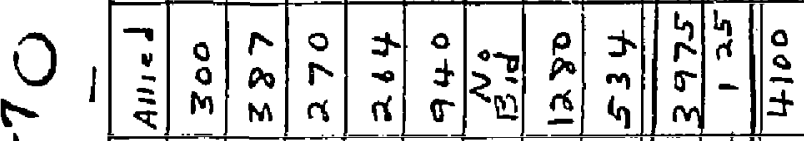

b.

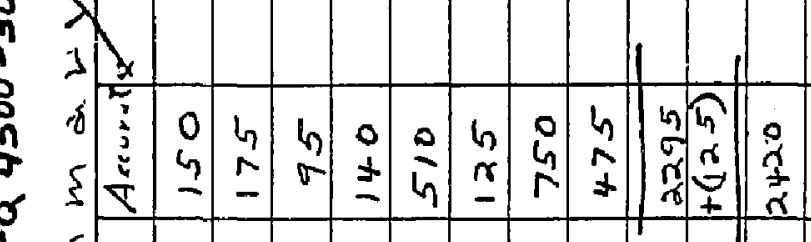

u $\$$

2

(n)

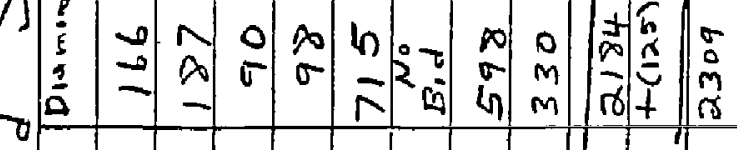

Q

G $r-r-\rightarrow-$

U

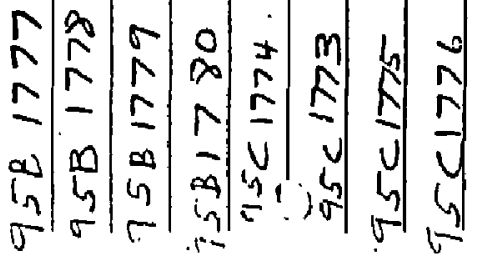




\section{APPENDIX C. PURCHASE ORDERS}

All purchase orders from the achromat and vernier design-and-build project are included here, in numerical order. The purchase orders are listed in Tables 9-1 (achromat) and 9-3 (vernier) in \$9.0, Volume 1 .

NOTE: The achromat is Job Number 300 (Acrount $4500-300$ ) and the vernier is Job Number 300 (Account 4500-301). 


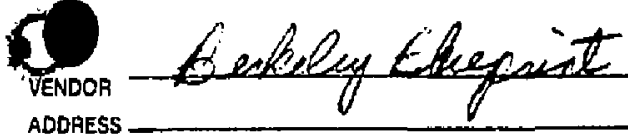

\begin{tabular}{|c|c|c|}
\hline \begin{tabular}{l|l} 
P.D & 192 \\
No & 0
\end{tabular} & $\checkmark$ & DISPOSITION \\
\hline DATE NEEDED & & TYPE P.O. L MAIL \\
\hline \multirow[t]{2}{*}{ SHIP VIA } & & ORDER WIRED \\
\hline & & OHDË TELEPHONED \\
\hline$F 08$ & & $\begin{array}{l}\text { DATE } \\
2-15-15\end{array}$ \\
\hline
\end{tabular}

MTTN. OF

DATE PROMISEO

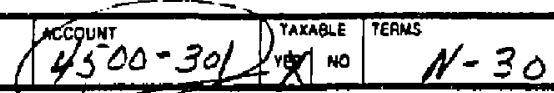

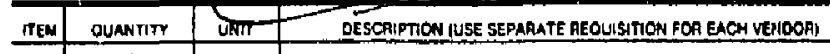

ESTIMATED ESTIMATED
IMII PAICE

\section{Fuh up o lebier}

ESTIMATED TOTAL VALUE OF' ORDER MUST BE STATED BY REQUISITIONER $\rightarrow \$$

\section{SALES TAX}

\section{Suggested Squrces}

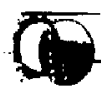

Previous Purchase Order No 
BROBECK CORPORATION COPY

II3S TENTH STREET - BERKELEY, CA 94710 - (415) 524-Bi164

\section{0}

THIE MUMERA MUST ADHLAC OM ALL INYOICE. MACKAGTI

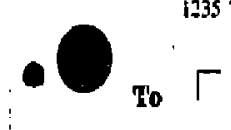

- PERMALI:eTER INC. 1844 North Keystone Burbank, CA 91504

\begin{abstract}
. BROBECK CORPORATION
1235 Tenth Street

Berkeley, CA 94710
\end{abstract}

Harry Walker (213) 849-4543

\begin{tabular}{|c|c|c|c|c|c|c|c|c|}
\hline \multirow{2}{*}{\multicolumn{3}{|c|}{$\frac{\text { Harry Walker }}{2-20-85}$}} & \multirow{2}{*}{ 213) $849-4543$} & & UPS & \multirow{2}{*}{ B.o.e. } & \multirow{2}{*}{ Tingutón } \\
\hline & & & & nesowint & 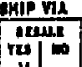 & $\lim _{0}$ & & \\
\hline - & 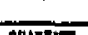 & 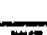 & & 7 & & & Brobeck & JIG \\
\hline
\end{tabular}

1. 2000 feet Aluminum Conductor Alloy EC-0 or 1100-0. 0.020 inch $\times 0.250$ inch cross section.

After slitting, to be carefully deburred and then insulated with eiectrical grade anodizing 0,002 inch thick. To be wound on coils reels not less than 6 inches inside dianeter. Minimum length to be 100 feet.

Transportation charge

C.0.D, charge

TOTAL

$\$ 600 / 70+\$ 600.00$

(PER TELEPHONE QUUTATION OF \$600 PER

HARRY WALKER ON 2-19-85),

RESALE NO.: SR CH 21-140517 


\section{BROBECK CORPORATION}

1235 TENTH STREET - BERKELEY. CA 94710 • (415) 524-8664

Ship to:
086

THIL mudich aut apetan ON ALL INvOVETE, racketoro AWB UMTFIMA PAFEMI.

.0

To

- PEPMAG

1159 Sonora Court

Sunnyvale, CA 94086

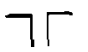

PURCHASE ORDER

BROBECK CORPORATION

1235 Tenth Street

Berkeley, CA 94710

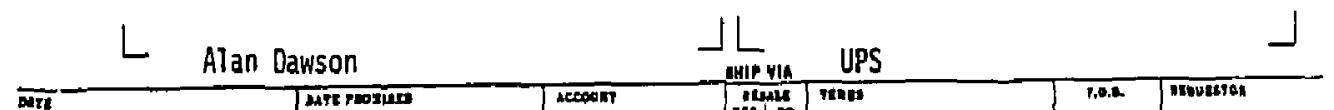

\begin{tabular}{|c|c|c|c|c|c|c|c|}
\hline 3-1-85 & & $\begin{array}{l}\text { nire rosins } \\
8-10 \text { weeks } \\
\text { Need by } 5-1-85\end{array}$ & $\begin{array}{l}45000-300 \\
4500\end{array}$ & \begin{tabular}{l|l|}
$\sin$ \\
$x$
\end{tabular} & $N-30$ & 1.0.1. & $\begin{array}{l}\text { movetsan } \\
\text { 1. Gunn }\end{array}$ \\
\hline 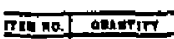 & Din & & gtachloric & & & Sinir fale? & 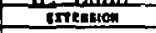 \\
\hline
\end{tabular}

1. 176 segmerts per Drawing 9501734 per Quotation \#2870, 2-22-85

$\$ 75.86 \$ 13,351.36$

2. 80

segments per Drawing 9501733-1 per Quotation \#2871, 2-22

56.86

$4,548.80$

3.

48

segments

per Drawing 9501733-2 per Quotation \$2872, 2-22

47.39

$2,274.72$

4.

96

segments

per Drawing 9501732 per Quotation $\$ 2873,2-22-85$

56,86

$5,458.56$

TOTAL

RESALE NO.: SR CH 21-240517

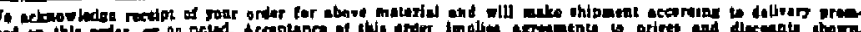

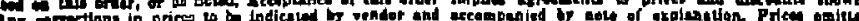
Ans Fran

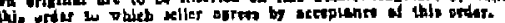

VENDOR BY C-4
1 MPORTANT

TERUS AND COFDTIUNS ON THE BACK OF TELS ORDKR ARE MADE A PABT OE TIIIS ORTER SELLFE AGRESS TO THESE CONDITONS BY ACCLPTANCE OF THE ORDER 
WIUUAM M. BROPACK \& ASSOCIATES,

RESEARCH - DEVEL, MTENT - ENGINEERING

INDUSTRY - BUSINESS - SCIENCE

1235 Teath St. Derkeley, $\mathrm{CA} 94710$ (415) 524-8664

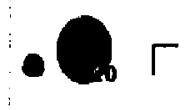

EDTAROS ENTERTIESS

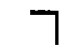

8455 CAMOT COURT

NGSARE, CALIFGEAIA $9456 \mathrm{~N}$

TH19 MUMEeA Hust APrean

on ALL I HYOICT, PACXhGt: AWO OMIPPIND PAPERE.

PURCHASE ORDER

SHIP AND ALLL TO

1235 Tenth Street

Berkeley, CA 94710

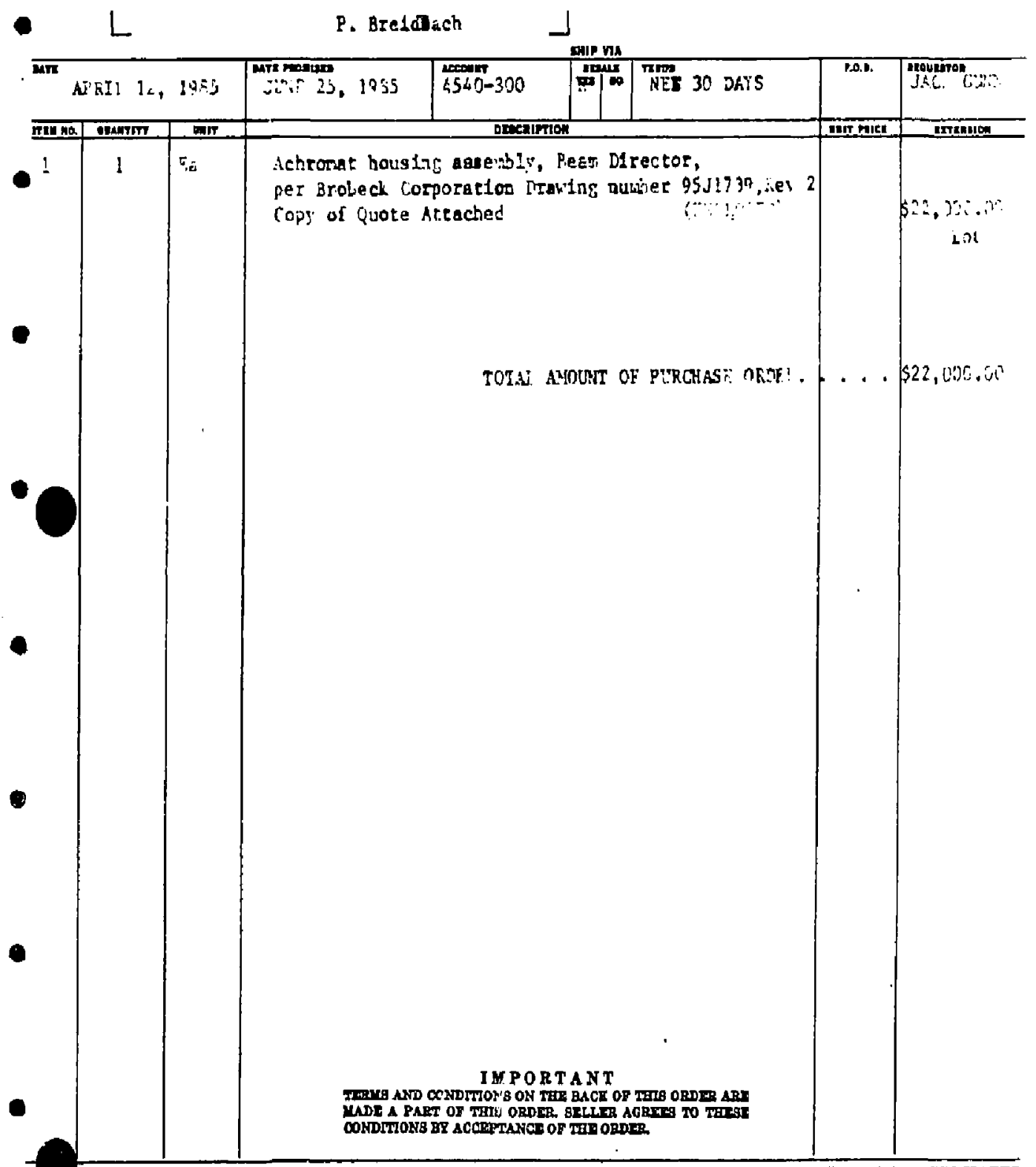

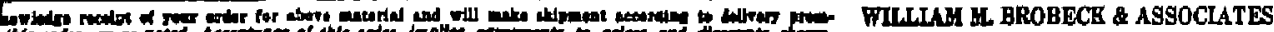

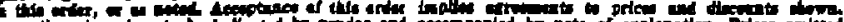

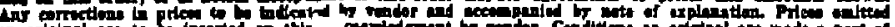

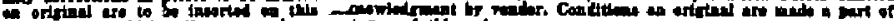

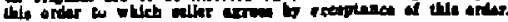


ALL ITEMS PRINTED IN RED MUST

BE FILLED IN BY REOUISITIONER

PURCHASE REQUISITION

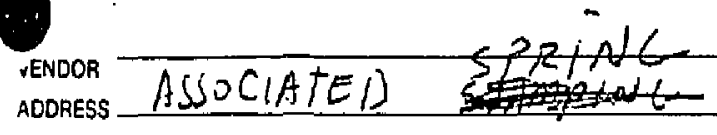

\begin{tabular}{|c|c|c|c|c|}
\hline \multicolumn{2}{|c|}{\begin{tabular}{l|l}
.0 & 129 \\
\end{tabular}} & $\checkmark$ & \multicolumn{2}{|c|}{ OISPOSITION } \\
\hline DATE NEEDE & & & \multicolumn{2}{|c|}{ TYPE P.O. MALL } \\
\hline \multirow[t]{2}{*}{ SHIP VIA } & \multirow[t]{2}{*}{ - } & & \multicolumn{2}{|c|}{ OADER WIRED } \\
\hline & & & \multicolumn{2}{|c|}{ ORDER TELEPHONEO } \\
\hline \multicolumn{3}{|l|}{ FOA } & \multicolumn{2}{|c|}{$5 / 16 \pi$} \\
\hline $\begin{array}{l}\text { ESTIAATED } \\
\text { WNIT PAILE } \\
\end{array}$ & $\begin{array}{l}\text { ESTIMATED } \\
\text { TOTA PAICE }\end{array}$ & & $\begin{array}{l}7 \text { TUNT } \\
\text { AICE }\end{array}$ & TOTAL \\
\hline & 390.0 & & & \\
\hline
\end{tabular}

$2 \quad S P R I N C$

RESALE NS.

SR $C H .21-140517$

ESTIMATED TOTAL VALUE OF ORDER MUST BE STATED BY REOUISITIONER $\rightarrow \$ 390.0 \mathrm{~J}$

DELIVEA TO 


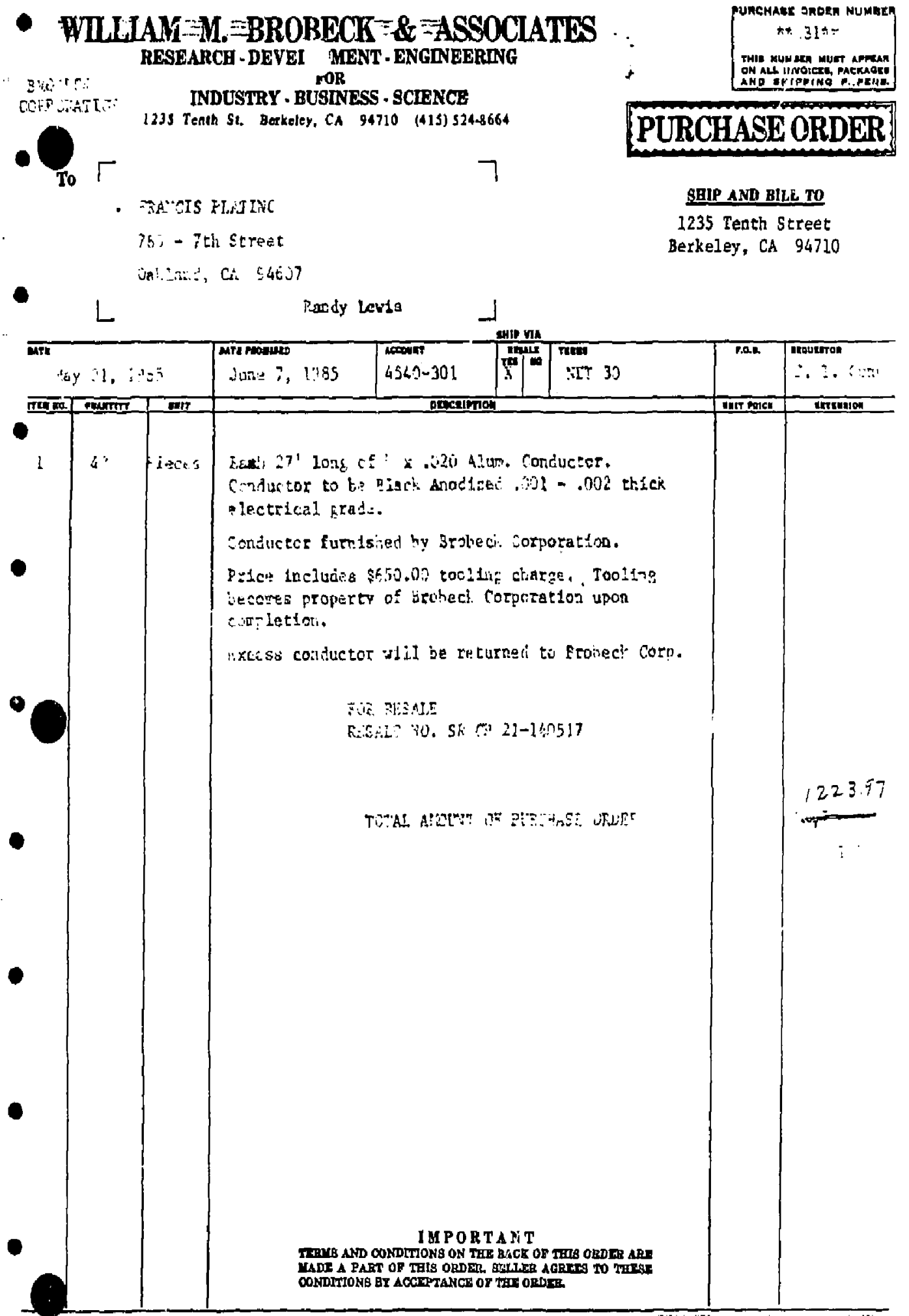

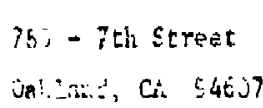

1235 Tenth Street

Berkeley, CA 94710

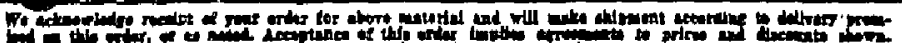

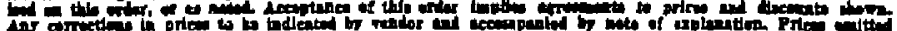

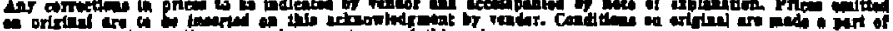

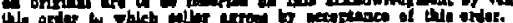

TMATA XF EROBEC

Fisi: 2.9

: 
- WILLIAM M. BRORFCK \& ASSOCIATES RESEARCH.DEVEL MENT-ENGDVERRIG roR

INDUSTRY - BUSINESS - SCIENCE

1235 Teath St Betkeity, CA 94710 (415) S24-8664

$\cdot$ $\because$.

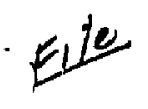

PUREHAER OHDER NUNERR în

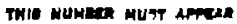

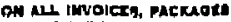
AND NAIPFING PAPERE.

PURCHASE ORDER

SBIP AND BILL TO

1235 Tenth Street Berkeley, CA 94710
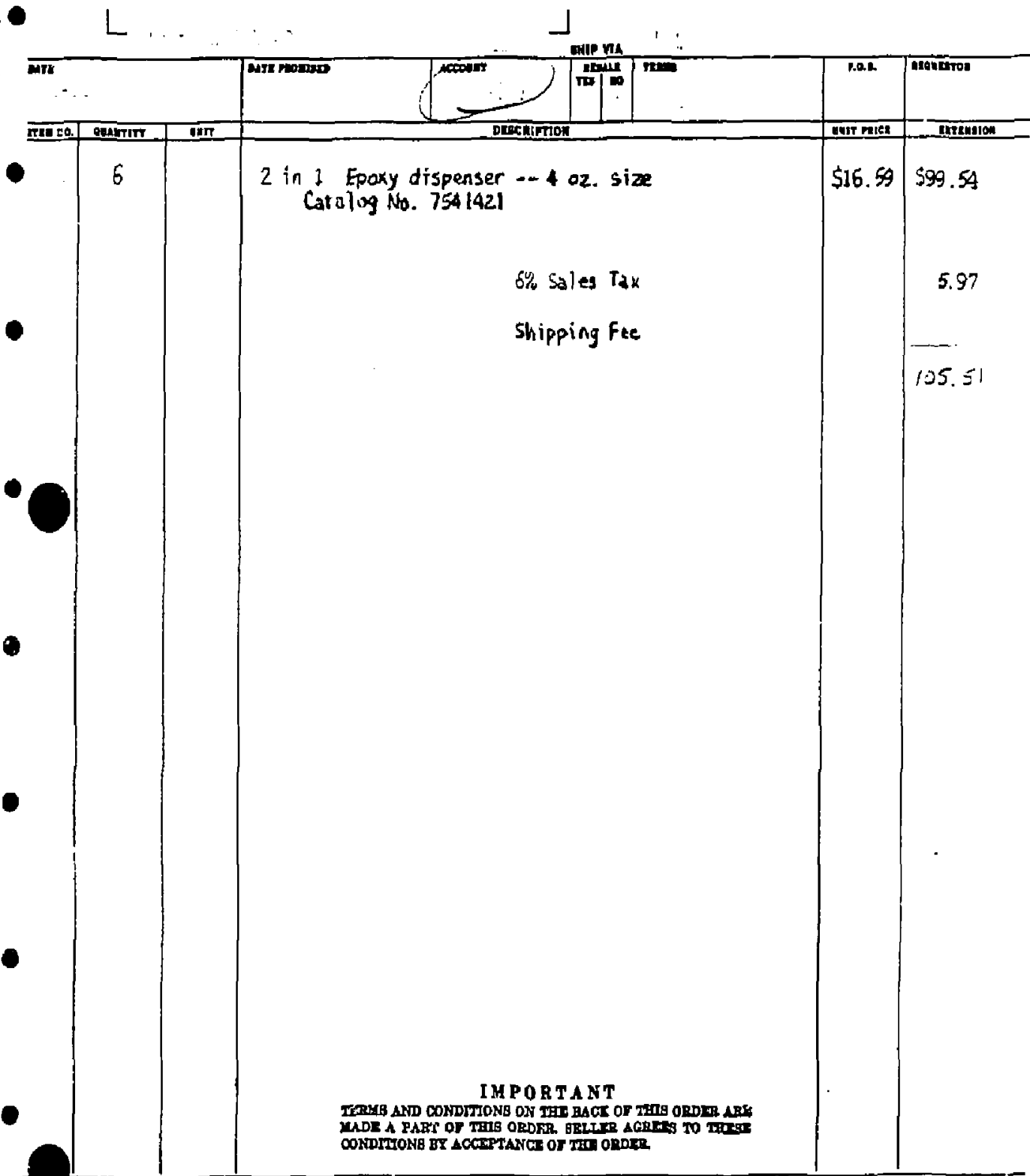
COND

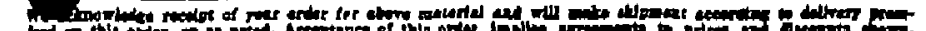

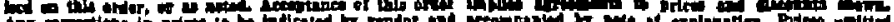

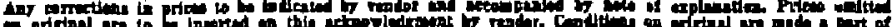

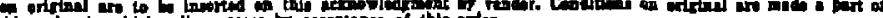

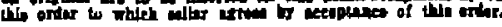


WILLIAM M. BROBECK \& ASSOCIATES

ALL ITEMS PRINTED IN RED MUST BE FILE IN BY REOUISITUNER PURCHASE REQUISITION

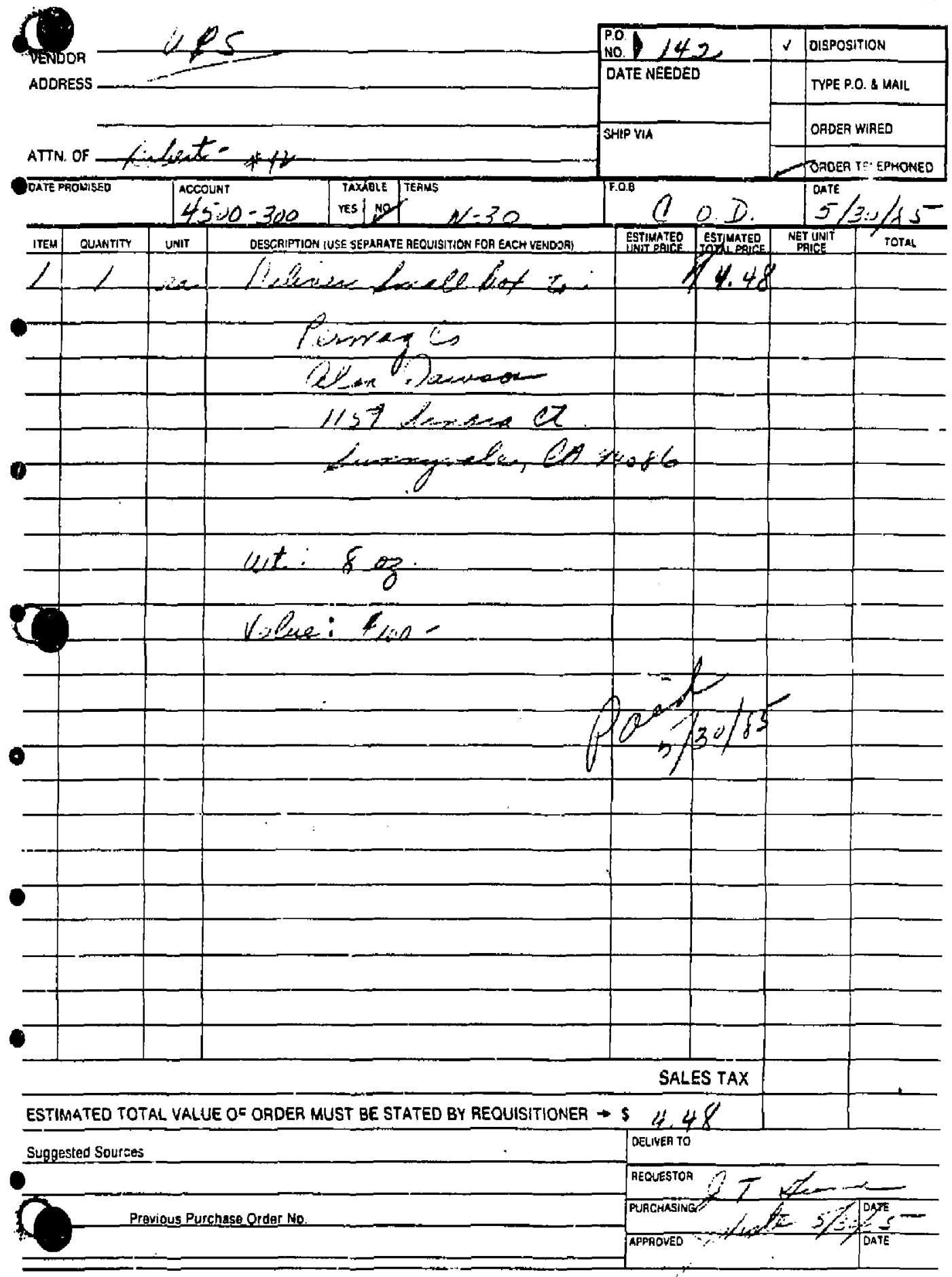

8A-30 (REV 1-J9)

ACCOUNTS PAYABLE

$C-13$ 


\section{BROBECK CORPORATION}

1235 TENTH STREET • BEPKELEY, CA $94710 \cdot(415 ; 524.8664$

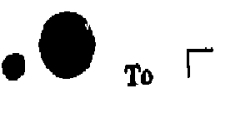

- DIAMOND TOOL CO.

5085 29ch Avenue

Oakland, CA 94605

BROBECK CORPORATION

1235 Tenth Street

Berkeley, CA 94710

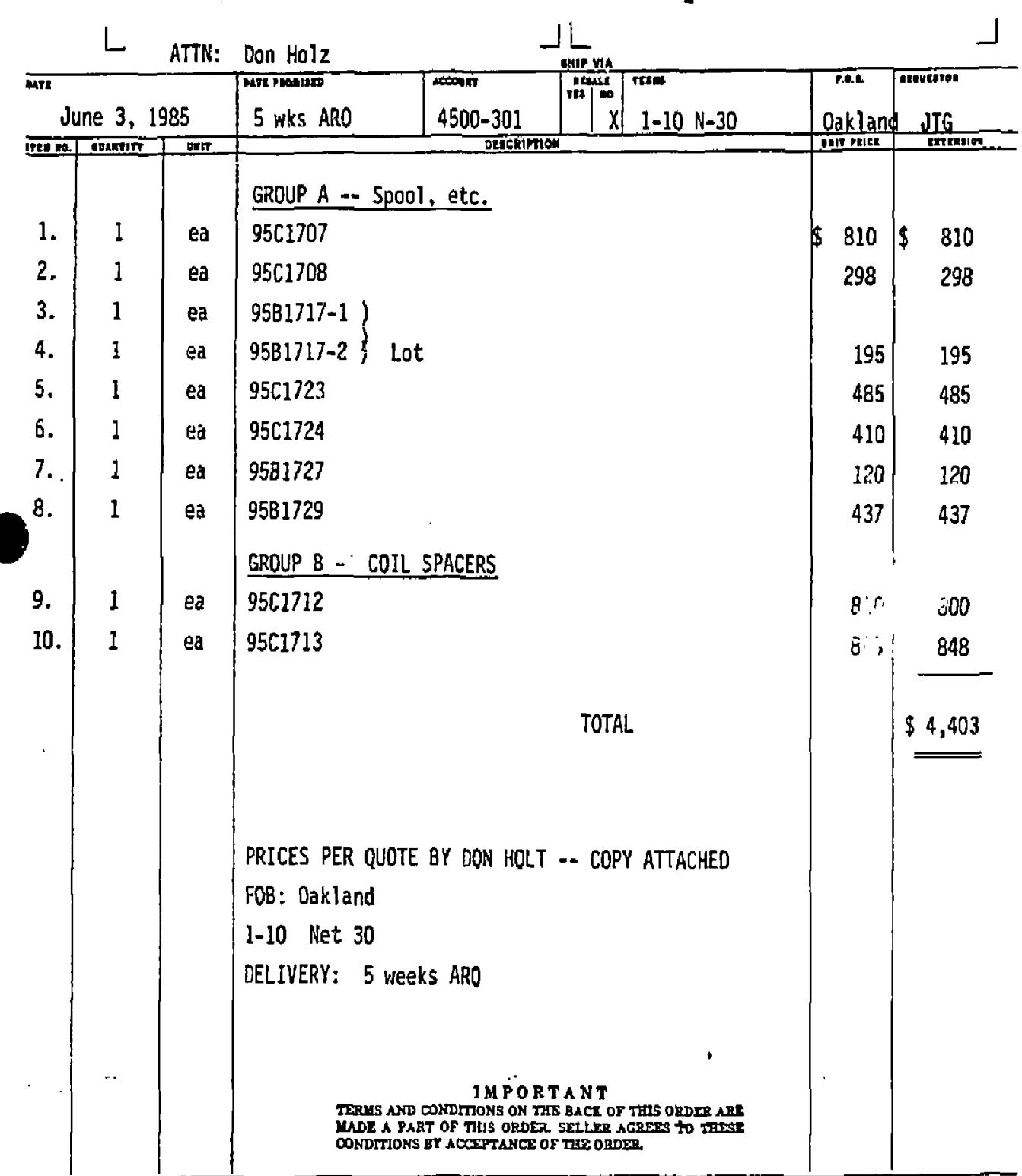

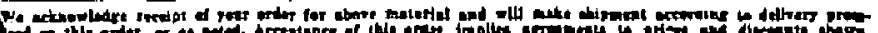

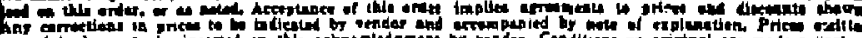

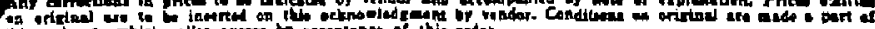

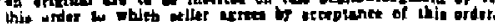

VENDOR BY

By Original signed by:

$$
\text { K. M. Thomas }
$$




\section{WILLIAM M. BROBECK \& ASSOCIATES}

RESEARCH -DEVEI GENT - ENGDNEERING $\triangle 0 B$

Sivis:

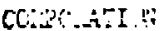
INDUSTRY - BUSLNESS - SCIENCE

1235 Tenth SL. Berkeley, CA 94710 (415) 5248664

PURCHASE ORDER

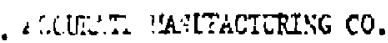

SHIP AND BILL TO

177J SA: PI:ELO AVE.

1235 Tenth Street

IATIILE, CA $9460 \%$

Berkeley, CA 94710

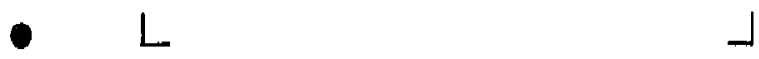

\begin{tabular}{|c|c|c|c|c|c|c|c|}
\hline 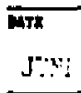 & $1: 1: 1 ;$ & & mivr. 74.1995 & 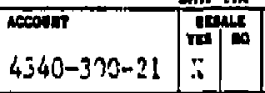 & NET 31 MU'S & B.9. & J. T. . C . \\
\hline artong. & aruminer & Eim & \multicolumn{3}{|c|}{ orechilfion $=1$} & mife nicas & Exrentom \\
\hline 1 & $L^{*}$ & & \multicolumn{3}{|c|}{ 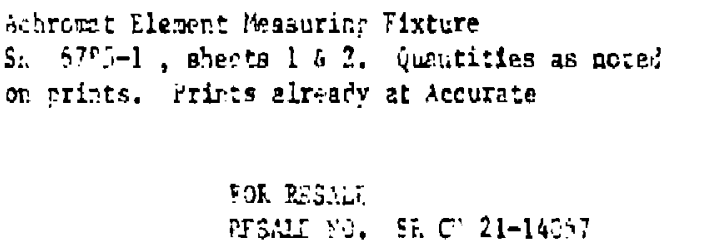 } & & \\
\hline & & & & 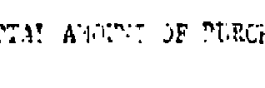 & yer & & $\begin{array}{c}\$ 1,600.00 \\
\text { LOT }\end{array}$ \\
\hline
\end{tabular}


WILUIAM M. BROB TK \& ASSOCLATES RESEARCH - DEVELOL-WENT - ENGLNEERING

$3 \times 001$

FOR

CCRBSA:D

INDUSTRY - BUSLNESS - SCIENCE

I2JS Tenth Si, Butkeley, CA 94710 (415) 524.8664

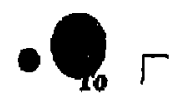

- PI RELLLTIER, INC.

SHIP AND BILL TO

$1844 x$. REYTST AVE.

2235 Teath Street

Bezekivi, Co 31504

Berkeley, CA 94710

TOt Bar iLA

RESAIL MO. ST C: 21-14\$317

MrtiL ENELT UP PO.

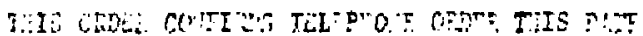

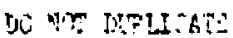

IH P O B T A T

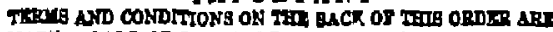

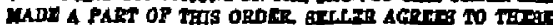
CONDITLNB HY ACCOPTAYCT OP TED ORDIP

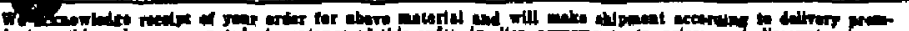

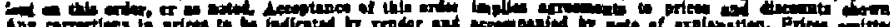
-

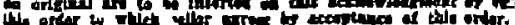

PULLAM H. BROBECE \& ASSOCIATES BP $>1 /$
나는

The HuMlain wutr Amern

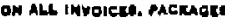
ANQ HATPINA PAPETE.

PURCHASE ORDER 
LL ITEMS PAINTED IN RED MUST •

BE FILLED IN BY REQUISITIONEA

PURCHASE REQUISITION

$\infty$

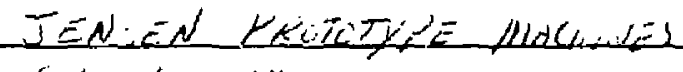

VENDOA

HilC $\angle E N Q N Q T$

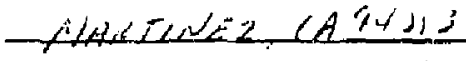

$a^{T T N}$ OF

$-226-26 x$

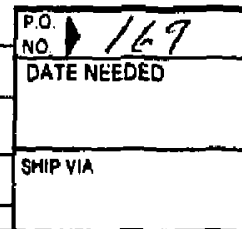

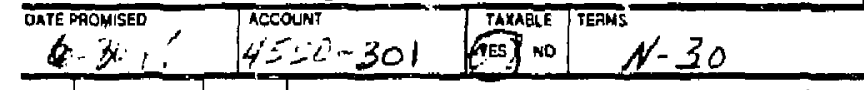

F.0.

OISPOSITION

TYPE P.O. I MAIL

ORDEA WIAED

DRDER TELEPHONED

OAME

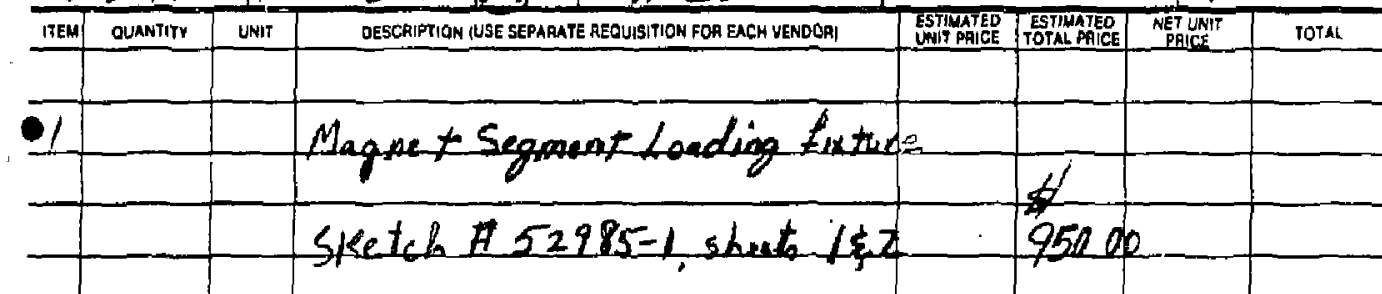

2 


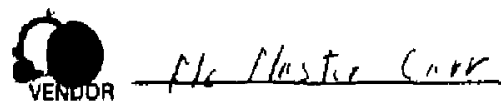
ADDRESS

\begin{tabular}{|c|c|c|}
\hline PO. 172 & $\checkmark$ & DISPOSITION \\
\hline DATE NEEDED & & TYPE P.O. \& MAIL \\
\hline \multirow[t]{2}{*}{ SHIP VIA } & & OROEA WIRED \\
\hline & & ORDEA TELEPMONED \\
\hline
\end{tabular}

ATTN. OF

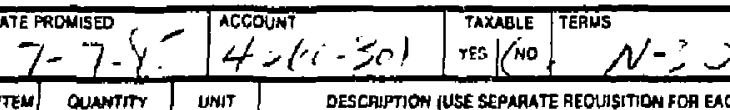

$-y-j-j-$

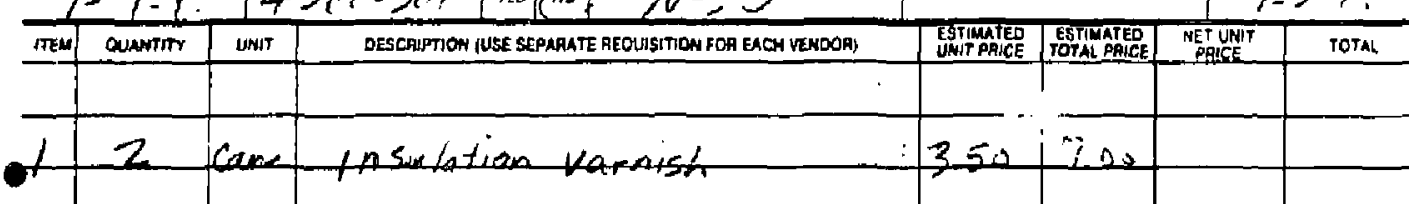

ESTIMATED TOTAL VALUE OF ORDEF MUST BE STATEO BY REOUISITIO

Suggested Sources

\section{$-1437 k 16$}

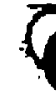

Prevous Purchase Ordar No. 
WILLIAM M. BROBECK \& ASSOCIATES

ITEMS PAINTED IN RED MUST

PURCHASE REQUISITION

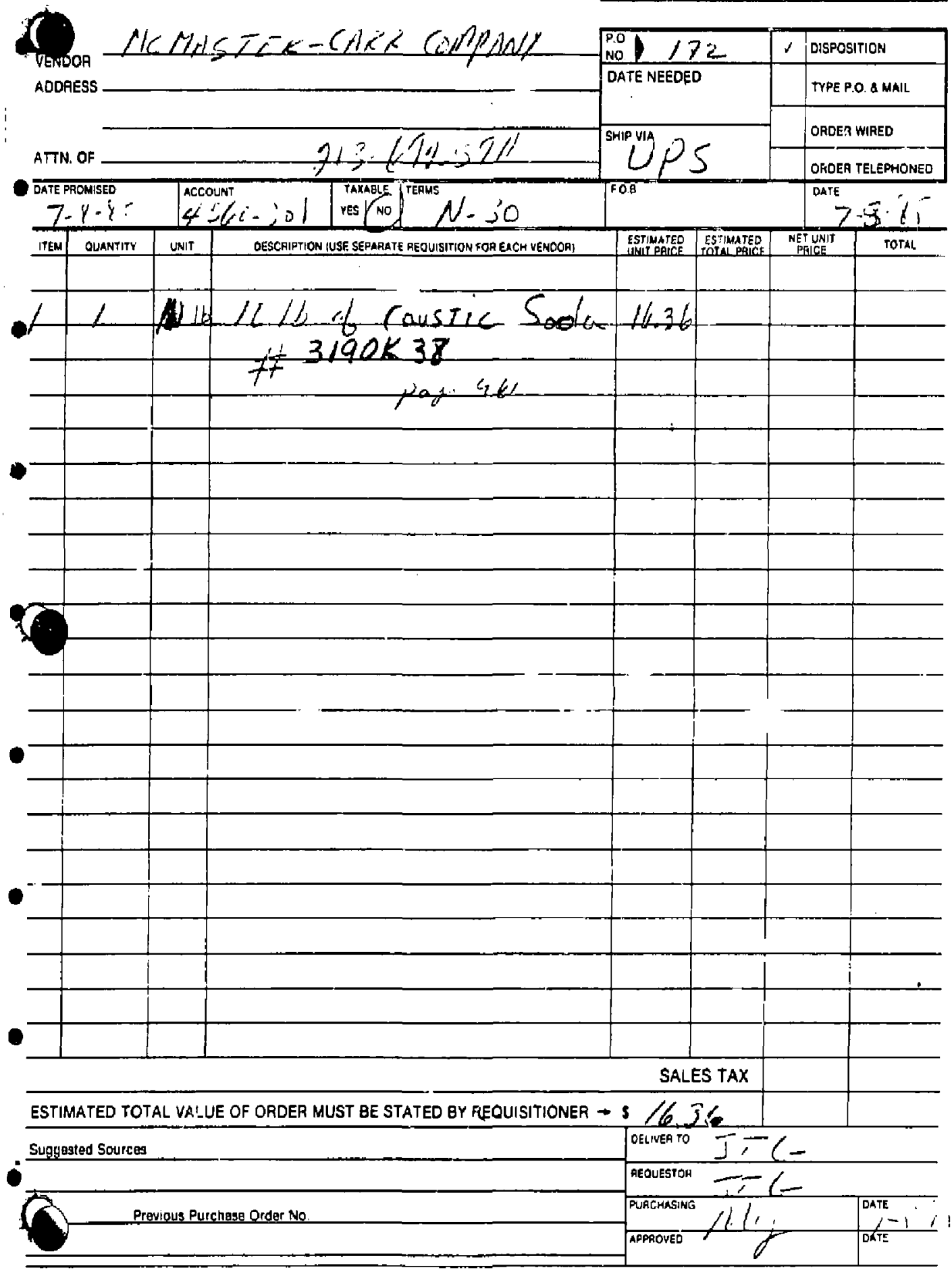

BA-WO IREV 1-79)

ACCOUNTS PAYABLE

$c-19$ 


\section{BROBECK CORPORATION}

1235 TENTH STREET • BERKELEY. CA 94710 • (415) 524-8604

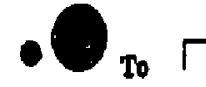

- PERHAG CO.

1159 SONONA COURT

SUNNYYALE, CA 94086

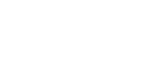

$1 L$

sule vh

\begin{tabular}{|c|c|c|c|c|c|c|}
\hline JULY 11, 1985 & Mit notus & 4540300 & 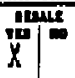 & $\mathrm{N}-30$ & R.A.2. & J.T.GUNN \\
\hline
\end{tabular}

Rework 64 permanent magnet segments by removing $0.0015^{n}$ as discussed on meeting of Jyly 17, 1985.

unt nater

\section{IMPORTANT}

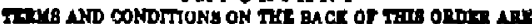

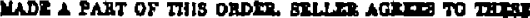
CONDFOAB EY $\triangle$ CCTPIANC OF THE ONDA. 
BROBECK CORPORATION

1235 TENTH STREET • BERKELEY, CA \$4710 • (4IS) 524-8664

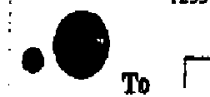

- electrical specialties co.

SAN FRANCISCO, CA

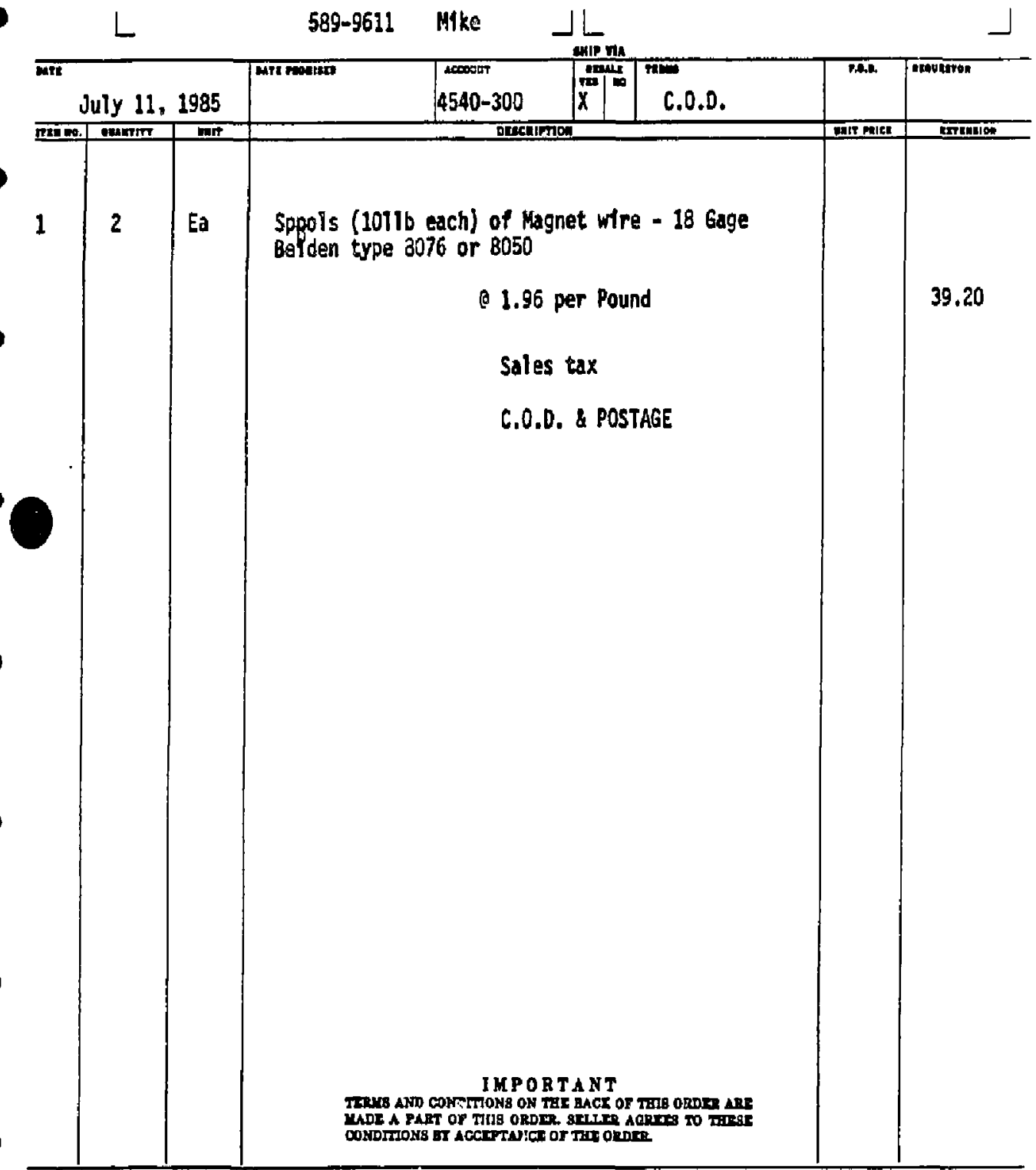

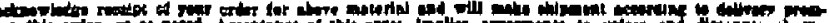

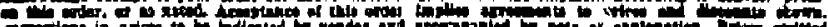

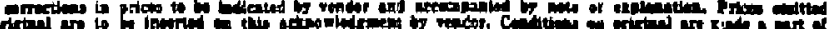

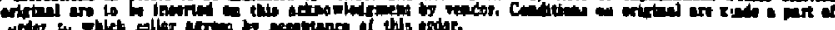

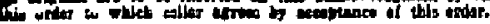

VENOOR EY

WTIIL.

ALUE: Rerenestor

GREEN: Prigst File

YELLOW: Vendor Actnowled thment

PINK: Purthume'Shipping

GOLD: Actownting thit183내내

TMI: Mumato must abpean ANAL INrolter. MACMAOES

PURCHASE ORDER

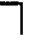




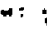

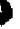 \\ $=$ \\ BROBECK CORPORATION}

I235 TENTT STREET • BERKELEY. CA 94710 • (\$|5) 524-8664

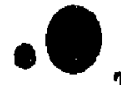

To $\Gamma$

- PERMALLUSTER, INC.

1644 N. Keystone Ave.

Burbenk, CA 91504
BROBECX CORPORATION

1235 Tenth Street

Berkeley, CA 94710

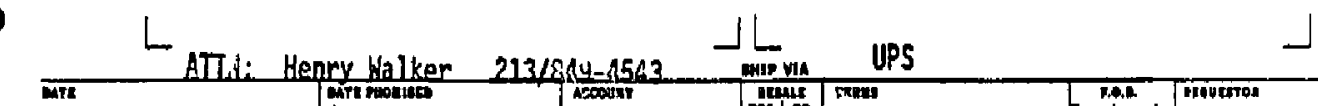

$11-25-85$ (1)

1.

it. $12-7-85$ $4500-301-20$ rat $\mid \begin{array}{ll}\mathbf{x} \\ \mathbf{x}\end{array}$ DECAIFION

Net 20
Spobeck

\author{
Annea $7 e d$, rounded edges, free of burrs
}

TOTAL AMOUNT OF PURCHASE OREER

$\$ 625.83 /$ Lot.

SHIPMENT HUST BE MADE WITHIL 5 WDRKING DAYS OF THE RECEIPT OF THIS ORLCR AND, IIN NO CASE WILL IT BE ACCEPTED LATER THAN 12-10-85.

C.O.D. SHIPMENT UILL HOT BE ACCEPTED ON ANY CONDITIOIS.

IMPORTANT

FENA AND CONDTHONB ON TAE BACE OF THIS OWOBT ARE

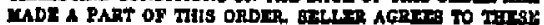

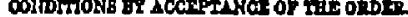

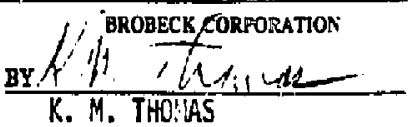

\title{
VENDOR BY
}


WILLIAM M. BROBECK \& ASSOCIATES

$\triangle$ ITEMS PRINTED IN RED MUST BE FILLED IN BY AEQUISITIONEA

PURCHASE REQUISITION

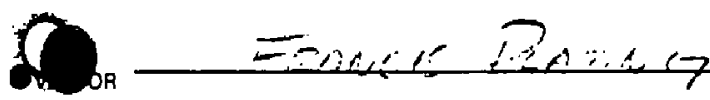

ADORESS

ATTN. OF

DATE PROMISED

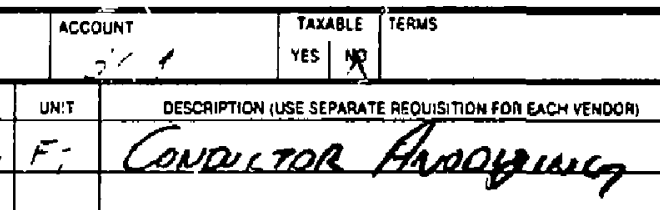

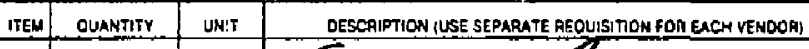

$1 \int_{1}^{2}: F$

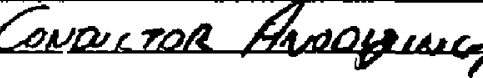

\begin{tabular}{|c|c|c|}
\hline \begin{tabular}{l|l} 
P.O & 197 \\
No & 19
\end{tabular} & $\checkmark$ & DISPOSITION \\
\hline DATE NEEDED & & TYPE P.O. \& MAIL \\
\hline \multirow[t]{2}{*}{ SHIP VIA } & & ORDER WIAED \\
\hline & & ORDER TELEPHONED \\
\hline F.0.8. & & $5,2 \cdot M .5$ \\
\hline
\end{tabular}

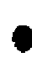

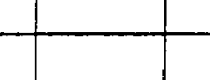

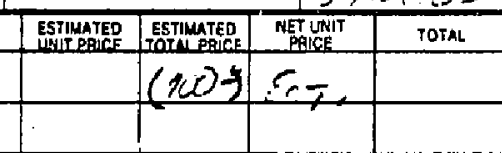
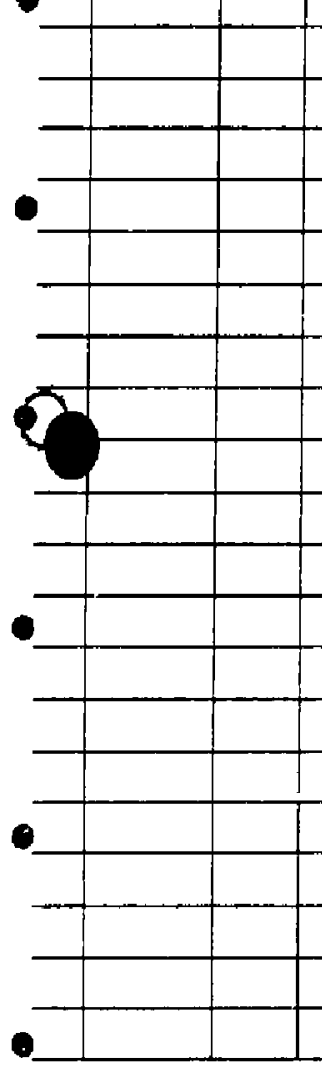

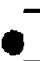

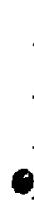


ALL ITEMS PRINTEO IN RED MUST BE FILLED IN GY REQUISITIONEA

PURCHASE REQLISITION

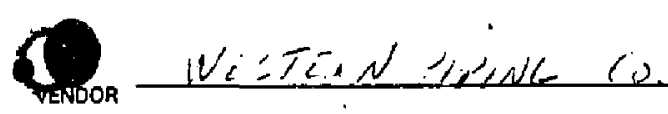
i ADDRESS

ATTN. OF

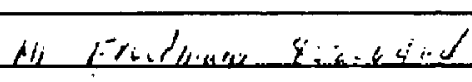

\begin{tabular}{|l|l|l|}
\hline $\begin{array}{l}\text { P.O } \\
\text { NO. }\end{array} 2 / 6$ & $\checkmark$ & DISPOSITION \\
\hline OATE NEEDED & & TYPE P.O. \& MAIL \\
\cline { 2 - 3 } & & ORDER WIREO \\
\hline SHIP VIA & ORDER TELEPHONED \\
\hline
\end{tabular}

\begin{tabular}{|c|c|c|}
\hline DATE PAONISED & $\begin{array}{l}4=4:-301 \\
\text { ACCOUNT }\end{array}$ & 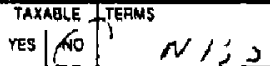 \\
\hline
\end{tabular}
$7-4-11$

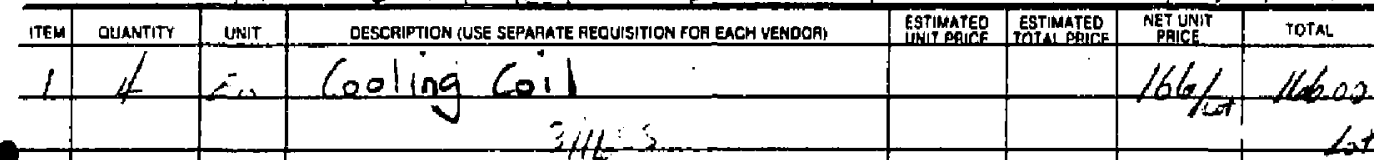

\begin{tabular}{l|l|l|l}
\hline & & & \\
\hline
\end{tabular}

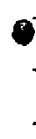

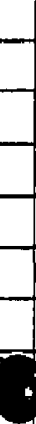

$$
+
$$

$$
+
$$

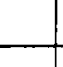

ESTIMATED TOTAL VALIJE OF QRDER MUST BE STATED BY REQUISITIONER $\rightarrow$

\section{sug}

Suggested Soutces

Previous Purchase Drder No 


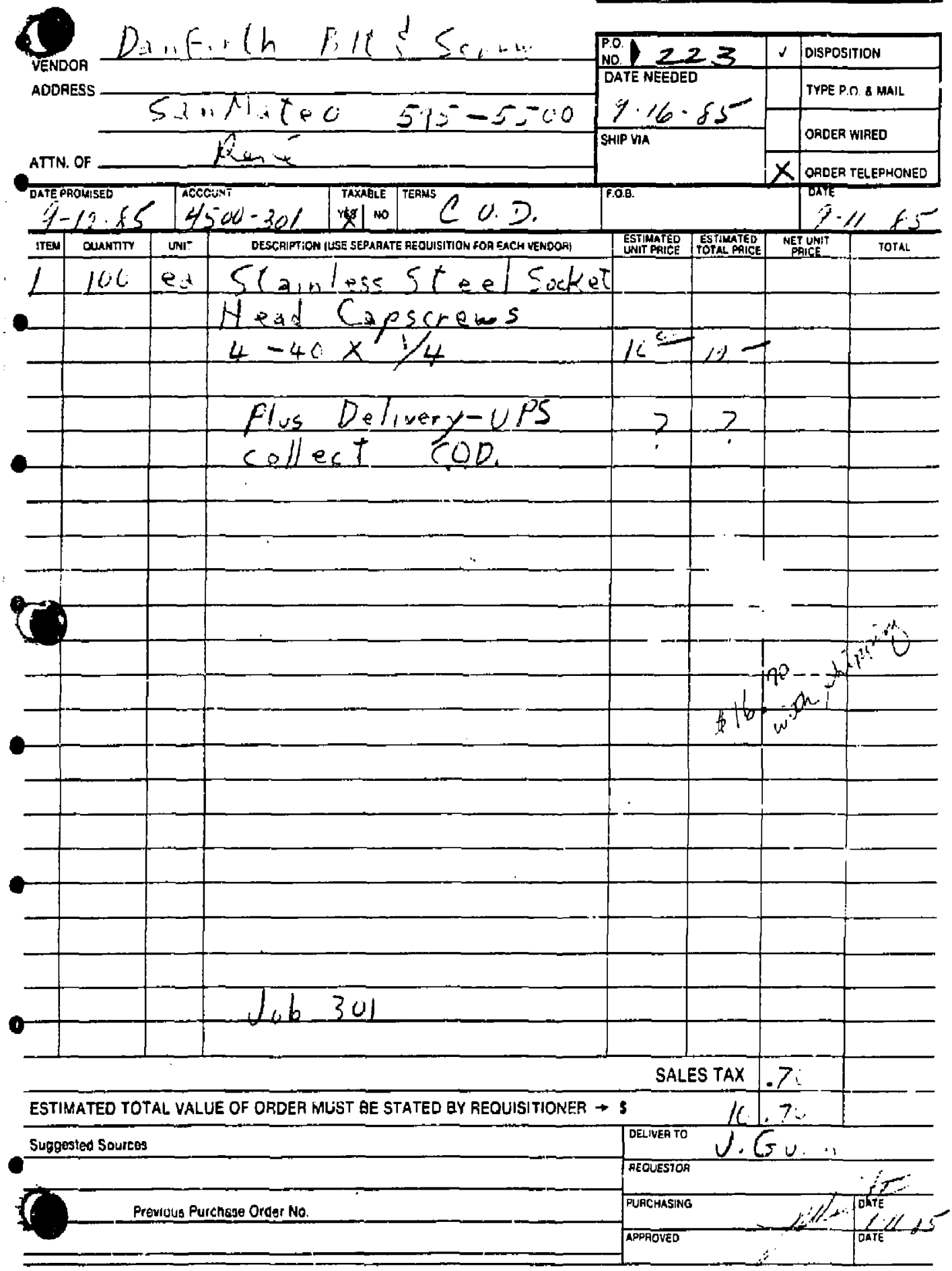




\section{BROBECK CORPORATION}

1235 TENTH STREET - BERKELEY, CA 94710 - (415) 524-8664

i

- acCuRATE manufacturing co. 4770 San Pablo Ave.

Emeryville, CA

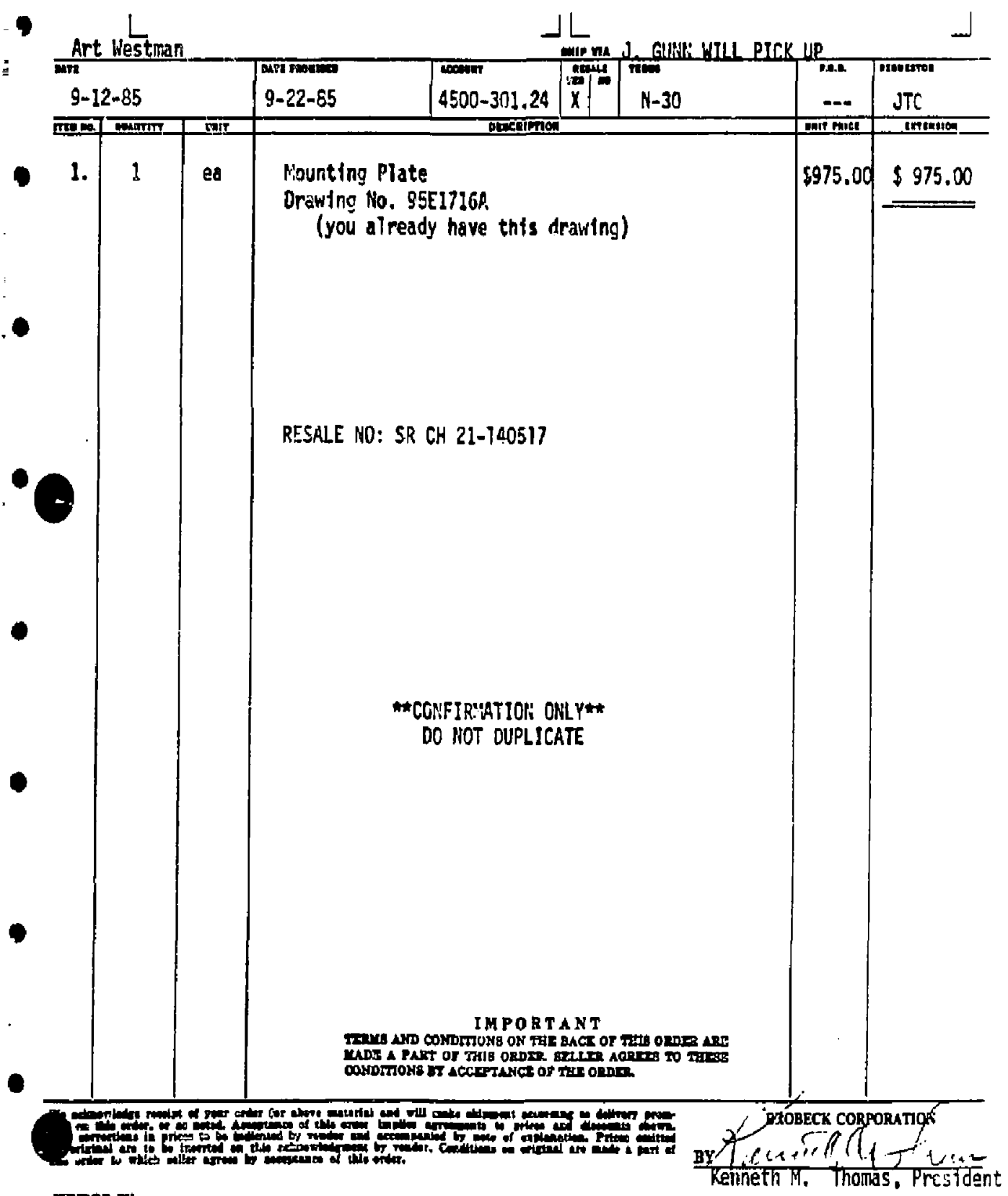

VMDOR BY

watre: Veroto 
WILLIAM M. BROBECK \& ASSOCIATES

ALL ITEMS PRINTED IN RED MUST

BE FILLED IN BY REQUISITIONER

PURCHASE REQUISITION

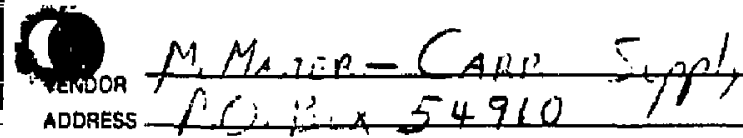

ADDERS COS 54960
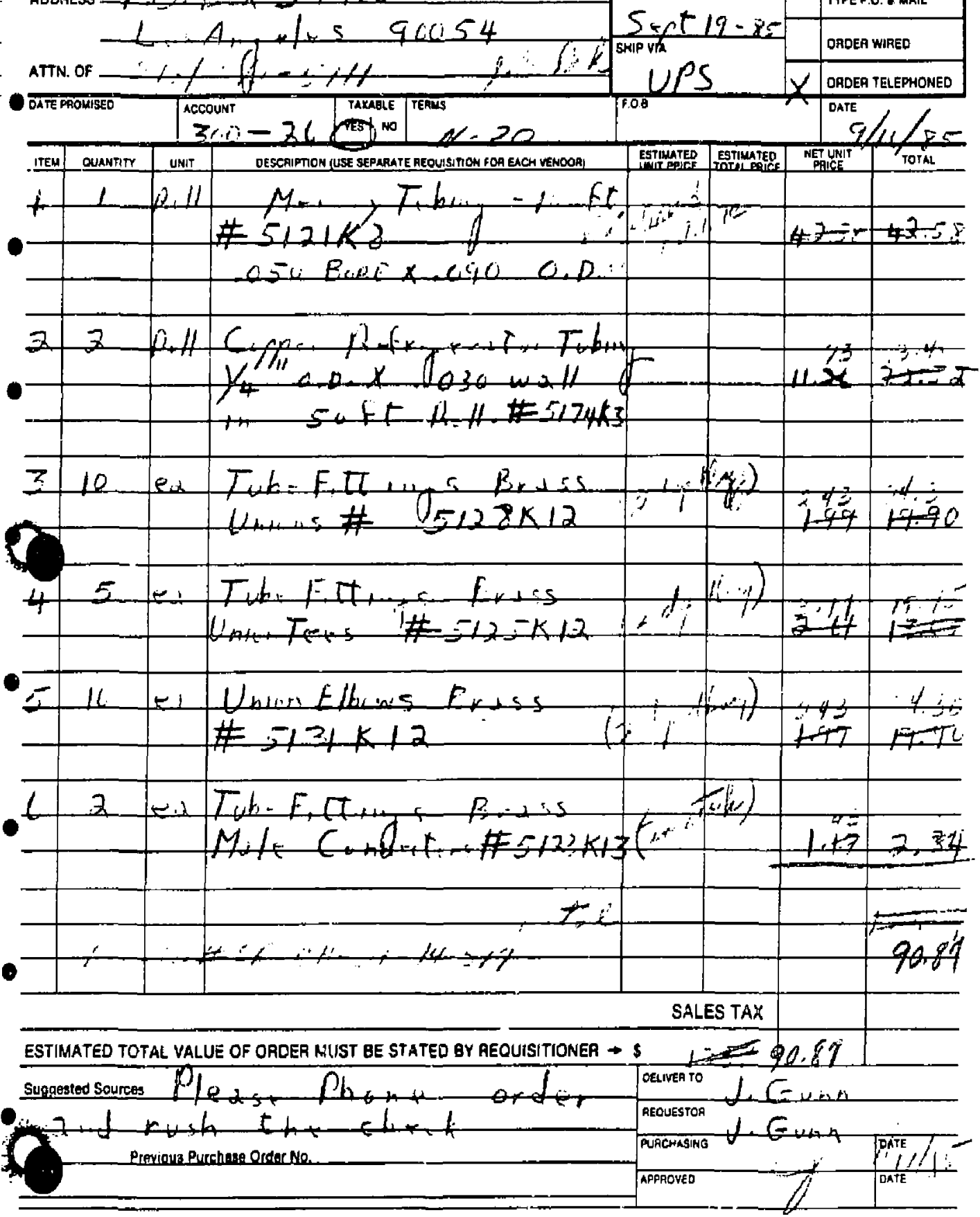

E-730 (REV. I. TO)

REQUISITIONER

C- 2 ? 


\section{BROBECK CORPORATION}

I235 TENTH STREET • BERKELEY. CA $\$ 4710$ • (415) 524-8664

$\cdot \boldsymbol{\theta}_{\mathrm{w}} \mathrm{r}$

Ship to:

\section{6}

twis mumber wurt arresh ow All inroucre, mackars

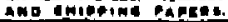

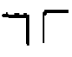

- HESTERN PIPE \& ENGINEERING CO.

1485 Yosenite Ave.

South San Francisco, ca $94 / 24$

BROBECK CORPORATION

1235 Tenth Street

Berkeley, CA 94710

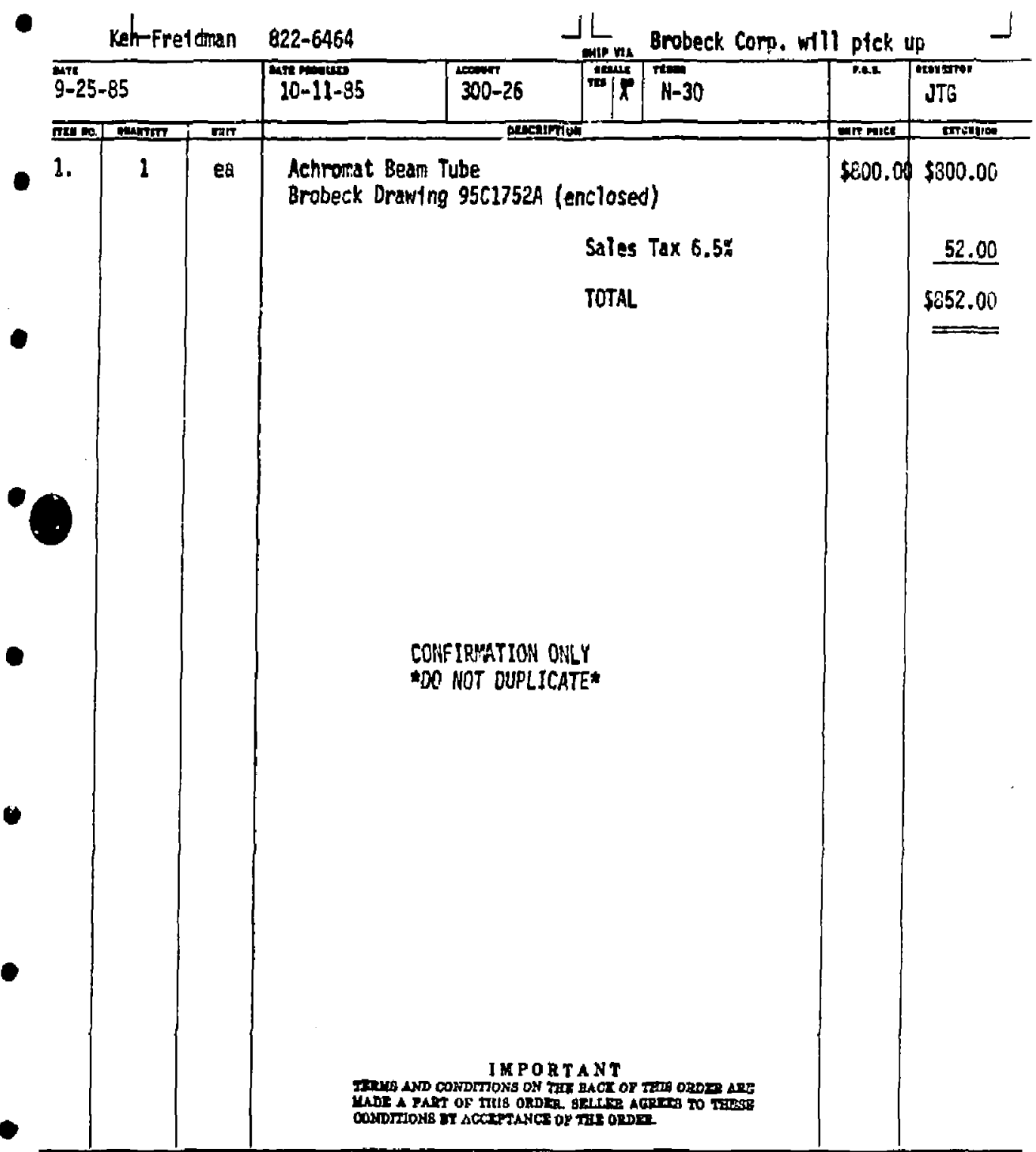

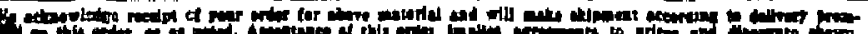
C tw

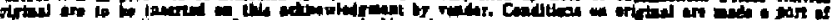

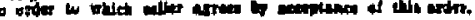

\section{VExpon EY}

1 BROAECK CORPORATION

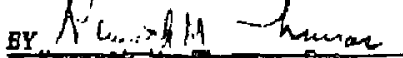
keimeti H. Truthas, Pres. 


\section{BROBECK CORPORATION}

1235 TENTH STREET • BERKELEY. CA 94710 • (415) \$24.8654

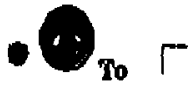

- permag sierra corp.

1159 Sonora CE.

Sunnyvale, CA 94086

\section{BROBECK CORPOPATION}

1235 Tenth Strect

Berkeley, CA 94036

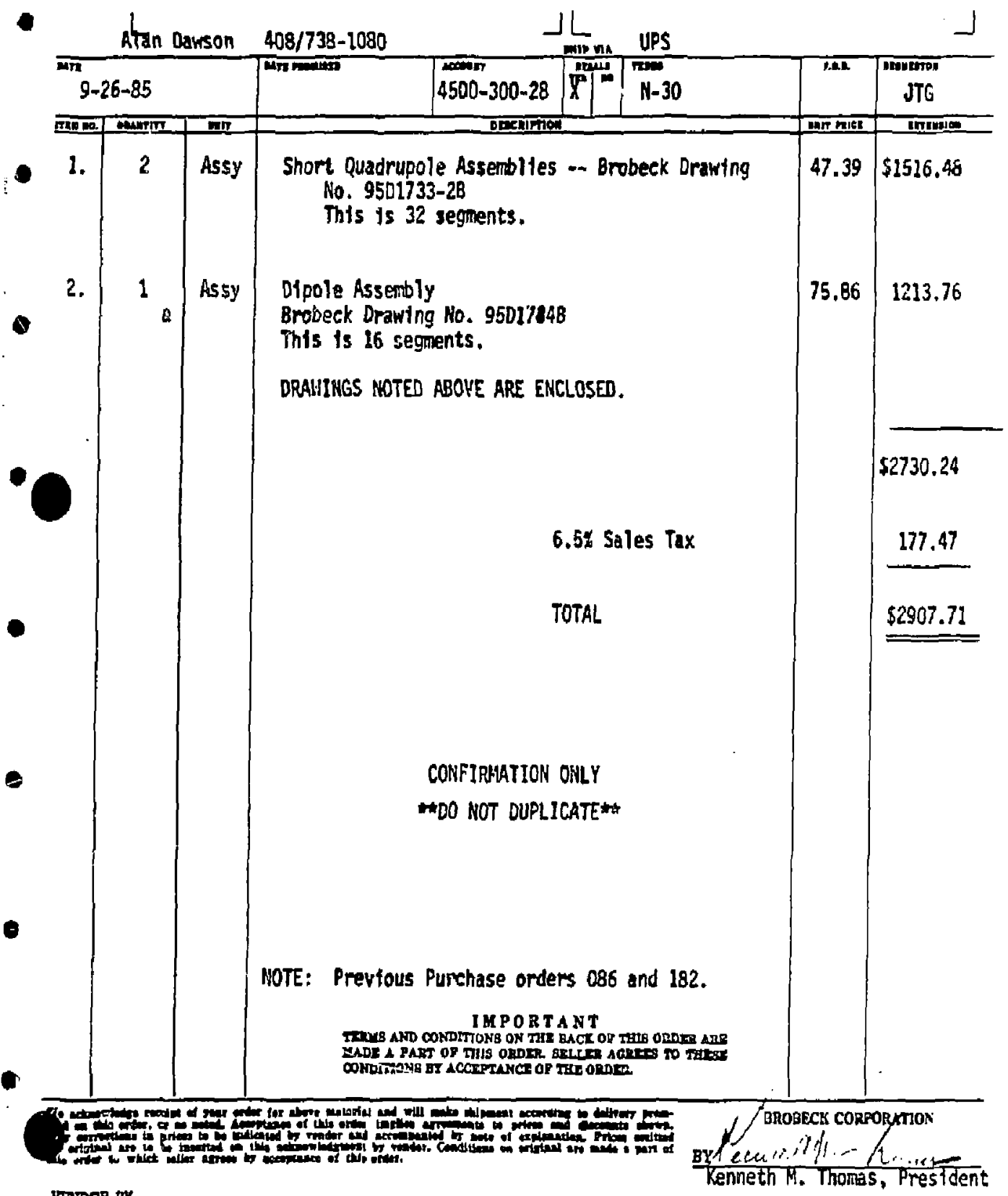




\section{BROBECK CORPORATION}

1235 TENTH STREET * BERKELEY. CA 94710 - (415) 524-8664

$\because{ }^{\circ}$ To $\Gamma$

- MDC CORPORATION 23542 Cabot Bivd. Hayward, CA 94545

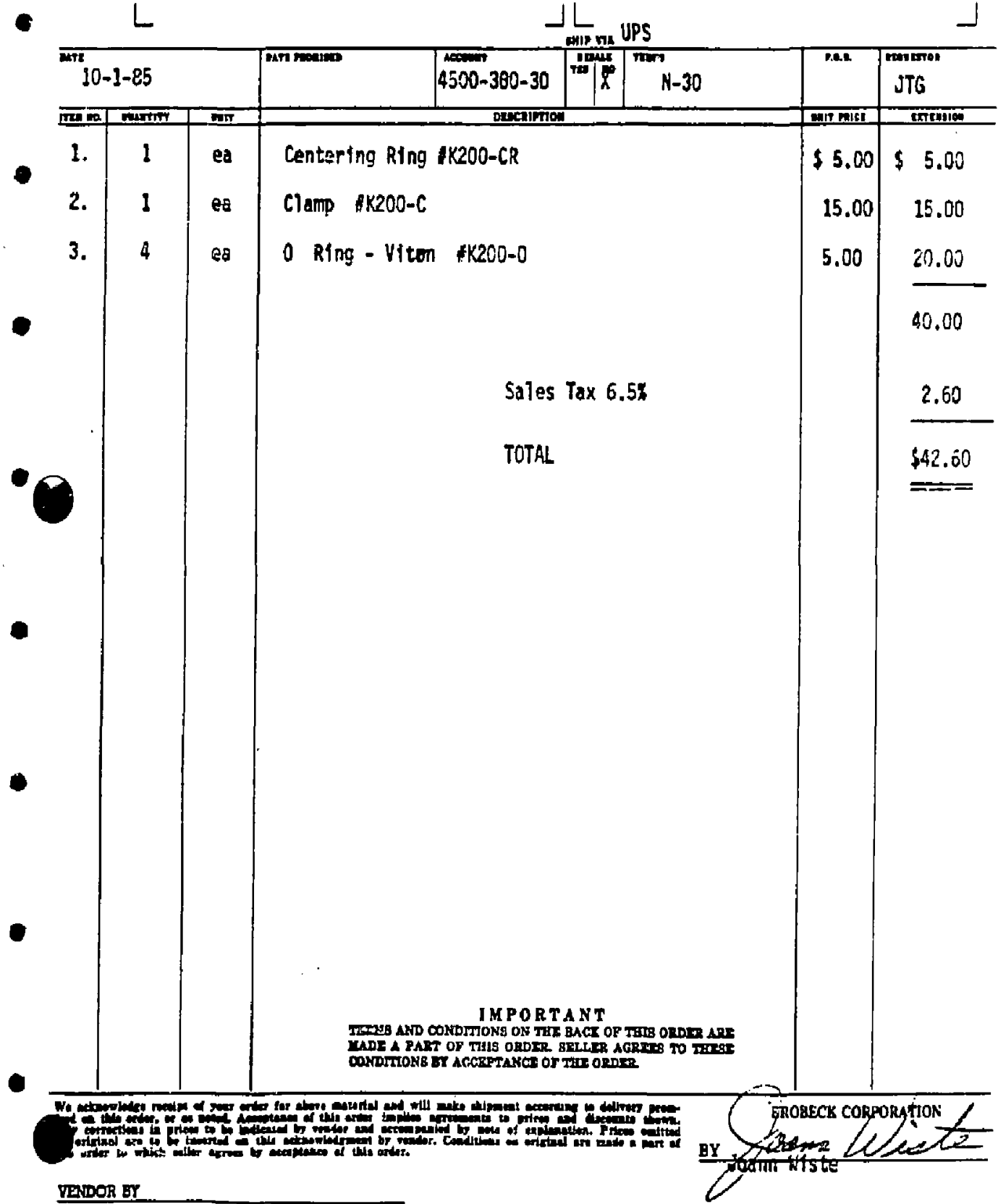




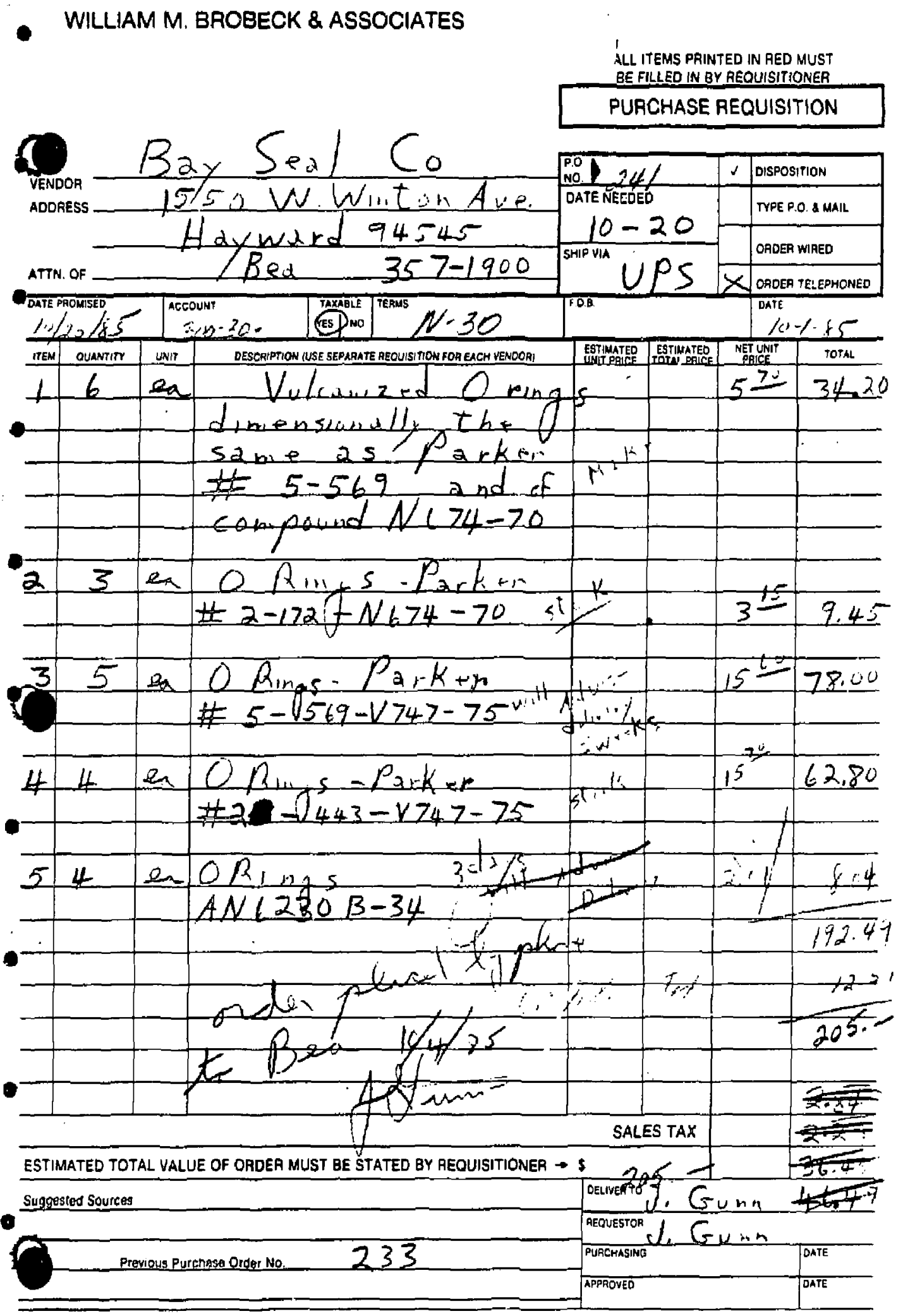

6A-10 [REV :-TO]

ACCOUNTS PAYABLE

C -35 


\section{BROBECK CORPORATION}

1235 TENTH STREET • BERKELEY, CA 94710 • (419) \$24-8664

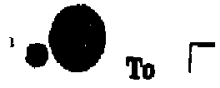

- cMEgA ENGR. INC.

Box 4047

Stanford, CONM. 0690?

PURCHASE ORDER

BROBECK CORPORATION

1235 Tenth St.

Berkelsy, CA 94710

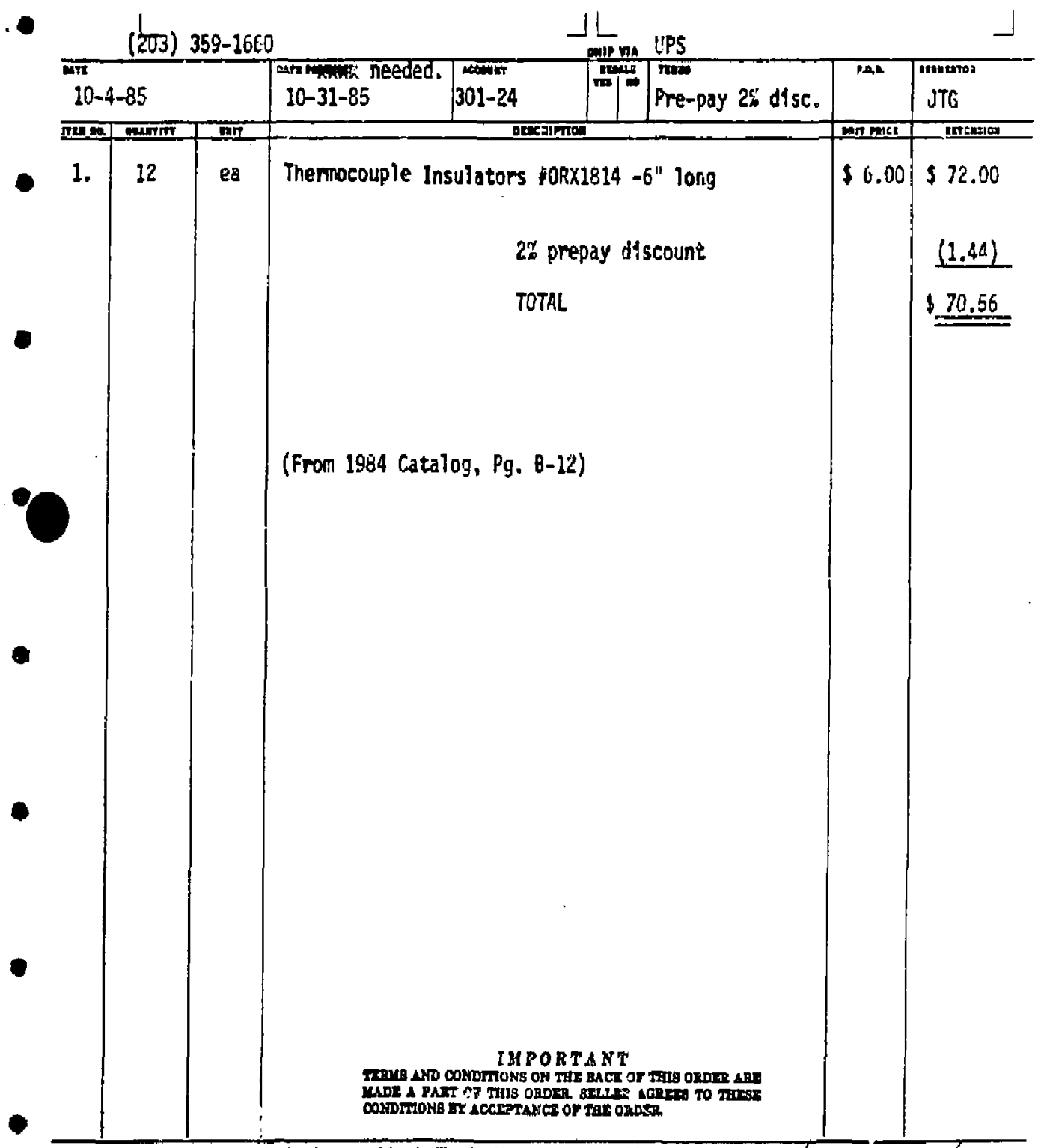

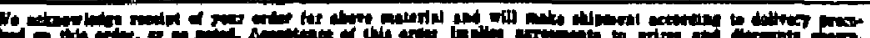

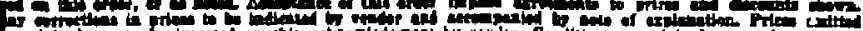

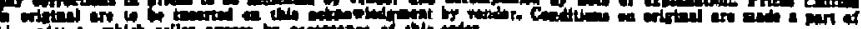

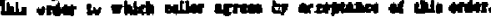

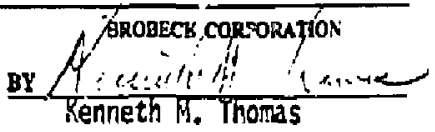

VERDOR BY 


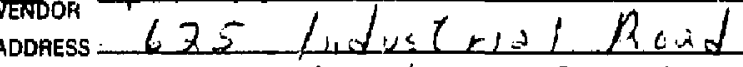
San Mareo, Col, ATTN OF $595-5-00$

\begin{tabular}{|c|c|c|c|c|c|c|}
\hline \multirow{2}{*}{\multicolumn{2}{|c|}{ DATE PROMIISES }} & \multirow{2}{*}{\multicolumn{2}{|c|}{ 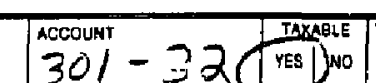 }} & B. & \multirow{2}{*}{\multicolumn{2}{|c|}{$10 / 4 / 85$}} \\
\hline & & & $1-320$ Yes jo NeI & & & \\
\hline ITEM & Quentiriv & UNIT & 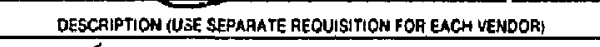 & 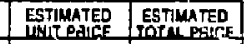 & 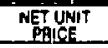 & TOTAL \\
\hline 1 & 100 & 2 & $5 / 8$ 5.5. wa 5/4mes & & & $\operatorname{loc}$ \\
\hline 2 & $\mu 0$ & e. & $3 / 8 \quad 11$ & & & 17.80 \\
\hline 3 & 25 & a & $5 / 2-11 \times 3^{11}$ Hexhol.55. & & & 35.00 \\
\hline$\underline{4}$ & 50 & مبتر & $35 \times 3$ twokl ss & & & 23.20 \\
\hline 5 & 50 & \& & $3 / 2 \times 11 / 4$ & & & $11.5-$ \\
\hline$\ell$ & 110. & ea & $5 \%-11 \quad N_{u} X_{s}$ & & & 32.50 \\
\hline 7 & 110 & st & $\mu-40 \times 1 / 4, Q . H, 55$ & & & 1.27 \\
\hline 8 & 100 & $\underline{\underline{2}}$ & $5-40 \times 1 / 25 c k k+1 / 15 s$ & & & 14,70 \\
\hline 9 & 100 & ع & $(-32 x+1 / 4 \quad$ a $\quad 55$ & & & $13.4 i$ \\
\hline 10 & 50 & 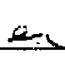 & $3 / 8-16 \times 1 \quad 11$ & & & 18.90 \\
\hline & 50 & en & $32-16 \quad N u r_{c}$ & & & 3.30 \\
\hline & & & & & & \\
\hline & & & & & & \\
\hline 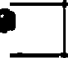 & & & & & & \\
\hline & & & Tel-pons & $0 . d+10$ & $/ 4$ & \\
\hline & & & 1 & & & \\
\hline & & & $D_{u, n} l_{1, ., t_{t}}$ & & & \\
\hline 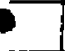 & & & & & & \\
\hline & & & & & & \\
\hline & & & . & & & \\
\hline & & & & & & \\
\hline 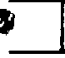 & & & & & & \\
\hline & & & 6 & 5 SALLES TAX & & 11.56 \\
\hline ESTI & IATED TOT & L VAL & E OF ORDER MUST BE STATED BY REQUISITIONER $\rightarrow$ & $\$ 119.39$ & & 189.3 \\
\hline Sugg & ted Sources & & & Deliven to & & \\
\hline & & & & REOUESTOR & & \\
\hline & & ious $P_{u}$ & age Order Nom & PuECHASIMA & & DATE \\
\hline
\end{tabular}




\section{BROBECK CORPORATION}

1235 TENTH STREET - GERKELFY, CA 91710 • (4I5) 524-8664
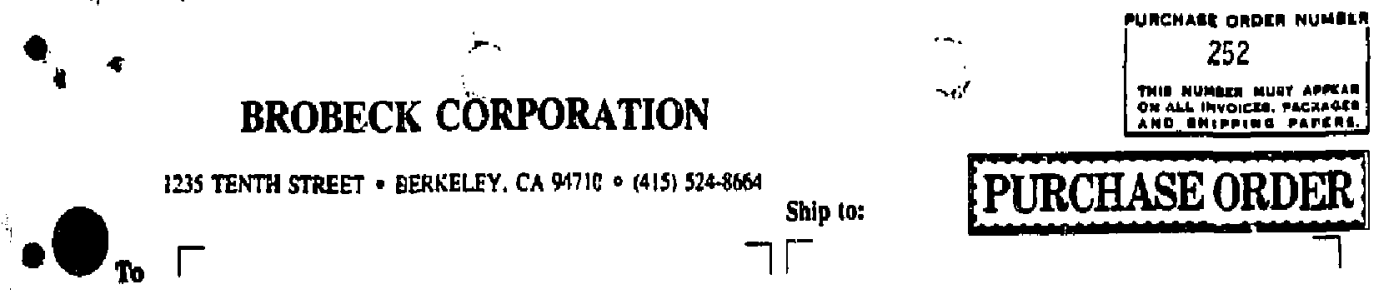

PURCHASE ORDER

- oakland valve company 2487 Estand Hay

Pleasant H111, CA 94523

BROBECK CORPORATION

1235 Tenth Street

Berkeley, CA 94710

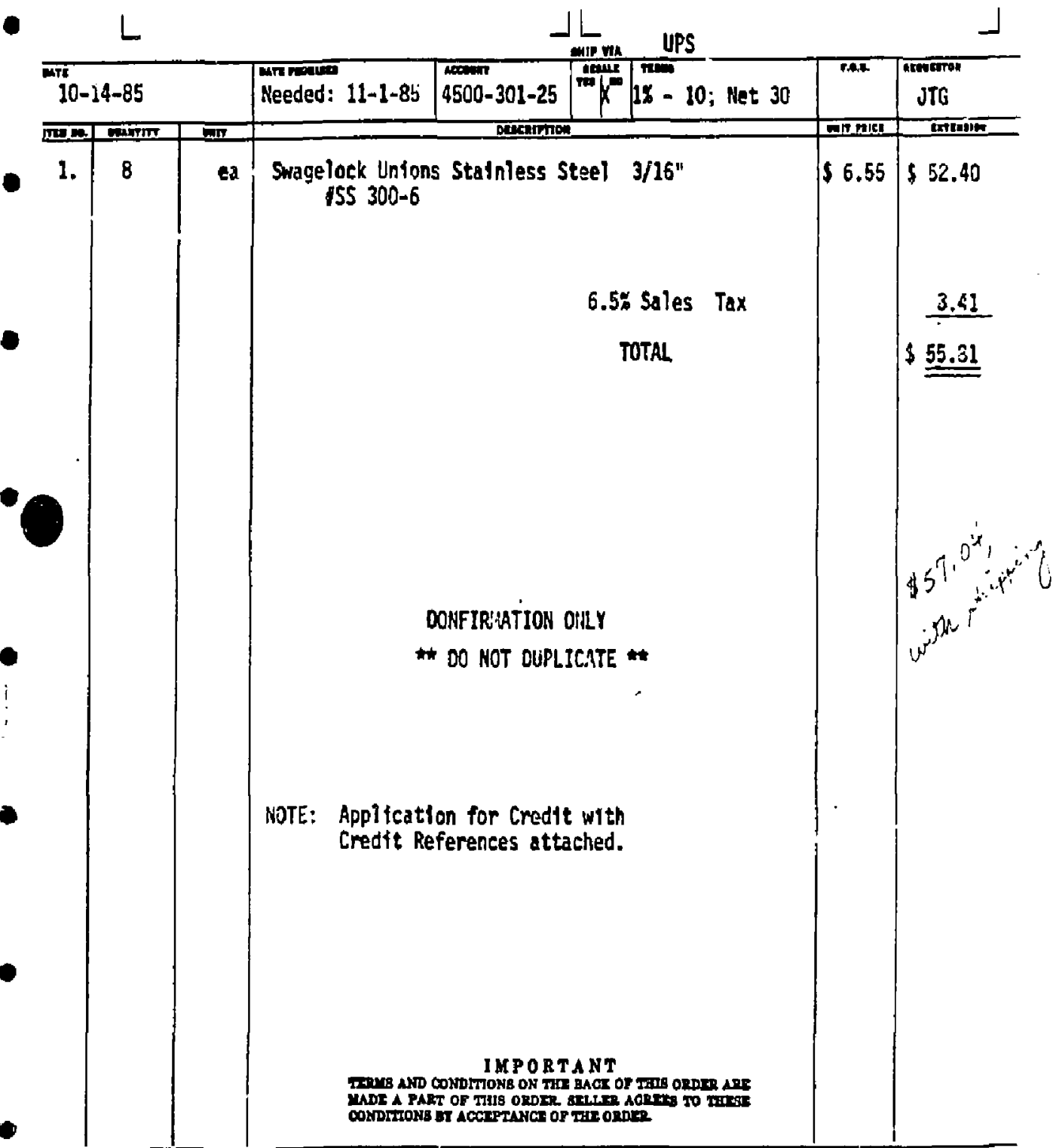

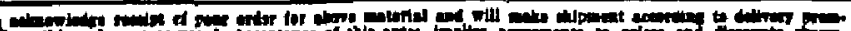

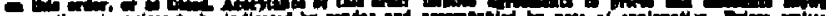

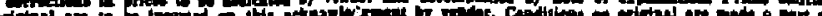
Hifla

VE, $1 \mathrm{DOR} \mathrm{BY}$

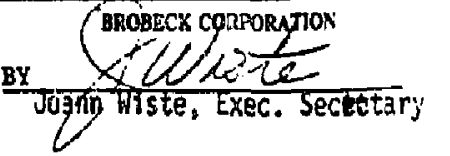

WhIt Veak 


\section{1. BROBECK CÓRPORATION}

I235 TENTH STREET • BERKELEY, CA 94710 • (415) 524-8664

$\bigcup_{\mathrm{T}_{0}} \Gamma$

- caral inr.

578 Cleveland Ave.

Albany, CI 94706
BROBECK CORPORATION

1235 Tenth St.

Berkeley, CA 94710

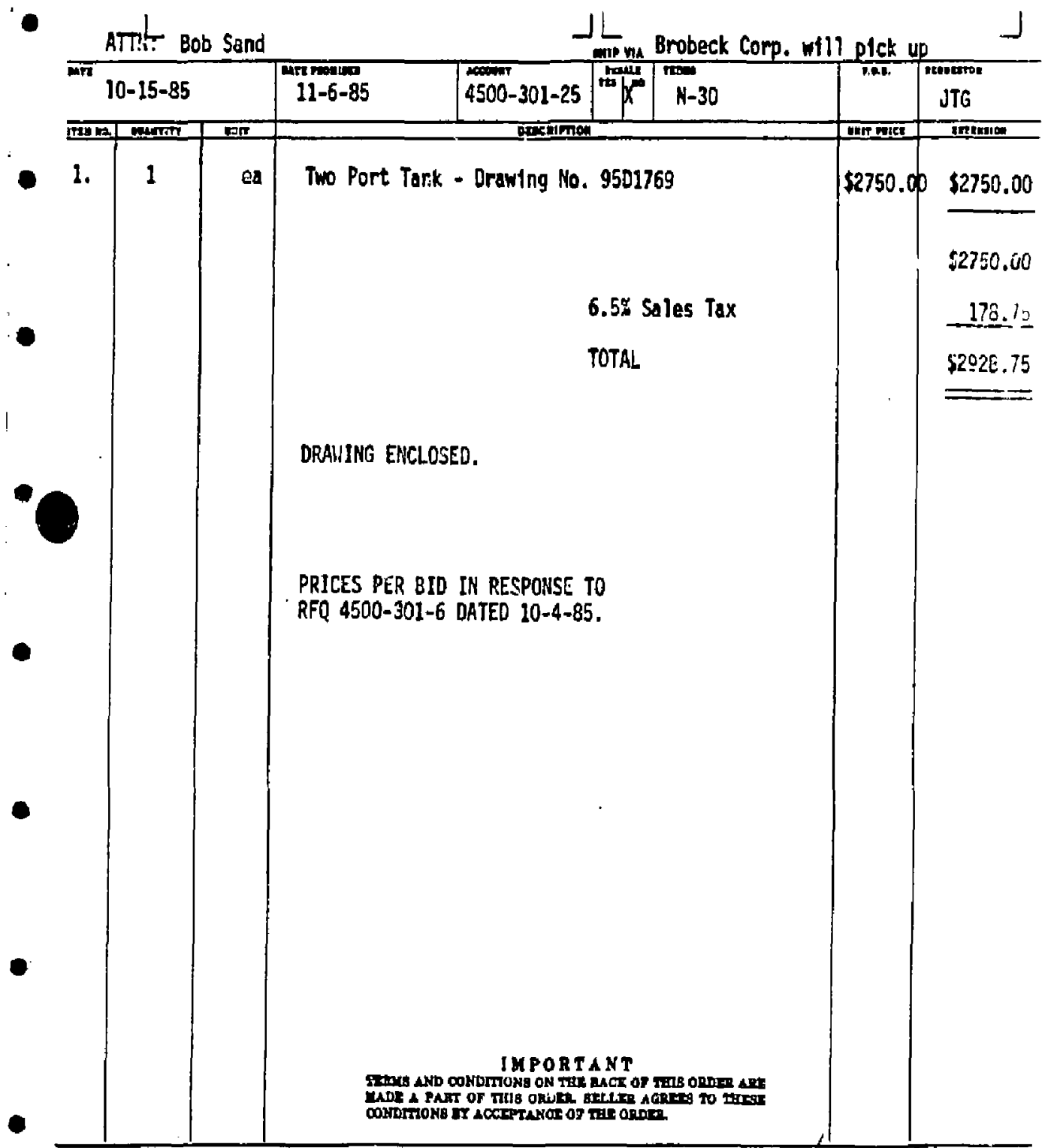

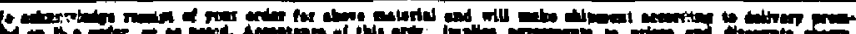
- La 10

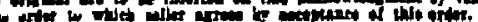

\section{VERTOA BY}

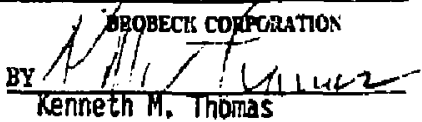




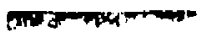

$+5$

\section{BROBECK CORPORATION}

1235 TENTH STREET * BERKELEY. CA $94710 \cdot(415) 524-8664$

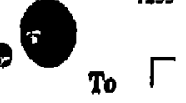

- SCHRADER SCIENTIFIC

2976 Arf Avenue

Hayward, CA 94545

\section{BROBECK CORPORATION \\ 1235 Tenth Street \\ Bekkeley, CA 94710}

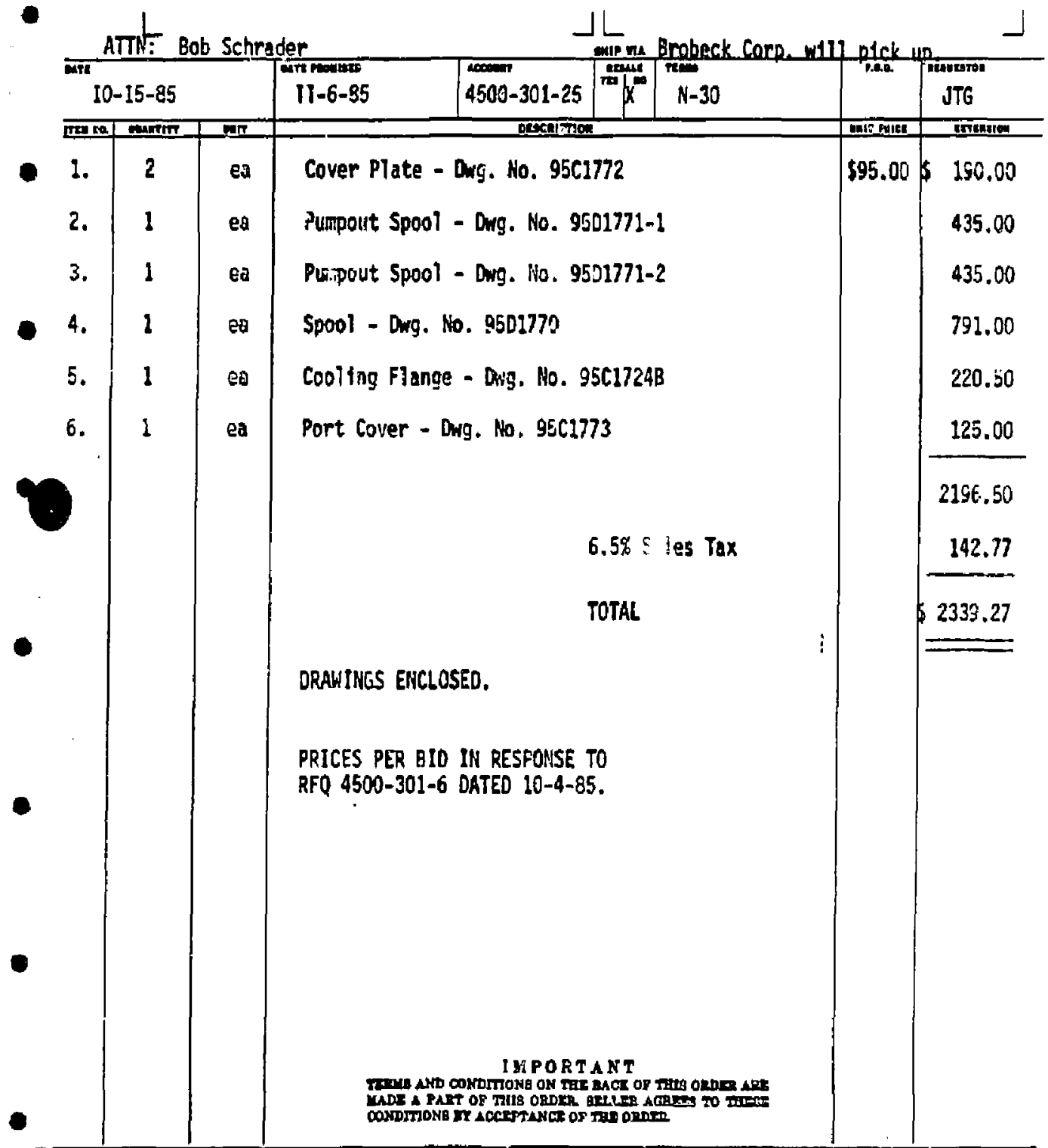

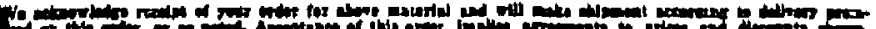

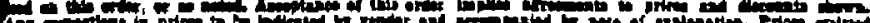

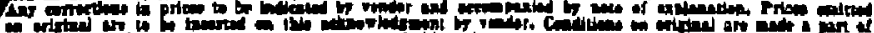

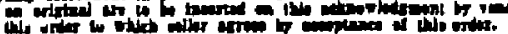

VERDOA BX

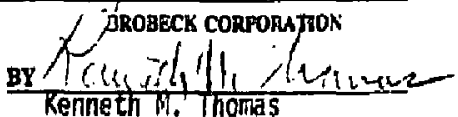




\section{BROBECK CORPORATION}

1235 TENTH STREET * BERKELEY, CA 94710 • (415) \$24-8664

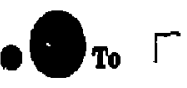

- JENSEN PROTOTYPE MACHINES

940 Laman Street

BROBECK CORPORATION

Martinez, CA 94553

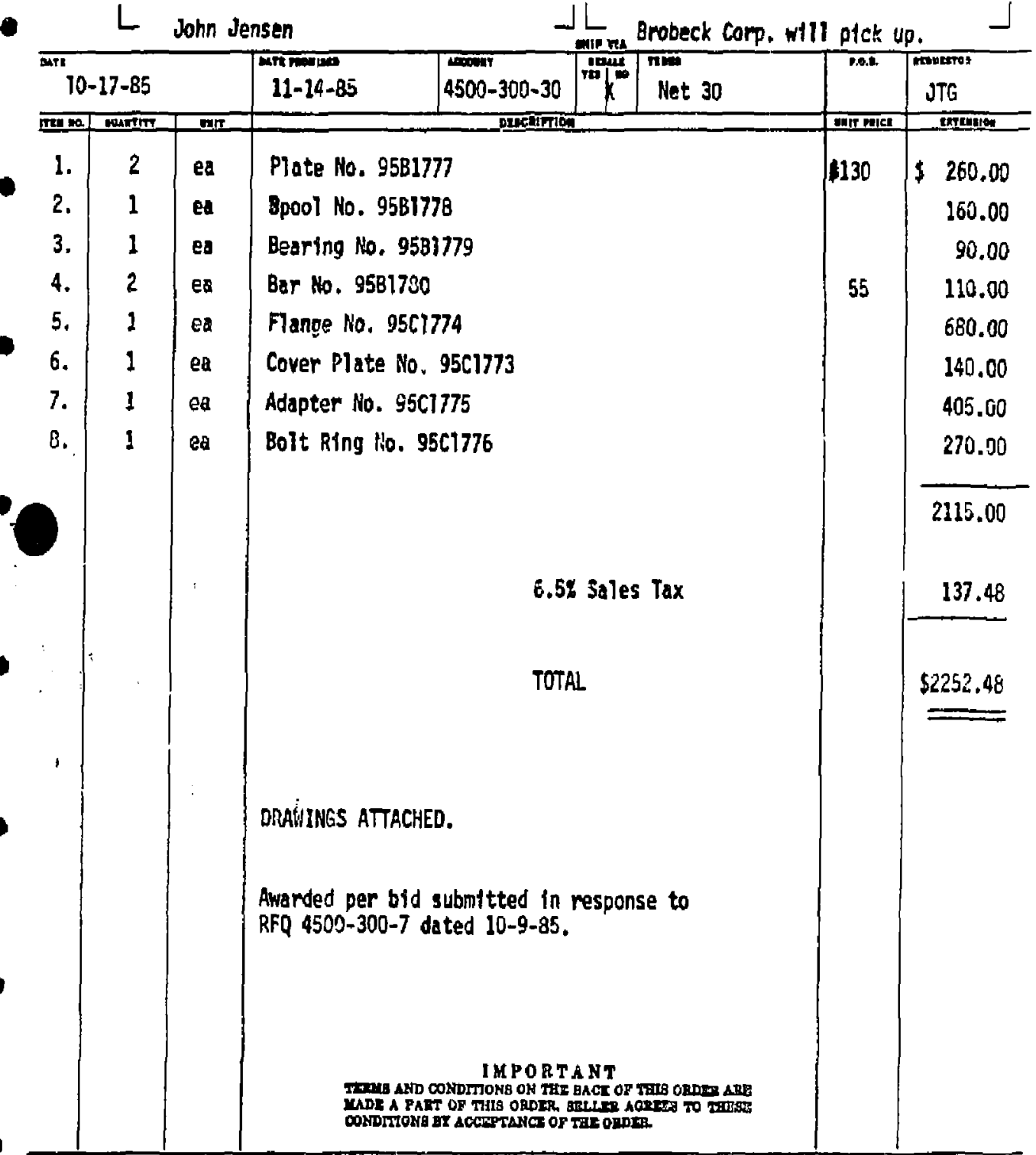

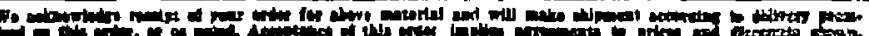

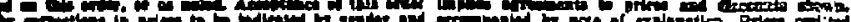

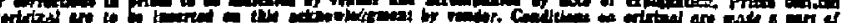

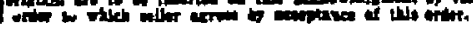

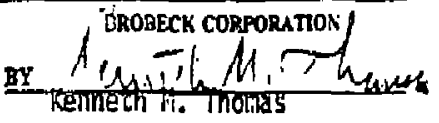




\section{BROBECK CORPORATION}

|235 TENTH STREET • BERKELEY, CA 94710 * (413) 524.6664

Ship to:
259

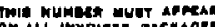

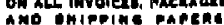

PURCHASE ORDER

\section{$7 \Gamma$}

HAND DELIVERY

- HERRIHGTON-OLSON

769 22nd Street

Qkkland, CA 94612

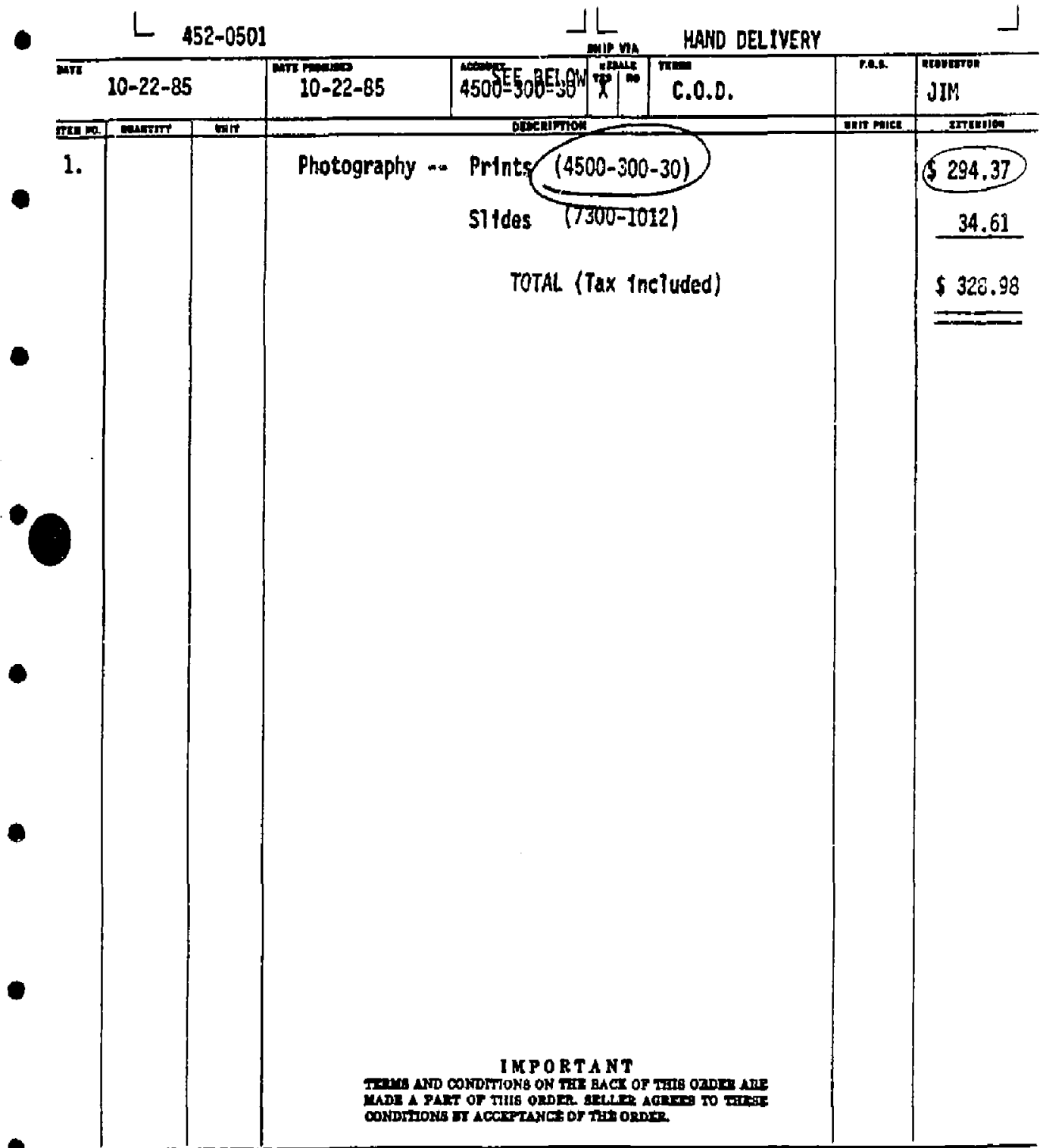

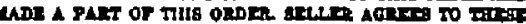

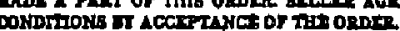

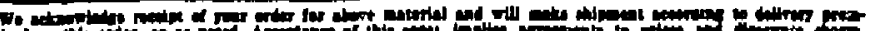

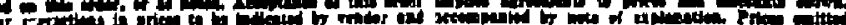

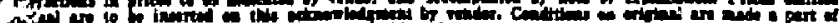

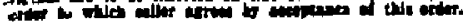

VEDOR BY

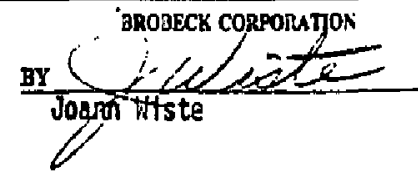

YHITE: Vexdro 
WILLIAM M. BAOBECK \& ASSOCIATES

TL ITEMS PAINTED IN RED MUST

BE FILLED IN BY REQUISITIONER

PURCHASE REQUISITION

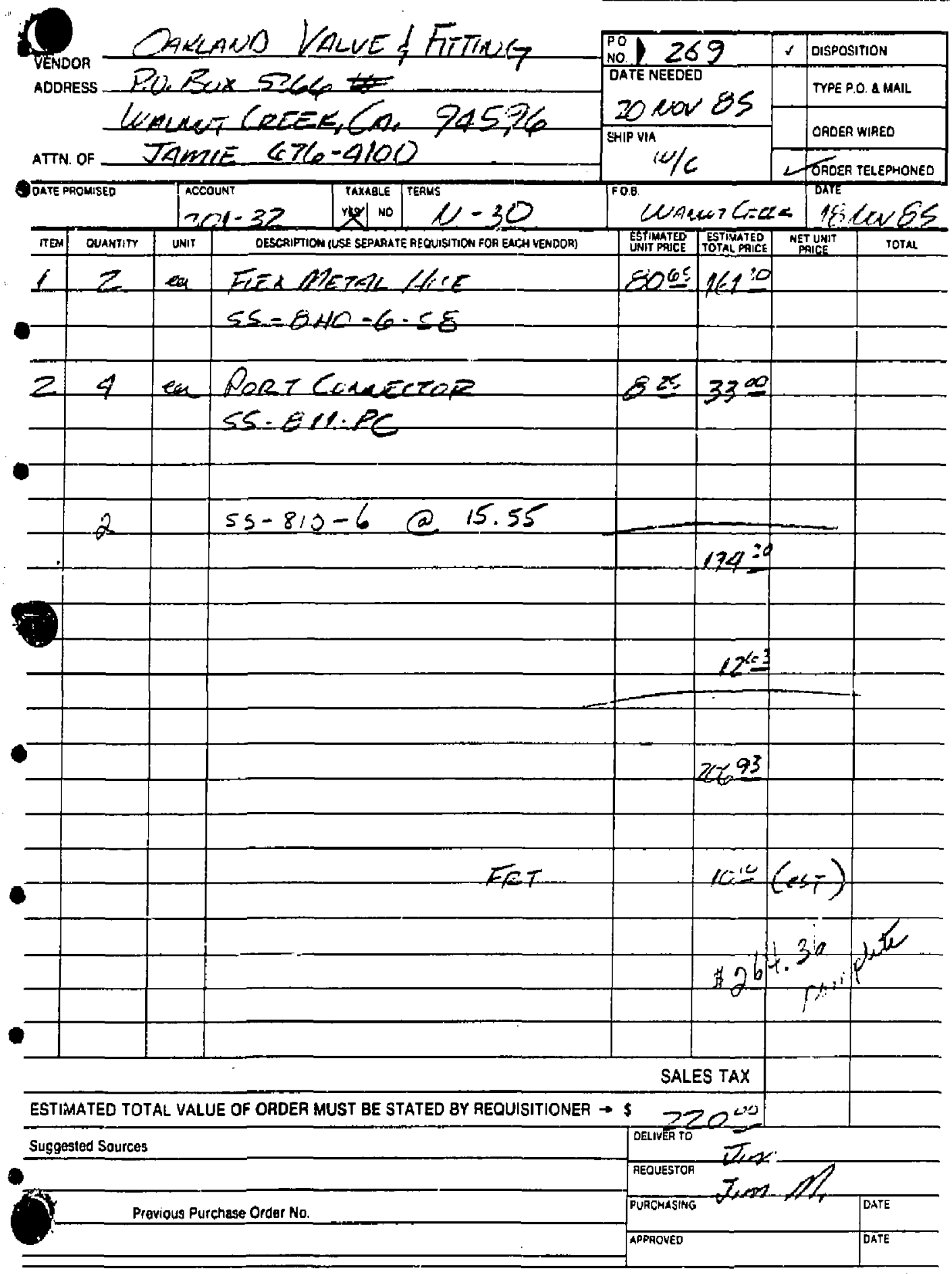

BA.30 (REV 1.79)

ACCOUNTS PAYABLE

C-45 
WILLIAM M. BROBECK \& ASSOCIATES

ALL ITEMS PRINTED IN RED MUST

BE FILLED IN BY REQUISITIONER

PURCHASE REQUISITION

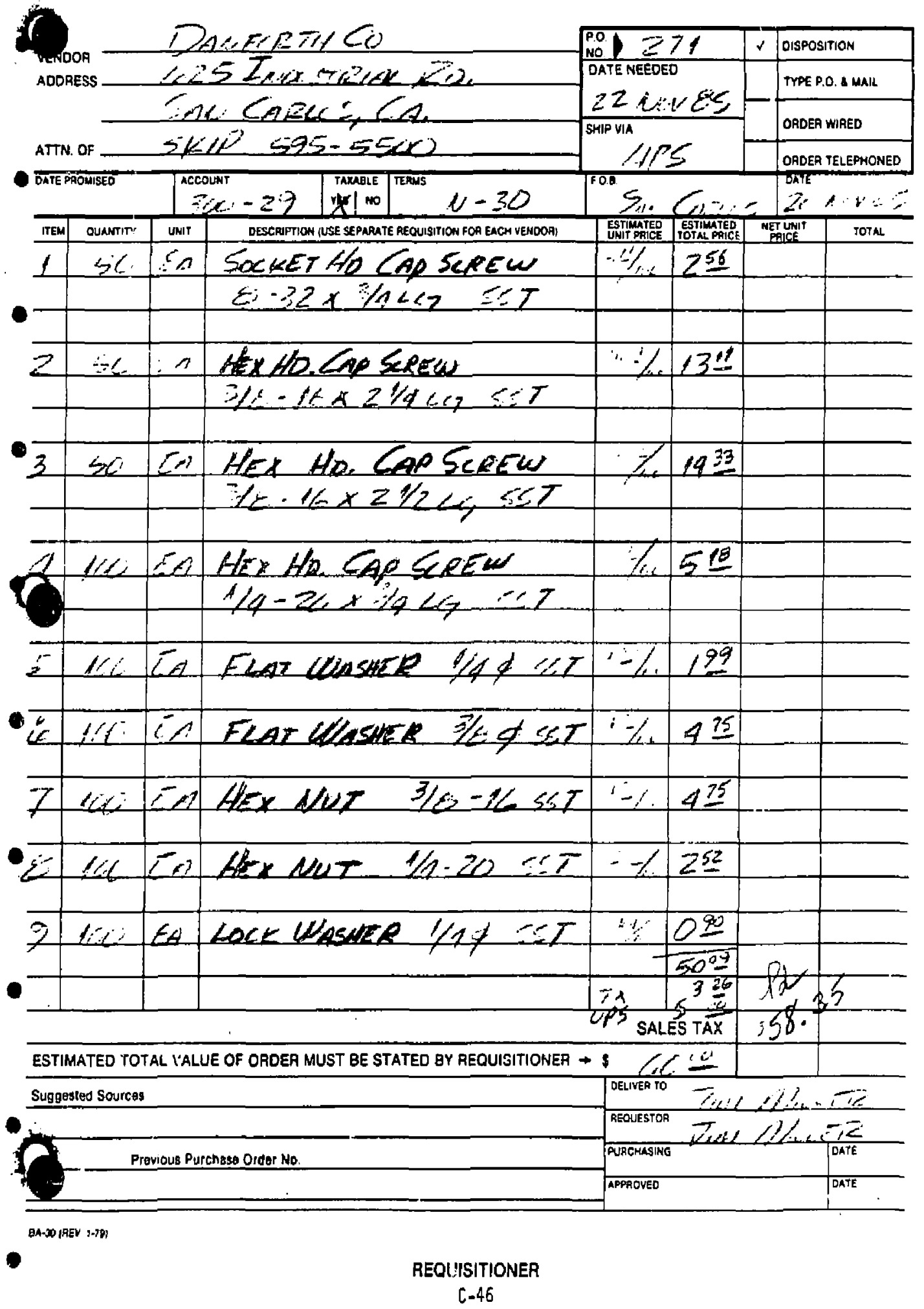


ILL ITEMS PAINTEO IN RED MUST BE FILLED IN BY REOUISITIONER

\section{PURCHASE REQUISITION}
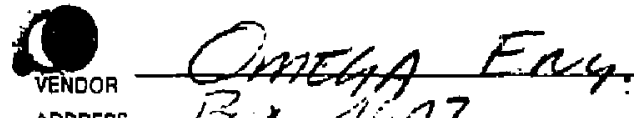

ADDAESS

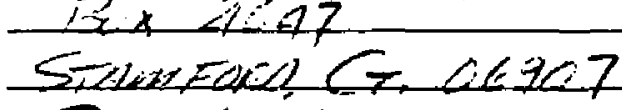

ATTN OF DU (203) 359.1660

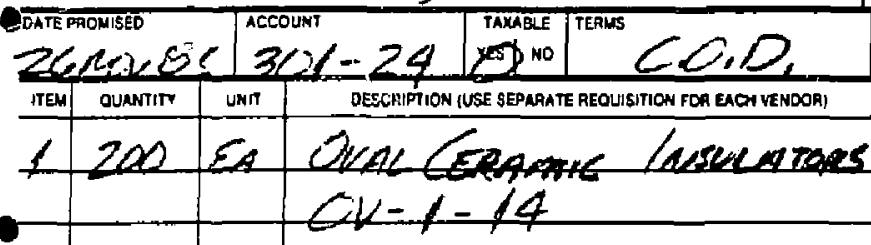

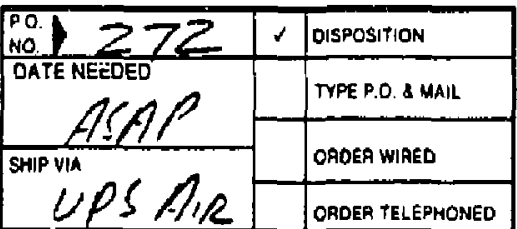

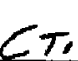

OAIE
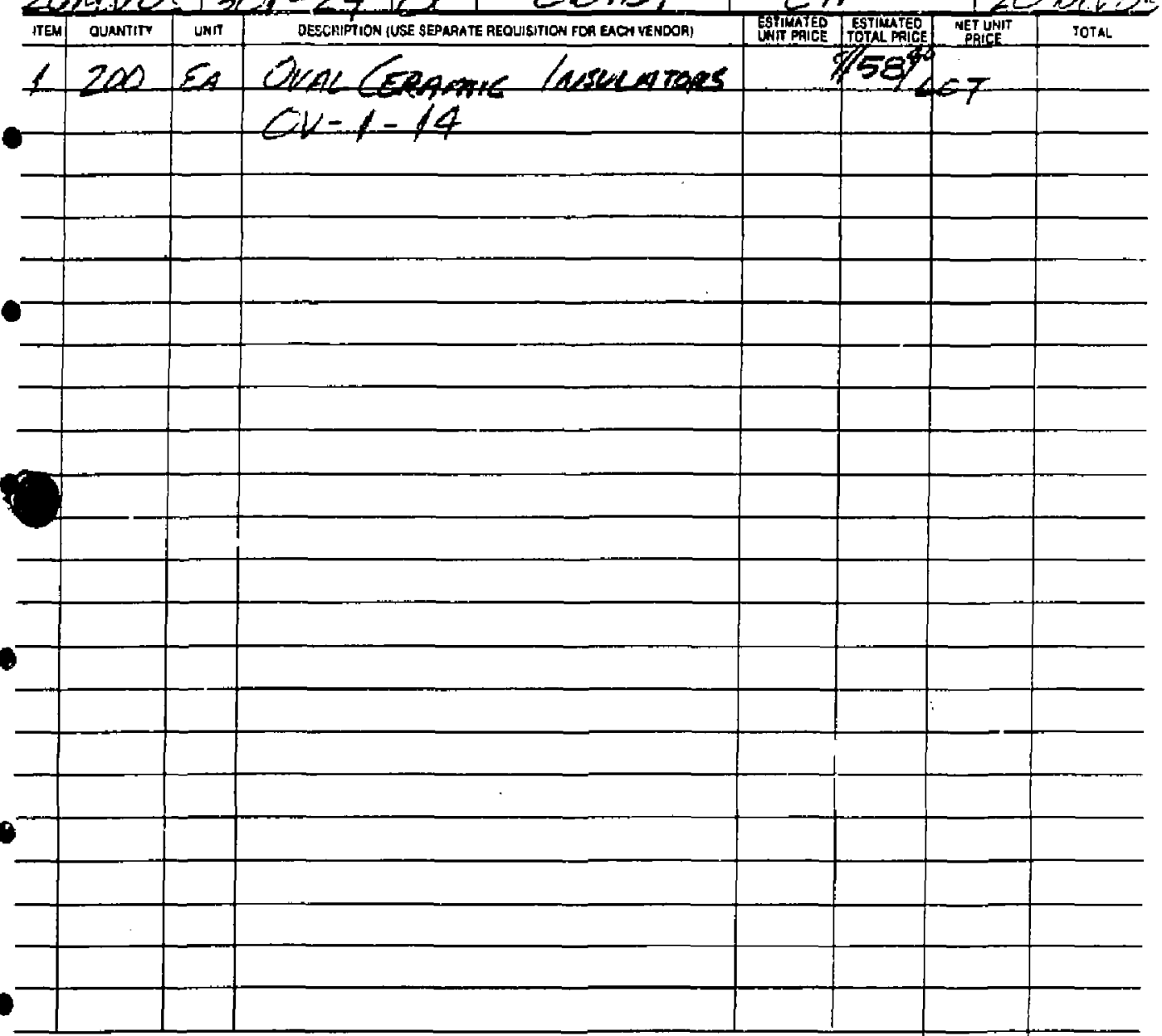

ESTIMATED TOTAL VALUE OF ORDER MUST BE STATED BY REOUISITIONER $\rightarrow$ 
\&LL ITEMS PRINTED IN AEO MUST BE FILLED IN BY REOUISITIONER

PURCHASE REQUISITION

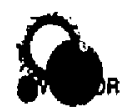

Covac Fryetints

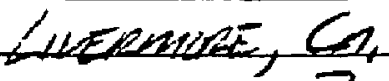

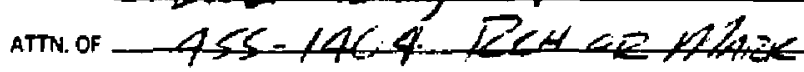

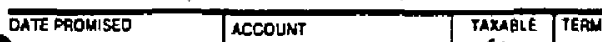

ADDRESS

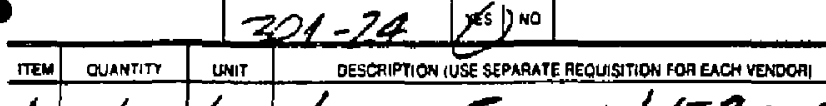

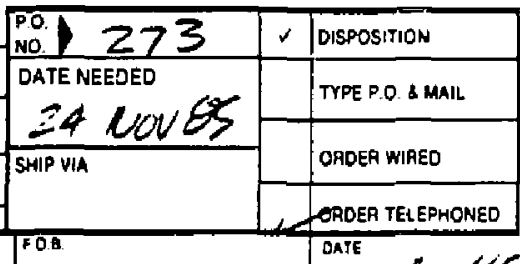

1

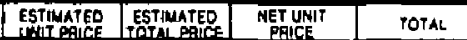

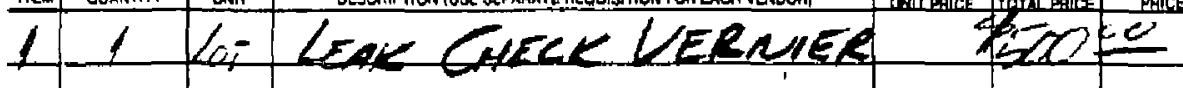

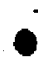


LL ITEMS PAINTED IN RED MUST BE FILLLD IN BY REOUISITIONER

PURCHASE REQUISITION

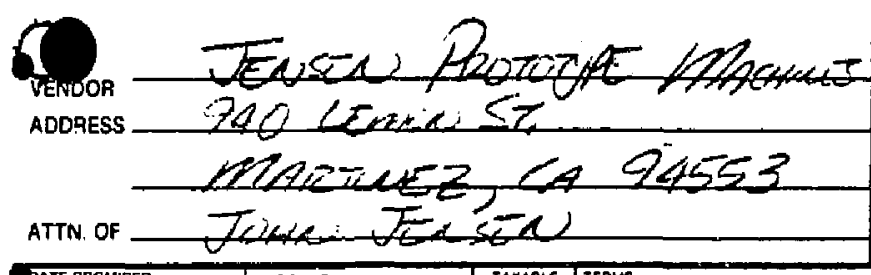

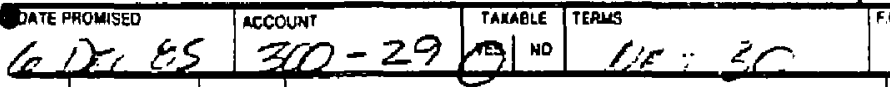

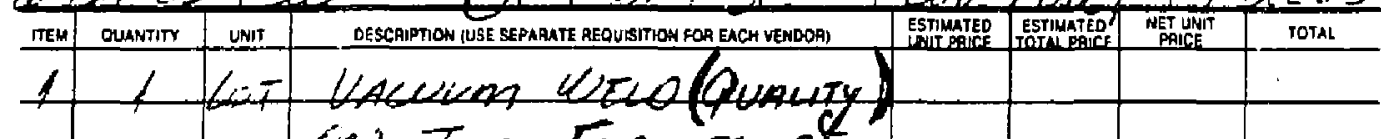

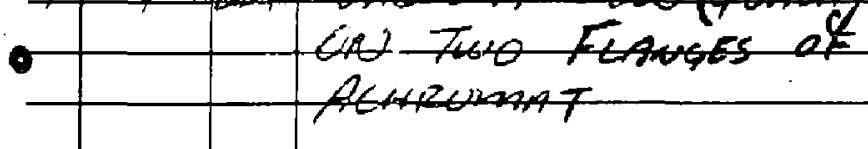

21 WIT AROOHY WATFE

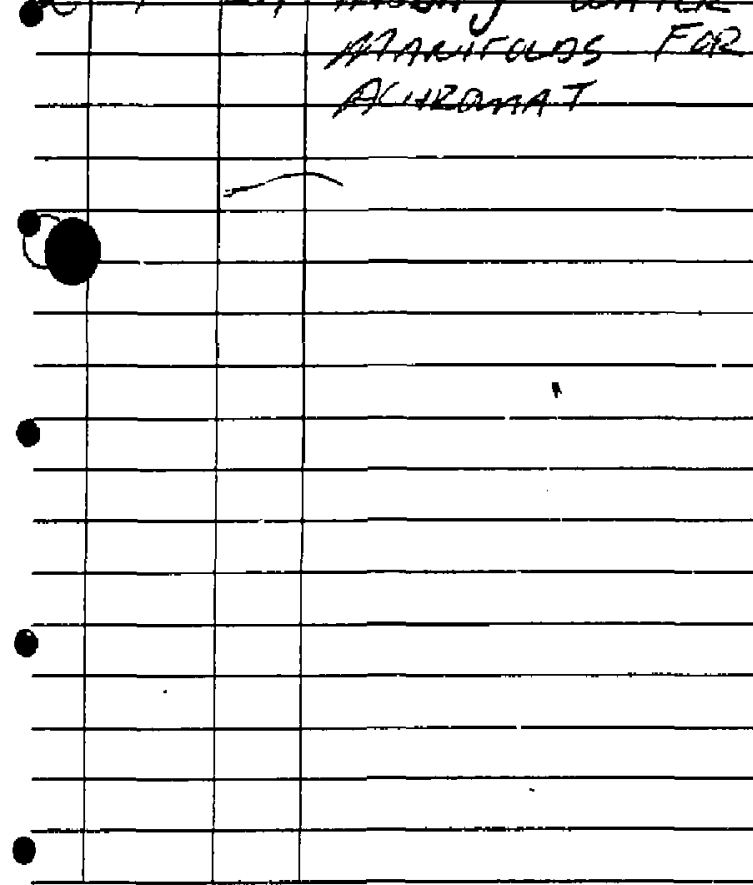

\begin{tabular}{|l|l|l|}
\hline PO. 282 & $\checkmark$ & DISPOSITION \\
\hline DATE NEEDED & & TYPE PO \& MAIL \\
\hline ASAP & & ORDER WIRED \\
\hline SHP VIA & OADER TELEPHONEO \\
\hline
\end{tabular}
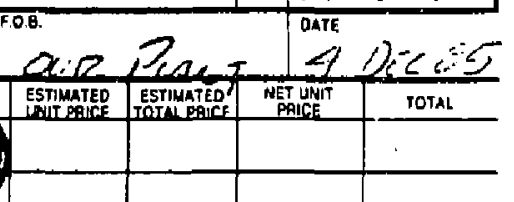

ESTIMATED TOTAL VALUE OF ORDER MUST BE STATED BY REQUISITIONER $\rightarrow$

Suggested Suurces

Pre'uous Purchnse Orage No.

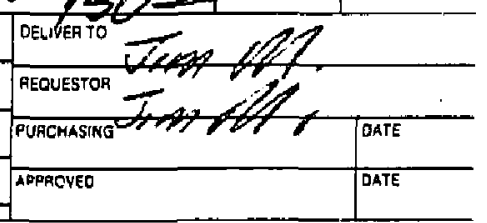


ALL ITEMS PRINTED IN RED MUST

BE FILLEO IN BY REOUISITIONER

PURCHASE REQUISITION

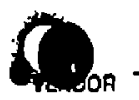

Conac Yacunn Services

ADDAESS P. D. BOX 2531

Livereore, CA 94550

ATTN. OF

DRTE PAOWISEO

$12 / 17 / 85$

$2 \quad{ }^{\text {account }}$

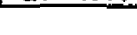

\begin{tabular}{|c|}
\hline 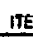 \\
\hline
\end{tabular}

\begin{tabular}{l|l|l|l|l}
1 & 1 & lot & Leak check achromat assenbly .. \\
\hline
\end{tabular}

5 hrs. $\$ 42.50$

\begin{tabular}{|c|c|c|}
\hline \begin{tabular}{l|l} 
P.O. & 292 \\
No. & 292
\end{tabular} & 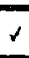 & DISFOSITION \\
\hline DATE NEEDED & & TYPE P.O. \& MAIL \\
\hline $12 / 17 / 85$ & & \\
\hline \multirow{2}{*}{$\begin{array}{l}\text { SHIP VIM } \\
\text { del, }\end{array}$} & & ORDER WIRED \\
\hline & & ORDEA TELEPHONEO \\
\hline F.0.6 & & $\begin{array}{l}\text { OATE } \\
12 / 17 / 85\end{array}$ \\
\hline
\end{tabular}

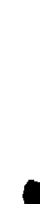


A complete set of as-built drawirgs for the achromat is included in this appendix.

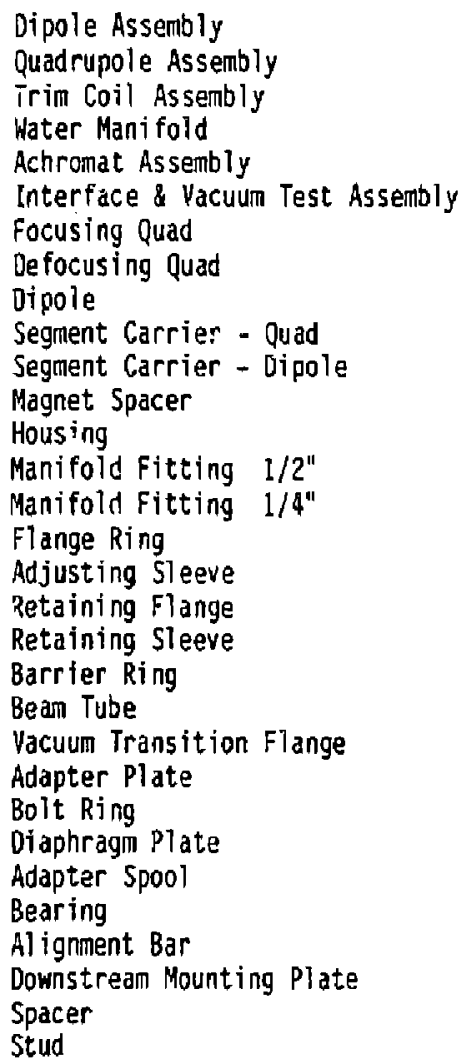

9501751

95(1750

9501740

$95 \mathrm{C} 1743$

9511749

9501781

9501732

9501733

9501734

$95 C 1736$

$95 C 1737$

9581738

95.1739

9581741

9581742

95C 1744

95,1745

9581745

9581747

9581748

9561752

9501774

$95 C 1775$

95C1776

9581777

$95 \mathrm{~B} 1778$

9581779

$95 \mathrm{~B} 1780$

$95 B 1782$

$95 B 1783$

95B1784 

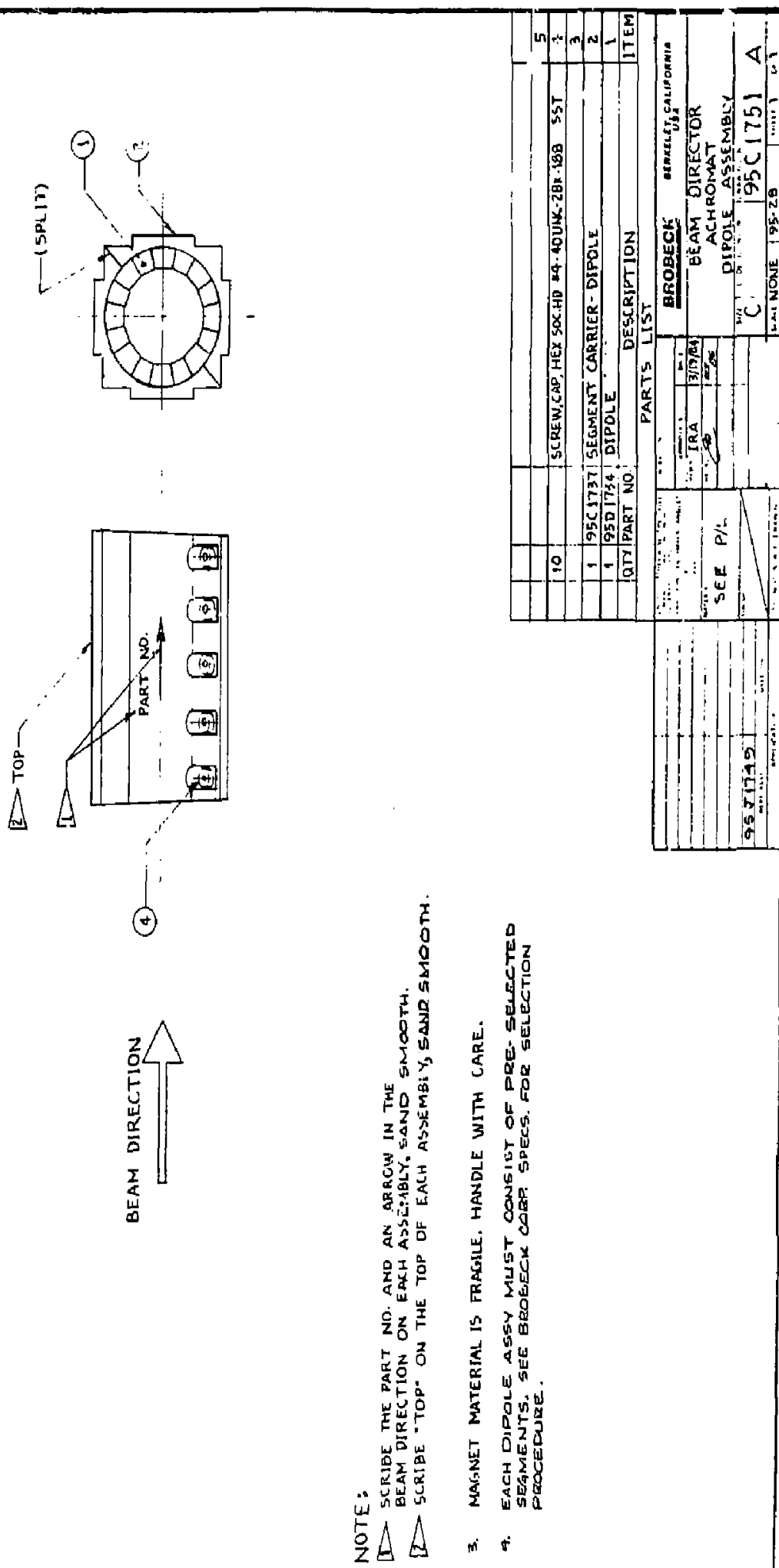

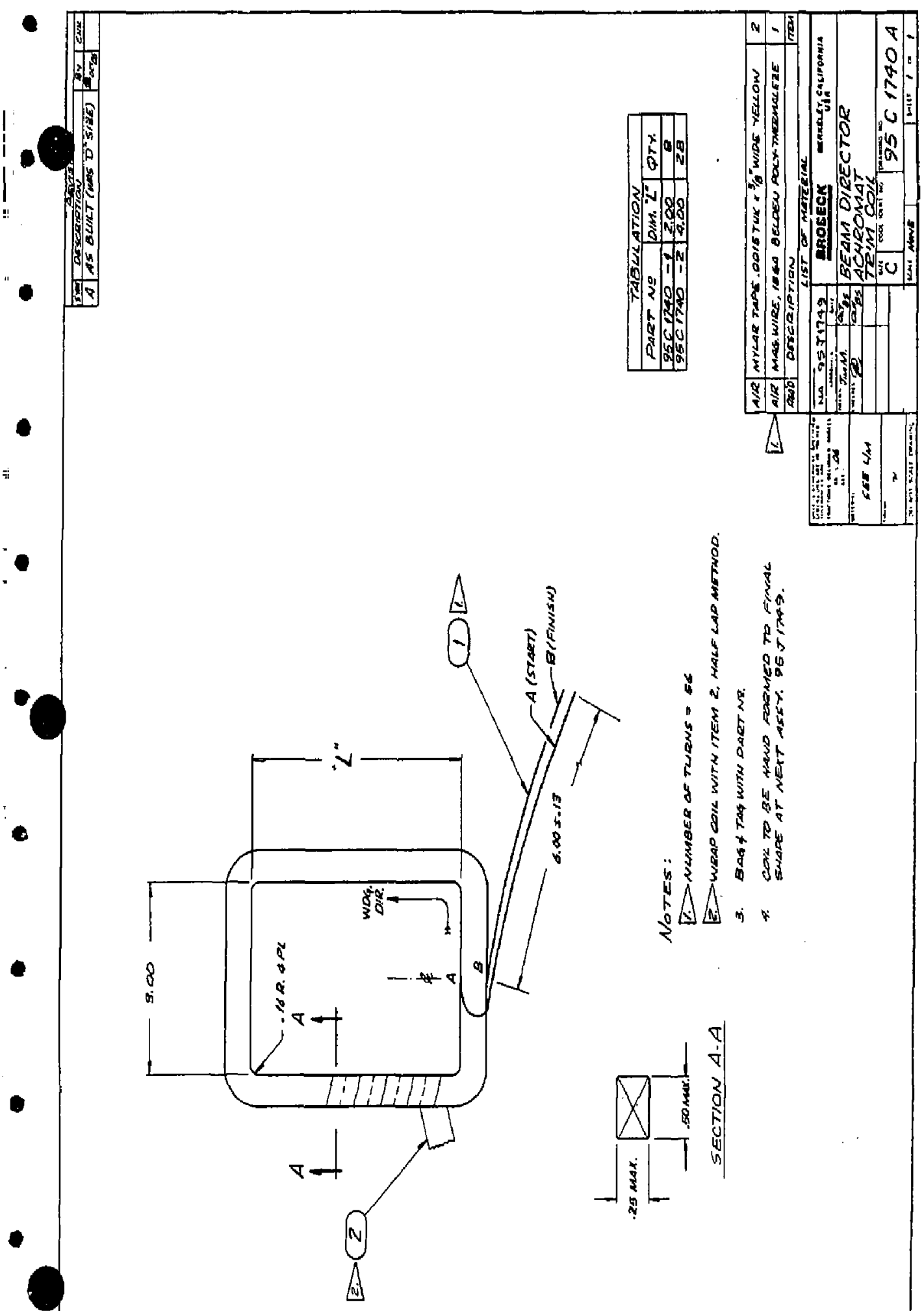

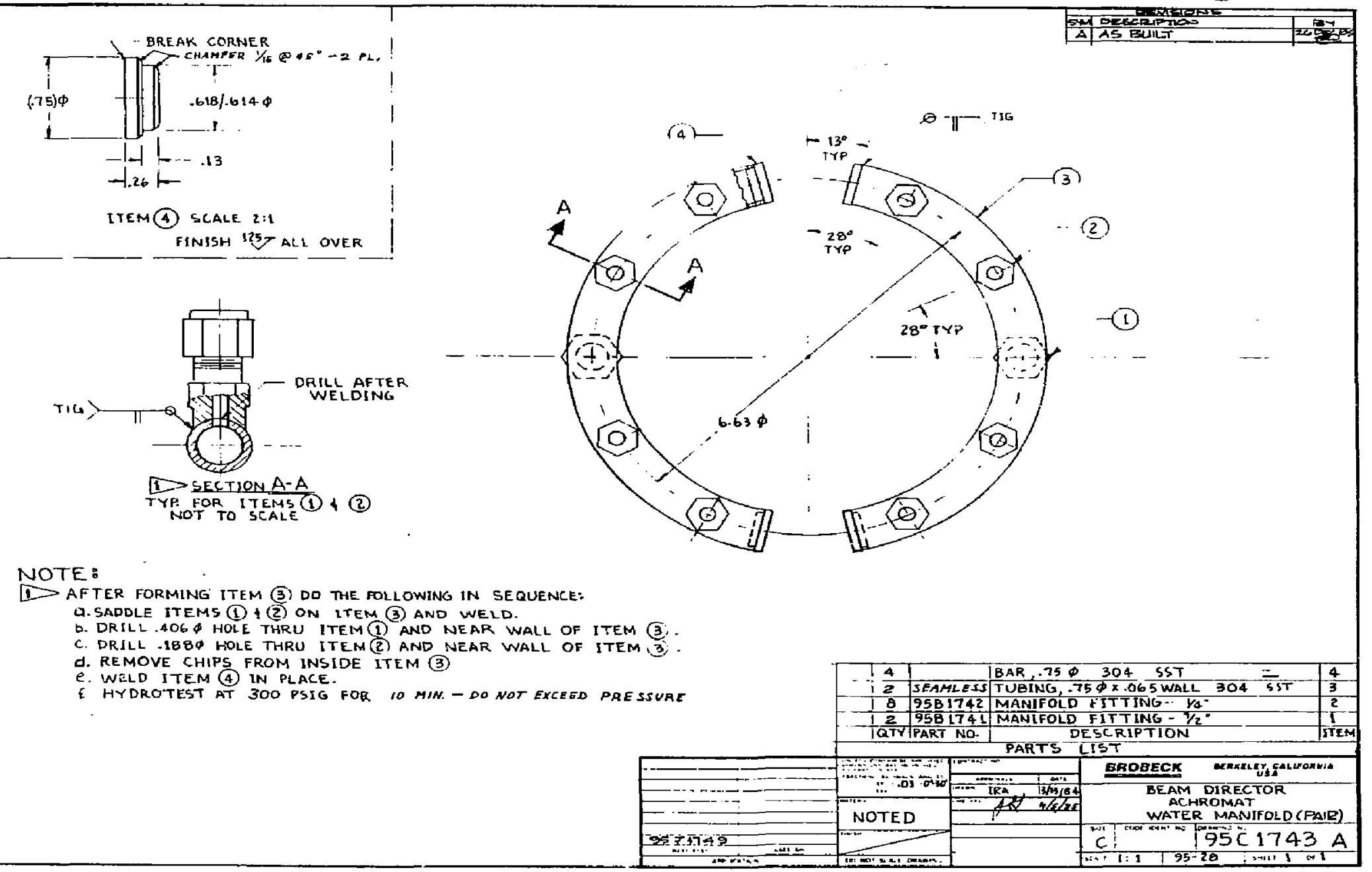

NOTE:

(1) AFTER FORMING ITEM (3) DO THE FOLLOWING IN SEQUENCE:

a. SADDLE ITEMS (1) I (2) ON TTEM (3) AND WELD.

b. DRILL .4OG $\$$ HOLE THRU ITEM(1) AND NEAR WALL OF ITEM (3.

c. DRILL 188 Q HOLE THRU ITEM (2) AND NEAR WALL OF ITEM 3

d. REMOVE CHIPS FROM INSIDE ITEM (3)

e. WELD ITEM (4) IN PLACE.
E HYDROTEST AT 300 PSIG

IO MIN. - DO NOT EXCEED PRESSUAE

\begin{tabular}{|c|c|c|c|c|}
\hline & & & EROBECK & ecexregy feturonts \\
\hline-1 & 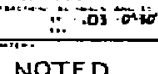 & 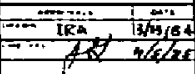 & $\begin{array}{l}\text { BEAM } \\
\text { AC } \\
\text { WATE }\end{array}$ & $\begin{array}{l}\text { DIRECTOA } \\
\text { ROMAAT } \\
\text { MANIFOLD (FAIR) }\end{array}$ \\
\hline 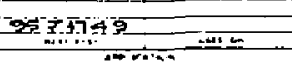 & 1 & & $\frac{c}{1: 1}$ & $95 \div 1743 \mathrm{~A}$ \\
\hline
\end{tabular}




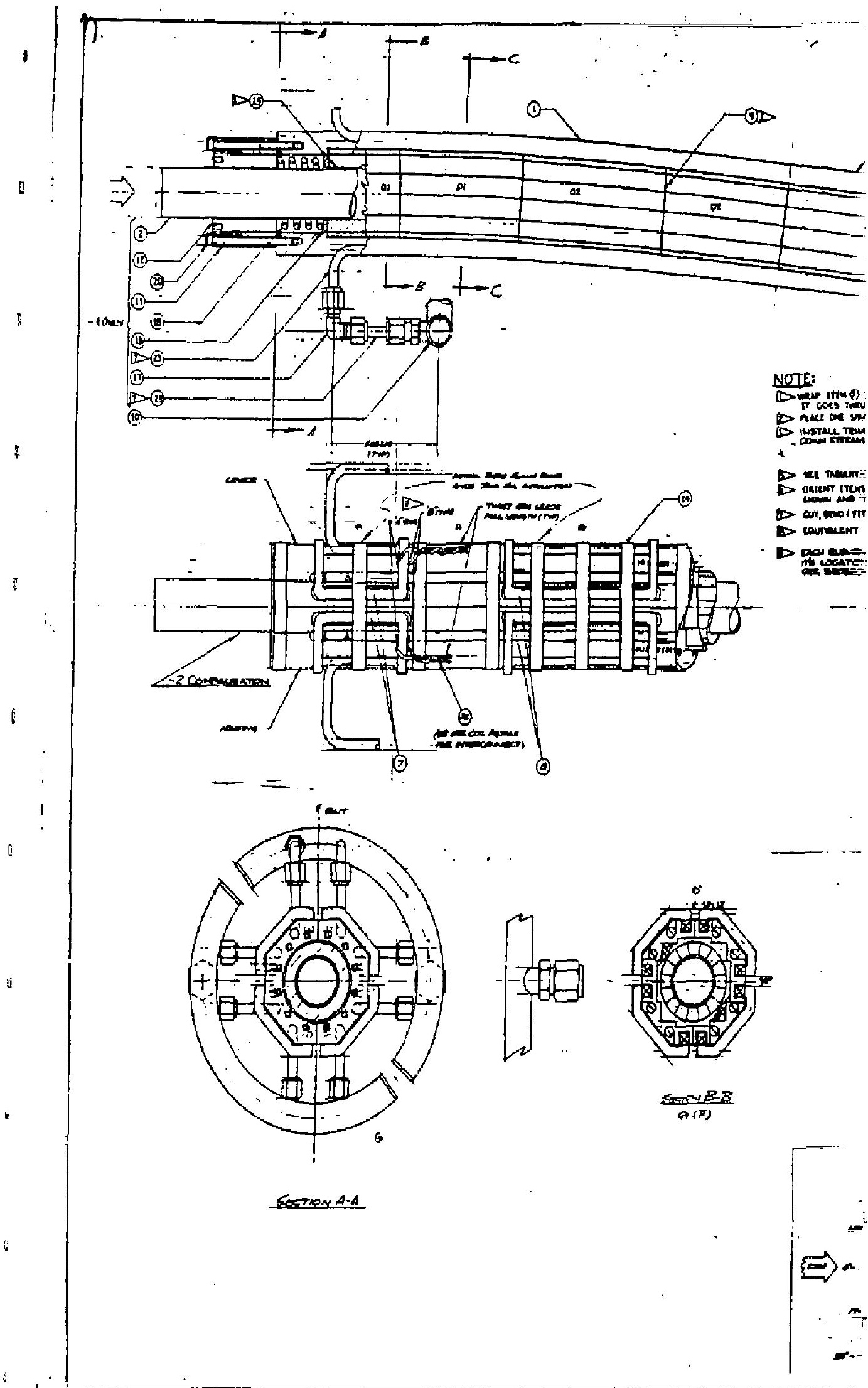




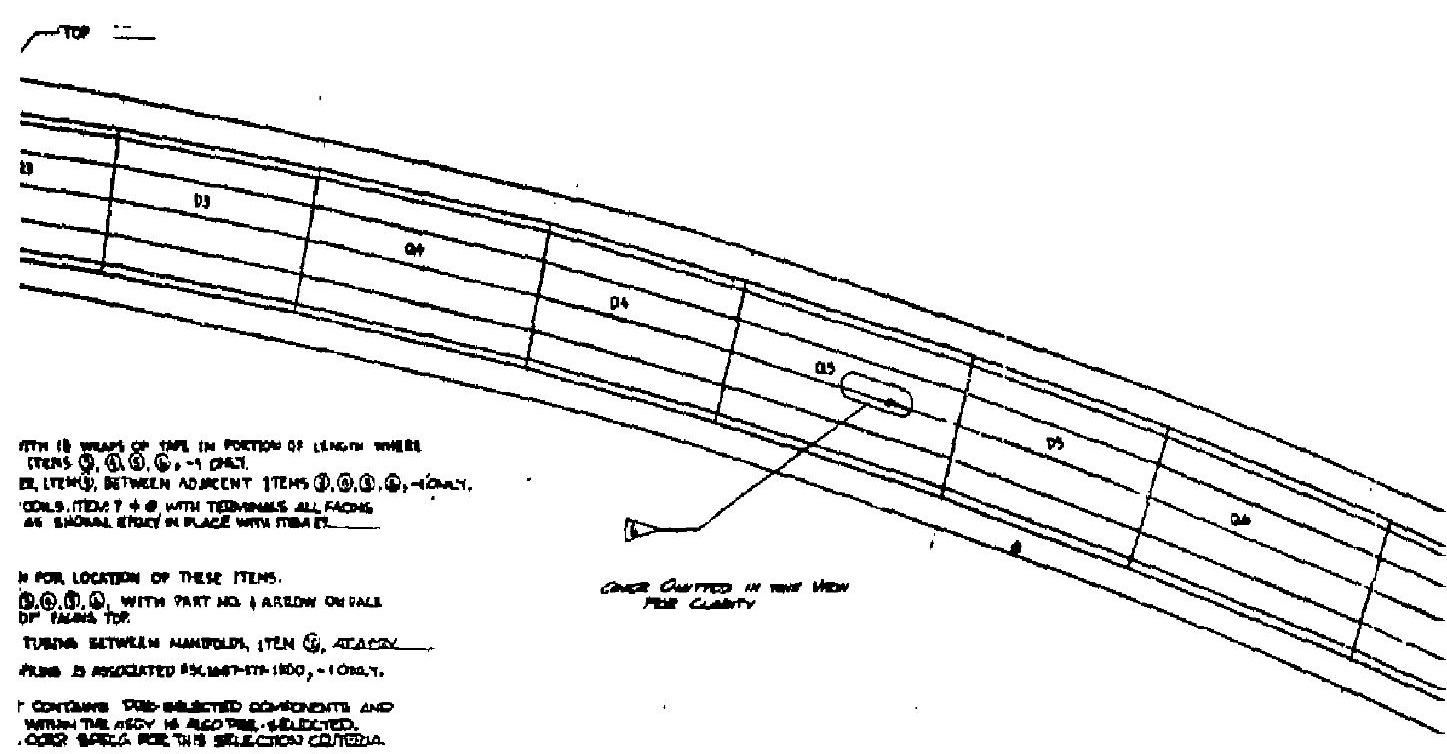

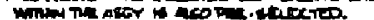
on that in

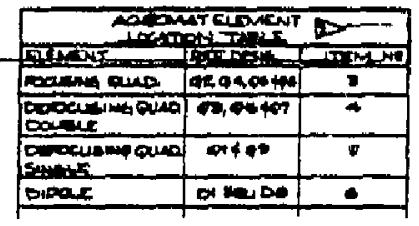

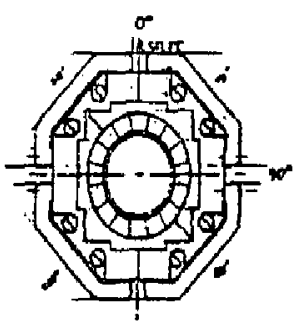

Stran: $E \leq C$

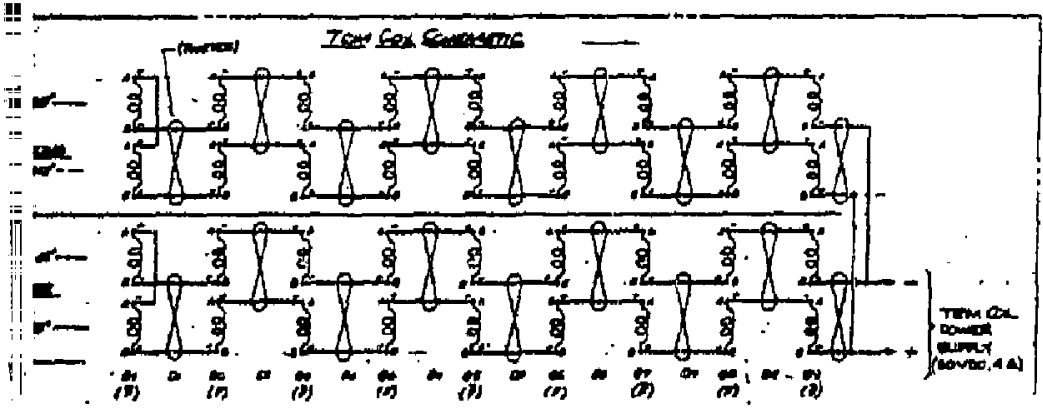




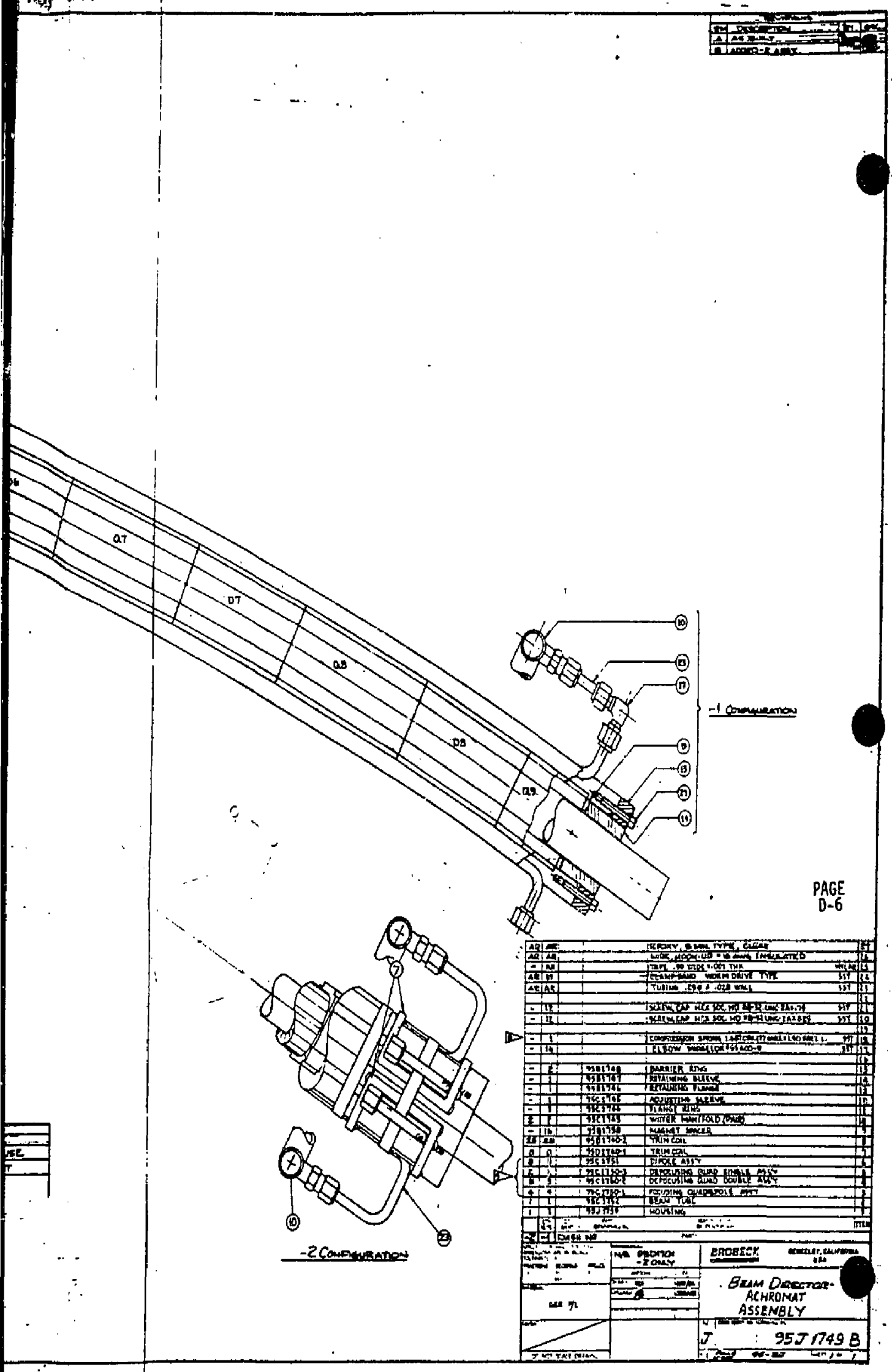



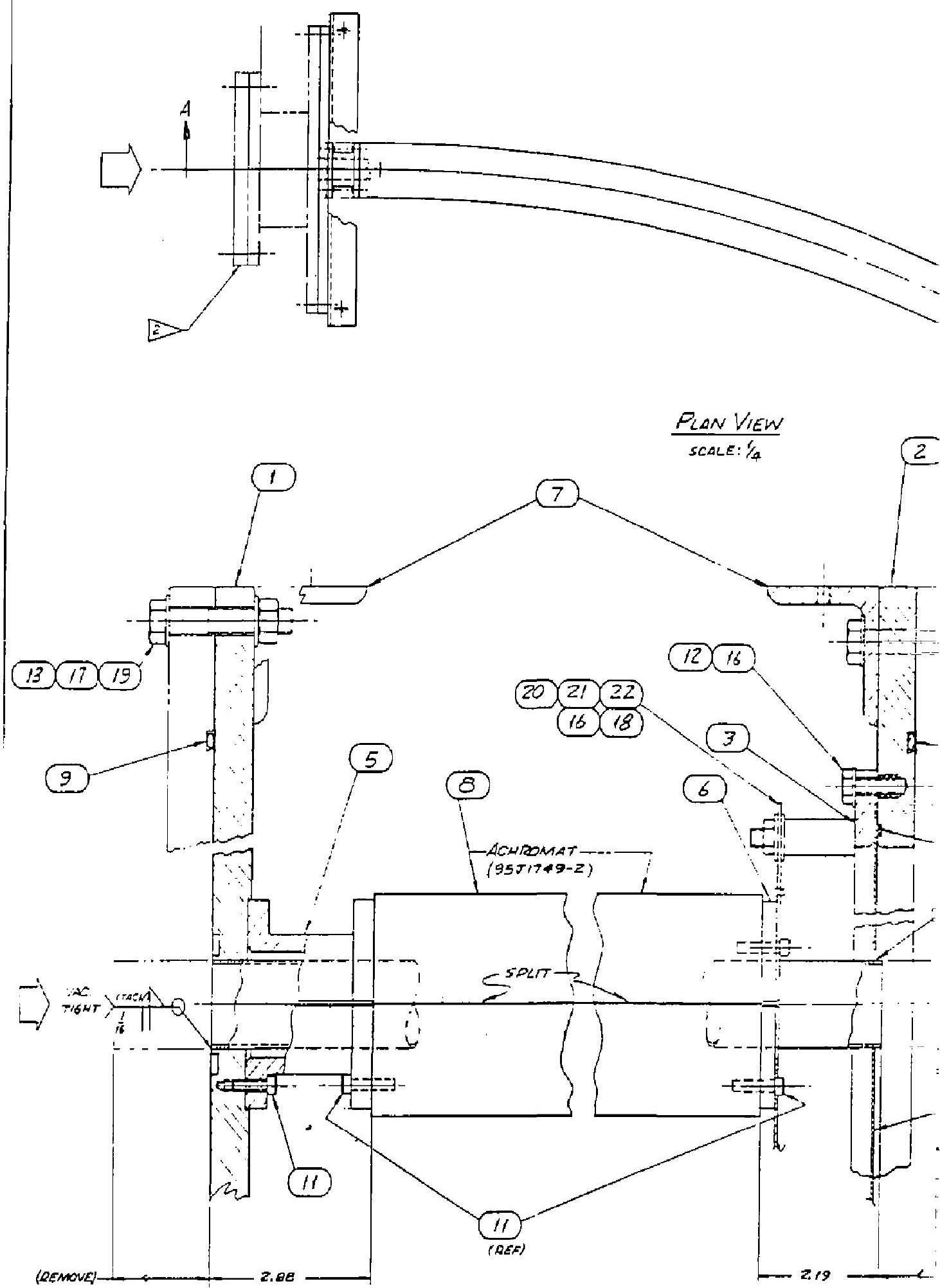


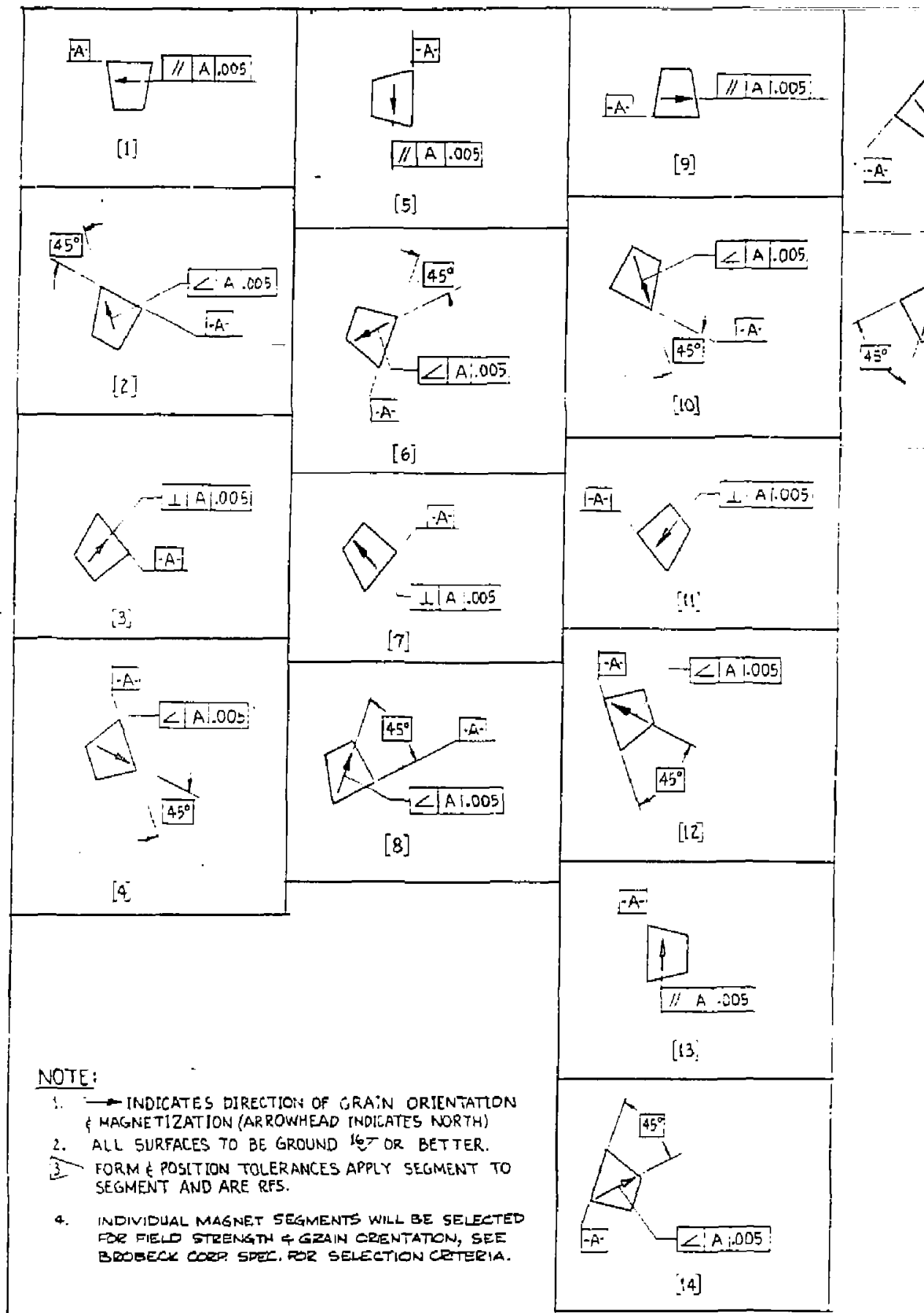



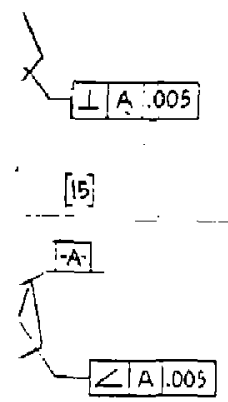

[16]

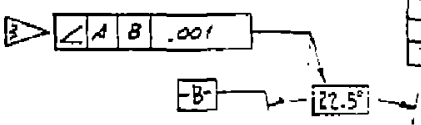

1

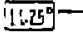

DETAIL A

TYPICAL SEGMENT

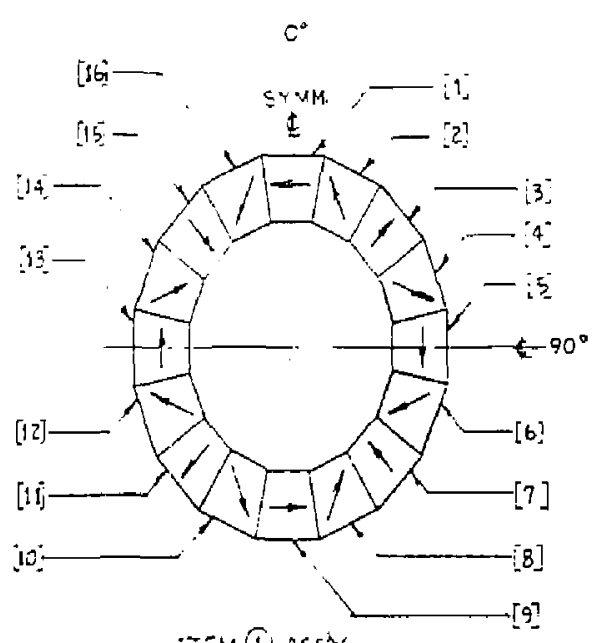

ZEMII: ASSY
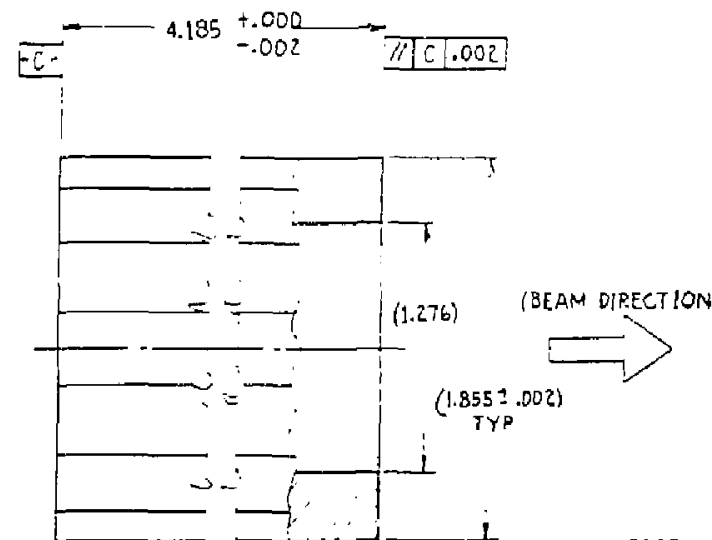

PAGE

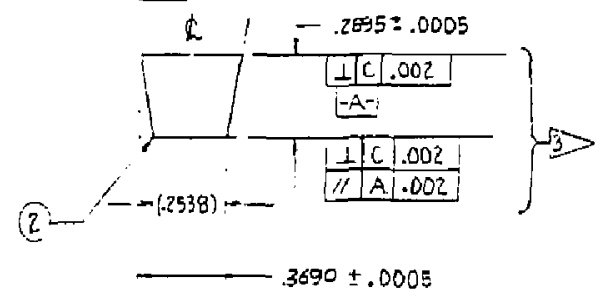



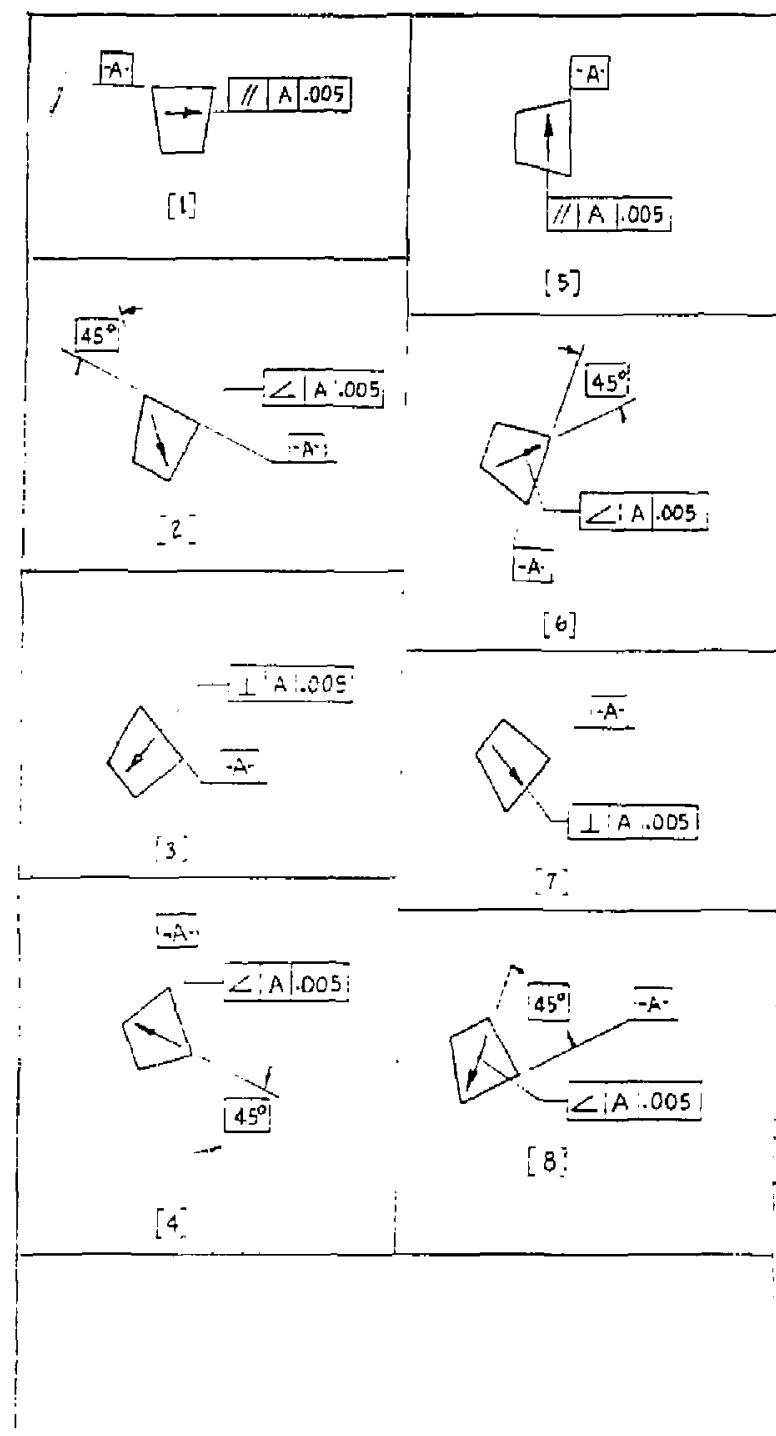

NOTE:

1 - INDICATES DIRECTION OF GRAIN ORIENTATLON MAGNETIZATION (ARROWHEAD INDICATES NORTH) 2 ALL SURFACES TO BE GROUND IGS OR BETTER. D FOR $M$ \&OSITION TOLERANKES APPLY SEGMENT TO SEGMENT AND ARE RFS.

4. INDIVIDUAL MAGNET SEGMENTS WILL BE SELECTED ROR FELD GTRENGTH \& GRAIN ORIENTATION, SEE BROBESK COXP. SPEC, FOR SELECTION CEIERIA.

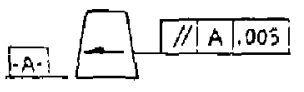

$\left[s^{-}\right.$
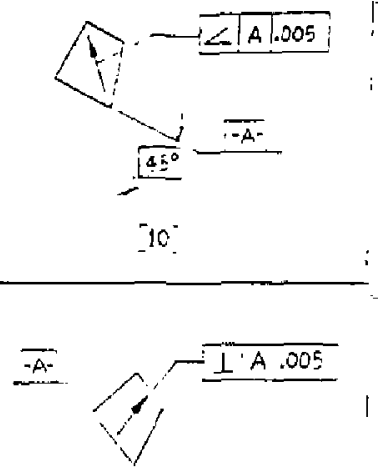

11.

$\overline{-A}-\overline{=A .005}$

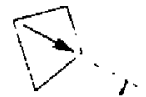

$\overline{45^{2}}$

[12]

-A-

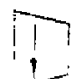

㕕家的

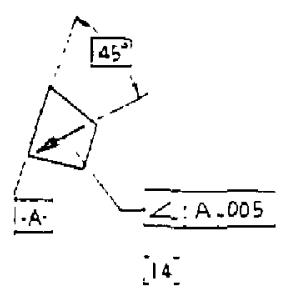




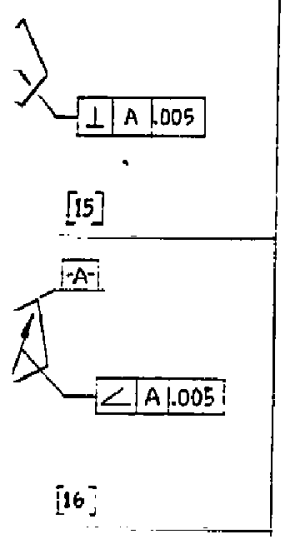

taxis:
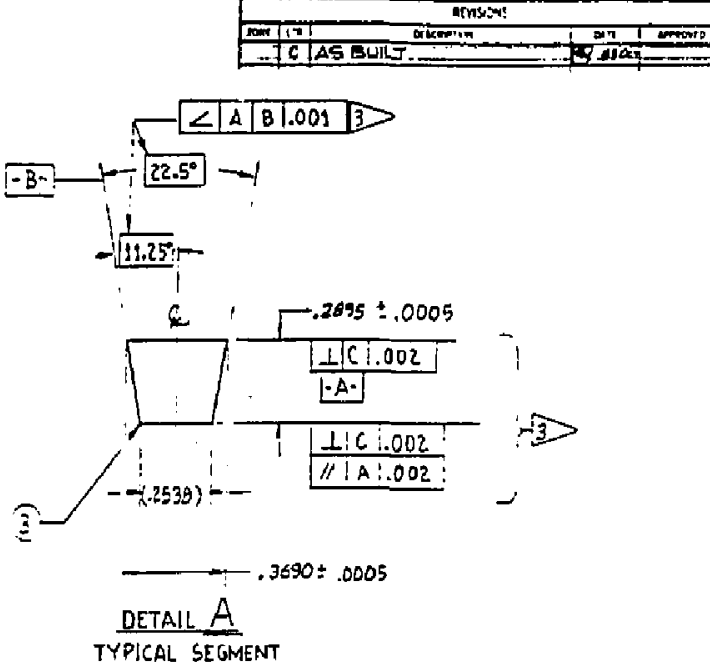

116

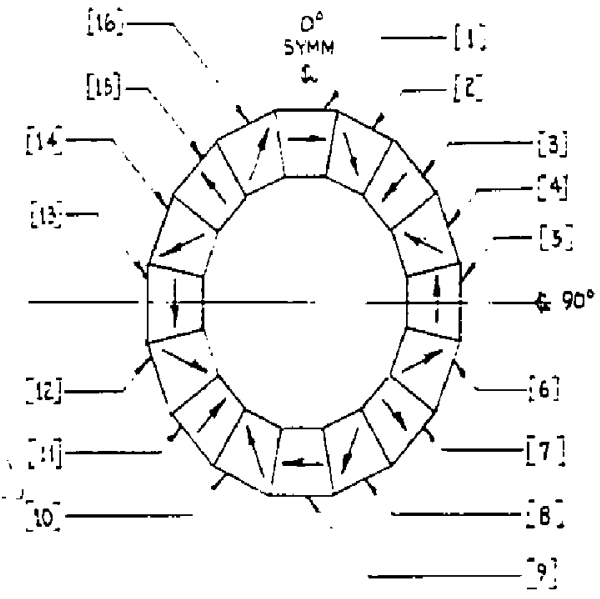

$=\cdots+x_{-.002}^{+.000}-\frac{}{11+.002}$

ITEN OR ITEM (2) ASSEMBLY

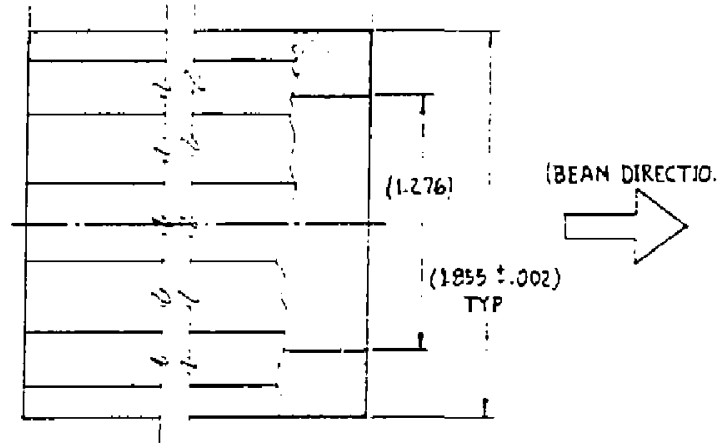

\begin{tabular}{|l|}
\hline -A3U:ATION \\
\hline PART NO I DIM X \\
\hline 95[1733-11 4.185 \\
\hline 95D 1733-2! 2.093 \\
\hline
\end{tabular}

PAEE
$0-9$

OREENTED GRAIN FERRTL PERM, MAGNET MAT'L' 3

$1 0 \longdiv { 1 0 }$ $.950173^{3}-11$ DEFOCUSING QUAQ DOUBLE-ASS'Y DESLRIPTION OTYZTT FART NR.

$-2 \mid-1$-DASH WA PARTS LIST

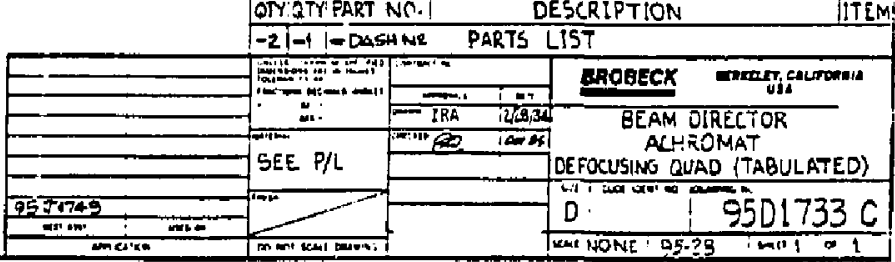




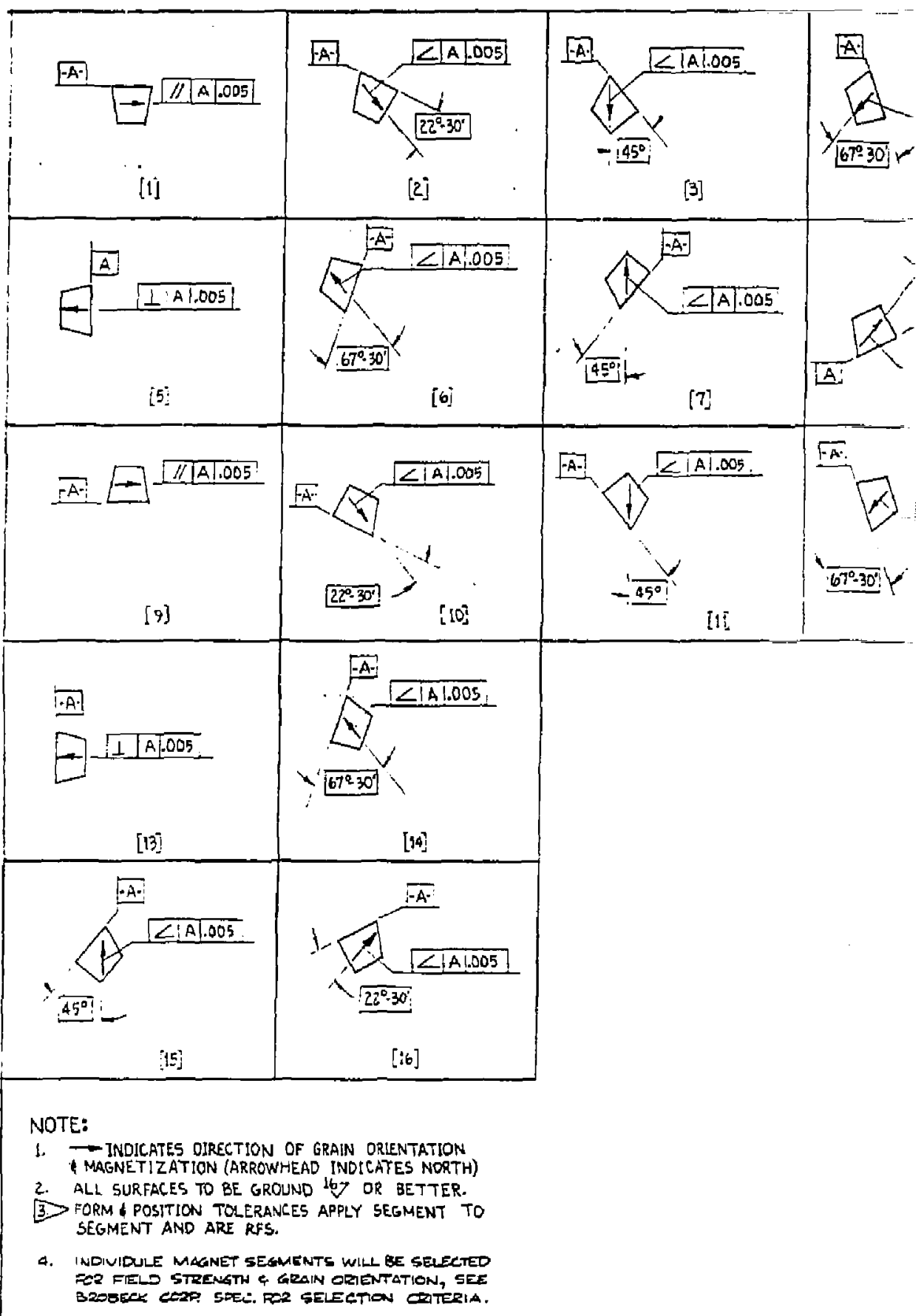




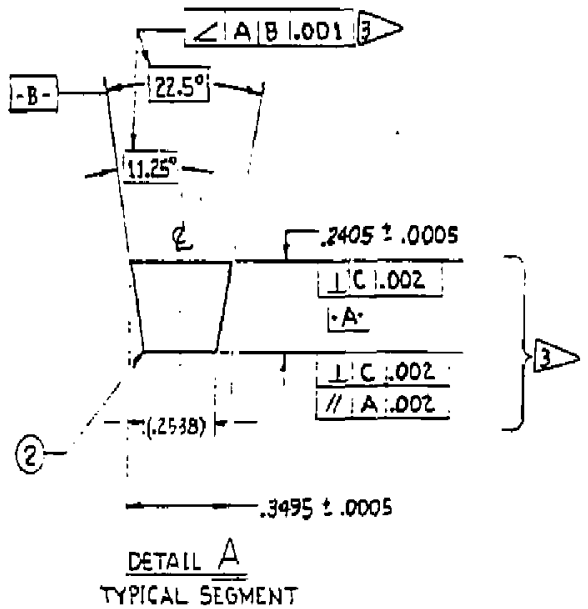

i8

TYPICAL SEGMENT

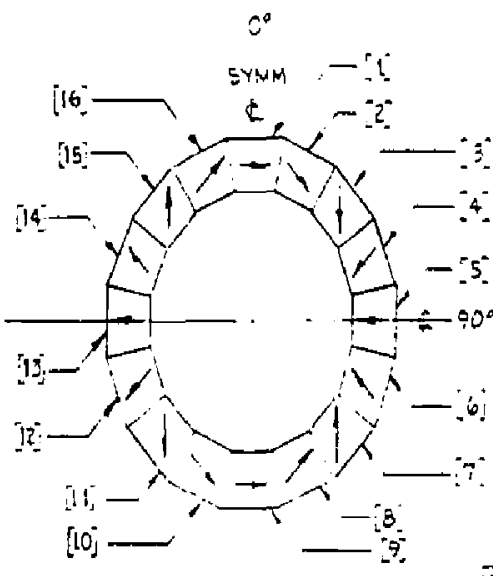

ITEM (1; ASSEIABLY

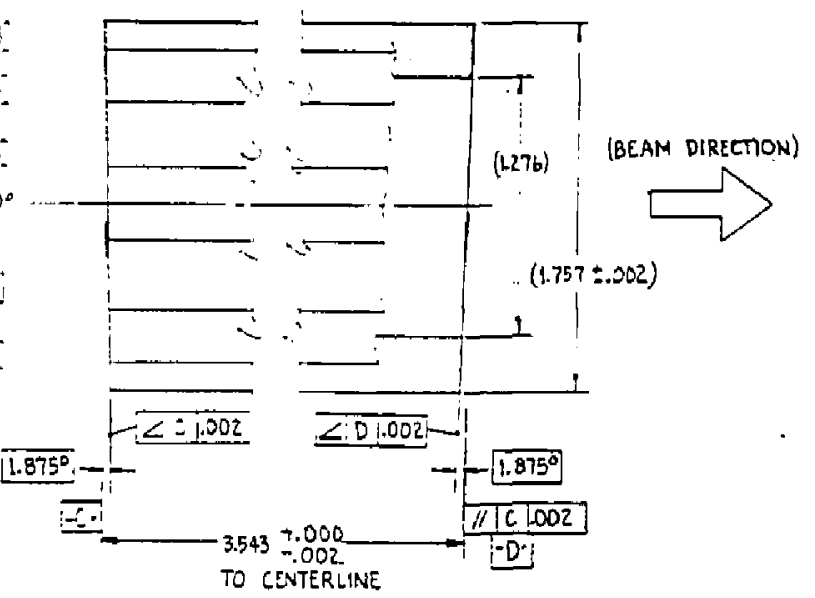

PAGE

D-10

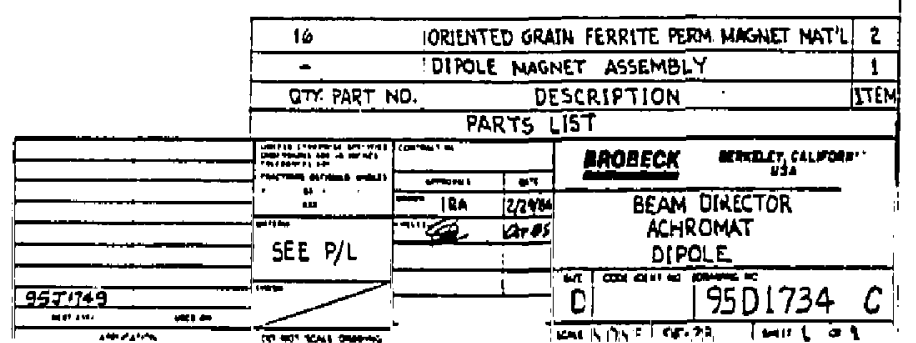




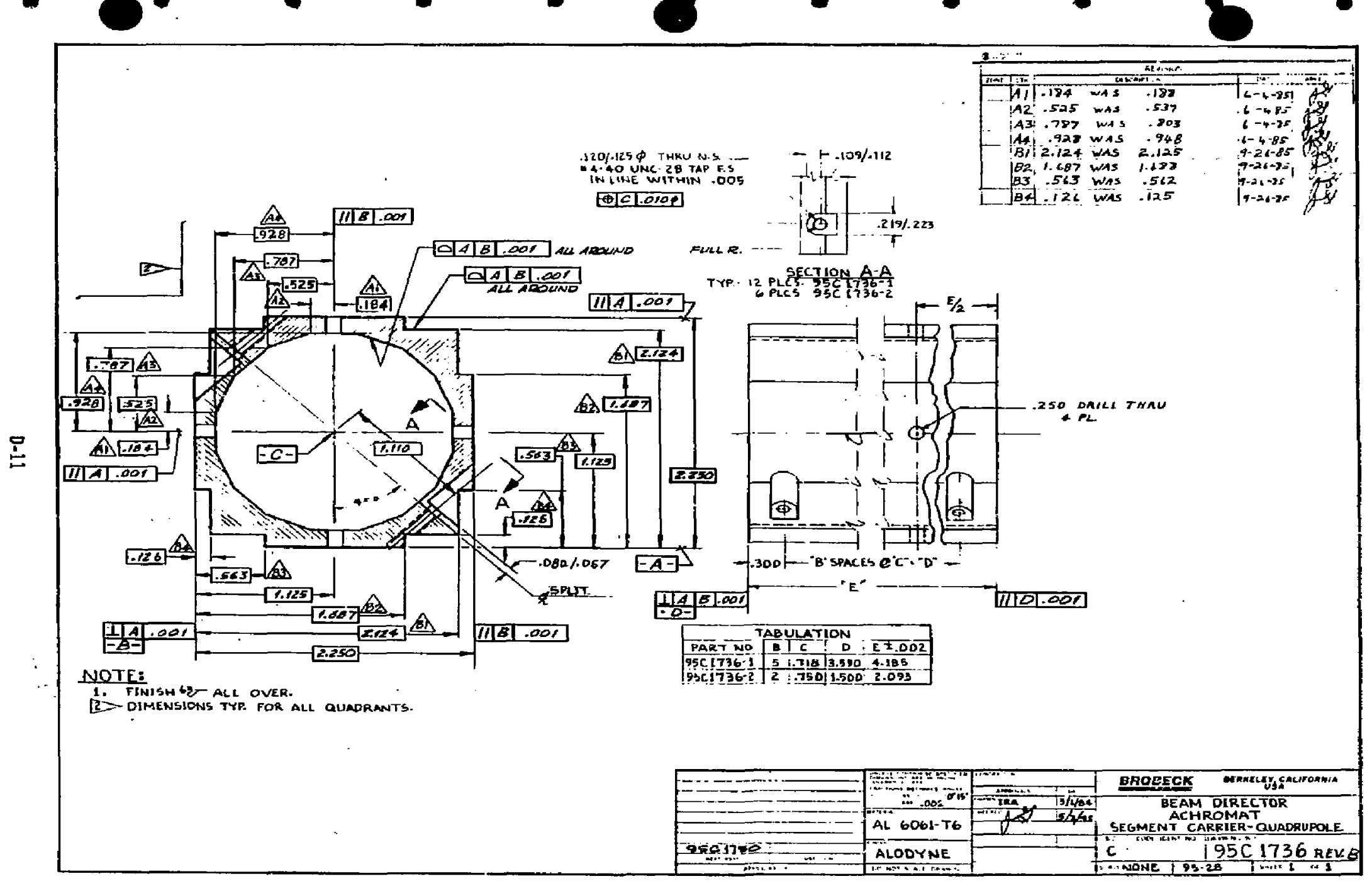




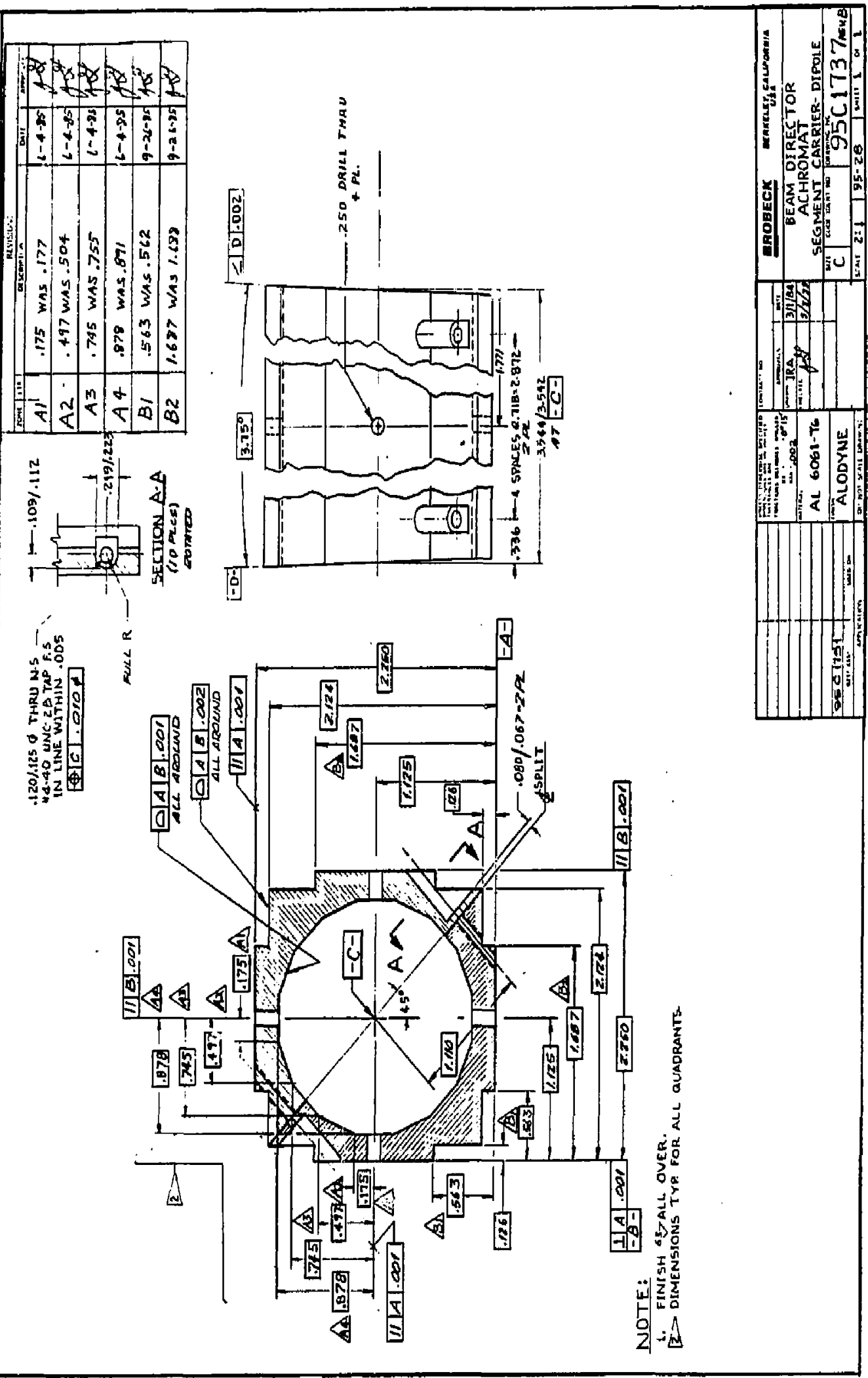




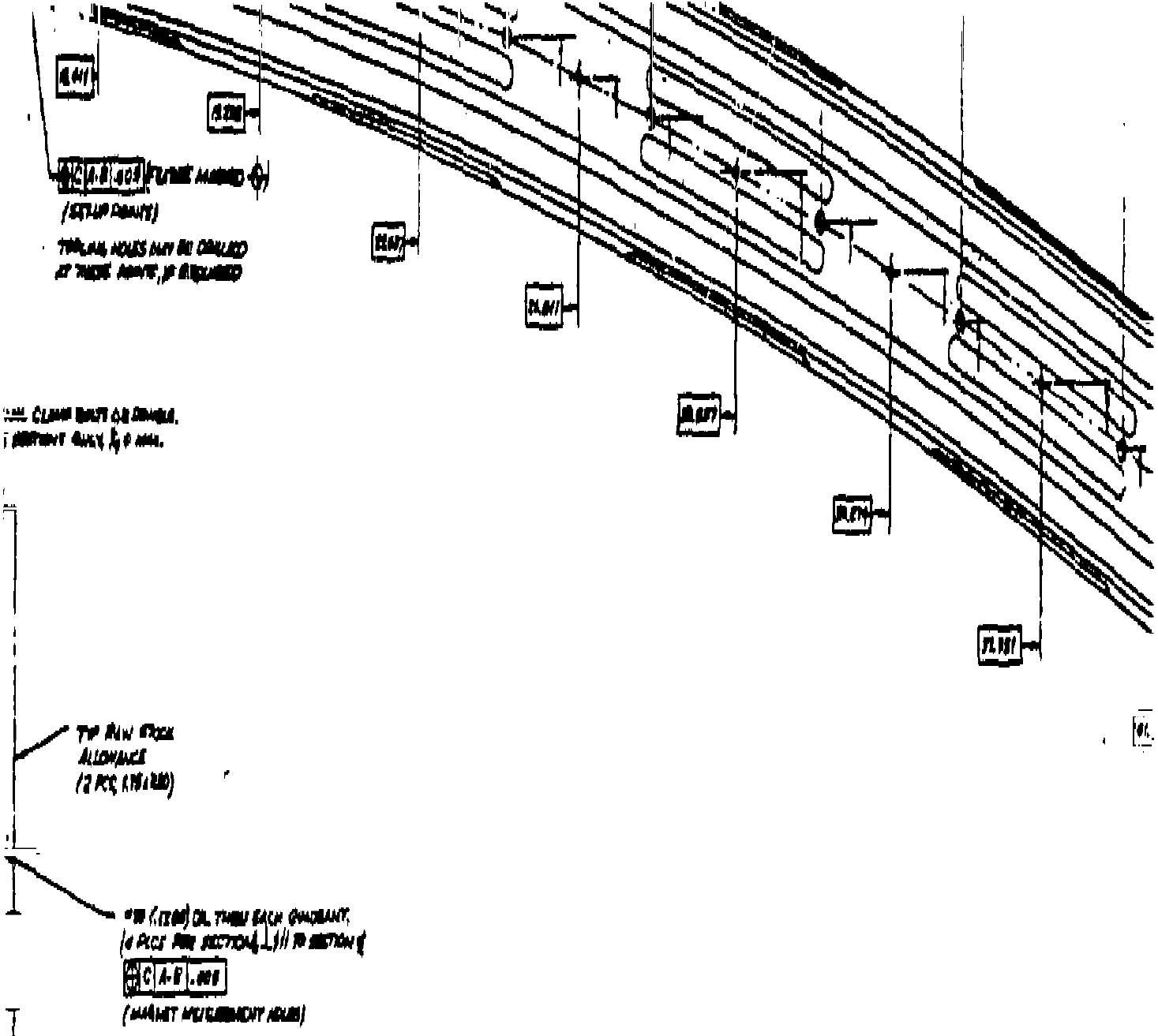

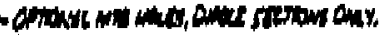
$y+w$

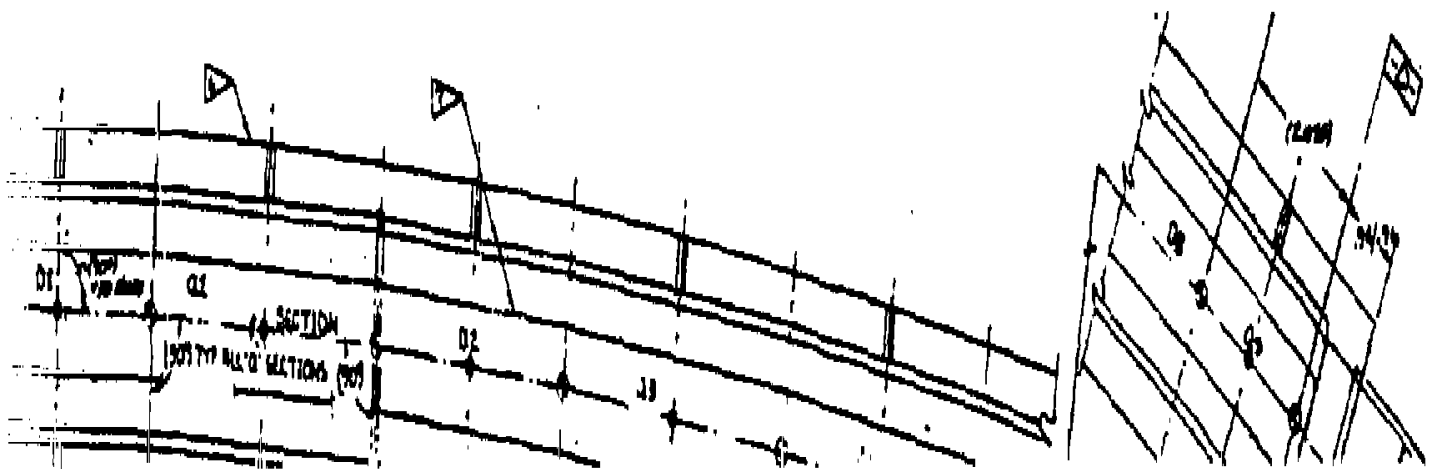




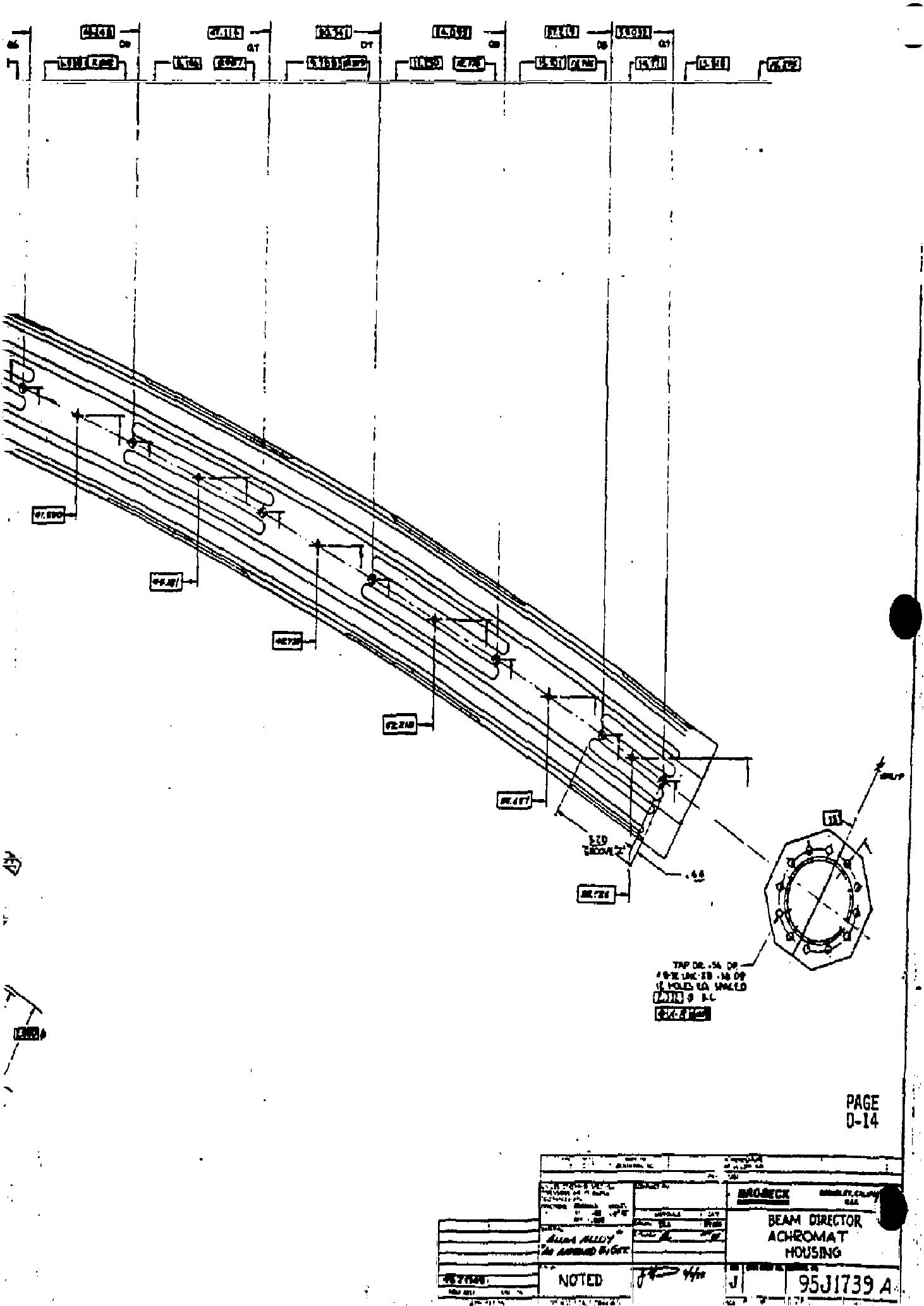



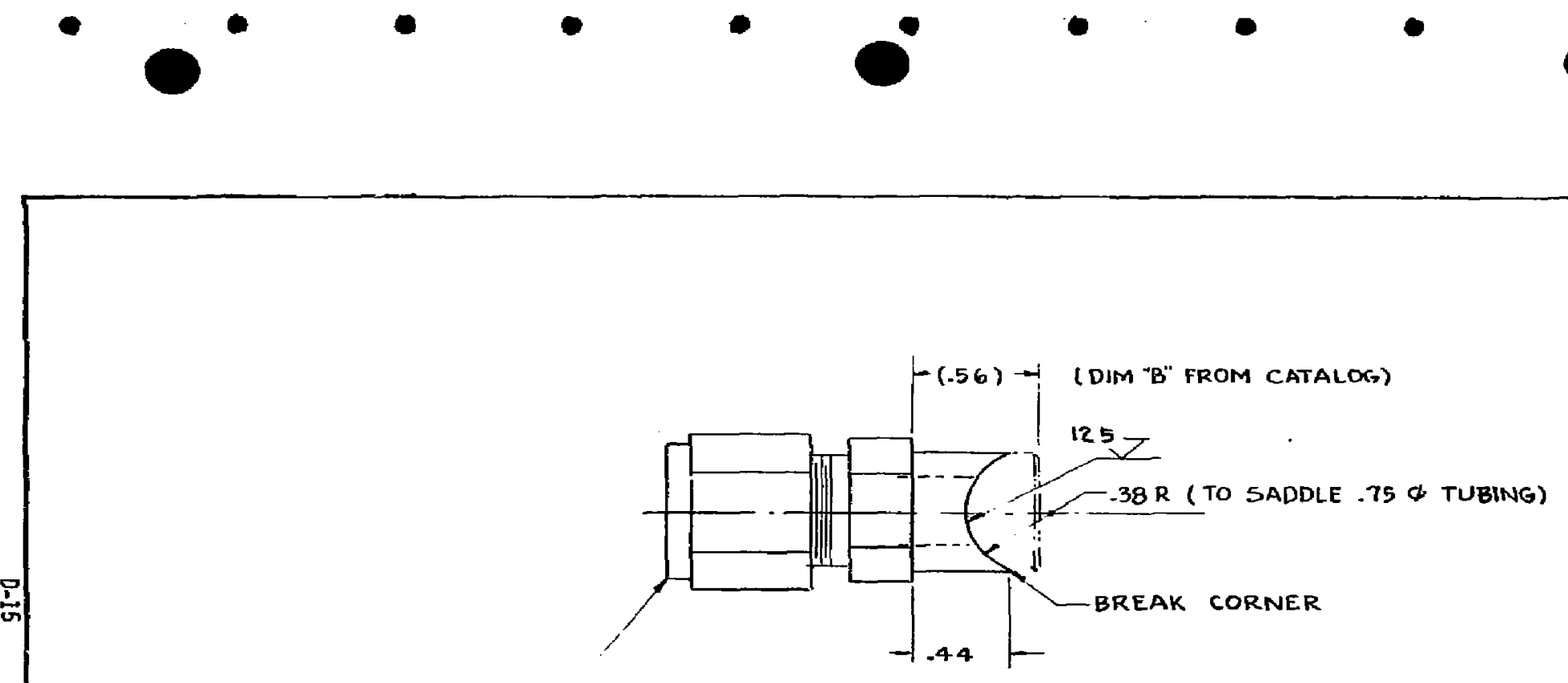

— CATALOG ITEM-- SWAGELOK $\$ 55-810-1-6 M P W$.

\begin{tabular}{|c|c|c|c|c|c|c|}
\hline & & \multirow{2}{*}{ 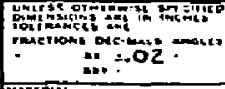 } & Fantastere & & \multicolumn{2}{|c|}{ BROBEGK ERKELEY US GALIFORNIA } \\
\hline & & & 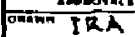 & $\frac{3016}{3 / 15 k+84}$ & \multirow{3}{*}{\multicolumn{2}{|c|}{$\begin{array}{l}\text { BEAM DIRECTOR } \\
\text { ACHROMAT } \\
\text { ANIFOLD FITTING } / 2 \text { TO } \$ / 4\end{array}$}} \\
\hline & -2 & mint & Ematy & ortes & & \\
\hline & 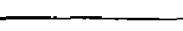 & $-\infty 31$ & & & & \\
\hline 9561743 & 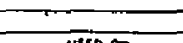 & $P \min 2 \pi$ & & & B & $95 \mathrm{~B} 1741$ \\
\hline & & DO Rar schil DAAWiNa & & & $\operatorname{senic} z=\frac{1}{2}$ & 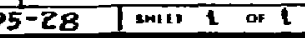 \\
\hline
\end{tabular}




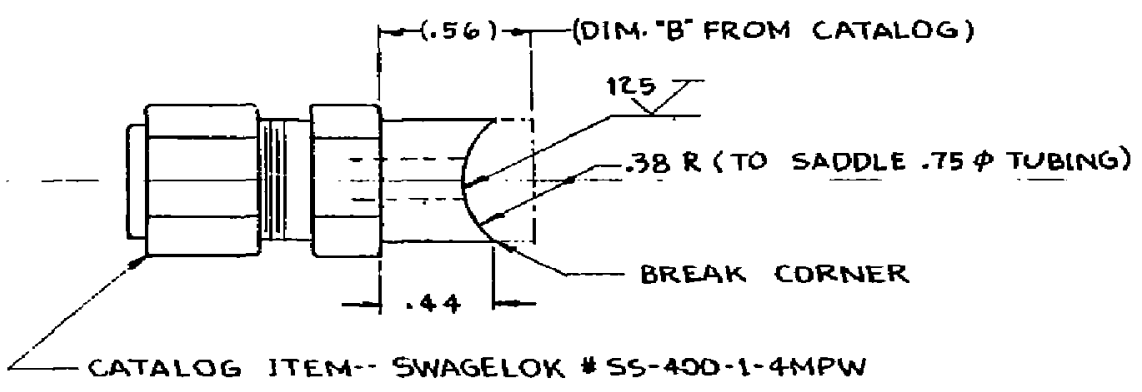

\begin{tabular}{|c|c|c|c|c|c|c|}
\hline- & & \multirow{2}{*}{ 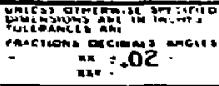 } & \multicolumn{2}{|l|}{ 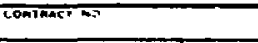 } & BAOAECK & 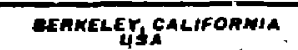 \\
\hline & & & IRA & $3 / 15 / 84$ & BEAM & DIRECTOR \\
\hline & & & 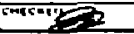 & ores & & \\
\hline & 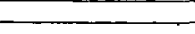 & S5T & & & IIFOLD & $1 / 4$ to $3 / 4$ \\
\hline 9501743 & 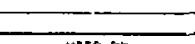 & & & & $B$ & $95 B 1742$ \\
\hline
\end{tabular}




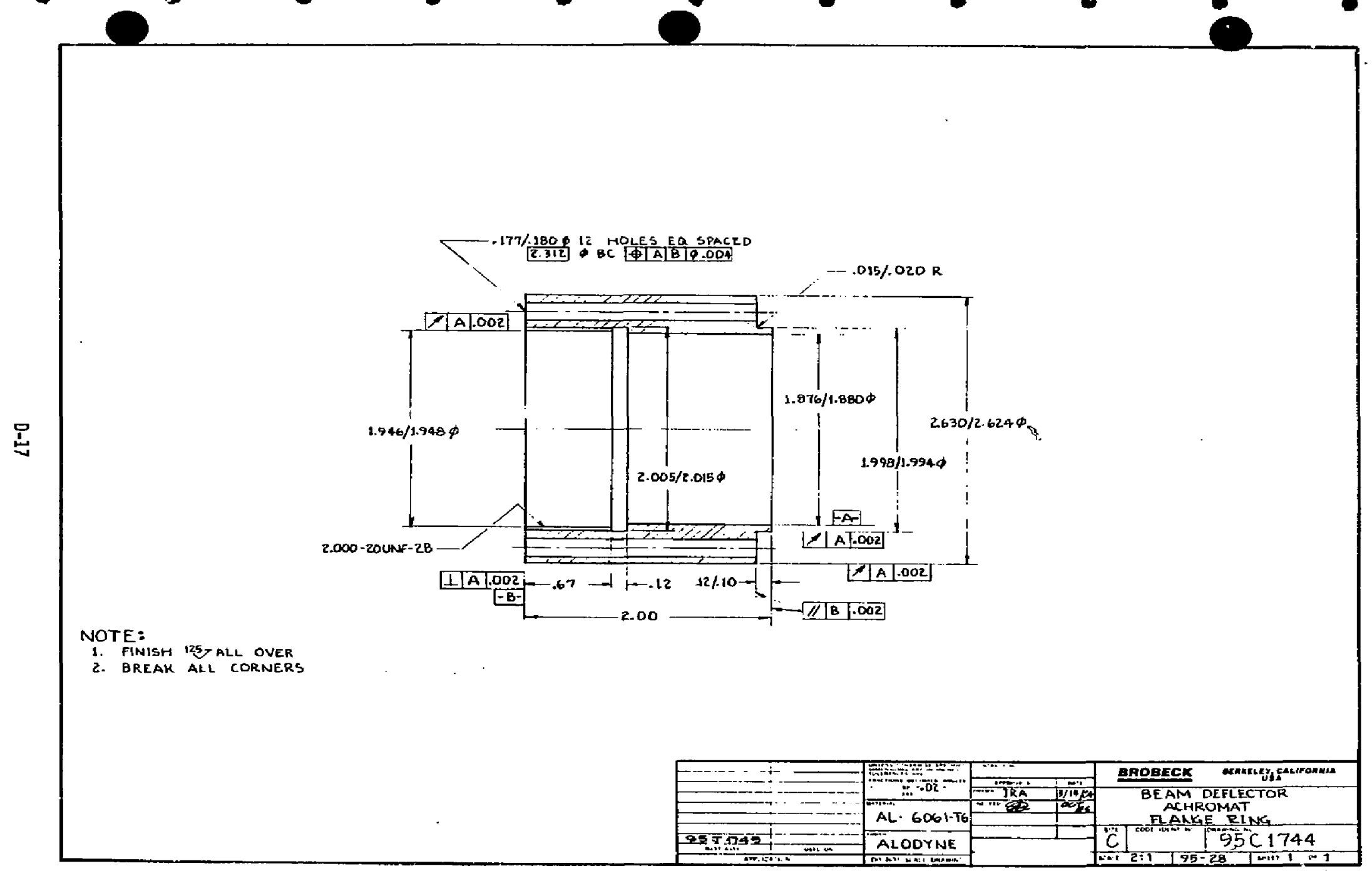


$.177 / .180 \Phi 12$ HOLES EQ SPACED 2.312 BC \begin{tabular}{|l|l|l|l|}
\hline$\perp$ & $A$ & $B$ & $\$ .004$ \\
\hline
\end{tabular}

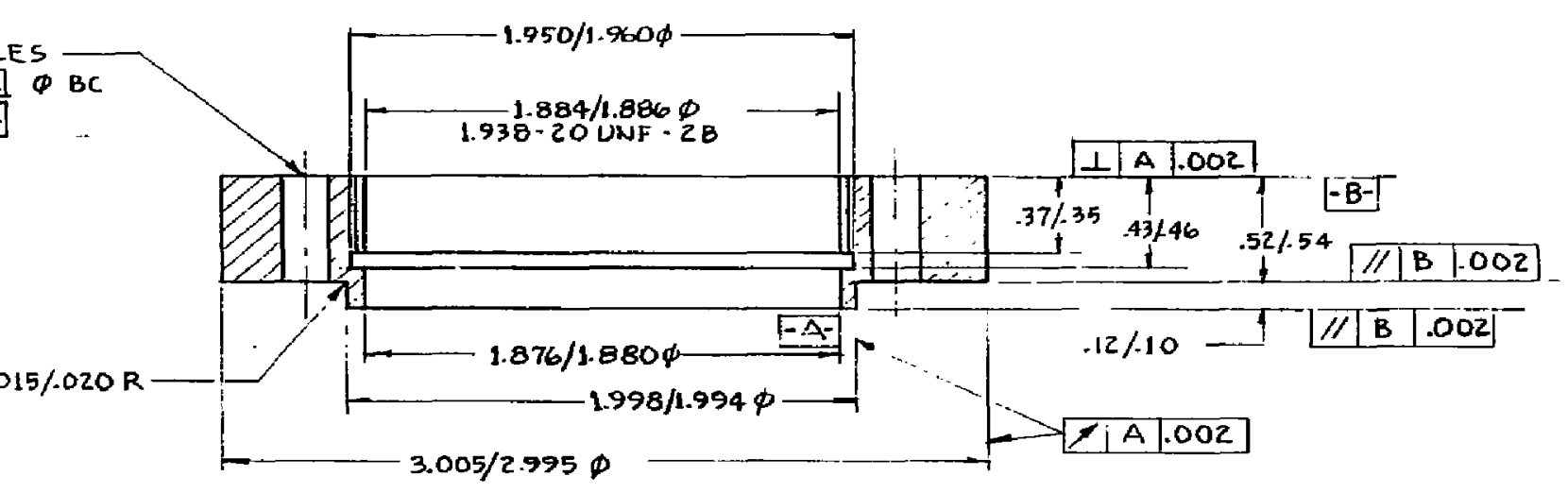

NOTE:

1. FINISH 125 ALL OVER.

2. BREAK ALL CORNERS.

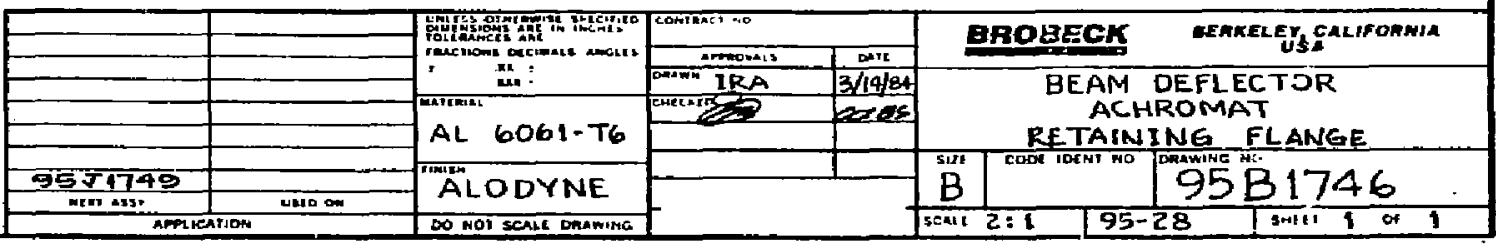




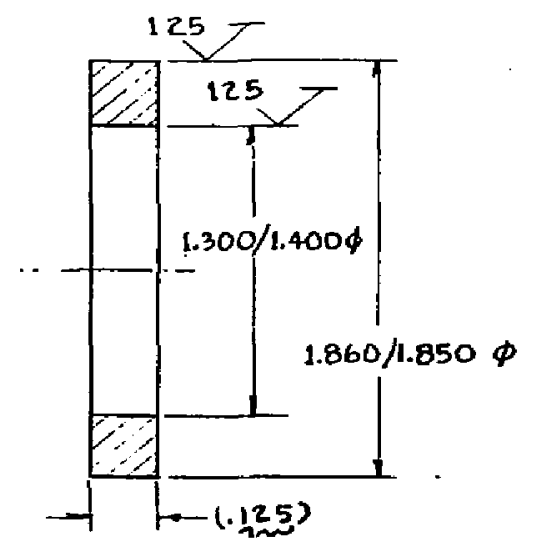

\begin{tabular}{|c|c|c|c|c|c|c|}
\hline & & 4 & \multicolumn{2}{|l|}{ 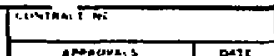 } & \multicolumn{2}{|c|}{ DAOEECKE BERKELEY IS CALIFORN:A } \\
\hline & & 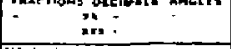 & minn IPA & $3 / 144$ & \multirow{3}{*}{\multicolumn{2}{|c|}{$\begin{array}{l}\text { BEAM DIRECTOR } \\
\text { ACHROMAT } \\
\text { BARRLER RING }\end{array}$}} \\
\hline & & $G-1,125 \mathrm{THK}$ & manges & ass & & \\
\hline & & & & & & \\
\hline DS+1749 & - & & & & B & $95 B 1748$ \\
\hline 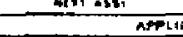 & 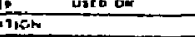 & 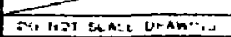 & & & $\therefore-2: 1$ & $28 \mid \operatorname{sen} 1+1$ \\
\hline
\end{tabular}




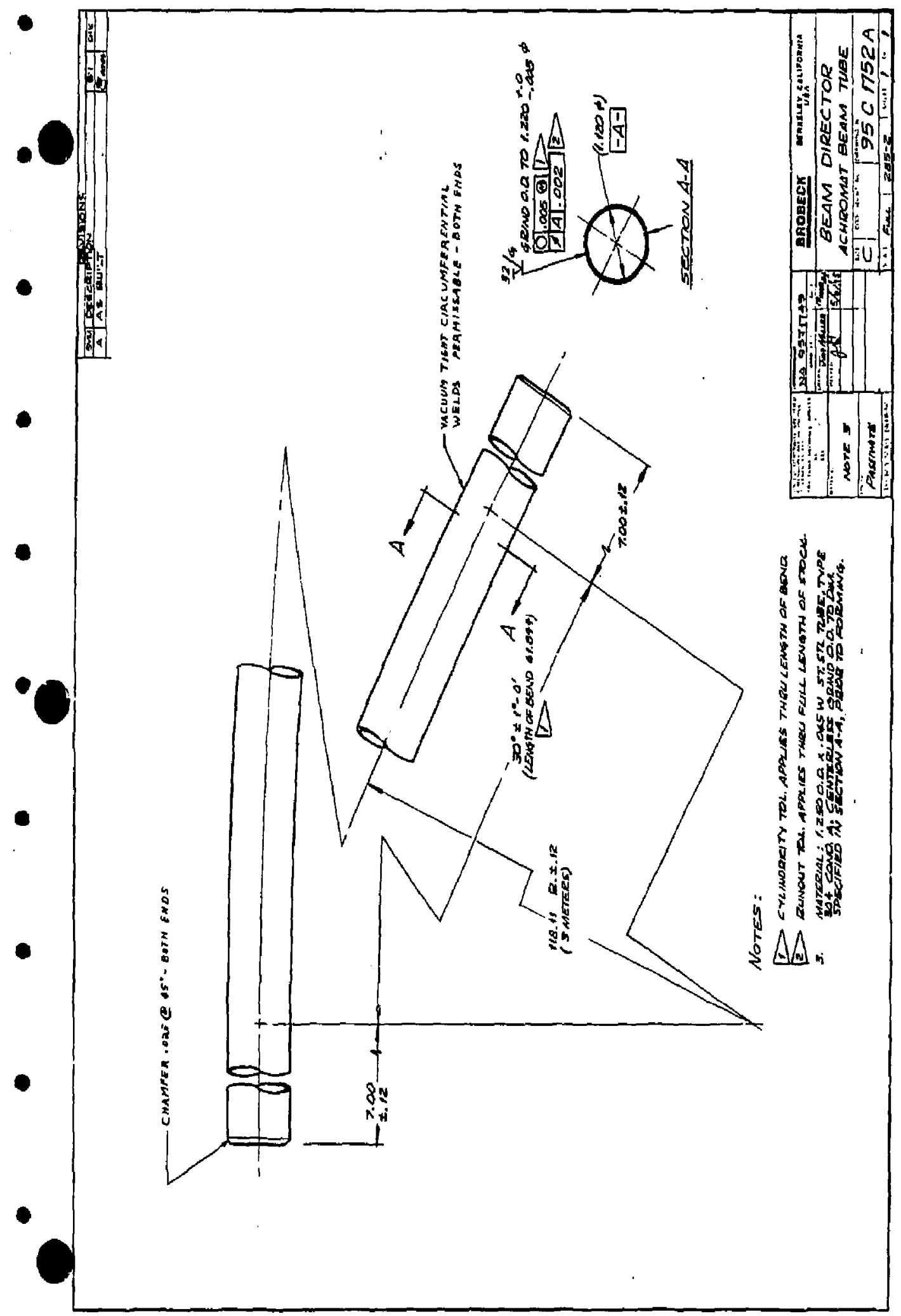




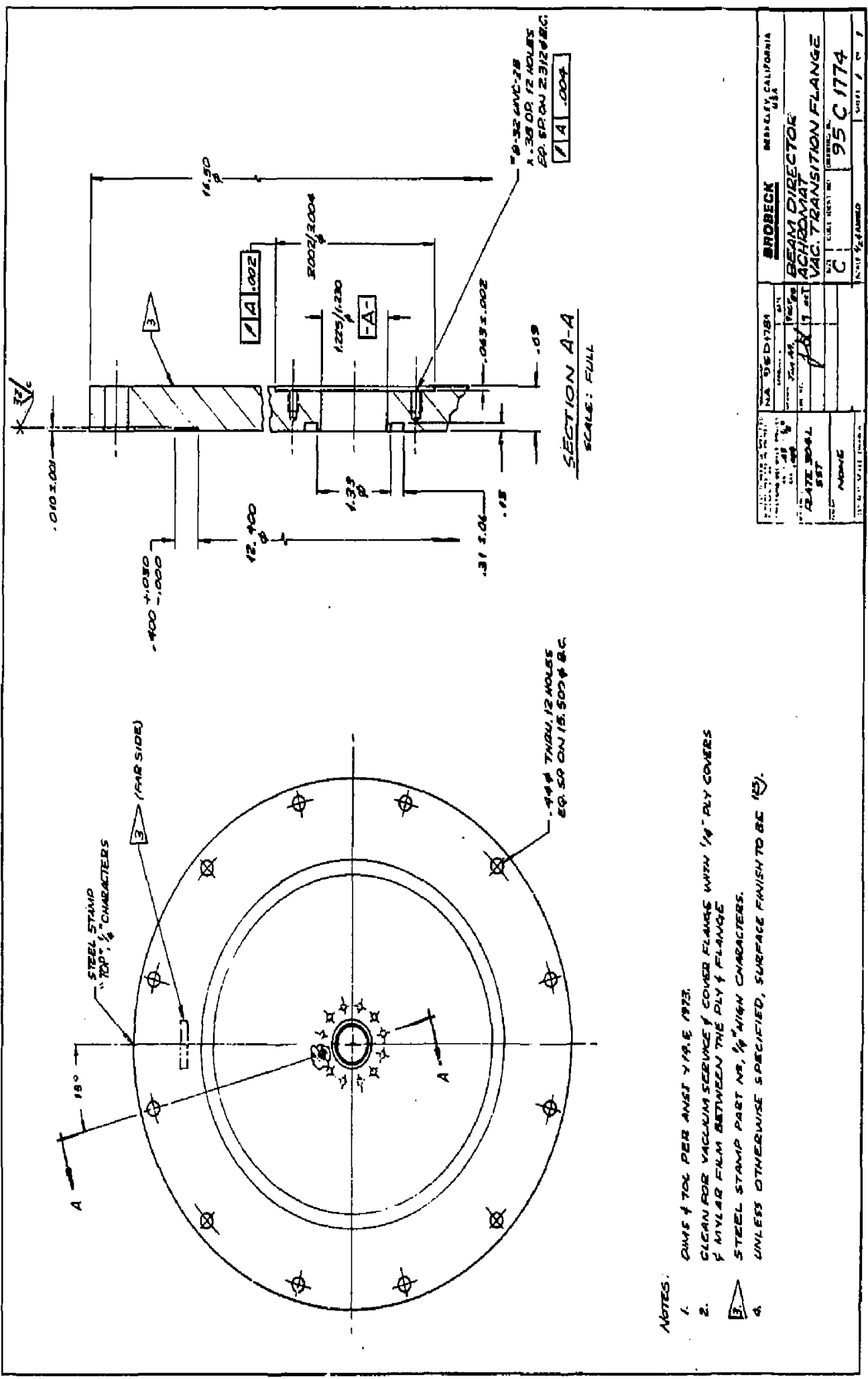




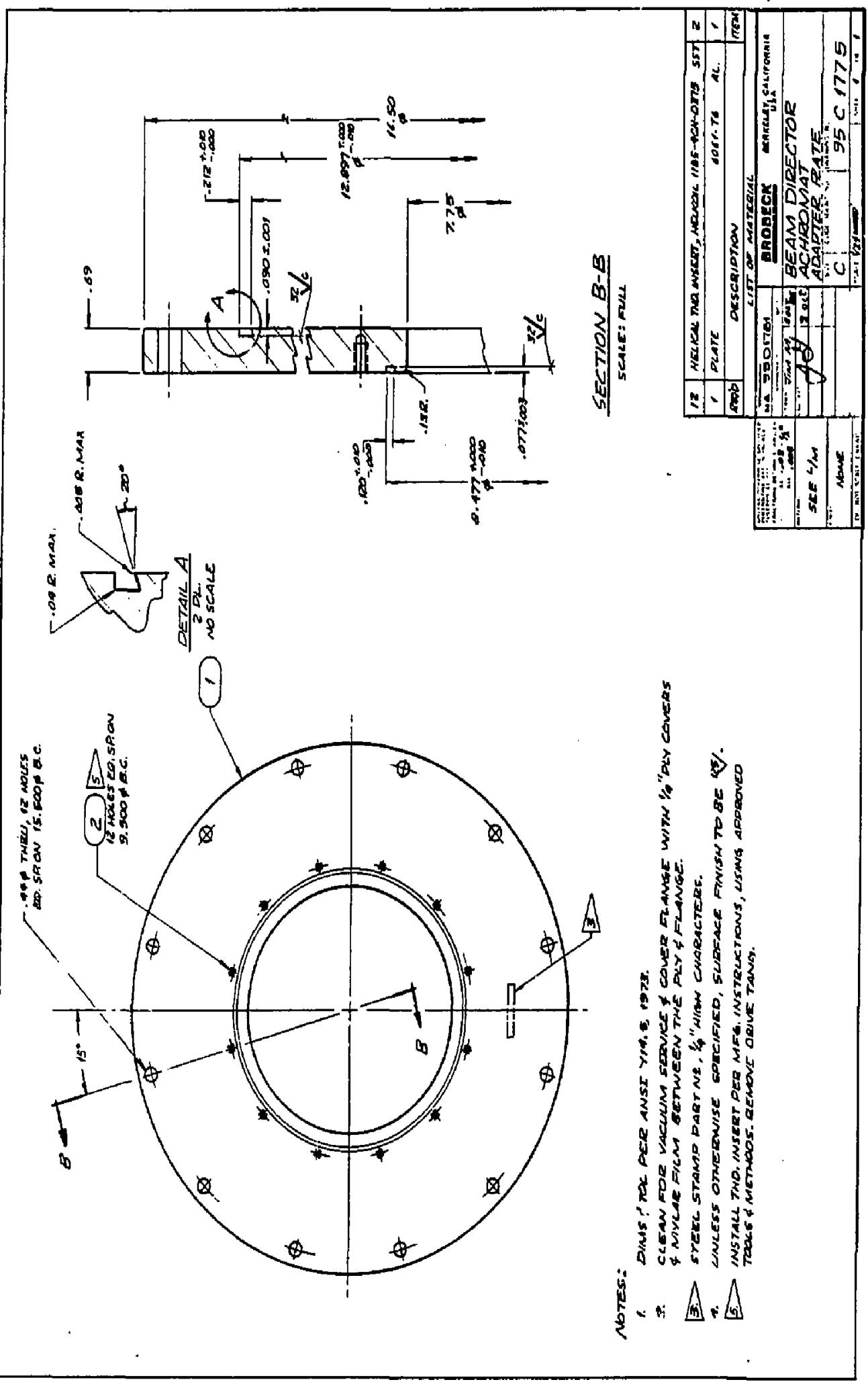



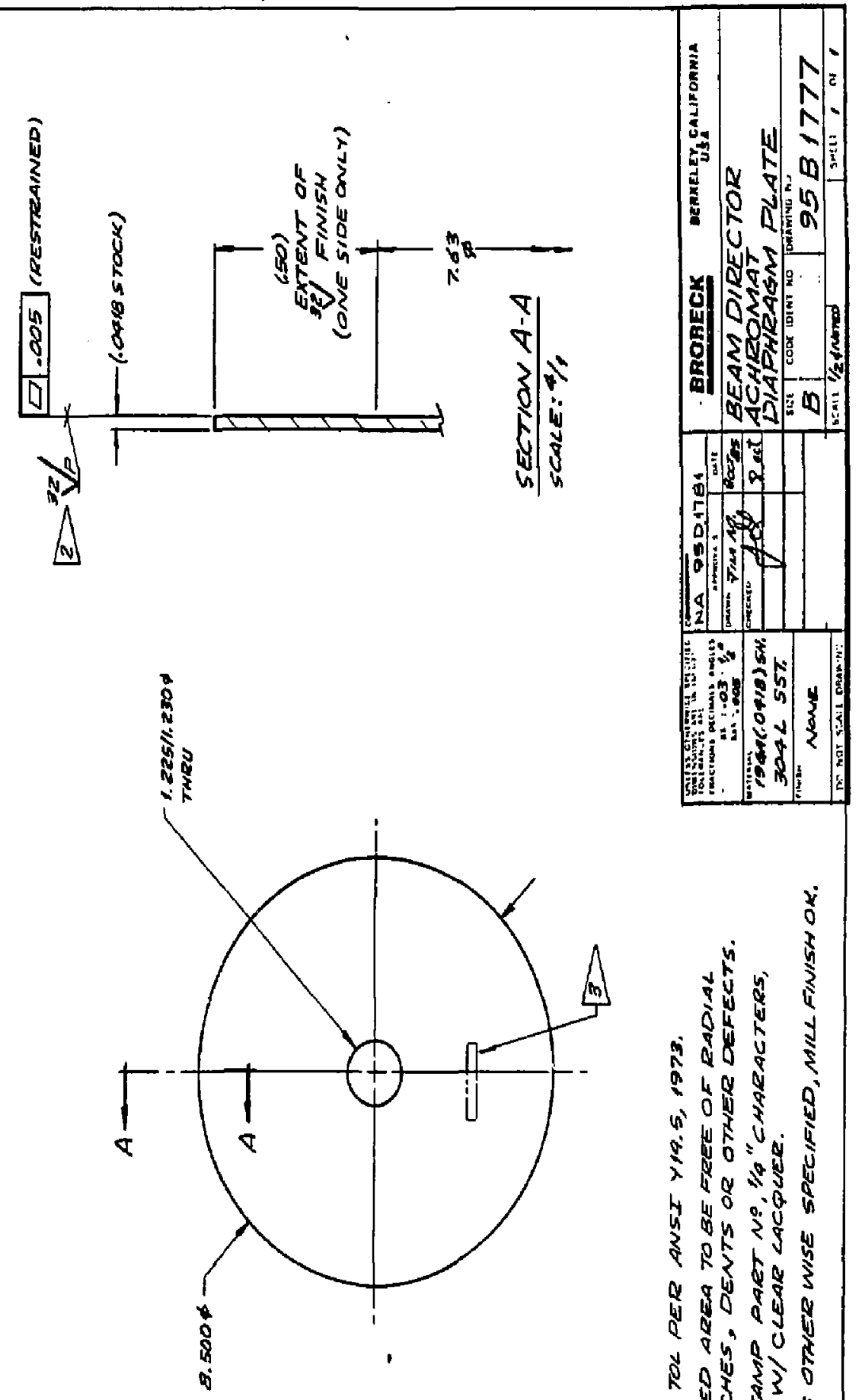


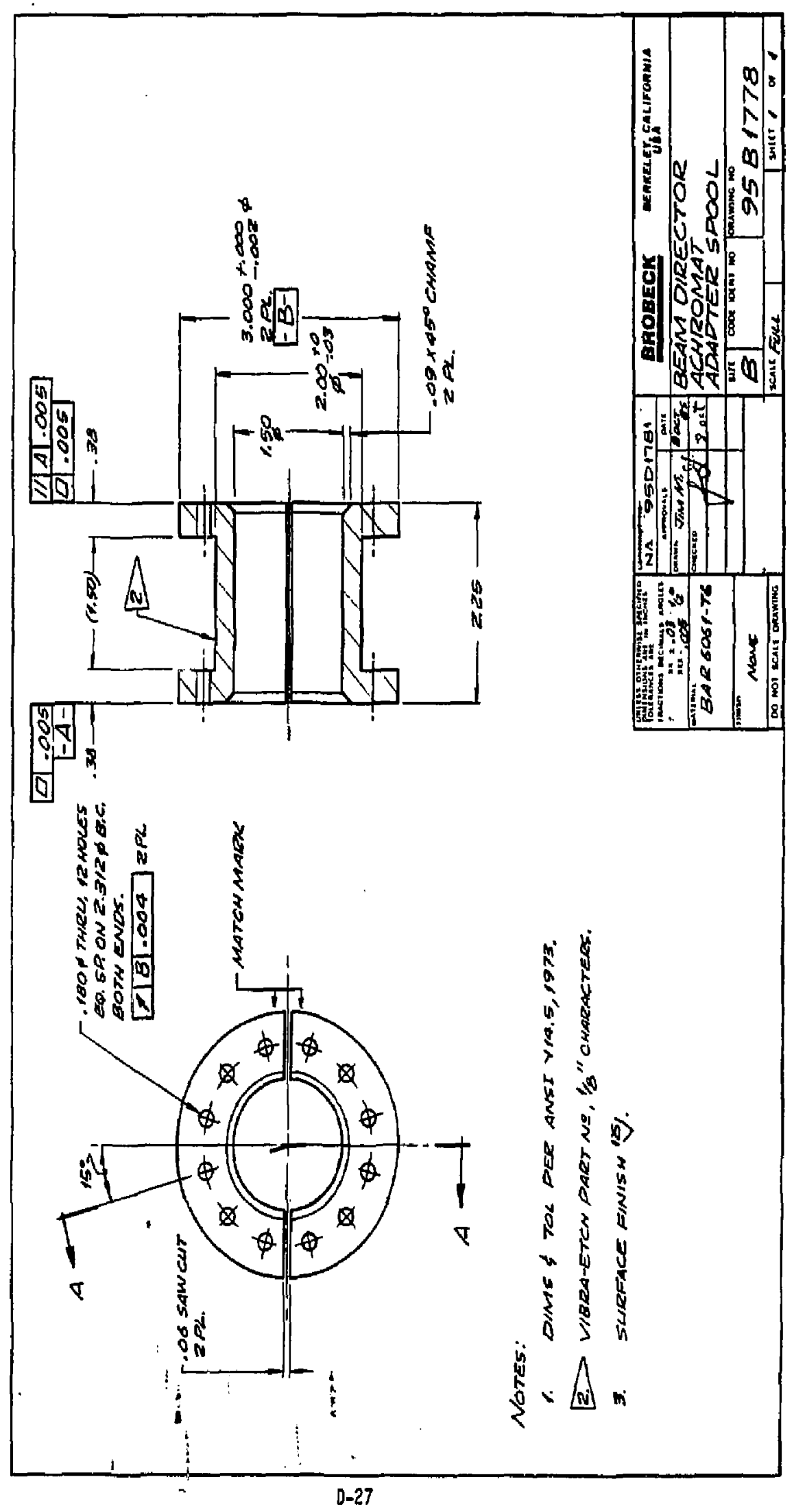




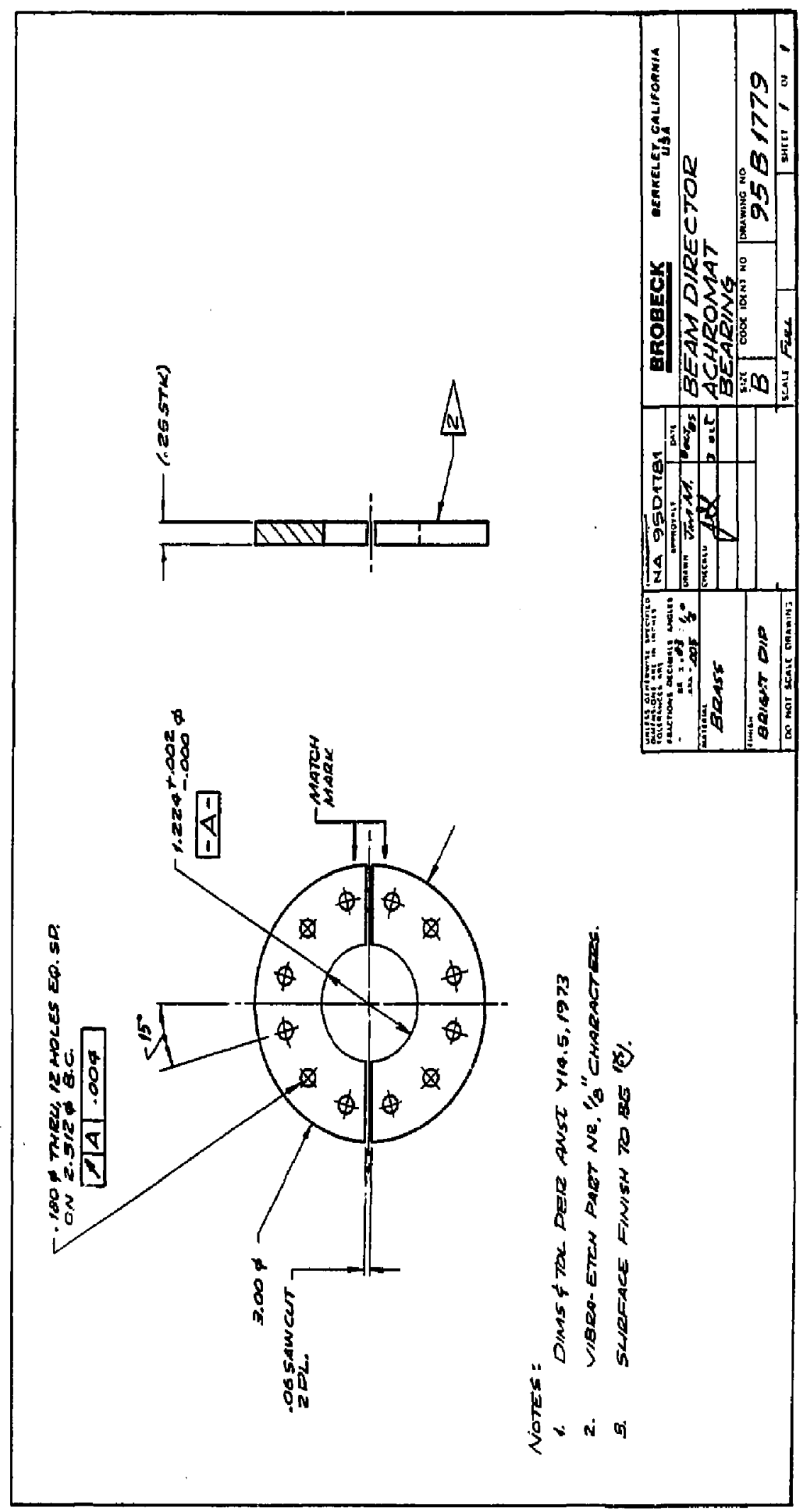




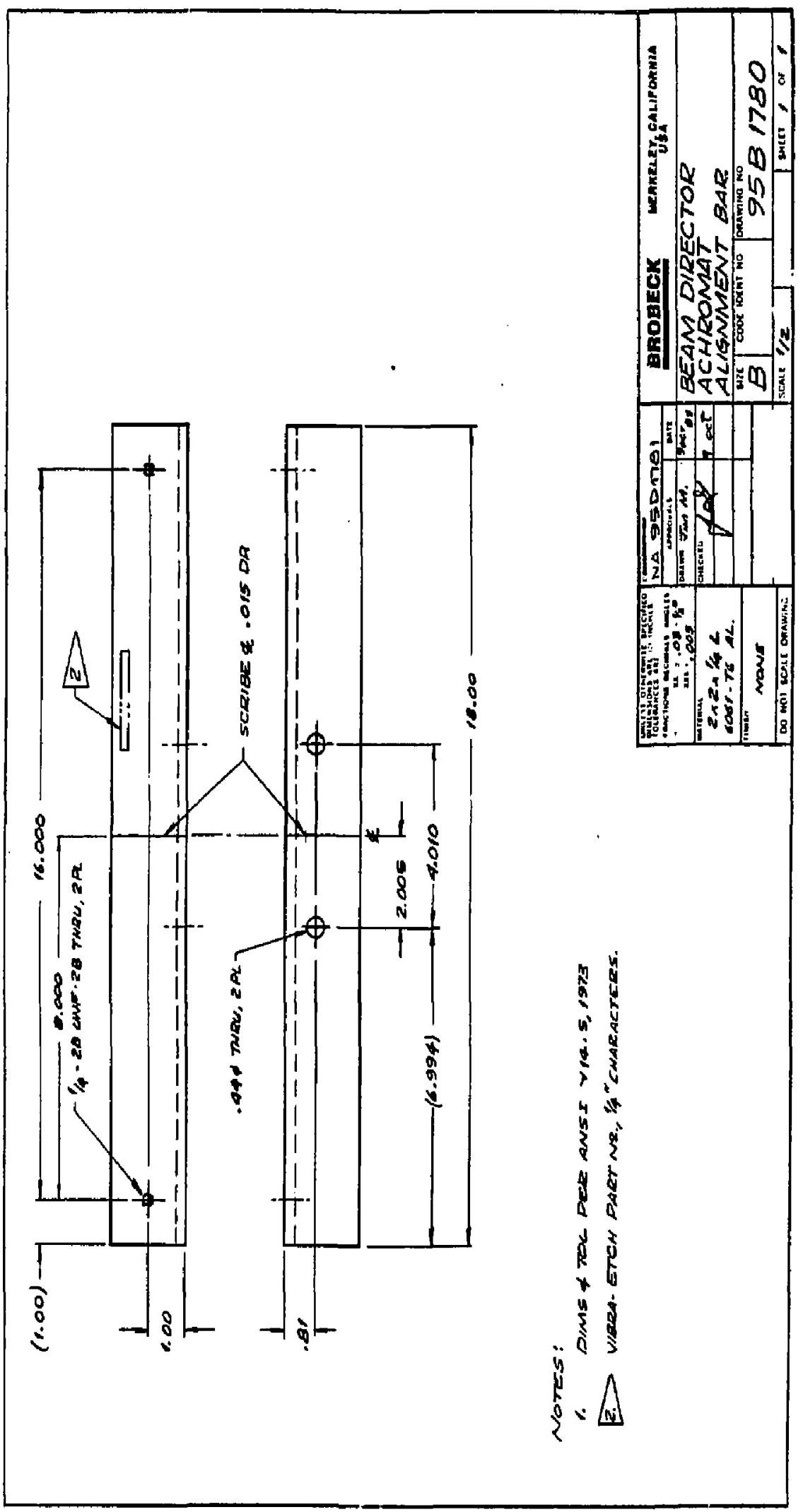




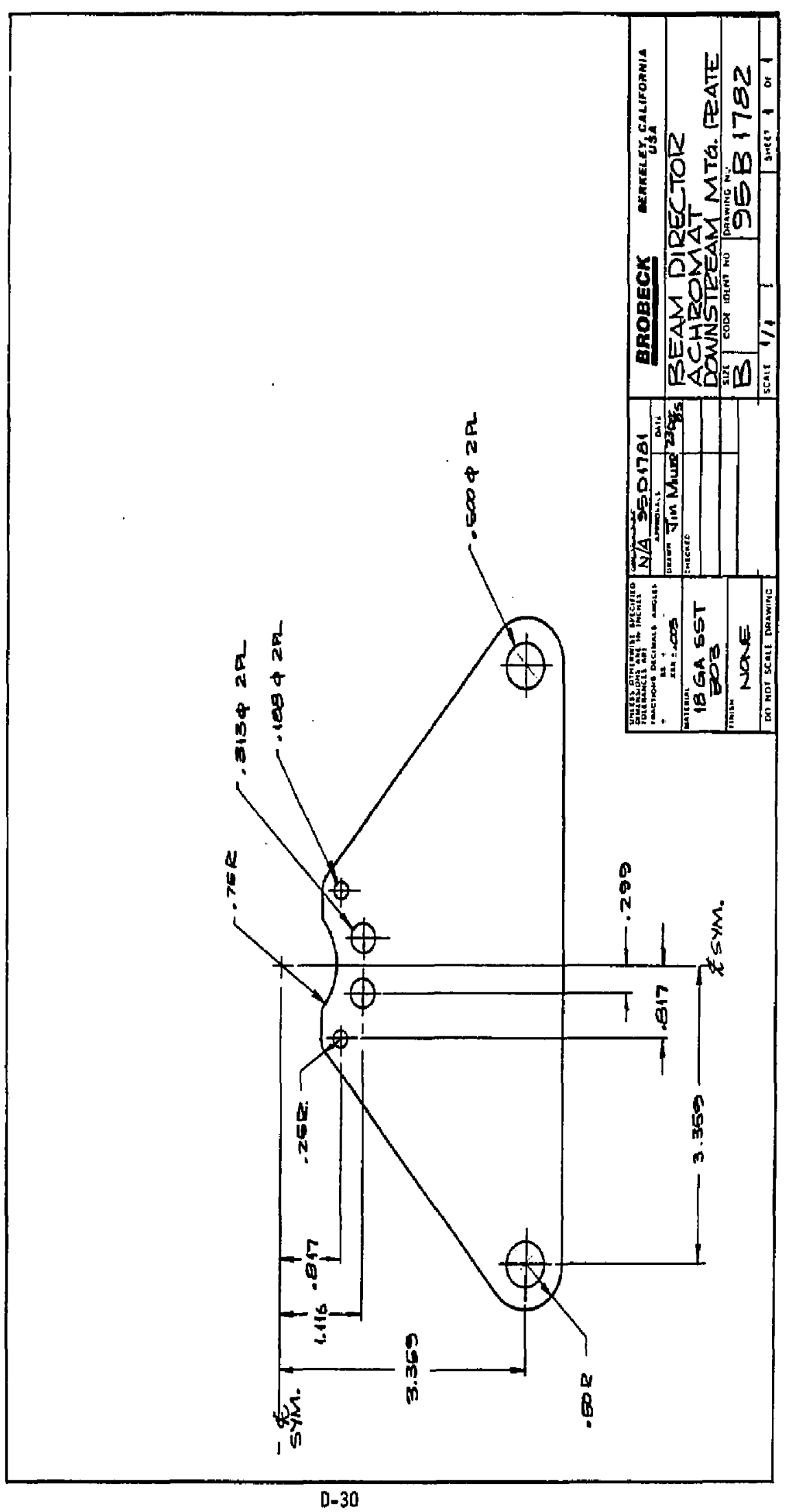




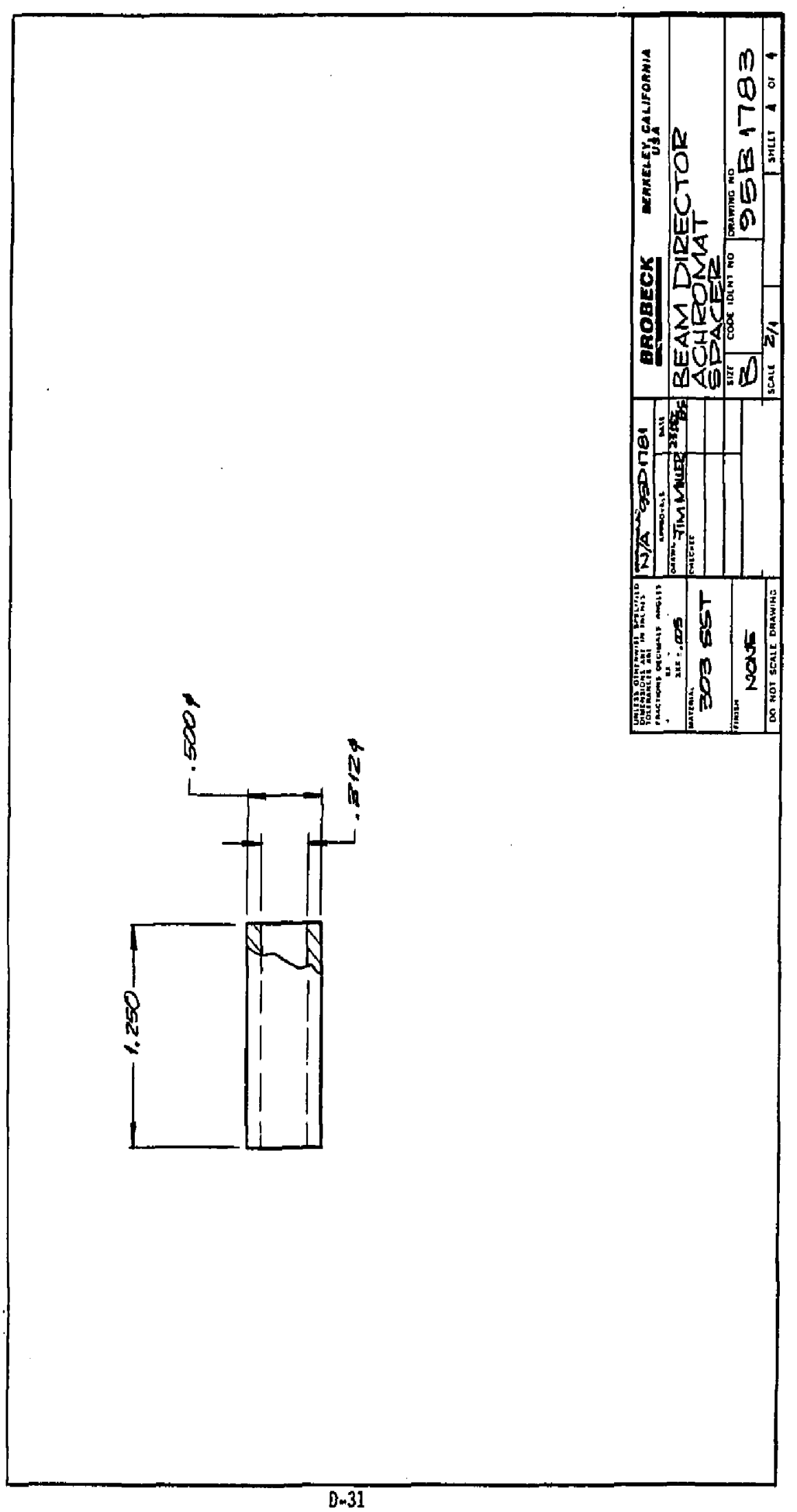




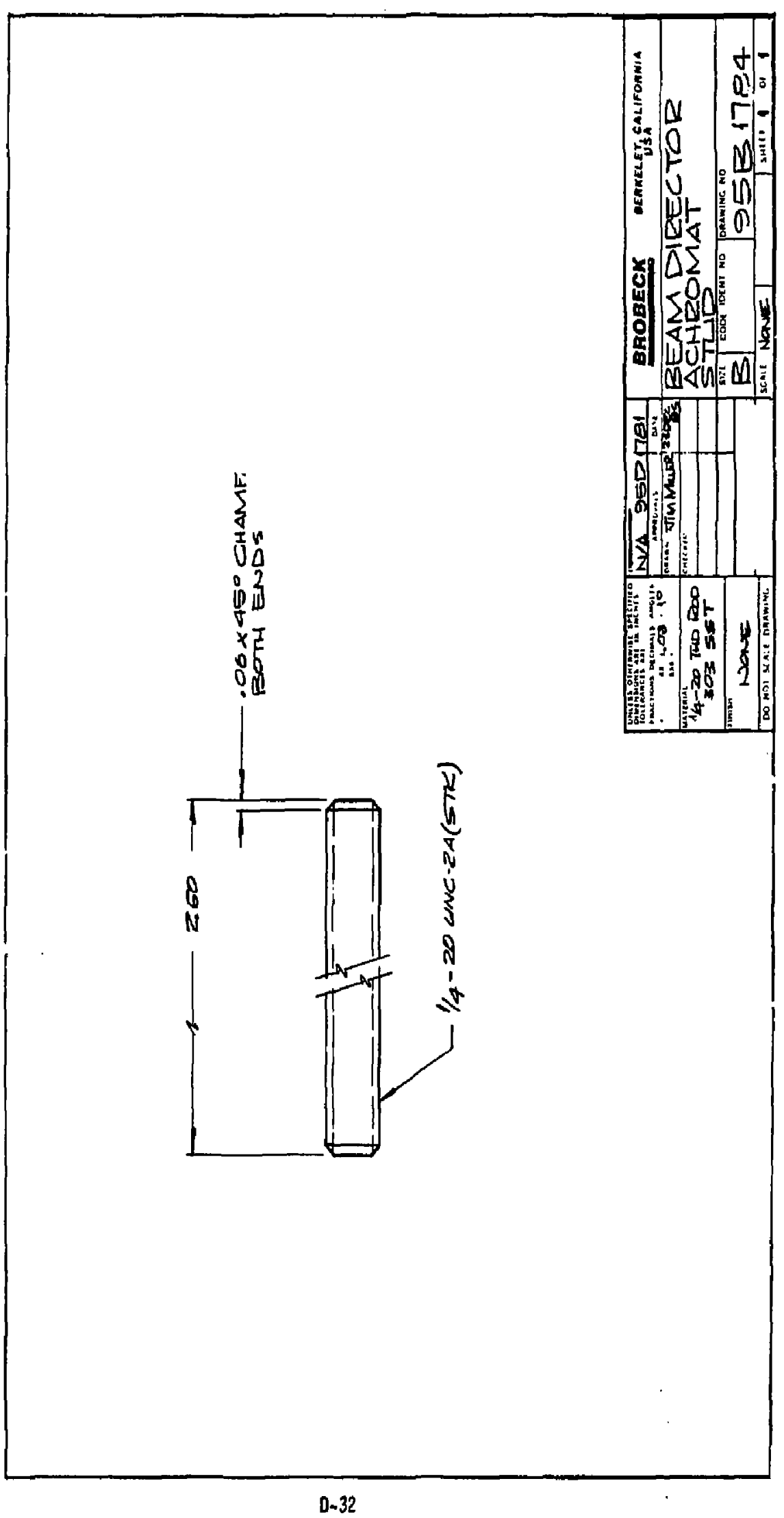




\section{APPENDIX E. DRAWINGS: VERNIER}

A complete set of as-built drawings for the vernier is included in this appendix.

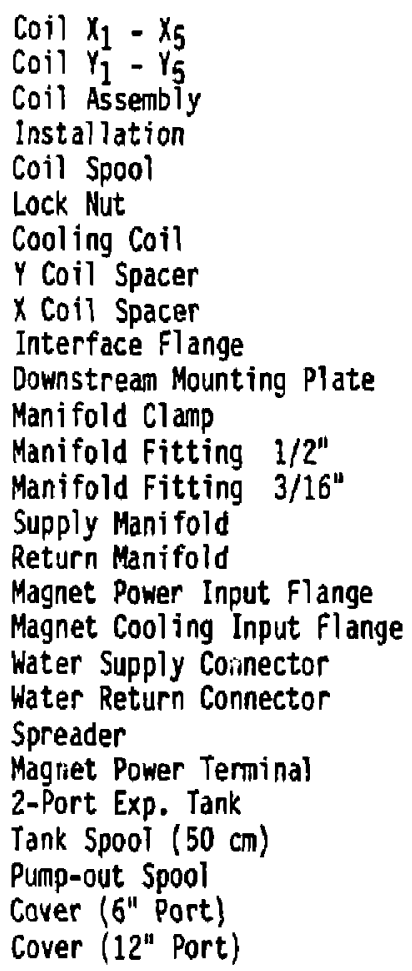

9501705

9501706

95E 1710

9501728

9501707

95C1708

95C1709

95C1712

95C 1713

$95 E 1714$

95E1716

$95 B 1717$

$95 \mathrm{~B} 1718$

$95 \mathrm{~B} 1719$

9501720

9501721

95C1723

$95 \mathrm{C} 1724$

9581725

9581726

9581727

$95 B 1729$

9501769

9501770

9501771

9501772

95C1773 

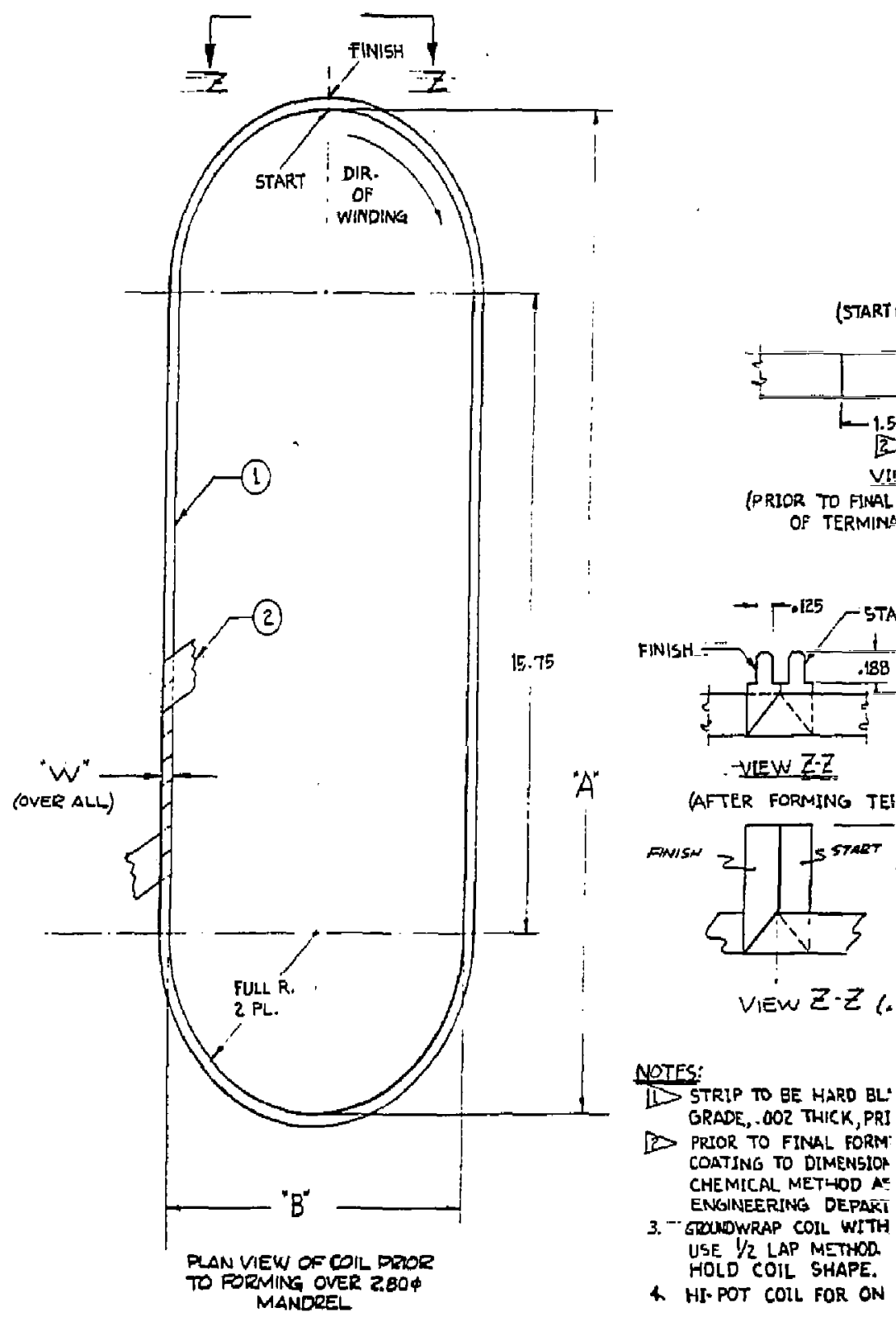

(PRIOR TO FINAL OF TERMIN

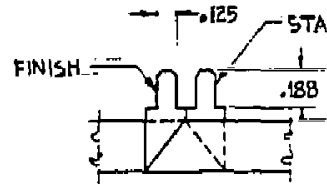

DIEW ZZZ

LAFTER FORMING TEI FNOSN

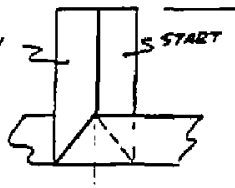

VIEW $z \cdot z$.

\section{NOTES:}

ID STRIP TD BE HARD BL: GRADE, , 002 THICK, PRI $D$ PRTOR TO FINAL FORM COATING TO DIMENSIOP CHEMICAL METHOD AE ENGINEERIALG DEPAKTI

3. - GDONDWRAP COIL WITH USE $1 / 2$ LAP METHOO. HDID COIL SHAPE.

4. HI- POT COIL FOR ON

5 NOMINAL COILDESIGN I FOR $Z$ " BEND O 4SMEY 


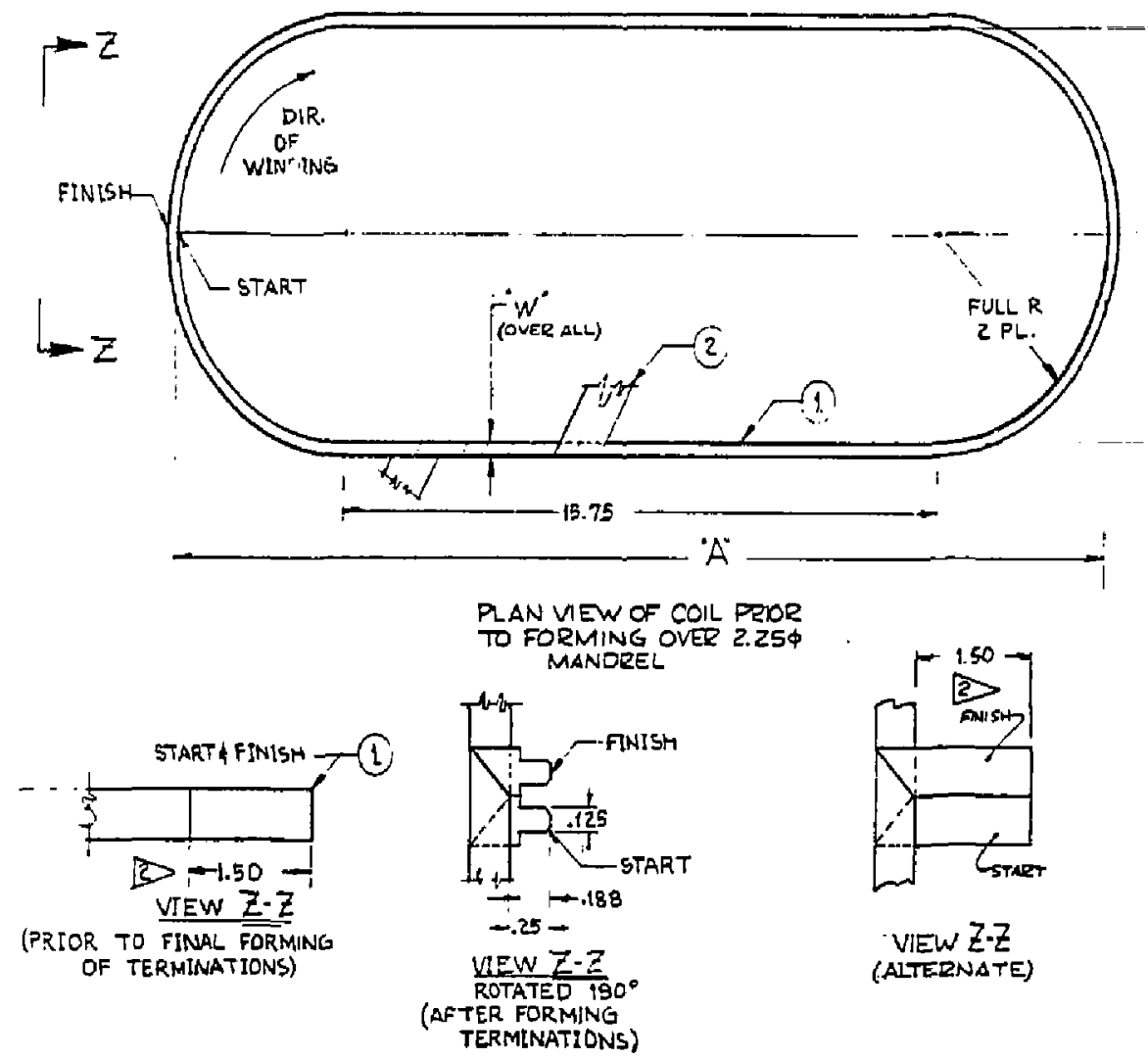

NOTES:

ID STRIP TO BE MARD BLACK ANODIZED, ELECTRICAL INSULATION GRADE, .0O2 THIRK, PRLOR TO WIMDING COLL.

D PRIOR TO FINAL FORMING OF TERMINATIONS, STRIP ANOORED COATING TO DIMENSION SPECIFTED. USE MECHANICAL OR CHEMICAL METHOD AS APPROVED BY GROBESK CORPORAT ION ENGINEERING DEPARTMENT.

3 GRUOWRAP COIL WITH MMAR TAPE, ITEM 2 , AFTER FORMING. USE $/ 2$ LAP METHOD. USE MINMAL TAPE TENSION TO HOLD COLL SHAPE

4. HI-POT COLL FO, ONE (1) MINUTE.

5 NOMINUL COLL OESIGN IS 20.830 AMPS TO GNE 132 GAUSS DOZ 2 BENDC $45 \mathrm{MeV}$. 


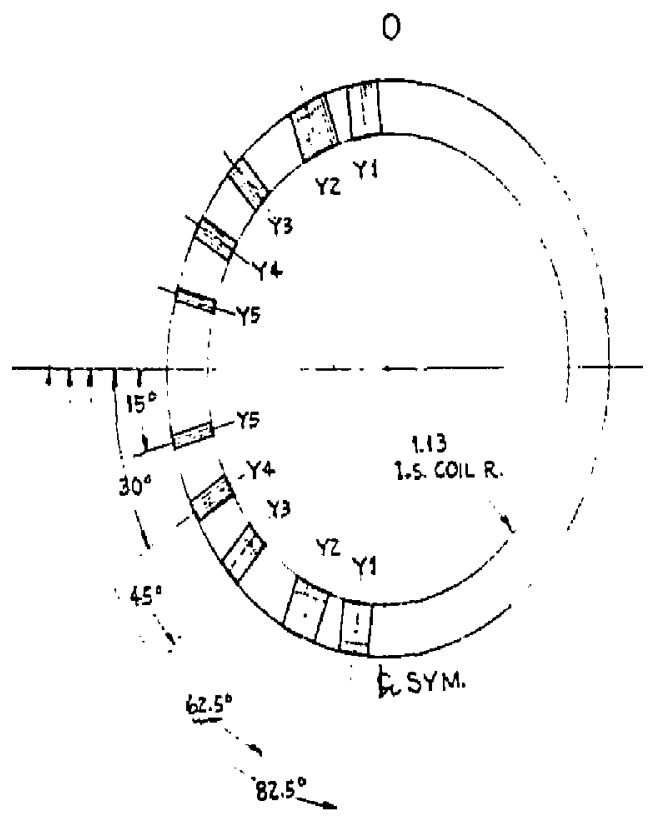

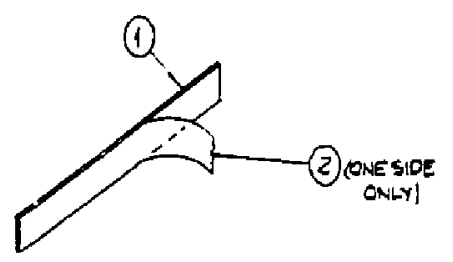

COLL STRIP PREP.

(DRIOR TO WINDING)

\begin{tabular}{|c|c|c|c|c|c|c|}
\hline \multicolumn{7}{|c|}{ TABULATION } \\
\hline COIL & DIM A & lotM B & $\begin{array}{l}\text { NO. OF } \\
\text { TURNS }\end{array}$ & PART NO. & $\begin{array}{l}\text { RER'D } \\
\text { PER ASSY }\end{array}$ & DIM W \\
\hline$\overline{Y I}$ & $10130^{\circ}$ & 3.390 & 11 & $9501706-1$ & $i$ & .272 \\
\hline$\sqrt{2}$ & 19.47 & 2.467 & 13 & $950179-2$ & 2 & .372 \\
\hline$y^{3}$ & $17.5 \%$ & $1.52^{2}$ & 7 & $9501706-3$ & 2 & .176 \\
\hline Y4 & 16959 & 1.209 & 5 & 95017064 & 2 & .128 \\
\hline$Y \xi$ & 162344 & .594 & 3 & $9501700=E$ & 2 & .080 \\
\hline
\end{tabular}

PAGE

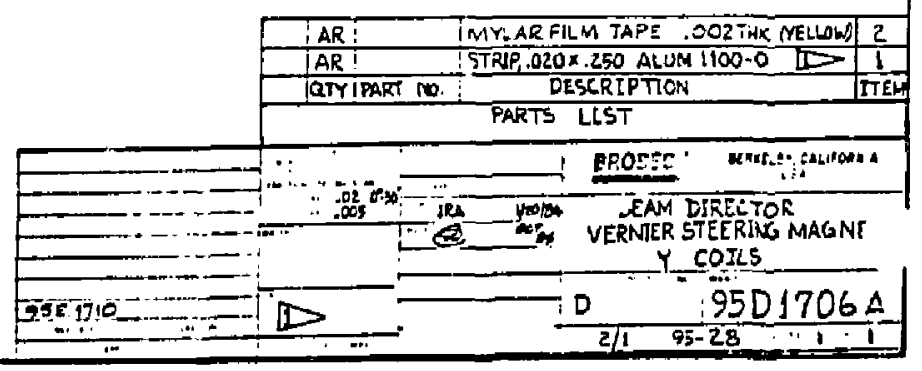



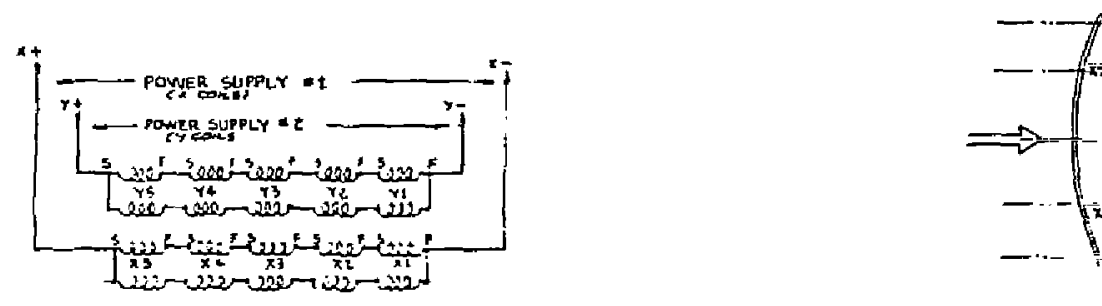

co:

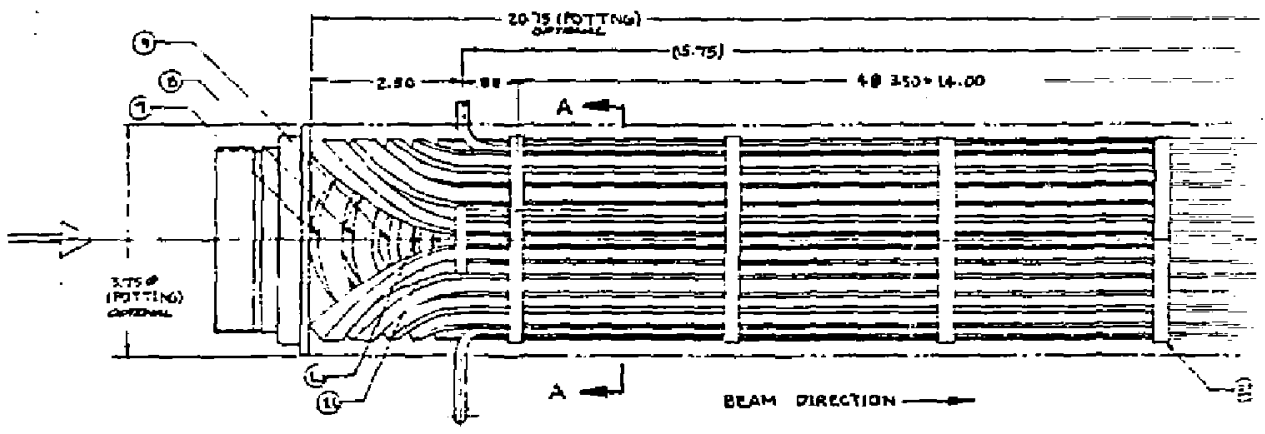

№tr:

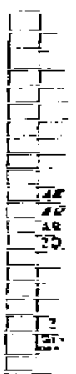




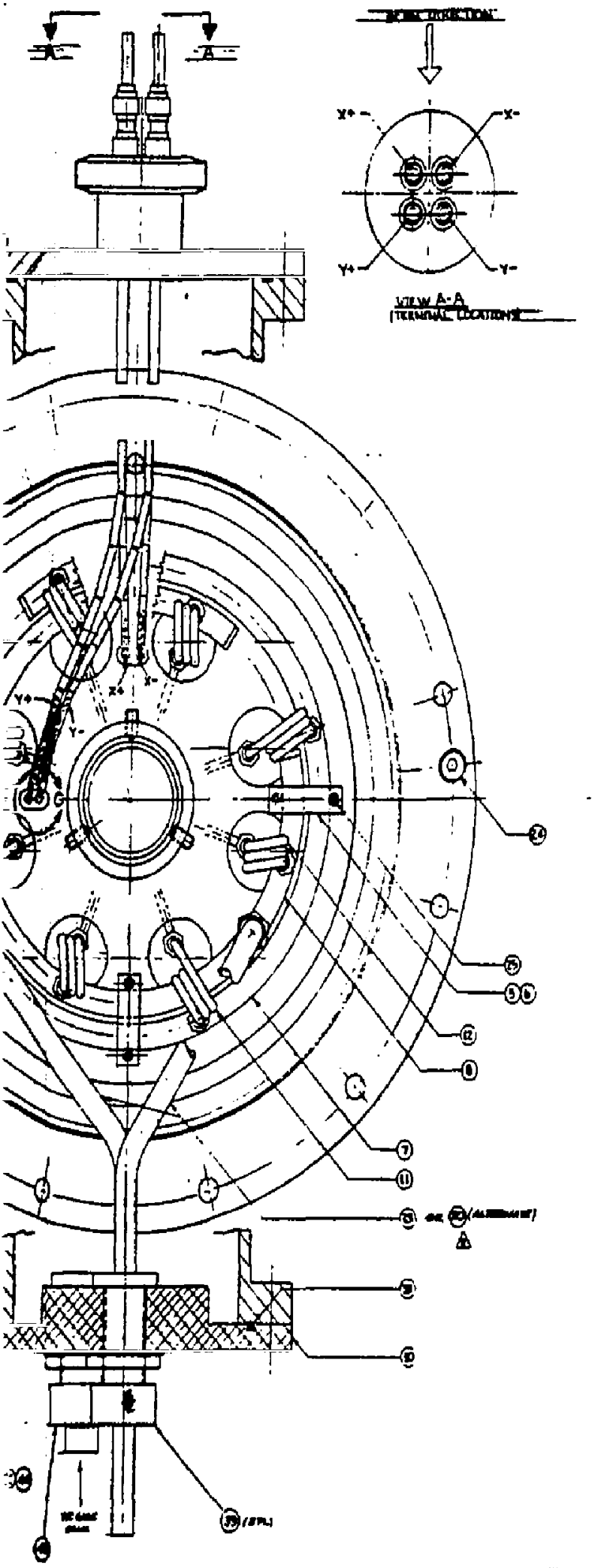

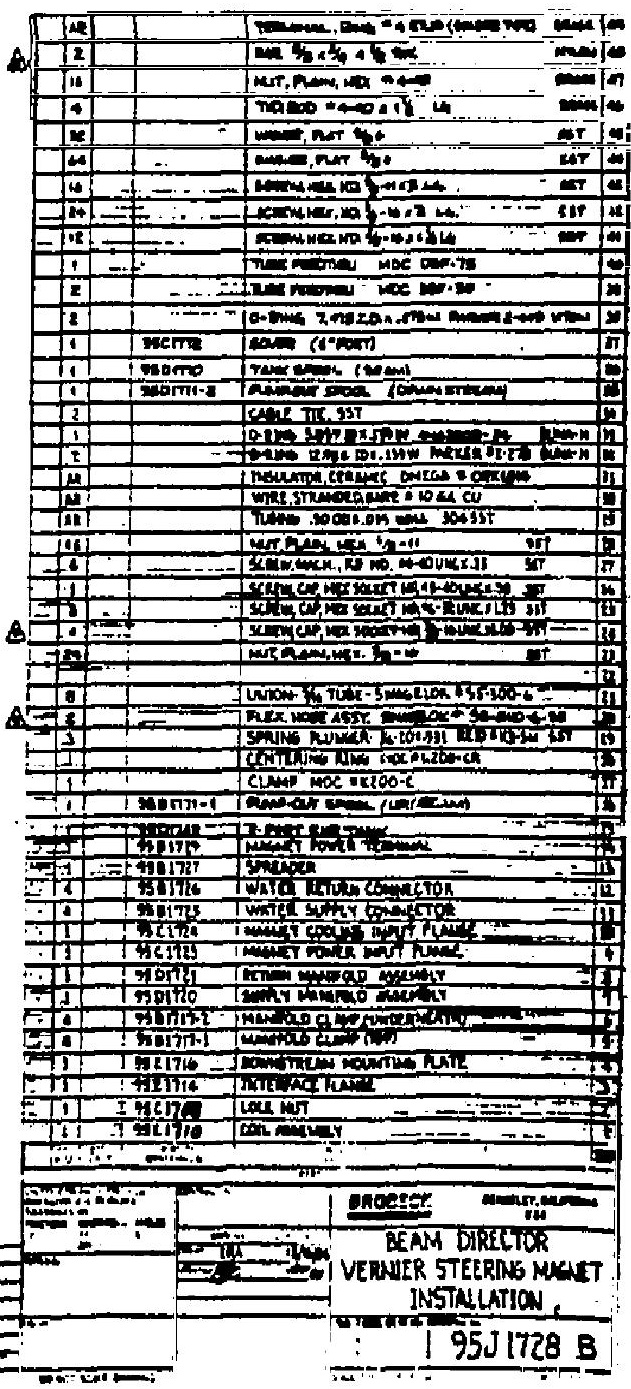




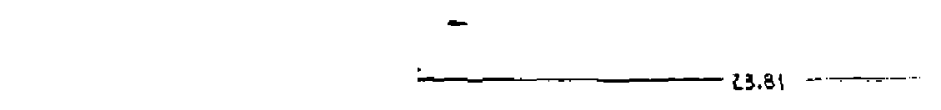

1

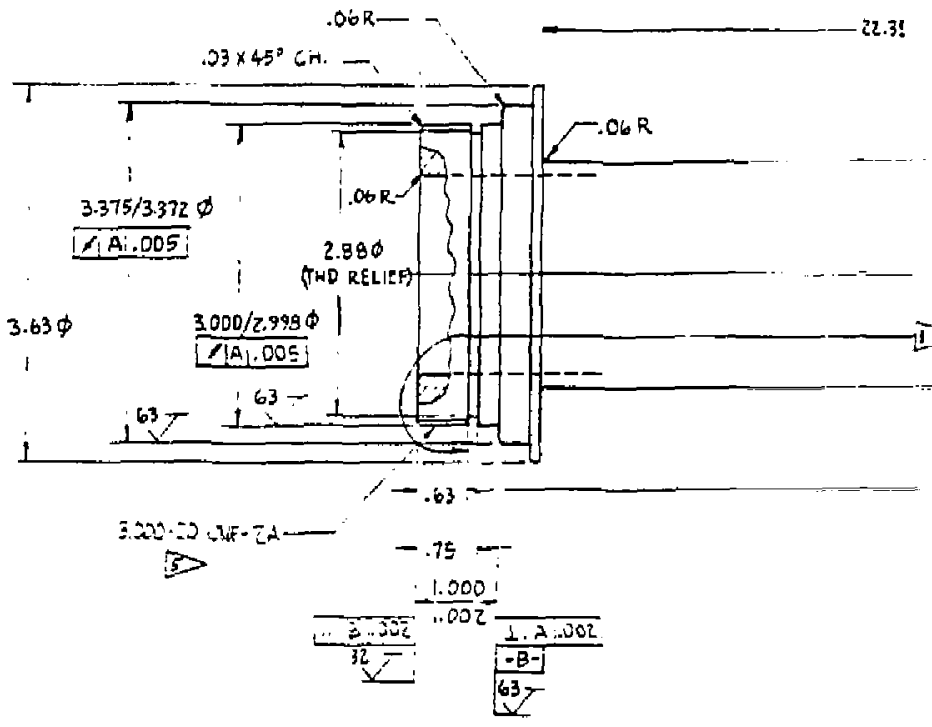

NOTES:

DS SILVER PLATE AREA INDICATED .0OI THK. MIN.

-2. ALL CORNER .O3 R EXCEPT AS INDICATED.

3. ALL SURFACES J25- EXCEPT AS INDICATED.

I5 HARD BLK ANODIZE AREA INDICATEO, ELEETRICAL jKSULLATION GRADE, .OCZ THK.

E) THIS PART MATES WITH $95 C$ MOS

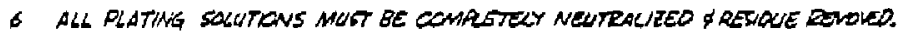



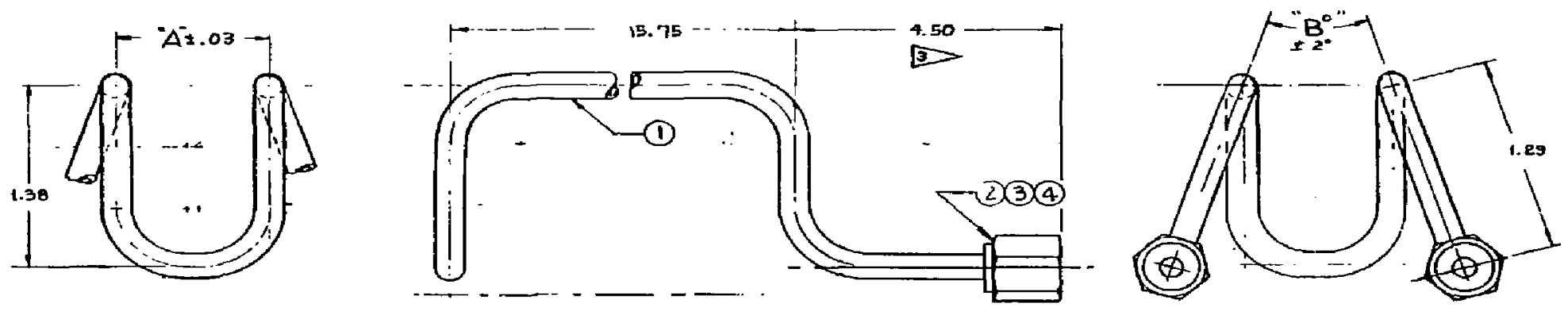

NOTE:

1. ALL BEND RADII $=.44$

i. ALL BEND RADII $=.44$.4

ITENAS 324 ARE INSTALLED PER MANUFACTURER'
INSTRUCTIONS USING A SWAGELOK PRE-SWAGING
TOOL.

BS THIS DIMENSION IS WITH NUT AGAINST SWAOED ON FERRULES.

\begin{tabular}{|c|c|c|c|}
\hline \multicolumn{4}{|c|}{ TABULATION } \\
\hline PART N9 & DINA & ANELEE & QTK \\
\hline $95 C 1709-1$ & .97 & $34^{\circ}$ & 2 \\
\hline $95 C 1709-2$ & 1.24 & $14^{\circ}$ & 2 \\
\hline
\end{tabular}

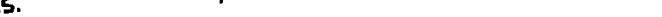

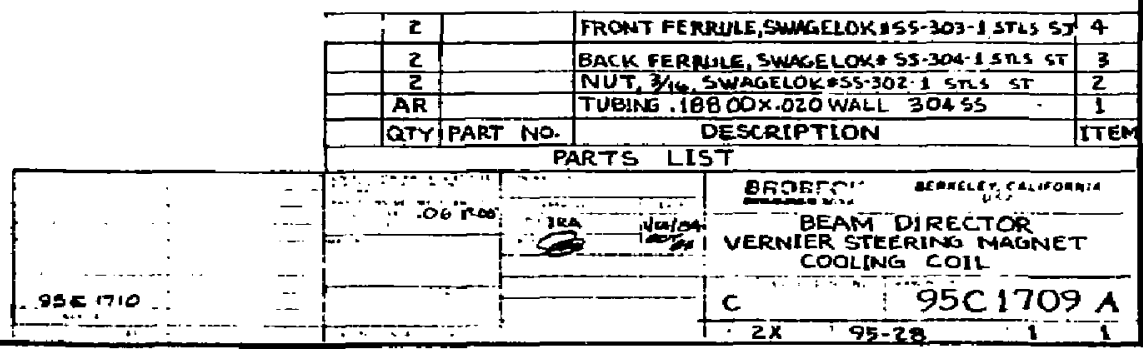




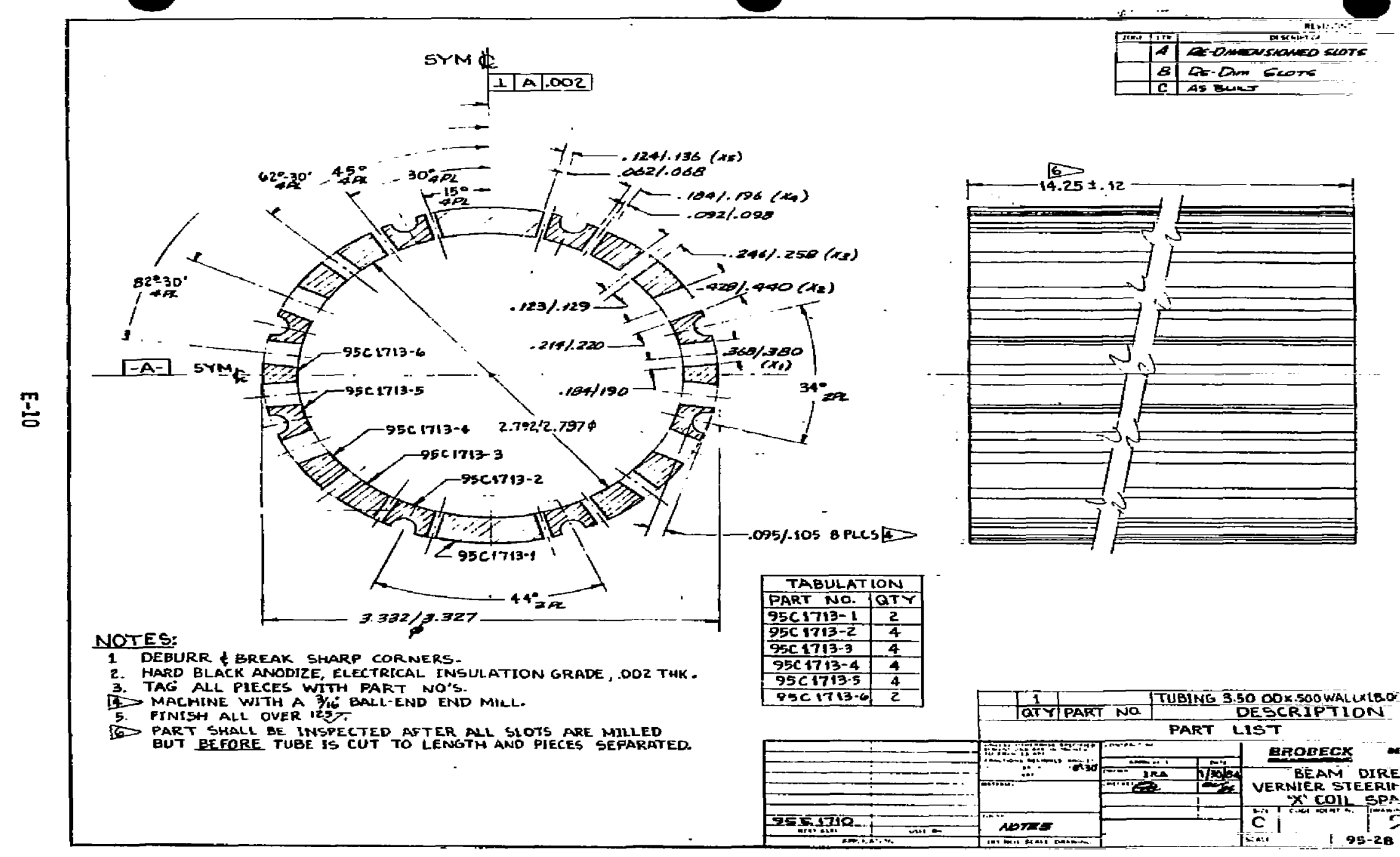




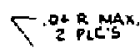

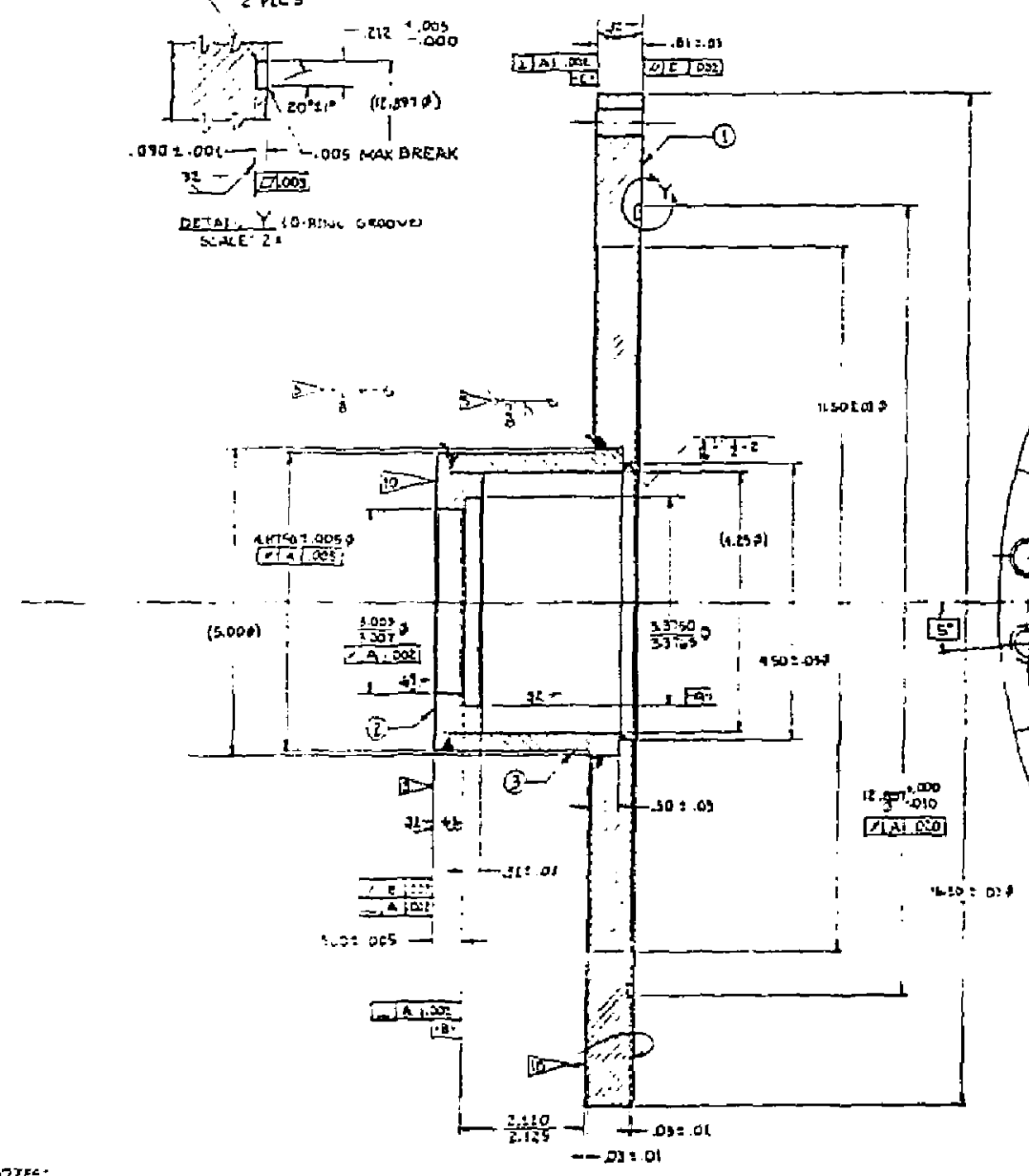

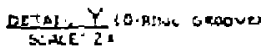

WOYE:

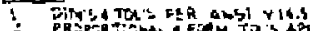

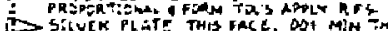

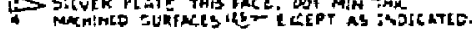

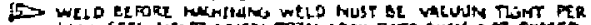

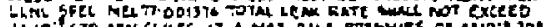

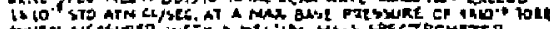

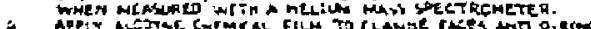

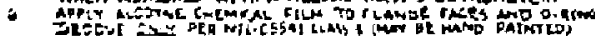

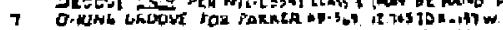

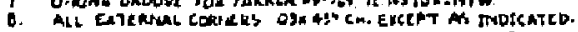

2. ALL INTIRNAL CORWR' .03R IXCEPT AS INDICATEZ

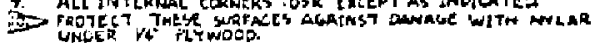




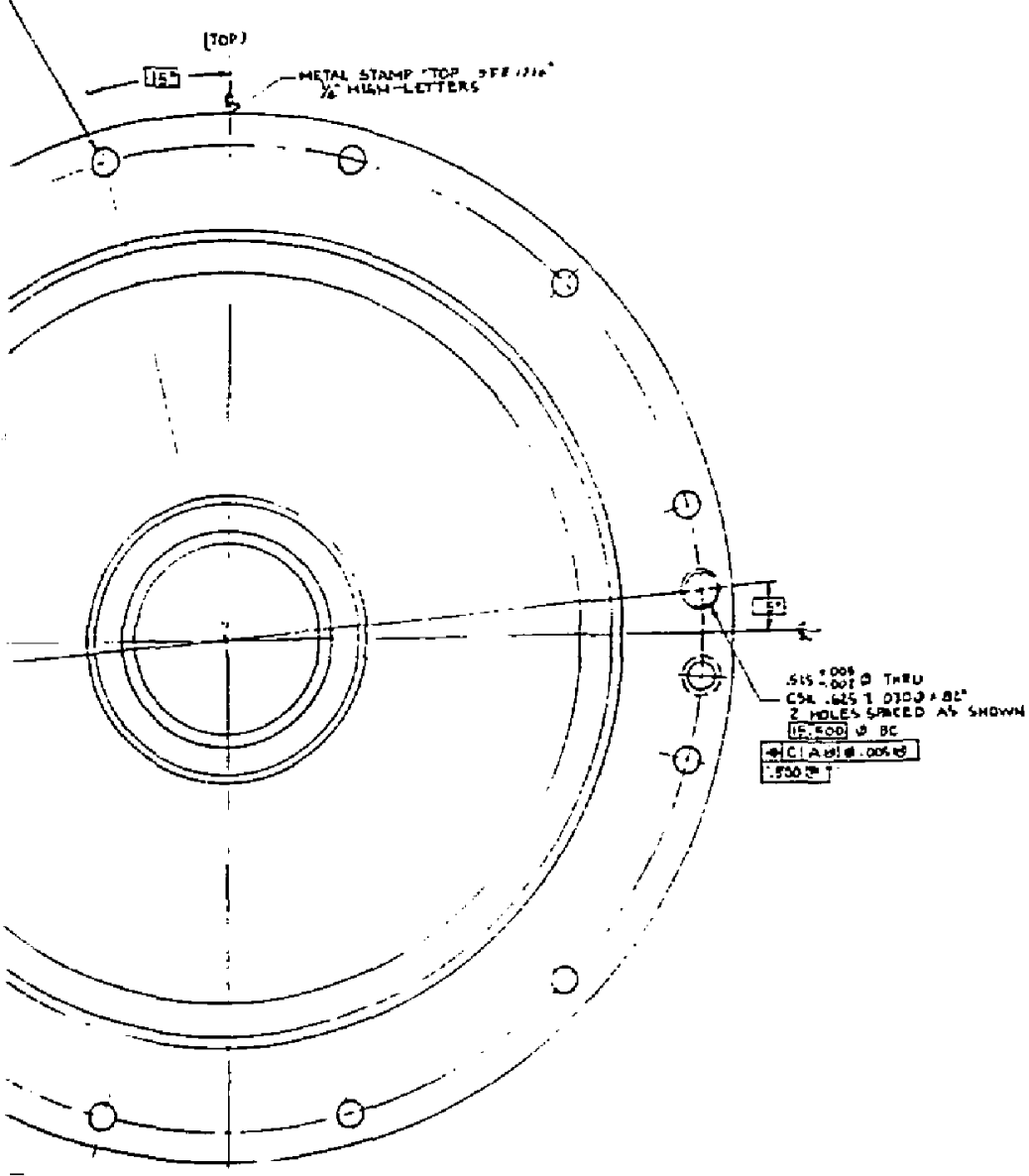

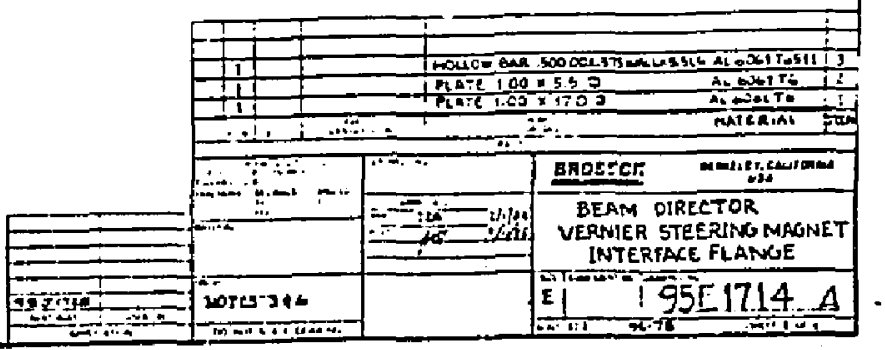



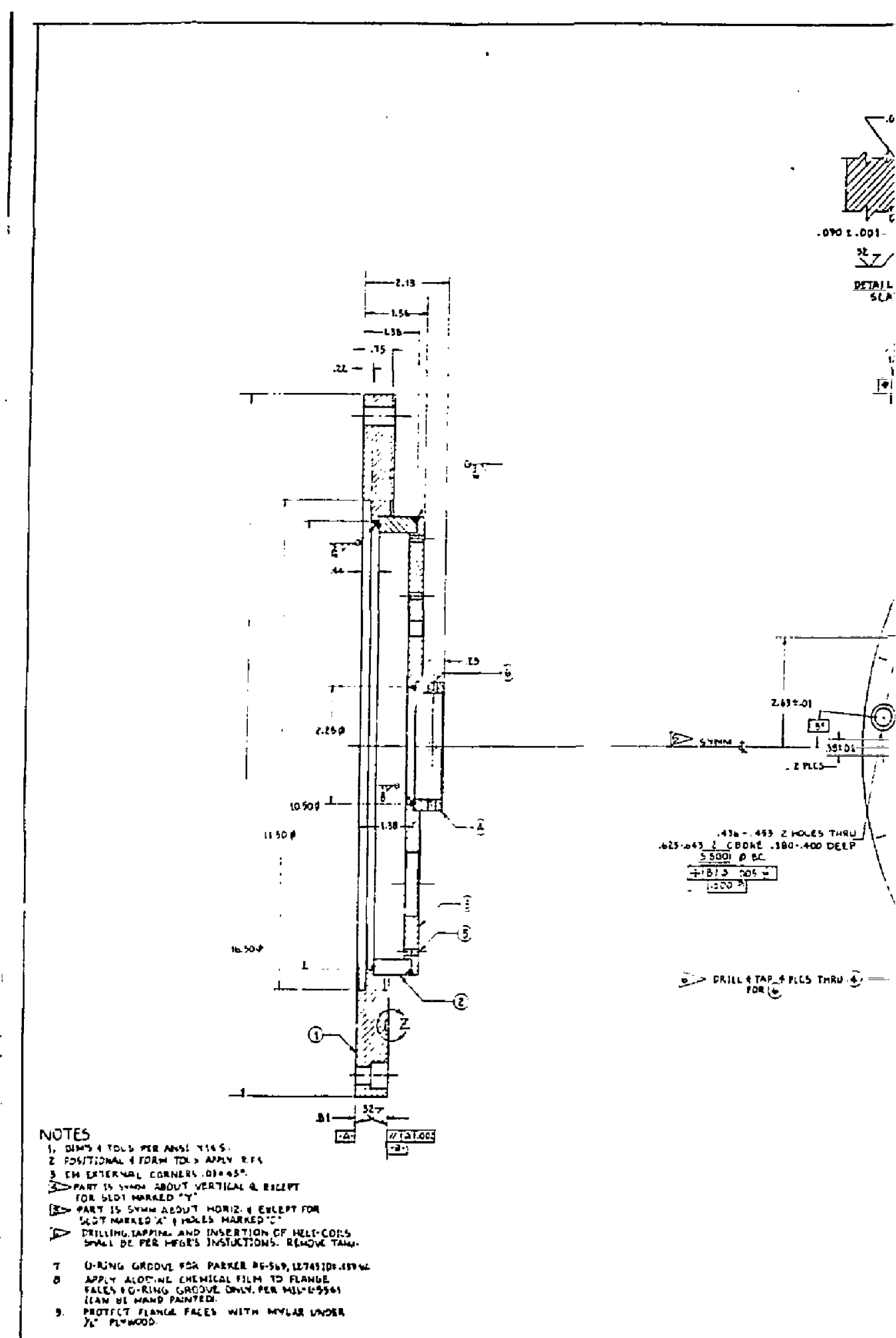

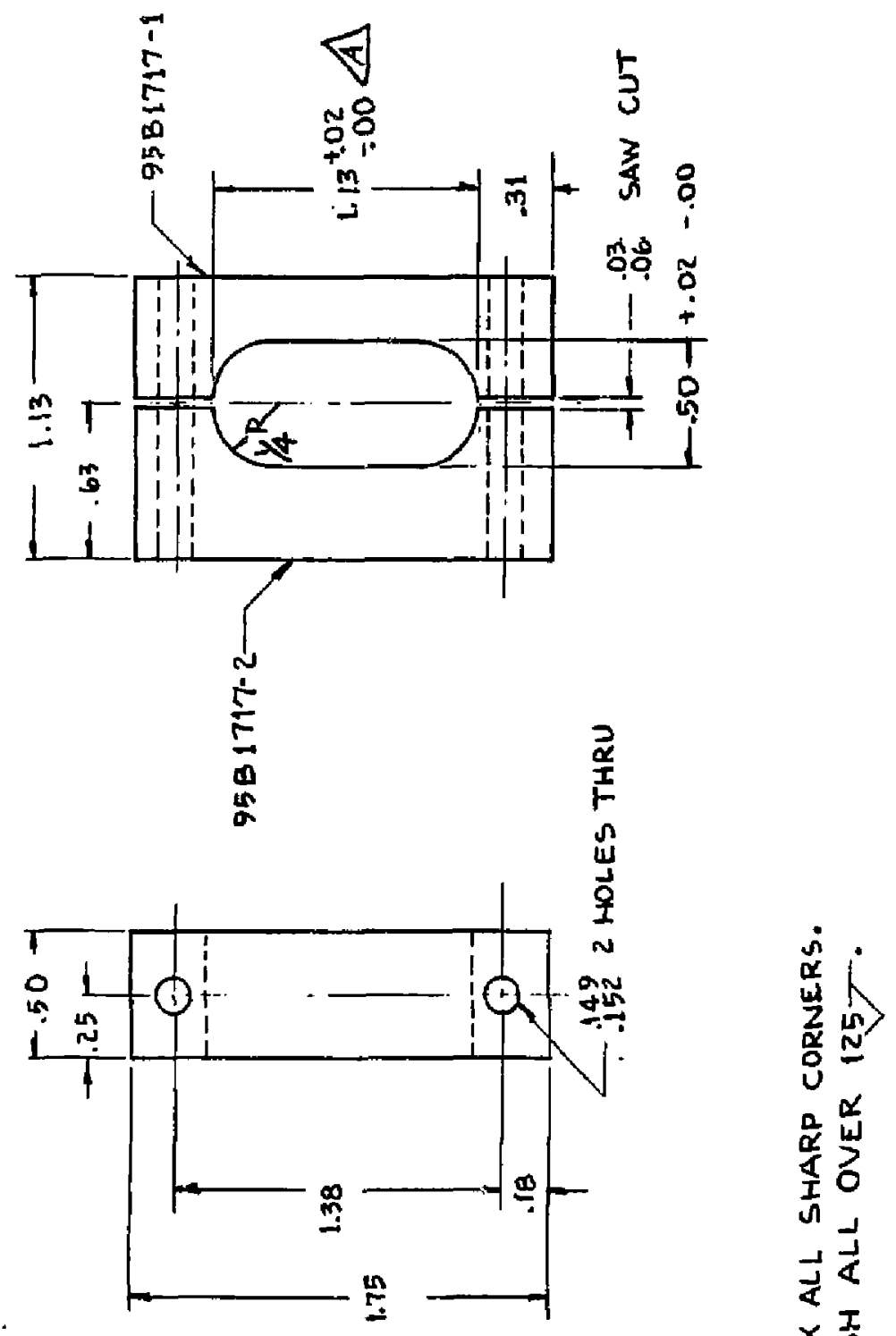

$\frac{2}{\alpha} \frac{\alpha}{x}$

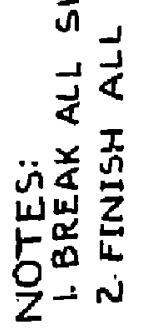




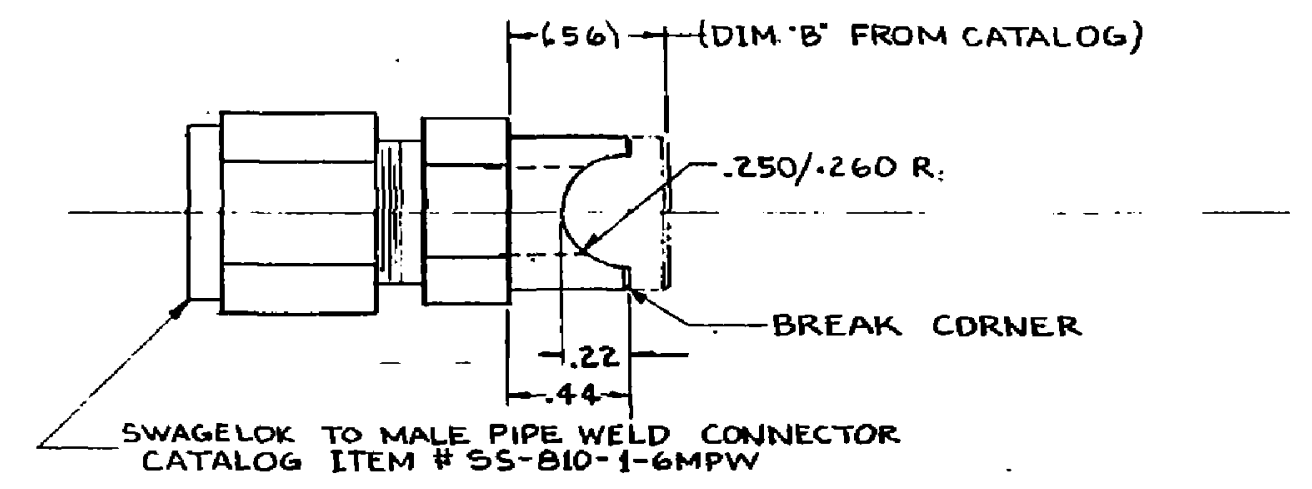

NOTE:

1. UNLESS OTHERWISE SPECIFIED, SURFACE

FINISH 125 .

\begin{tabular}{|c|c|c|c|c|c|c|}
\hline & & \multirow{2}{*}{ 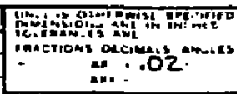 } & 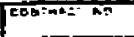 & & \multicolumn{2}{|c|}{ BPOEECK WENKELEYGALIFONNIA } \\
\hline & & & \multicolumn{2}{|c|}{\begin{tabular}{|c|c|} 
& rath \\
LRA & LT/EL \\
\end{tabular}} & \multirow{2}{*}{\multicolumn{2}{|c|}{$\begin{array}{l}\text { BEAM DRECTOR } \\
\text { VERNIER STEERING MAGNET } \\
\text { MANIFOLD FITTING - V/2 }\end{array}$}} \\
\hline & & STABLESS STL & cherces & $\cos$ & & \\
\hline $\begin{array}{l}9501721 \\
9501720\end{array}$ & & & & & Sige & 9581718 \\
\hline & Trom & 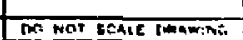 & & & SEA:I $2: 1$ & 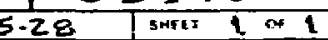 \\
\hline
\end{tabular}



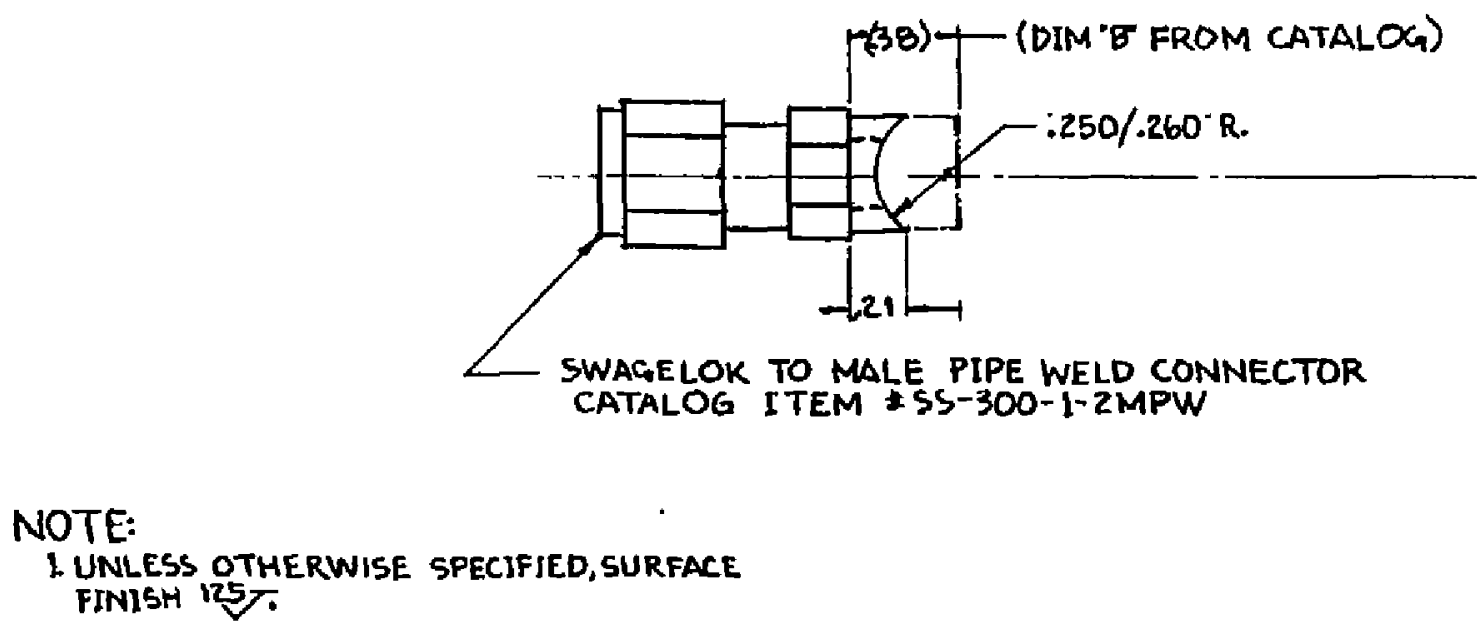

1. UNLESS OTHERWISE SPECIFIED, SURFACE FINISH $125 \%$

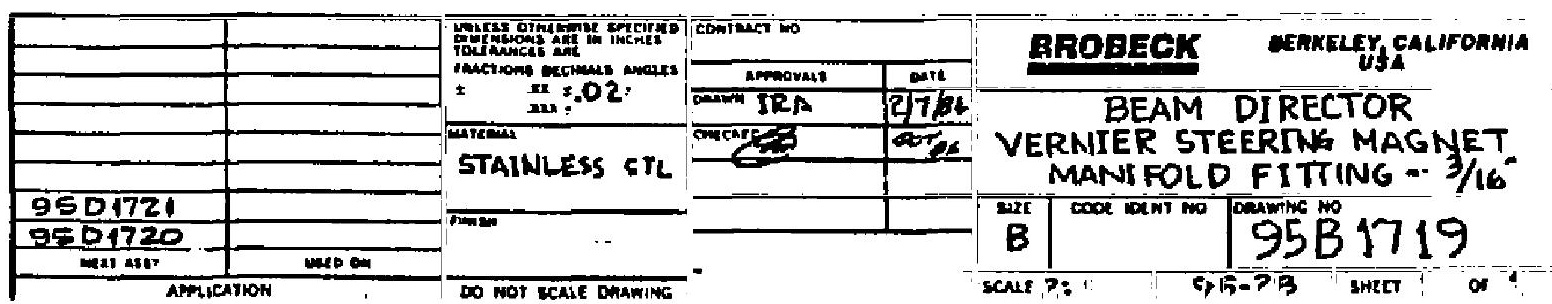


$V$

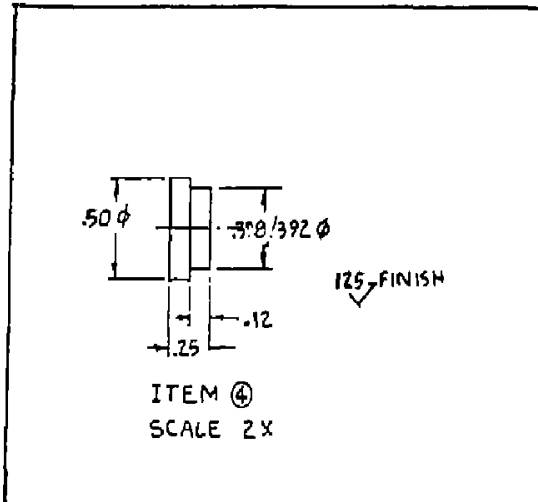

\section{1}


$V^{-<T 6}$

140

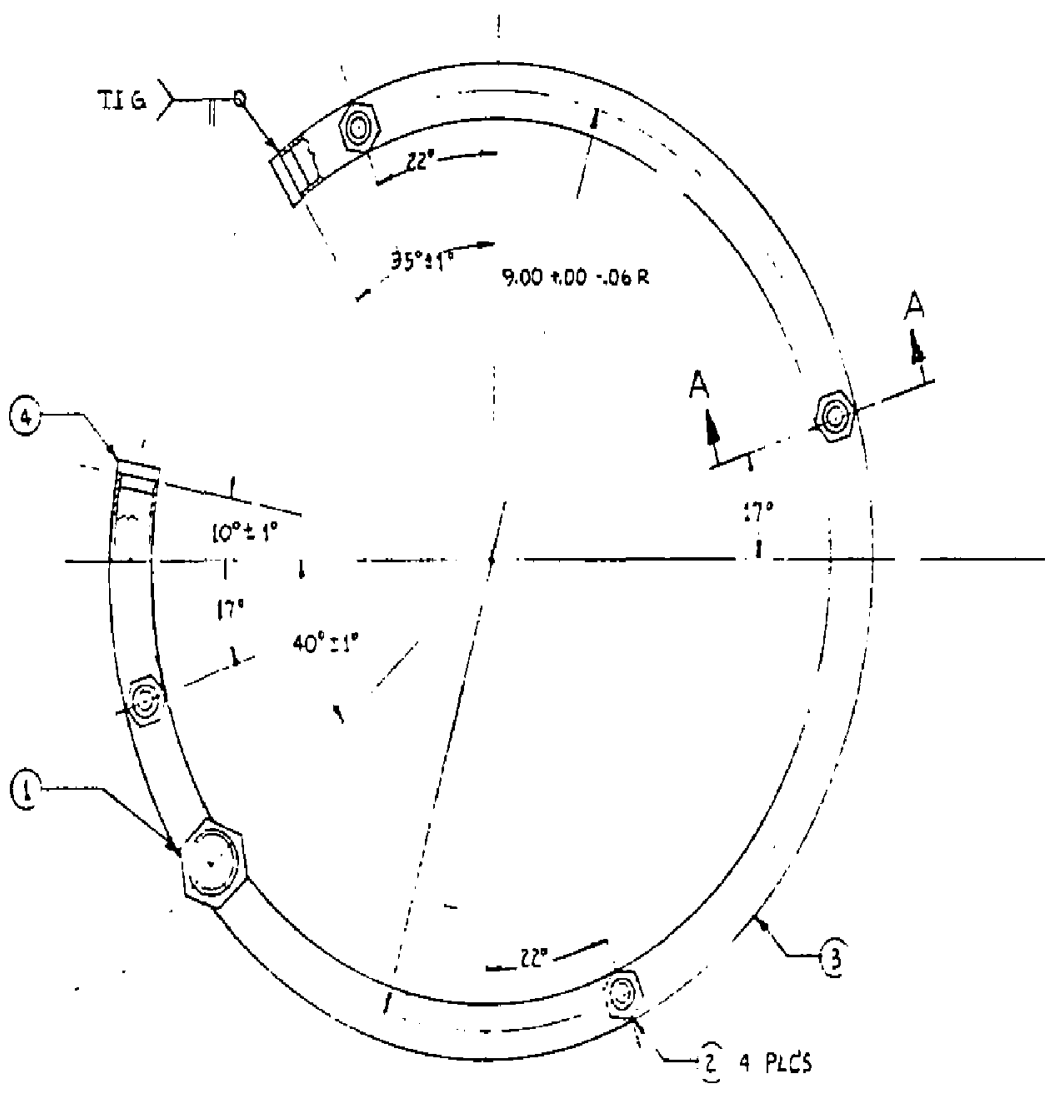

PAGE

E-16

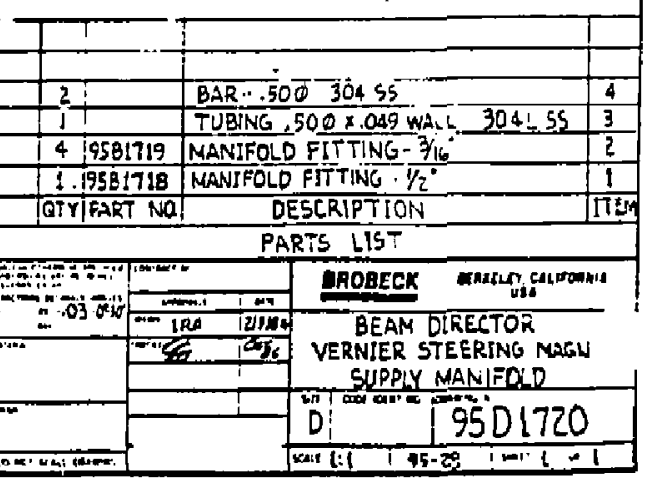




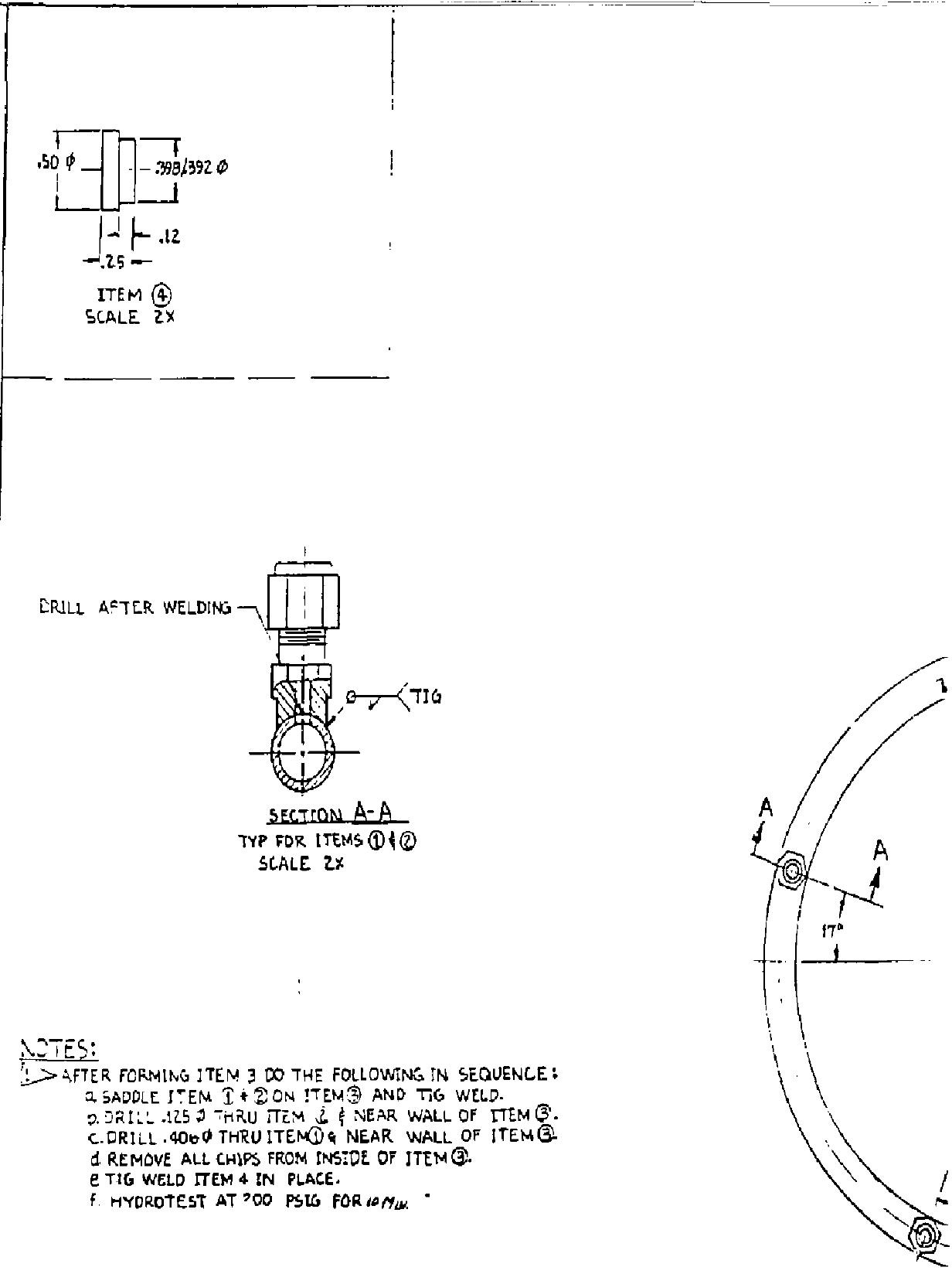



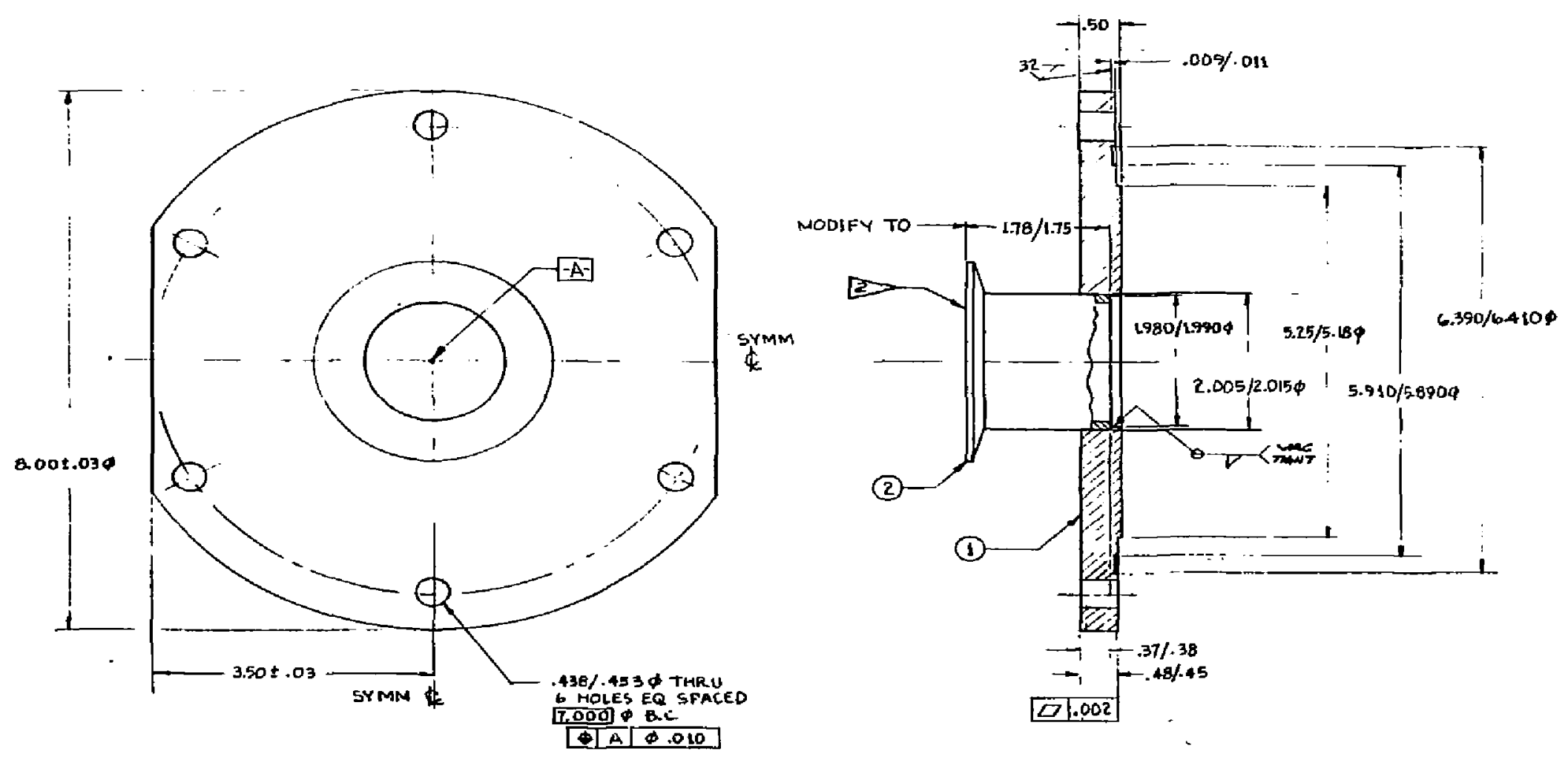

\section{NOTE:}

1. UNLESS OTHERWISE SPECIFIED SUREACE FIMISH 125

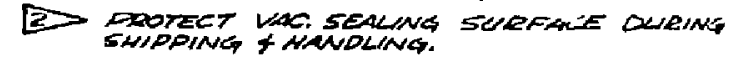

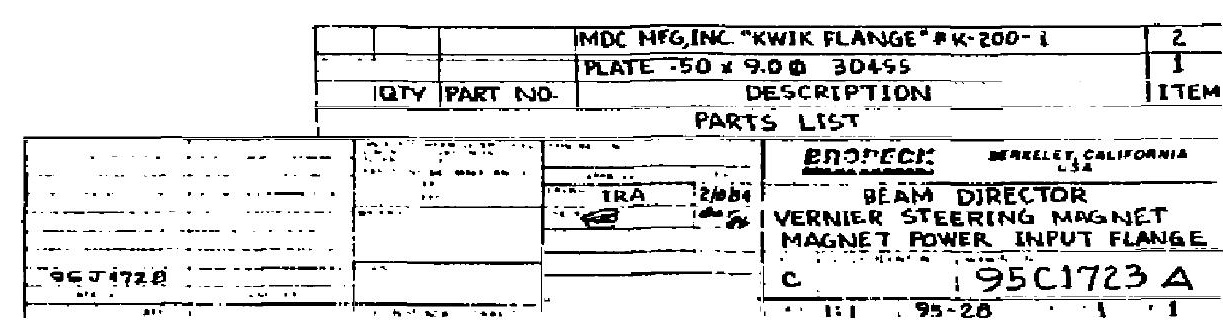



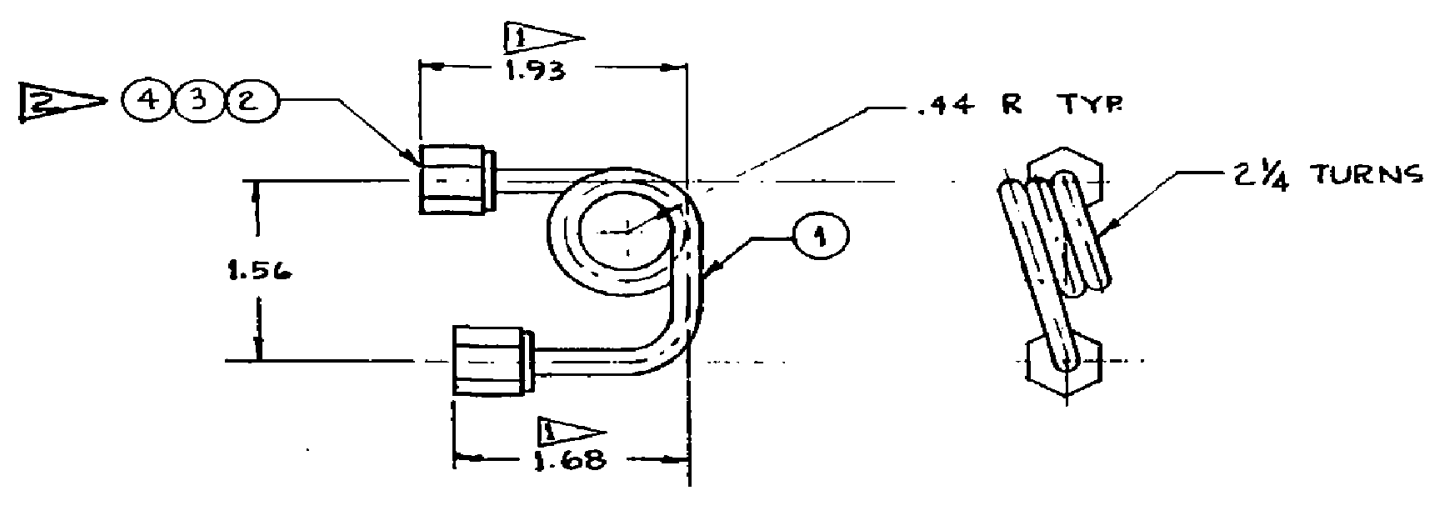

NOTE:

IS THESE DIMENSIONS ARE WITH NUTS AGATNST

SWNAGED-ON FERRULES.

2. ITEMS 3 4 ARE INSTALLED PER MFGR'S INSTRUCTIDNS USING A SWAGELOK PRE-5WAGING TOOL

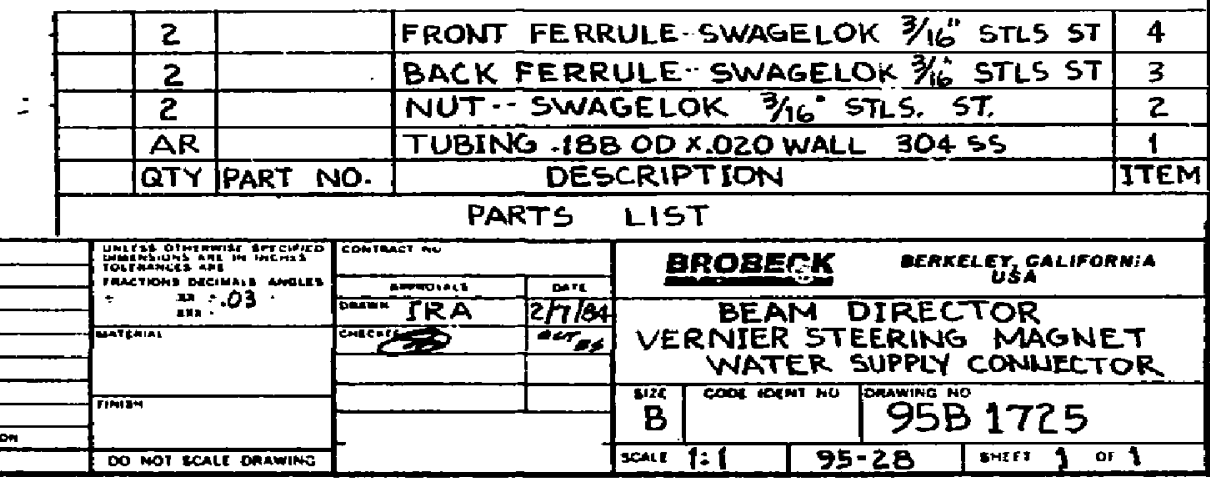




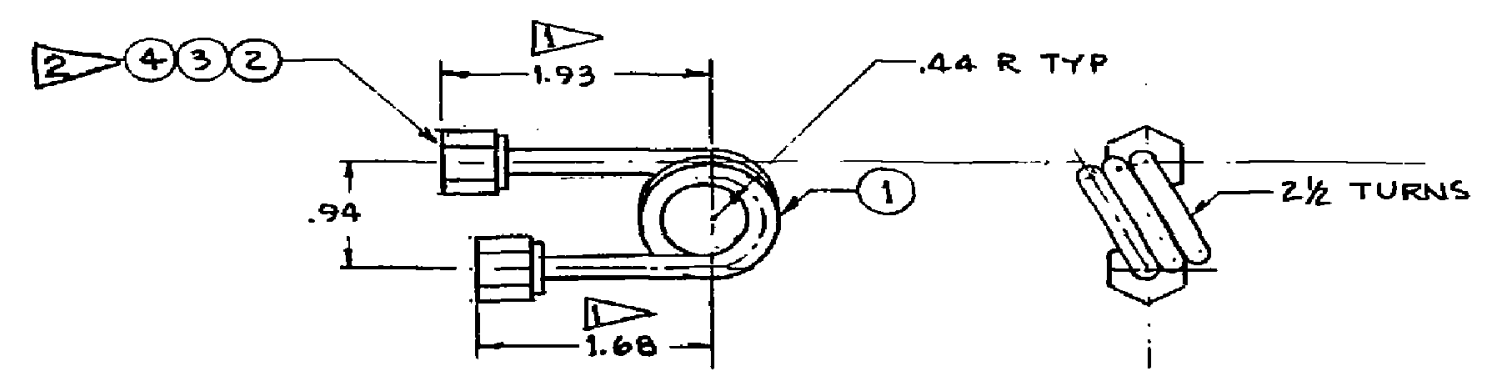

NOTE:

TI THESE DIMENSIONS ARE WITH NUTS AGAINST

SWAGED-ON FERRULES.

2. ITEMS $3 \$ 4$ ARE INSTALLED PER MFGR'S

INSTRUCTIONS USING A SWAGELOK

PRE-SWAGING TOOL.

\begin{tabular}{|c|c|c|c|}
\hline 2 & & FRONT FERRULE-SWAGELOK $3 / 6$ STLS 5 & 4 \\
\hline 2 & & BACK FERRULE-SWAGELOK 3/17 STLS & 3 \\
\hline 2 & & NUT--SWAGELOK $3 / 16^{\circ}$ STLS. ST & 2 \\
\hline AR & & TUBING . 188 OD $\times .020$ WALL 3045 & 1 \\
\hline QTY & PART NO. & DESCRIPTION & ITEM \\
\hline
\end{tabular}

\begin{tabular}{|c|c|c|c|c|}
\hline & & 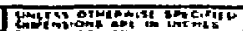 & 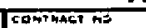 & \\
\hline & & 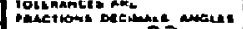 & & \\
\hline & & $13 \pm .03$ & DRAT IRA & 27764 \\
\hline & & कs & 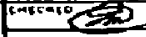 & $\infty$ \\
\hline & & & & \\
\hline 0551728 & & 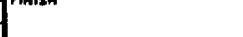 & & \\
\hline ANT & त्राOA & 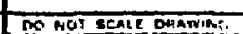 & & \\
\hline
\end{tabular}

BNDGECK

MERKELEY, GALIFONNIA

BEAM DIRECTOR

VERNIER STEERING MAGNET

WATER RETURN CONWETOR

B. COA HE WO $95 \mathrm{~B} 1726$

Aspricallo

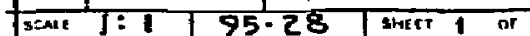




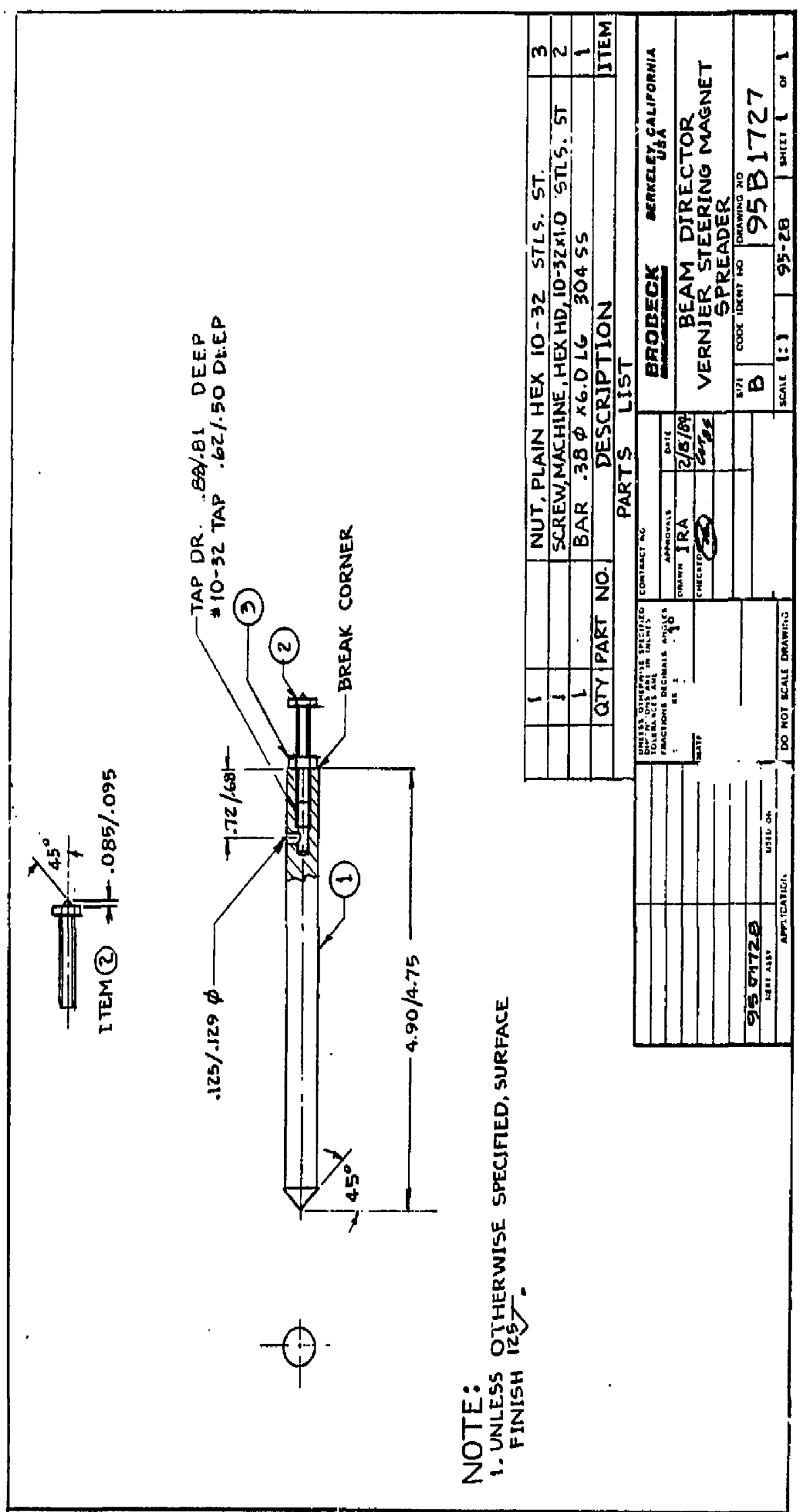



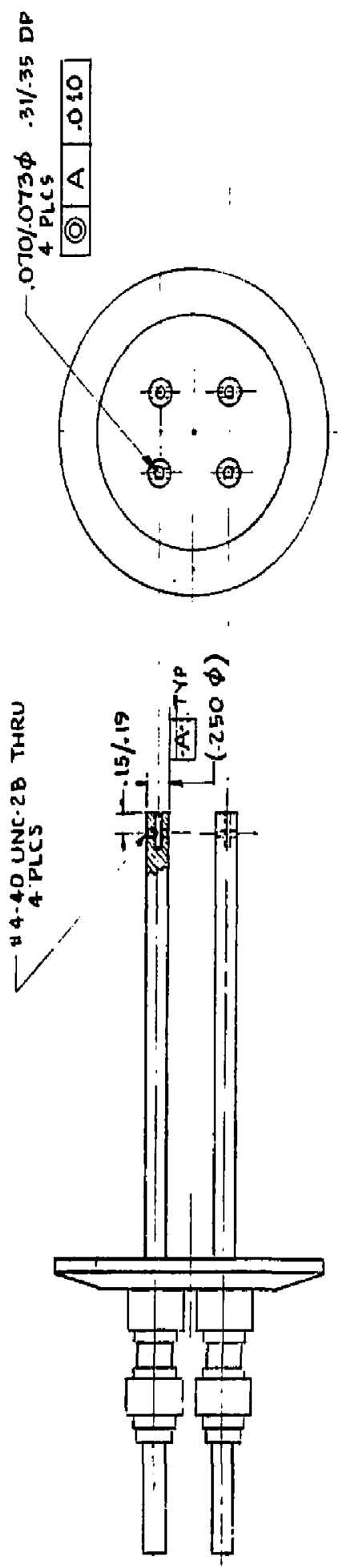

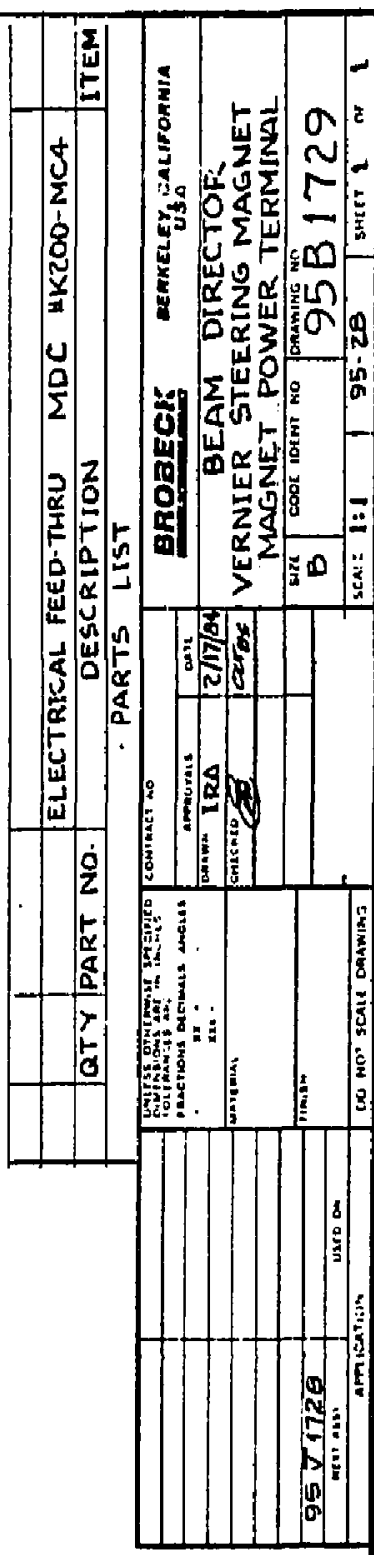



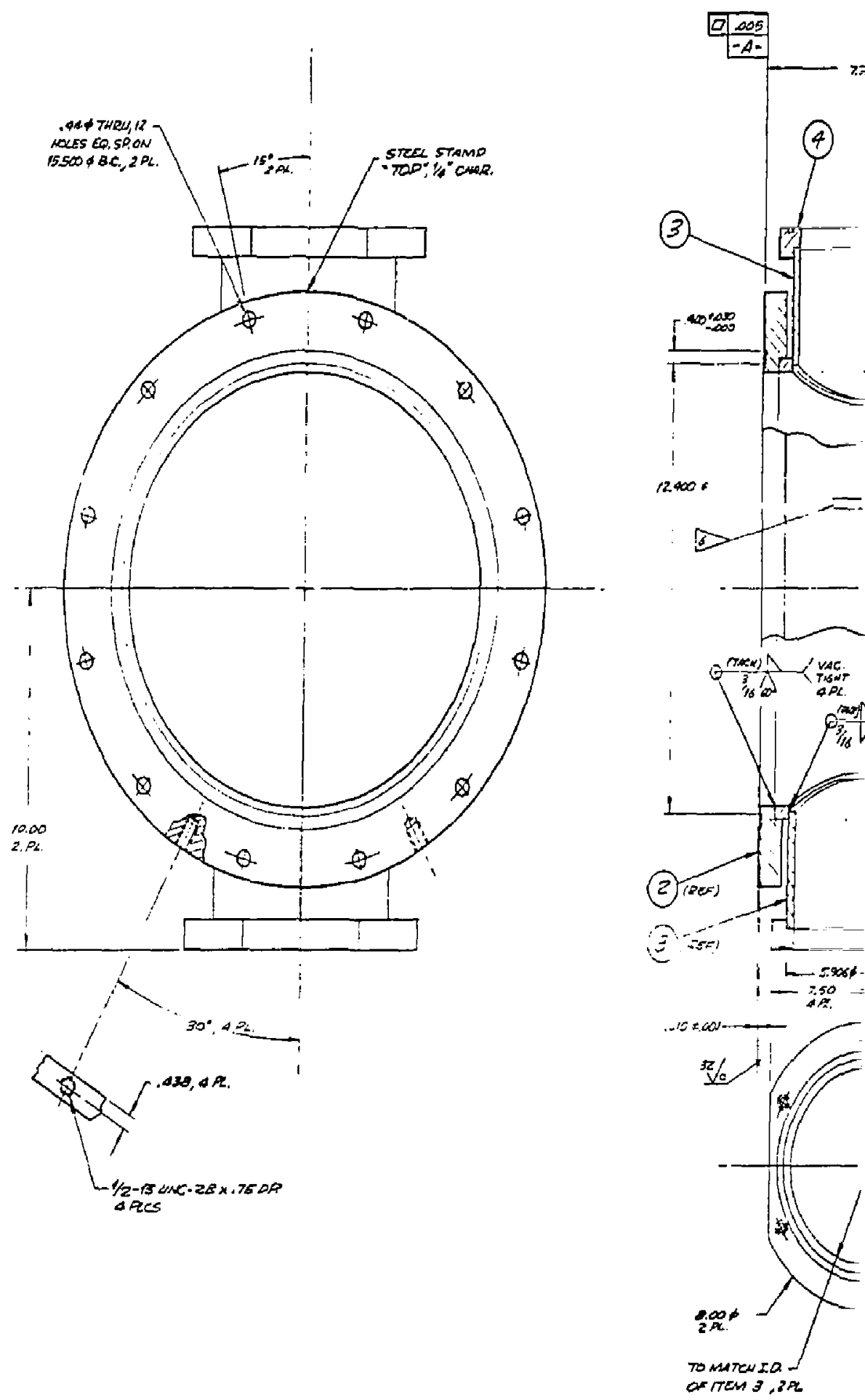

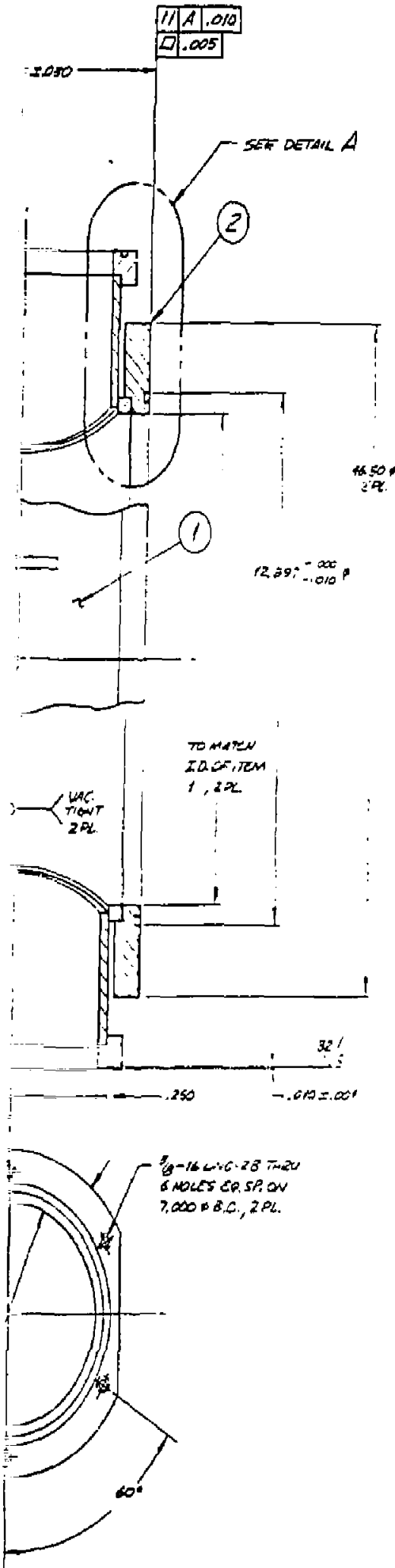

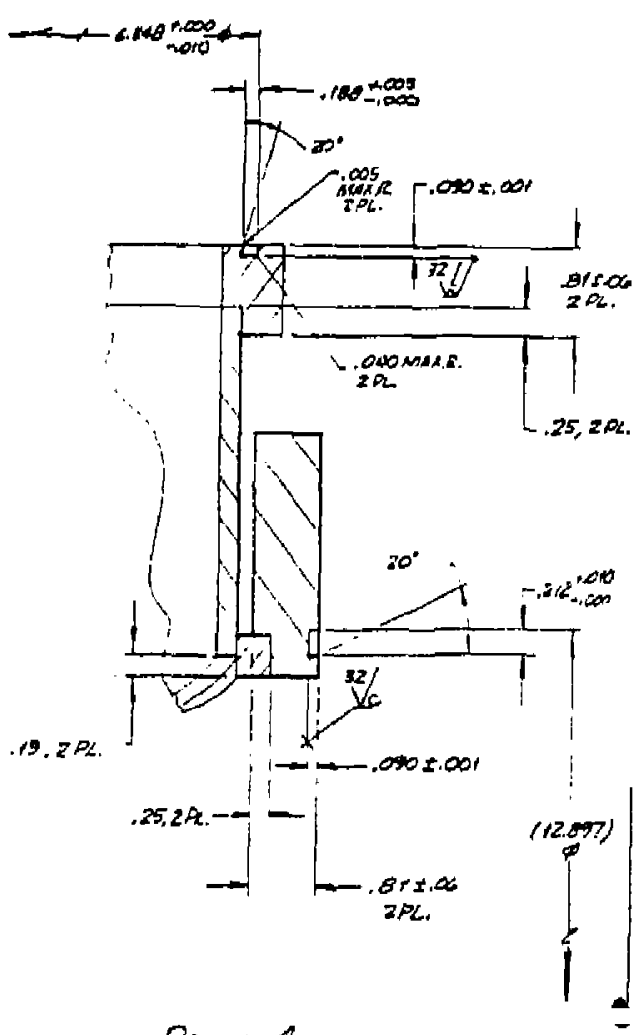

Notes:

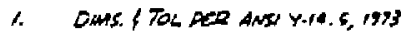

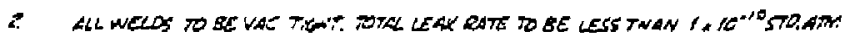

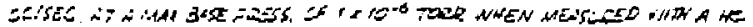

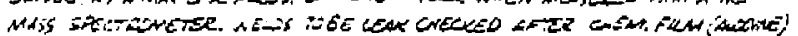

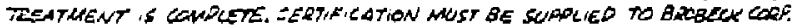

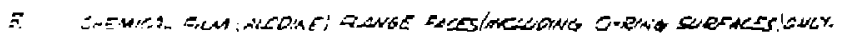

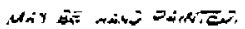

a SEN WPO: ILDPAT - DABUER 5.569

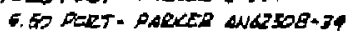

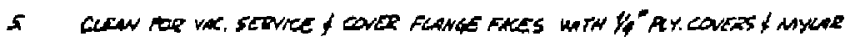

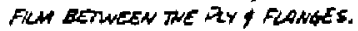

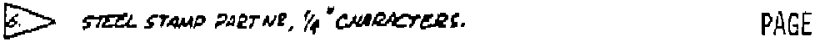

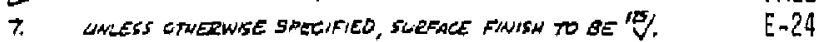

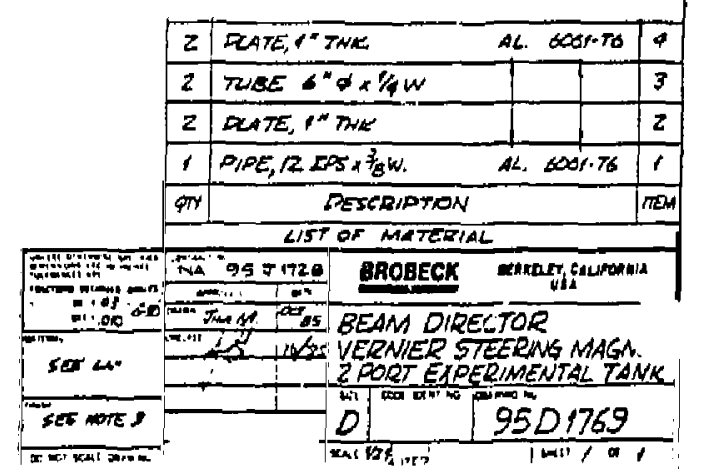




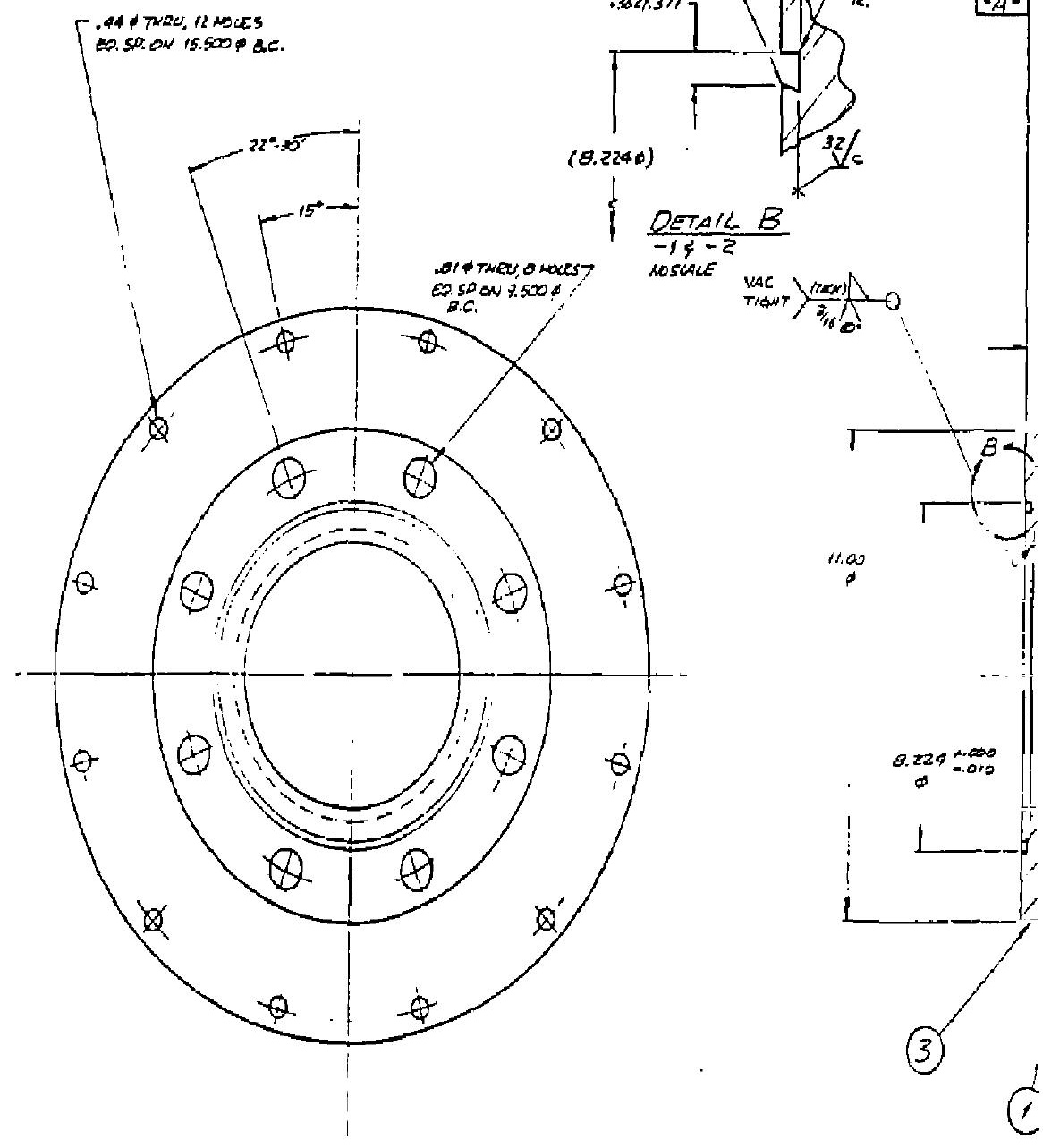

Notes:

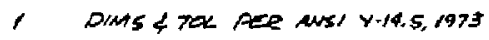

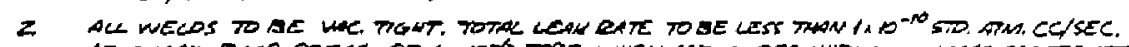

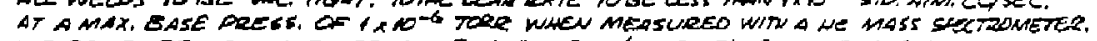

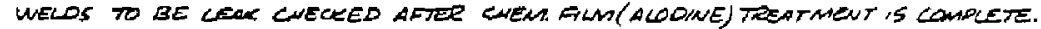
CERTHFTETION MUTT BE SUAPUEO TO GEBECK COEP.

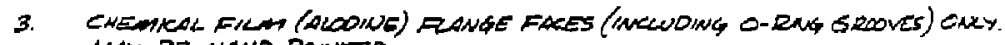
MAY BE HAND PANTED.

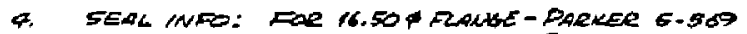

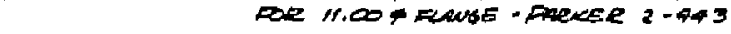

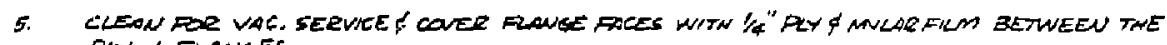
DLT I FLANGES.

QD STEEL STAMP PART NE L DASW NE, \%" CHORACTERS.

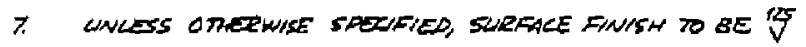



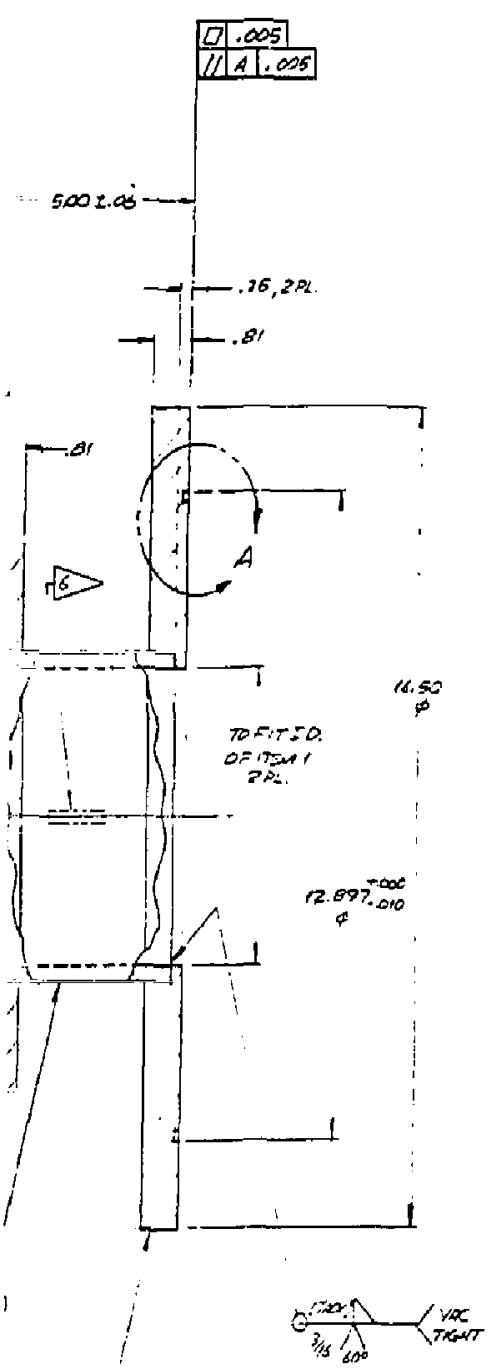

(E)

$-1 A 55 Y$
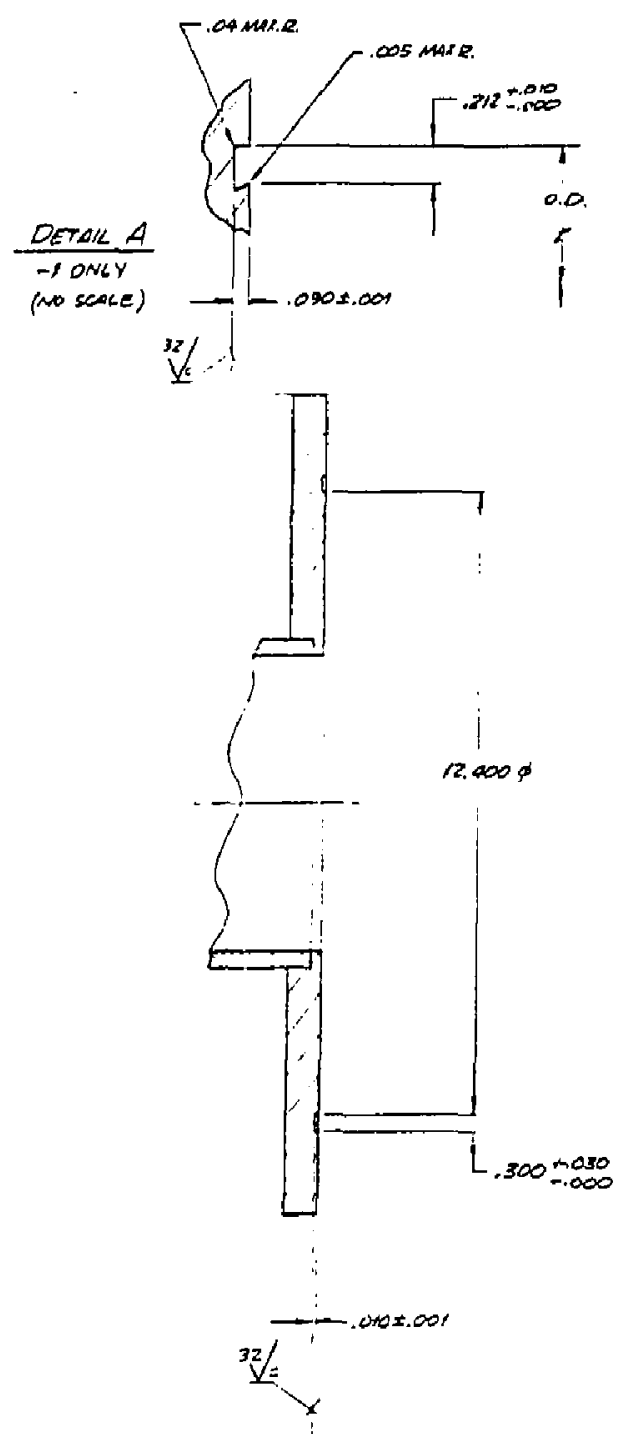

$-2455 \mathrm{Y}$

(ALC ONMS MOT SAOKW, SAME AS - I)

\begin{tabular}{|c|c|}
\hline \multicolumn{2}{|c|}{ TABLLATION } \\
\hline BACT NI & USE \\
\hline $9501771-1$ & LOSTREAM \\
\hline $950 N 71 \cdot Z$ & DOWNSTEEAM \\
\hline
\end{tabular}

PAGE

E-25

\begin{tabular}{|c|c|c|c|c|}
\hline 1 & \multicolumn{3}{|c|}{ DLATE, 1 THLL. AC. $0001 \% 70$} & 3 \\
\hline$t$ & \multicolumn{2}{|c|}{ ALTE. I'TUL. } & & $z$ \\
\hline 1 & \multicolumn{2}{|c|}{ PIPE A TAY SONE NO } & Alt.cods. TS & $r$ \\
\hline GN & \multicolumn{2}{|c|}{ DescaIPTION } & & 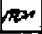 \\
\hline & \multicolumn{2}{|c|}{ CIFrar ANATERIAL } & & \\
\hline ma & $\frac{5571728}{-4+1}$ & BAOBECK & 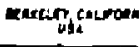 & \\
\hline
\end{tabular}

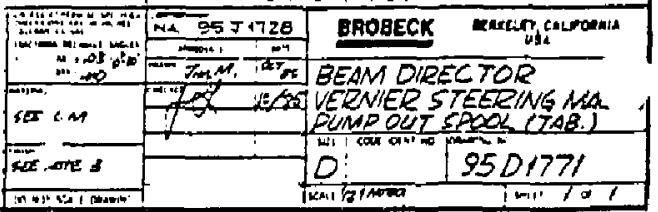




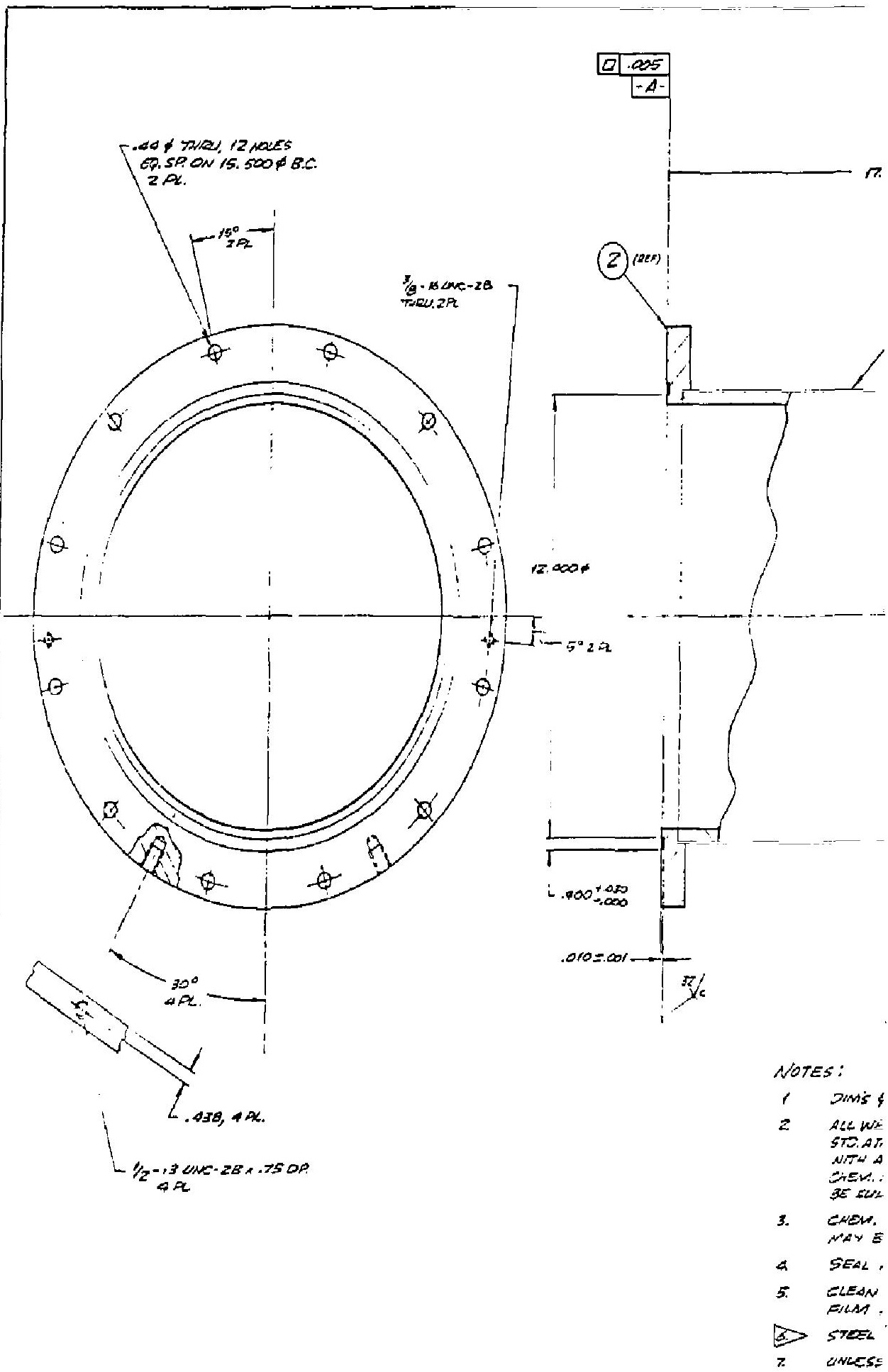



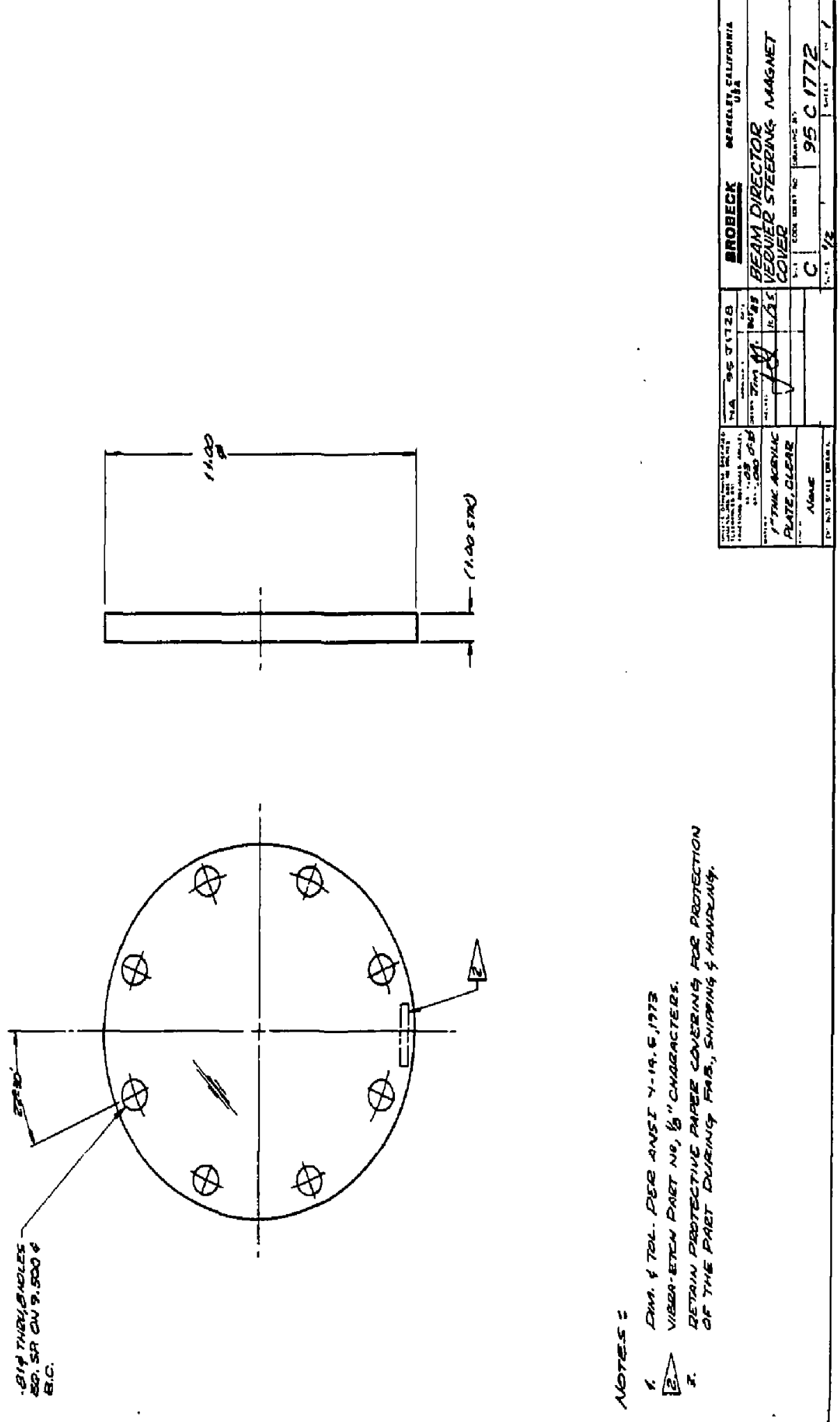


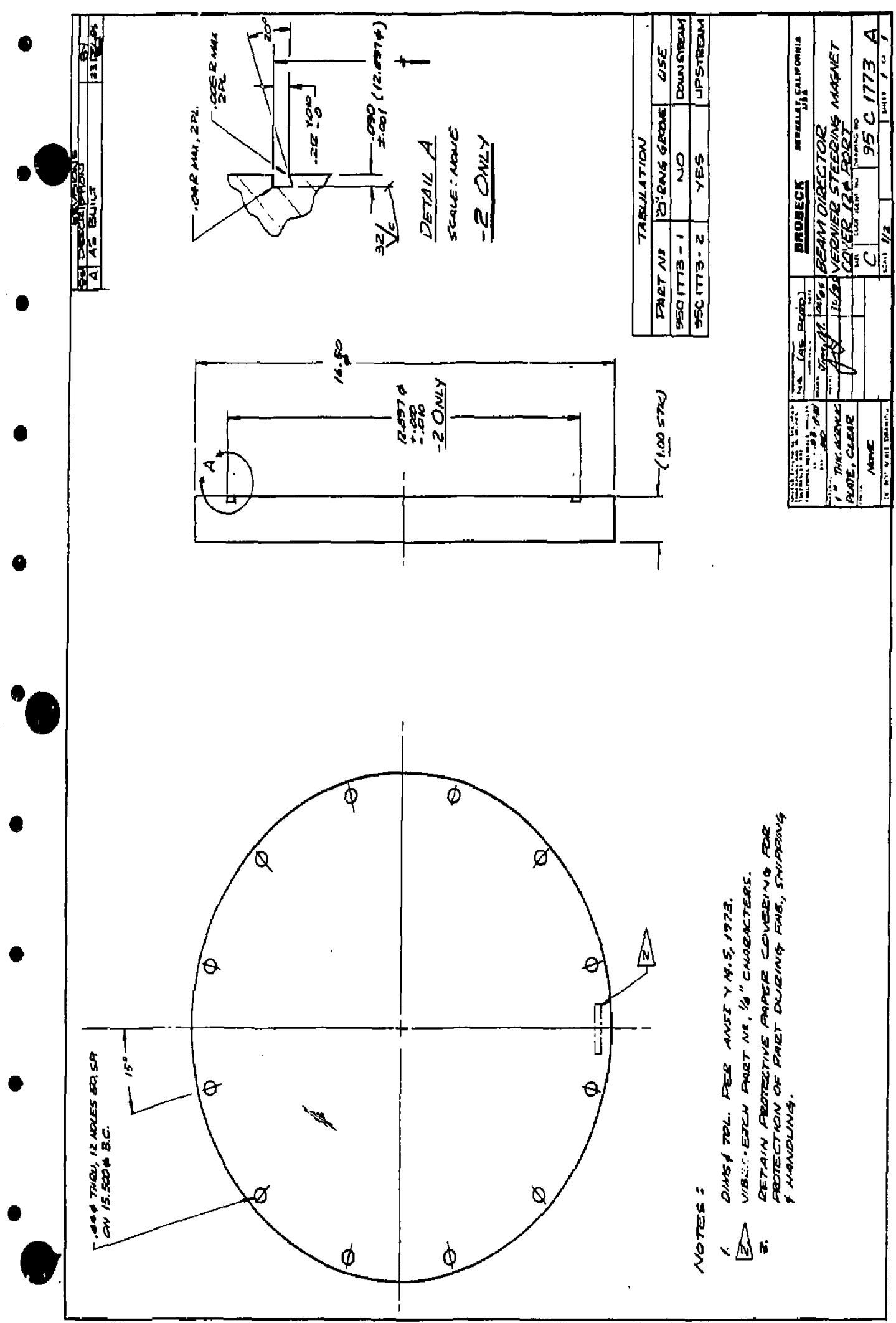




\section{APPENDIX F. MAGNETIC MEASUREMENTS REPORTS, LAWRENCE BERKELEY LABORATORY}

This appendix contains data provided by the Magnetic Measurements Engineering Group at Lawrence Berkeley Laboratory (LBL). Their report on the measurements of the individual magnet segments is included in its entirety. This is followed by excerpts from their reports on measurements of the quadrupole assemblies, dipole assemblies, and the vernier steering magnet. Full data reports are available from LBL upon request. 


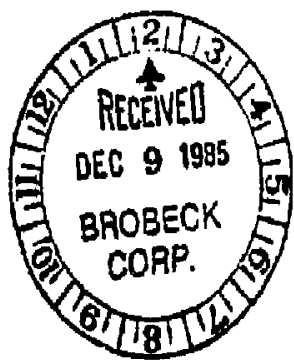

SUMMAFY OF MEASUREMENTS AND SORTING

QF

FERManent Magnets fuf the ata eEAM diseCtOF *

EY

DONALD H. NELSON \& DAVID A. VAN DYKE

NOVEMBER, 1985

LEL Electronics Engineering Note MT SE2

(LEID 1047?

This worl: was supported by the Director, Office of Energy Fiesearch, Office of High Energy and Nuclear Fhysics, High Energy Fhysics Division, U.S. Dept. of Energy, under Contract No. DE-ACOS-76SFO0O98. 


\section{INTFODUCTION}

The purpose of this Engineering Mote is to document messurements and sorting conductad by the LBL Magnetic Measurements Engineering Group. This worl: was undertaken to provide a basis for selectively distributing 367 permanent magnets among 17 beam-1 ine elements for the LLNL Advanced Test Accelerator (ATA) Beam Director. The measurements described in this report. fall into the following three categories:

I) Calibration measurements for the Magnetic-moment Measurement and Sorting System (MMSS),

II) Measurements for determining the accuracy and reproducibility of the MMSS, and

III) Measurements and sorting of $\$ 67$ ferrite magnets - Fesults. Measurements of strength and quality of the beam-line elements will be the subject of a separate repart.

\section{COOFDINATE SYSTEM}

To simplify descriptions of the MMSS we introduce two coordinate systems and define rotational error terms. Figure 1 represents a cross section of a pair of coils, coaxial on the z-axis and located symmetrically with respect to the origin of a cariesian coordinate system. We defire the angular eryors roll, pitch, and yaw as rotations about the $z$, $x$, snd $y$ axes $=s^{-}$ pectively - counter clockwise as viewed from the positive axis taward the origin. Figure 2 shows i serond cartesian coordinate system (primed) for a typical magnet tested. The origin of the primed coordinate system is at the centroid of the magnet. $y^{\prime}$ is normal to the large rectangular base formad by 82 and $e 1$; and $+y^{\prime}$ is directed as shown in figure 2 . The $z^{\prime}$ axis is paraliel to euge el. The $z^{\prime}$-axis is directed outward from the trapezoidally shaped end marked with an arrow signifying the easy axis tof magnetization). $+\because \cdot$ is defined sueh that $\vec{x}^{\prime} \times \vec{y}^{\prime}=\vec{z}^{\prime}, i$. e., such that the coordinate syst. 


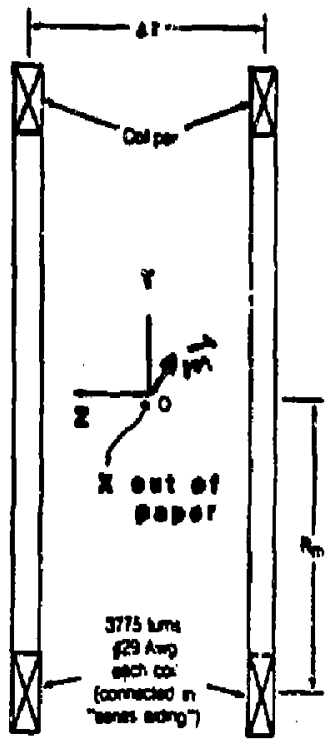

A mangotic dipole (in) at the origin of a

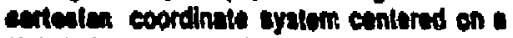
Matmholize coil palr; the axial separation (Az) equals the mean nodius of the coll bundie $\left(R_{m}\right)$

FIGURE 2.

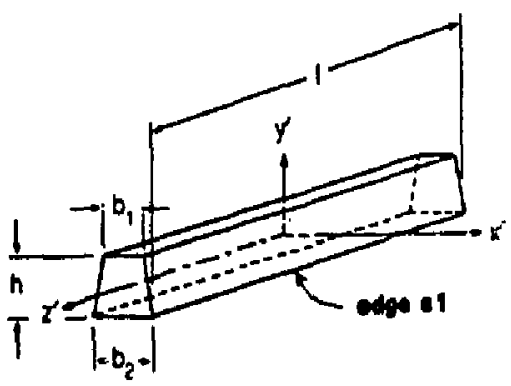

Aopresentative magnet--ATA beam diroctor 
Page 4 of 19 .

I.

CALIEFATING THE MMSS

We have shown, for a pair of iduntical, coaxial coils whose common axis is defined as the z-axis of a cylindrical coordinate system with its arigin nidway between the two cails, that Equation 1 describes the equivalence of two ratios: 1) the ratio of axial magnetic moment at the origin to magnetic flux linking the coils, and 2) the ratio of eurrent in the coils to the magnetic intensity at the origin.

$$
\begin{aligned}
m_{2}(0,0,0) / \psi_{\left(m_{\varepsilon}\right)} & =1 / H_{2}(0,0,0,1)=\mu_{1} I / E_{2}(0,0,0, I) \\
& =R_{m}^{*}\left(1+z_{0}^{2} / F_{m}^{2}\right)^{J / 2} n \text { [meters] }
\end{aligned}
$$

where:

$$
\begin{aligned}
& \mathrm{m}_{z}(0,0,0) \quad=(a \times i a l, z \text {, component of }) \text { magnetic moment at the origin [Wb:m] }
\end{aligned}
$$

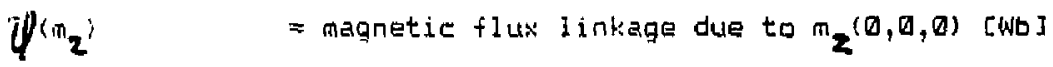

$$
\begin{aligned}
& \text { I } \quad=\text { zurrent flowing in each coil [A] } \\
& \left.\mathrm{H}_{2}(0,0,0, I) \quad=(a) i a l, 2, \text { component of }\right) \text { magnetic intensity at the origin } \\
& \text { due to } \mathrm{I}[\mathrm{A} / \mathrm{m}] \\
& \mathrm{B}_{\mathbf{Z}}(\theta, 0,0, I)=\mu_{0} \mathrm{H}_{\mathbf{Z}}(\theta, 0,0, I)=\text { magnetic induction corresponding to } \mathrm{H} \\
& \text { [T] } \\
& =\text { permeability of frae space }=4 \pi_{10}^{-7} \text { [Henries/m] } \\
& =\text { number of turns in each cail [dimensionless] } \\
& \text { = mean radius of the identical cails [m] } \\
& +f-z_{0} \quad=\text { lacations of coils [m] }
\end{aligned}
$$

The last expression in equation 1 dascribes the geametry of the coanial coil pair. For Helminoltz geometry $z_{0}=R_{m} / 2$, so $\left(1+z_{0}^{2} / F_{m}^{2}\right)^{2 / t}=1.398$ 
We Ealibrated the sensitivity of the MMSs coil pair to magnetic moment at the origin by detarmining the ratio $\left(I / H_{2}(0,0,0,1)\right.$. Fideiras $I$ and 4 illustrate the prineiplas involved in the derivation of Eq. I. Tabla I describes the equipment used. We measured current with an accuracy of 0.1\% by means of the current monitoring shunt. We measured $\theta_{2}(0,0,0, I)$ with the search-cul and the e! getronic integrator. The coillintagratar conbination was calibrated with the Flux-Standard.

In preparation for calibrating the MMSS we verified the ratio of flux-linliage produced by the fluk-standard to the turns area of the search

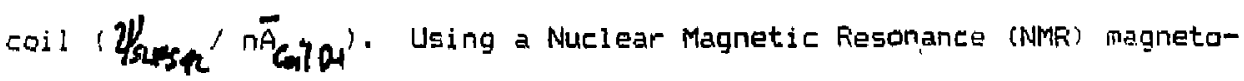
meter as a standard for magnetic induction and the LEL standard reference

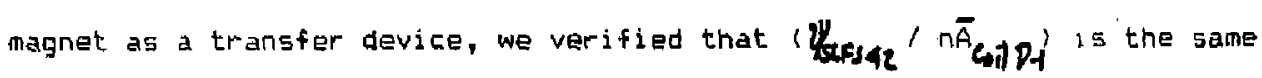
value ito $+1-0.2 \%$ as when it was first determined about 15 years ago.

We concluded that ur calibration of the MMSS was accurate to $0.2 \%$.

Table I Equipment Used For Calibrating the MMSS

Device

Search Coil

Flux Standard

Integrator

[WM1

Fower Suppiy

Shunt

DUM2

NMR: Control Unit Nithi Ainplifier NMF: Frobe

Magnet "EIG EERTHA"

Miniscope
Description

LEL, $\Pi \bar{A}=11.92 \mathrm{~m}$

LEL Drwg. \# SV4944

Flux $=0.05499[V \sec ]$

LBL Drwg. No. 6V1763

$\mathrm{R}=46.4$ [KOhms], $C=0.1[\mathrm{MF}]$,

Attenuator $=10.0$

KeitMley Mad 177

Elec. Frad. Shop Mad 49585

Leeds Northrup Co.

Keithley Mad 177

LBL Drwg. No. 16V111F1

LEL Drwg. No.16V112P1

LBL Drwg. No. 26V113F1 Fi. 4

LEL Fieference Magnet (Fermanent:

$\begin{aligned} & E=6.245 \mathrm{kG} \\ & \text { NLS Fod. MS } 215\end{aligned}$
DUE \#, $S / N$ etc.

Dt

42.05

S/N 1

SiN I0.45

$A E C$ \#29

$S / N \quad 10444$

$5 / N 22$

S/N 27

S:N 175

S/N 1973 


\section{FIGURE 3.}

\section{POWERED COILS DERIVATION OF $\vec{H}_{z}(I)$}

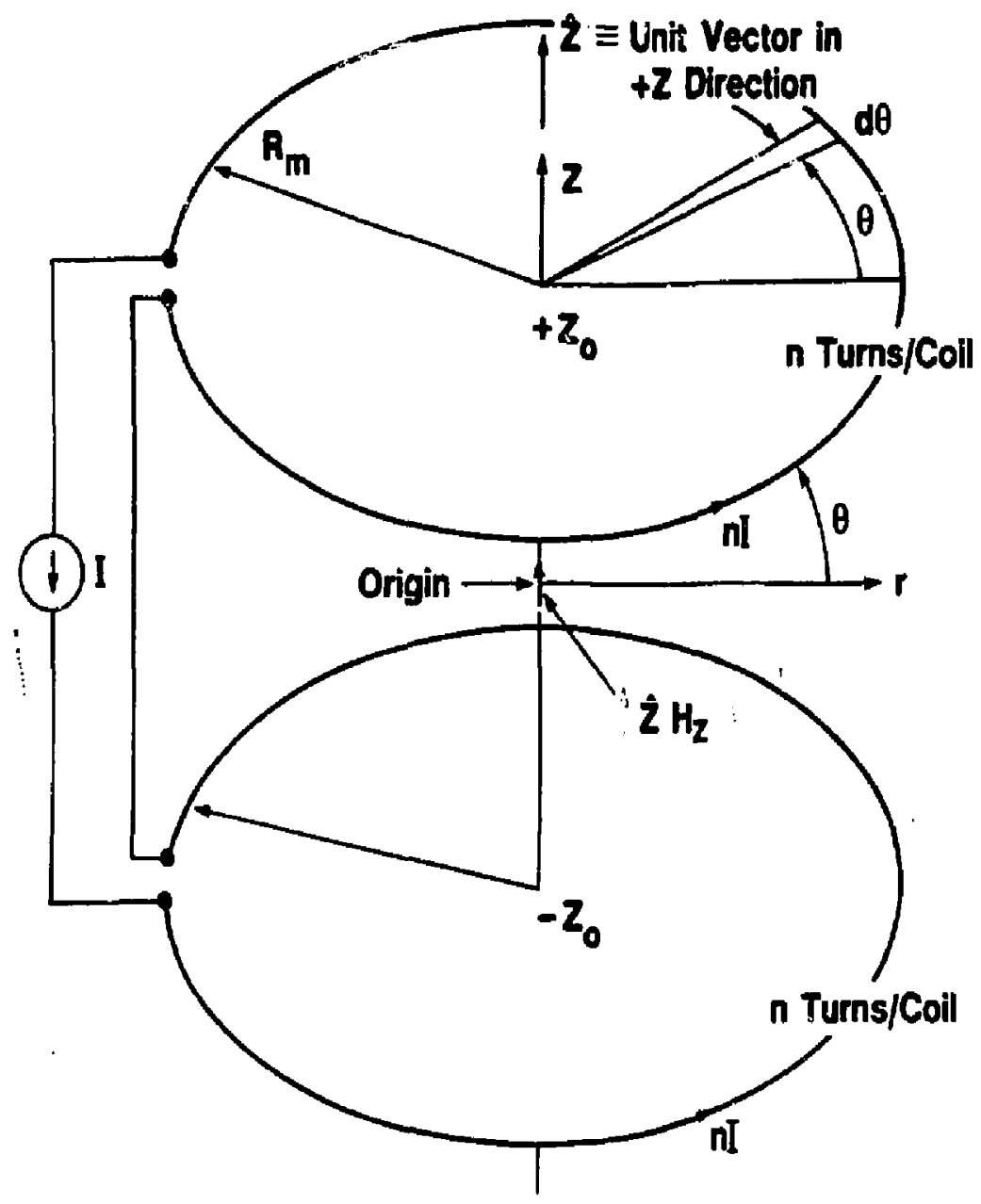

$$
\begin{aligned}
H_{Z}(2 n l) & =\vec{H}(2 n I) \cdot \hat{Z} \\
& =2 n \int_{\theta=0}^{2 \pi} \frac{1 R_{m}^{2} d \theta}{4 \pi\left(R_{m}^{2}+Z_{0}^{2}\right)^{3 / 2}} \\
& =\frac{n I}{R_{m}\left(1+\left(Z_{0} / R_{m}\right)^{2}\right)^{3 / 2}}
\end{aligned}
$$




\section{FIGURE 4.}

\section{DETECTOR COILS DERIVATION OF $\psi(\vec{m})$}

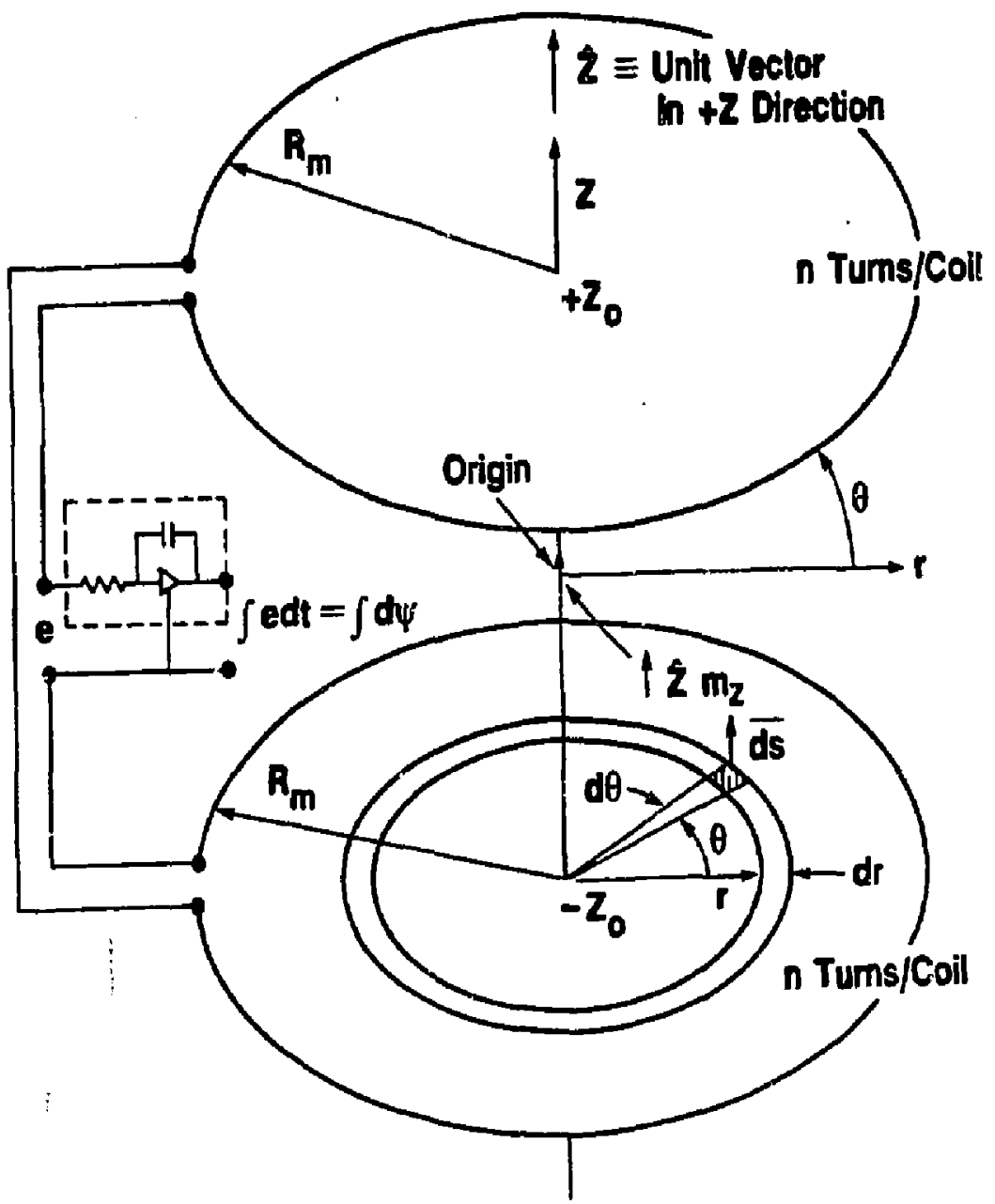

$$
\begin{aligned}
\psi\left(m_{z}(0,0,0)\right) & =2 n \emptyset \vec{B} \cdot \overrightarrow{d s} \\
& =2 n \int_{\theta=0}^{2 \pi} \int_{r=0}^{R_{m}} \frac{m_{z} \times\left(2 Z_{0}^{2}-r^{2}\right)^{*}}{4\left(r^{2}+Z_{0}^{2}\right)^{5 / 2}} d r d \theta \\
& =\frac{n m_{Z}}{R_{m}\left(1+\left(Z_{0} / R_{m}\right)^{2}\right)^{3 / 2}}
\end{aligned}
$$

- Dols, Nelson, "Point Dipole Approximation to the Magnetic Fiedd of a Solenoid" LBL Eng. Nole MT 253 (1976), Eq. 1. 
Tu fessure the accuracy and raprooducibility of the MMSS, we made

(in addition to the calibration measurements diseussed previously) the following tasts:

1. aro rosoiution tests - measurements of the noise lavel of a complete masurement sequence without a magnet installed in the positioning fisture,

2. coproducibility tests - comparisons of repeated measurements of three componerits of magnetic moment of the same magnets, 3. translation tests - measurements of the three components with the magnet translated $S \mathrm{~cm}$ along the $x, y$, or $z$ axes, and

4. rotation tests - measurements of the three components with the magnetat the origin, but rotated 100 milliradians about the $x, y$, or $z$ aisis.

The zero resolution tests were made without a magnet in the positioning fixture. The reproducibility tests were conducted with two magnets, one with its easy axis (of magnetization) along the $x$-axis (s/n 105013) and tne otharwith its easy axis along the $y-a x i s$ (s/n 103003). The transiation tests wer conducted with magnet $s / n$ 10300s. The rotation tests were conducted with magnet $5 / 7405253$. The results of these tests are represented in Tables II - V.

since magnets have been reworked, i.e. , demagnetized, ground to fit into sidj-issembiles. and remagreatized, the magnitudes of magnetic moillent: aria rot of interest. For this rason we present the test results in te ns of percent of mo' cthe magnetic mament amplitude in the $x^{\prime} y$ ' plane. For the reader's convenience we define terms used in Tables II - V below:

$(x, y, z)$ coordinates of Helmholtz coil-pair

roli rotation about the z-axis

pitch rotatian about the $x$-axis

yan rotation about the $y$-anis

$i \quad y^{\prime}, y^{\prime}$, or $z^{\prime}$ (coordinate of) magnet

$m i \quad m, m y$ or $m_{z}^{\prime}$ (component of magnetic moment, [Maxwell cm])

$<.05$ less than $0.05 \%$ of $\left\langle m \times r=\sqrt{m^{3}+m^{2}}\right)$

Glossary of Terms used in Tabies If - $V$ 
Tatle It Sumnar; af Tzra Fogolution Taste

tmeasursmenta with no magnet in the positionine finture'

Quartity iacukated

18 Measurement. Average

18 Measurenent Standard Deviation

Maximum Deviation (from zero)
Magnitude [\% of (12000. Marwell cm) ]

0.05

0.05

$+1-0.07$

Table II I Standard Deviations of 10 Measurements of Each Quantity

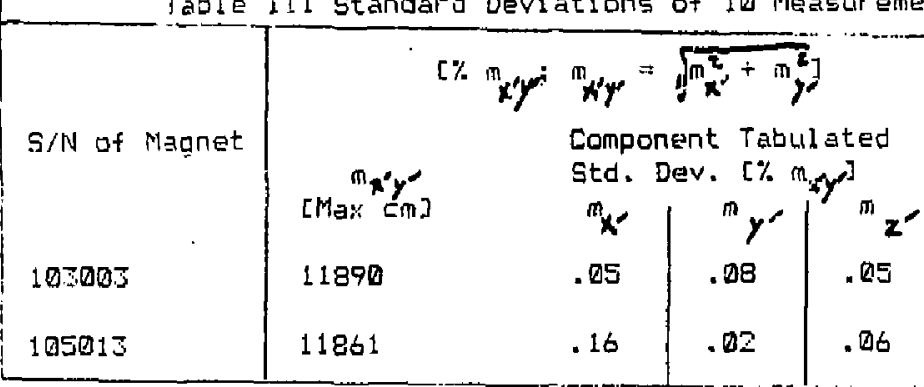

Table IV The measured effects of Translation Errors (S/N 103003)

(moment)

component (i)

measured

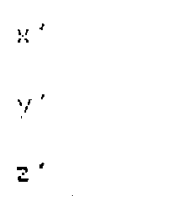

\begin{tabular}{|c|c|c|}
\hline$\frac{\text { delta } m i}{\text { delta }:}$ & 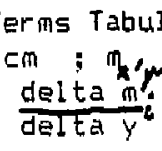 & 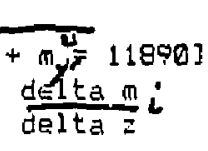 \\
\hline+0.01 & +0.01 & +0.02 \\
\hline+0.02 & 0.00 & +0.01 \\
\hline 0.00 & -0.02 & 0.00 \\
\hline
\end{tabular}

Table $V$ The Measured Effects of Rotational Errors (5/N 405253)

(moment)

i component (i) measured

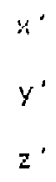

Errat- Terms Tabulated

$\left[\% m_{x<}, 100\right.$ ir ; $m_{k \prime}=\sqrt{m_{x^{\prime}}^{2}+m_{y}^{2}} 15274$ Na: Em] delta $m:$ delta ral deltami $\left.\right|_{\text {delta pitch }} \frac{\text { deltami }}{\text { delta yaw }}$

$<.05$

$<.05$ $\therefore .05$

$\measuredangle .05$

$-0.77$

$-0.62$

$<.05$ 
Test Equjpment

Figure 5 is a block diagram of the MMSS. Table VI lists specific equipmont: used for measuring 36? magriets for the ATA Eeam Director. Buth the data acquisition and sorting codes are seved on a total of 10 floppy djscs. Hard copies of directories (of) and source listings (for) programs and sutroutines on the floppy disce are filed, in LEL Engineering Date Fool: ME EOOL: H $6 ? 1-[$.

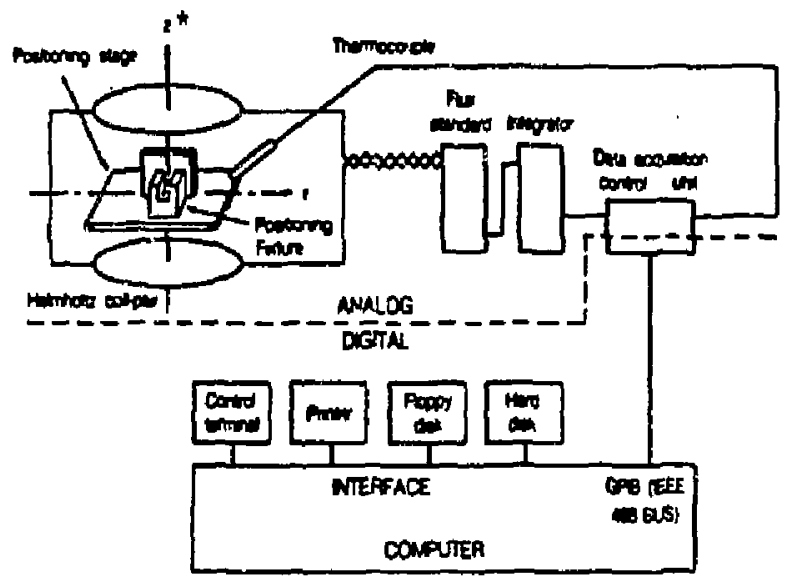

\section{FIGURE 5. Magnetic-mornomt maasurernent and sorting oystem (Muss) cetwenubie ditgram}

* Since Bevatron pulsing induced a significant vertical field at the location of the MSS, we rotated the coil pair $90^{\circ}$ so the $z$-axis was horizonta? as shown in Figure $l$.

Device

\section{Coil Fair}

Fositioning Stage

Fositioning Fisture

Flus: Standard

Integrator

Data Acquisition \&

Contral Unit

Computer
Tatle VI Equipment Li Lt

Description

DOE \#, S/A ETC

(Ea) $\$ 775$ turns \# 29 AWG, fin $=.363[\mathrm{~m}]$ None

Lel Frecision, Special Furpose None

LEL Frecision Cute 5":5"35" Aluminum Norie

LEL Drwg. \# SV4994, Flu: $=.05499$ [V sec] S/N 42.0S

LEL Drwg. \# 6V1765, $B=46.4$ [1:0hms] 5.11

$C=0.1$ [AFd], Atten. $=10.0$

HF Mod. $3421 \mathrm{~A}$

DOE 53252R

DOE 5S2215!Sy! 
Test Pracedure (Minimizing Rotational Error Effact)

Computer generated "prompts" instruct the operator to move the positioning fixture a total of 20 times for measuring and recording fluxlinkage. Changes in flux-1 inkage forresponding to 180 degree rotations of the finture about the $y$-axis of the coil-pair coordinate systeml are extracted from these data to determine three orthogonal components of magnetic moment.

For each measurement sequence, magnet identification and raw data - are saved along with the calibration constants needed to calculate magnetic mament fiom flux-linkage. At the end of the measurement sequence the program computes and displays information from which the operator may evaluate the data set. At any time the operator may restart or cancel the ineasurement sequence. At the end of a messuresnent sequence, the operator has the option of saving or not saving the frevious data, recalibrating, repeating measurements of the same magnet, measuring a different magnet, or terminating the data set (closing the file). 
A significant feature of our test-procedure/processing-algorithm is that it mininizes the effects of misalignment of the pos: ianing stage with respect to the Helmholtz coli-pair coordinate system (see figure 5). Outer surfaces of the positioning finture (a precision cube) mating with the horizontal and vertical surfaces of the positioning stage determine the orientation of the magnet under test. Misalignment of the positioning stage introduces 5ystematic angular errors which we have defined as roll, pitch, and $y a w$. We developed a measurement sequence and data reduction scheme that effectively cancels errors due to systematic piteh and yaw. (Because of the eylindrical symmetry of the coil-pair roll doesn't contribute slgnificantly to measurement error,) In order to illustrate the procedure we first, write Equation 2 that describes the flux-linkage of one of the specified fixture positions ffor other positions the equations the equations have the sane form). Next, we approximate tha cosine terms by 1.00, assumming roll, pitch, and yaw are small and reduce Eq, 2 to Eq. 3. Equations 4-a - 4-d represent the flux linkages due to the 4 orlentations of the positioning fixture for determining the $x$ ' component of magnetic moment.

$$
\begin{aligned}
& E(X)=\ln _{x^{*}} \cos (p i t c h) \cos (\text { yaw })+m_{y} \cos \left(\text { rollisin }(p i t e n)+m_{z} \cos (\operatorname{roll}) \sin (y a w)\right. \\
& E(3) \quad \sim+m_{x^{\prime}}+m_{r} \sin (p j t c h)+m_{z} \sin (y a w) \\
& E(2)=E(4) \sim-m_{x^{2}}+m_{y} \sin (p i t c h)-m_{z} \sin (y a w) \\
& E(3) \quad \sim+m_{x^{\prime}}+m_{r} \sin (p i t c h)+m_{z} \sin (y a w) \quad(4 b=3)
\end{aligned}
$$

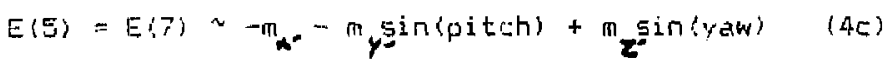

$$
\begin{aligned}
& E(b) \quad \sim+m_{x^{-}}-m_{y} \sin (p i t c h)-m \sin (y a w) \quad(4 d)
\end{aligned}
$$

Simultaneous solution of equations $4 a-4 d$ yields a value for $m_{x}$, while cancelling the other terms. Table VII indicates the orientations of the positioning finture during a measurement sequence, and suggests the way the data are combined to determine three companents of magnetic manent. 
Table VII MMSS 20 Measurement gequence Diagram

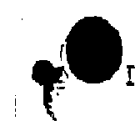

\begin{tabular}{|c|c|c|c|c|c|}
\hline $\begin{array}{l}\text { Vi ew } \\
\text { of } \\
\text { Cube }\end{array}$ & $x^{\prime}$ & $y^{\prime}$ & $z^{\prime}$ & $\begin{array}{l}\text { 1st } \\
\text { Diff }\end{array}$ & $\begin{array}{l}\text { Moment } \\
\text { (component) }\end{array}$ \\
\hline
\end{tabular}

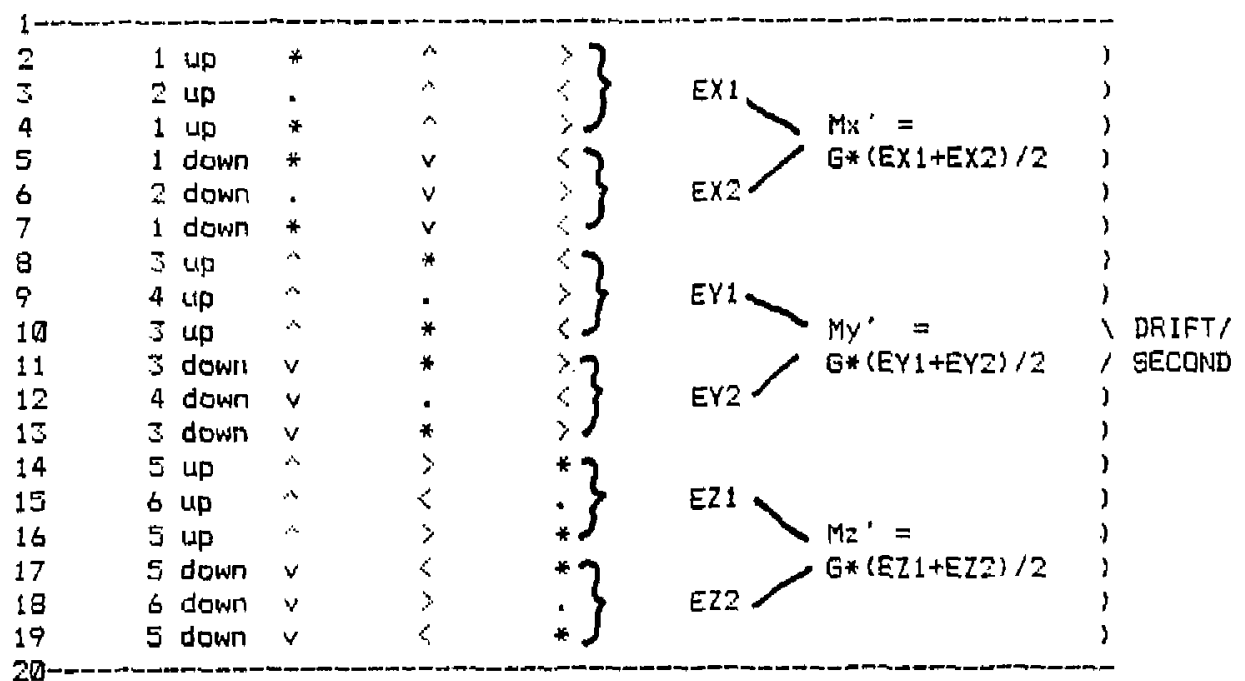

\section{DEFINITIONS OF TERMS \&SYMBOLS}

DEF INIT ION

\section{[UNITS]}

1

Sequence Number

$i$-th component of Magnetic Moment

$j^{\prime}=x^{\prime}, y^{\prime}$, or $z^{\prime}$

$E\left(M_{*}^{\prime}\right)+E\left(M_{2}^{\prime}\right) * \operatorname{SIN}(Y A W)$

$E\left(M X^{\prime}\right)-E\left(M z^{\prime}\right) *$ SIN (YAW)

$E\left(M y^{\prime}\right)+E\left(M z^{\prime}\right)$ * SIN (YAW)

$E\left(M Y^{\prime}\right)-E\left(M z^{\prime}\right)$ * SIN (YAW)

$E\left(M^{\prime}\right)+E\left(M_{Y}^{\prime}\right) * \operatorname{SIN}$ (YAW)

$E\left(M z^{\prime}\right)-E\left(M Y^{\prime}\right) *$ SIN (YAW)

Multiplier to covert pot-

ential to magnetic moment

[Dimensionless]

[Maiwell $\mathrm{Cm}$ ]

[V]

[U]

[V]

[V]

[V]

[V]

[Maxwel] cm/ Volt]

SYMEOL

(NOMINAL) DIRECTION

IN COIL-PAIF CDORDINATE SYSTEM

$$
\begin{aligned}
& -z \\
& +z \\
& +y \\
& -y \\
& +x \\
& -x
\end{aligned}
$$


Test Results

We meastred a total of 367 magnets for the ATA Beam Director.

Dne or more data sets far each of these magnets are filed in a Magnetic Measurements Encineering Data Book ond on Floppy Disks. Directories of these Floppies and a (measurement \& processing) log are includad in each bool:.

Figure b is a copy of information printed while messuring insgnet I.D. 301217. Figure 7 is a copy of one page of sarted data that includes magnet I.D. 301217. The sorting program uses "FT Sort" to search data files and to order records by selected criteria. The 367 magnets we mełsured were classified into 40 subsets like trie two shown in Figure 7 . Within each subset we sorted the magnets in order of decreasing magnitude of "Kry

Table VIII is an updated version of the Test Summary Table from ref8 erence 2. (At the time of publication of reference 2, dipoles had not been measured.)

Appendix A Sdistributed with the Nov. 85 status report; available on request) liste the scirted results of mensurements of Jba magnets. Appendix B (available on request) is a record of the blocte selerted for installation in 19 beam-line elements (dipoles and quadrupoles). Francis Younger of Brobeck Corporation seletted the magnet blocks for each element and produced the I ist represented oy Appendis $B$.

Afpendi: $c$ (not for general distribution) will document thre neesurements of 80 magnet blocks to be kept as spares. These blocks have not been measured (they were recieved at LEL on 85 November 27). 


\section{FICURE 6.}

\section{SAMPLE DATA SET}

DATH TAKEN DN 23-JUL-DE AT 15,29ISE

a

DATA ET AD2i07235.,ASC

MAGNET SI2E! 3

MAGNET OALENTATION: 1

MAENET MUMEERI 217

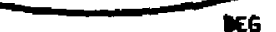

$$
\text { END }
$$

AVE DIF

\begin{tabular}{|c|c|c|c|}
\hline $\begin{array}{l}\text { EX [rolte] } \\
\text { EY (rolte] } \\
\text { E2 [voltes } \\
\text { Try [deo C] }\end{array}$ & $\begin{array}{r}-6.57655 \\
-0.01246 \\
6.0051 \\
72.74580\end{array}$ & $\begin{array}{r}-6.57786 \\
-.04372 \\
2.6465 \\
22.75709\end{array}$ & $\begin{array}{r}-6.57721 \\
-0.04259 \\
0.09548 \\
22.75180\end{array}$ \\
\hline
\end{tabular}

drift tav/second] $0.08 B 10$

nx MY Mr Mxy thtaxy

$-22539.71-149.69$, $19.46 \quad 225 \$ 1.18-179.43$

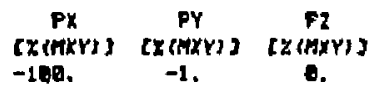

El apeed
Tiee
[5econds]
0.0
13.7
26.1
38.8
54.3
68.6
64.9
101.8
15.0
129.7

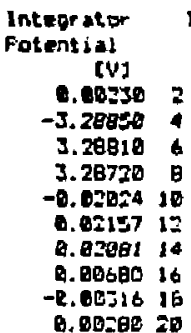

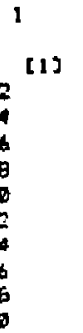

Elapsed
Time
[5econds]
8.5
19.6
32.7
46.6
62.9
77.6
94.4
88.4
21.6
38.5

Interatar Potential [V]

3.29998

3.28700

$-3.29020$

0.02957

0.02147

$-0.02255$

0.00228

0.60050

0.00445

D. 2448

\section{FACTOK: : \\ $0.5 \%$}

HELMHOLTZ RADIUE * D. $35:$

HELMHOLT2 TLFNS = \$77\%, ODC

ELFS SENSITIVITY = O.0E494?

AVERAGE CALJEFRTION = :0.727840 


\section{FIGURE 7.}

\section{SAMPLE SORTED SUBSET}

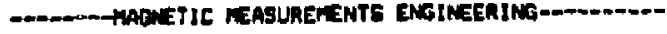

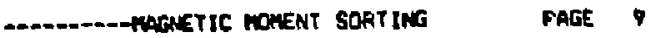

MAGNETS 4. IOS INEHES LONG WITH EASY AXIS DF 180.0 DEGREES MAGID HXY THAXY MZ DATE TIHE [Max-Cn] [Degreas] [Max-Cn]

\begin{tabular}{|c|c|c|c|c|c|}
\hline $\begin{array}{l}381217 \\
30115 \\
301137 \\
301169 \\
301401 \\
301155 \\
301177 \\
381195 \\
301389 \\
301129 \\
301161 \\
301185 \\
301145 \\
301145 \\
301201 \\
301153 \\
301137 \\
301193 \\
301129 \\
301185\end{array}$ & $\begin{array}{r}22533 . \\
22565 . \\
22649 . \\
22654 . \\
22695 . \\
23731 . \\
22893 . \\
22907 . \\
22933 . \\
22938 . \\
22969 . \\
23071 . \\
222580 . \\
223598 . \\
222653 . \\
222780 . \\
222915 . \\
222891 . \\
222992 . \\
225869 .\end{array}$ & $\begin{array}{r}-179.6 \\
176.1 \\
-176.9 \\
-179.5 \\
-177.4 \\
-173.6 \\
179.2 \\
-178.5 \\
-178.9 \\
-178.8 \\
178.4 \\
-178.4 \\
176.1 \\
176.5 \\
-177.4 \\
-173.6 \\
-176.9 \\
-178.8 \\
-178.6 \\
-178.5\end{array}$ & $\begin{array}{r}20 . \\
-147 . \\
254 . \\
179 . \\
-212 . \\
334 . \\
-271 . \\
416 . \\
-168 . \\
354 . \\
387 . \\
1101 . \\
-159 . \\
-159 . \\
-223 . \\
352 . \\
245 . \\
424 . \\
742 . \\
1896 .\end{array}$ & 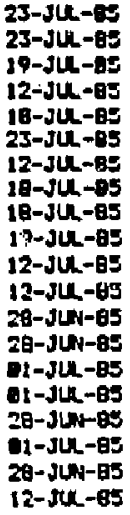 & 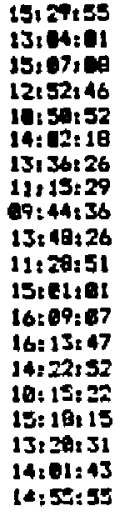 \\
\hline
\end{tabular}

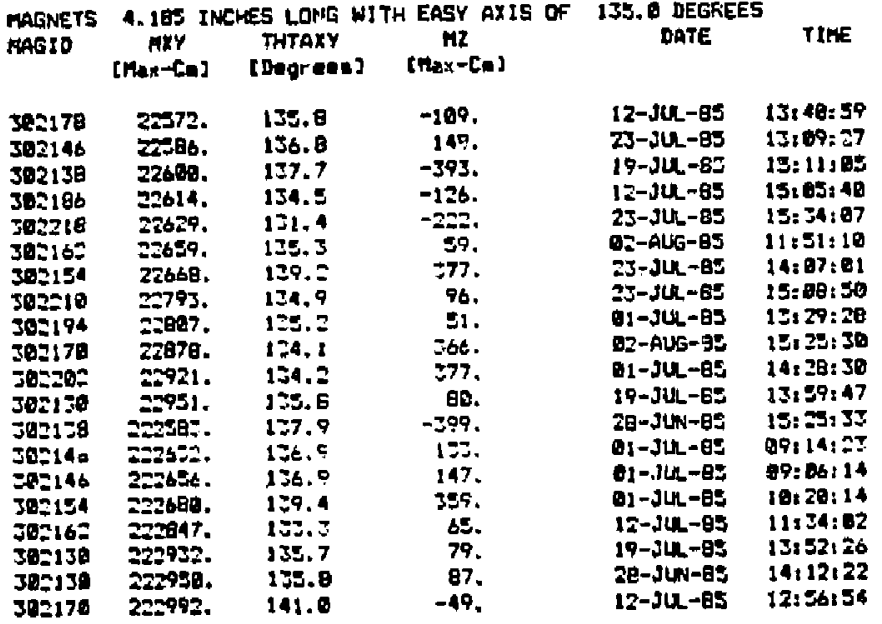


(Er OLIECL)

Dewo. No.

$951753-1$

$950175-2$

9501732

9501759

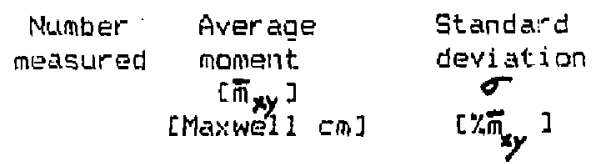

BQ

22626.

$47 * 11336$

96

22725

144

1551.5
1. 40

0.73

0.87

1.29
Voluns

[Y]

[5.]

6309

.304

3.4 .31

.609

3.438

7.721

3.509

Table VIII Suminary of Magnetic Monent Measurements

One magnet block (Drwg. No. 9501733-2, 5/N 126006) was danaged while being reworlied. Its magnetic imoment $m_{x y}(106006)=3602$ [Maxwell cm]s was rot ineluded in the average \& standard deviation data in Table VII 
Fieferences

1. Nelson, D.H., Green, M.I., Van Dyke, D.A., "Froposal for Measuring and Sorting Fermanent Magnet Blocks" LBL Engineering Note MT 546 , (LBID 995), February, 1985.

2. Nelson, D.H., Earale, F.J., Green, M.I., Van Dyke, D.A., "The Lawrence Erarkeley Laboratory Magnetic-manent Maasurement \& Sorting Systen, LEL Feport LEL 19292, July 1985, presented at the 7-th International Conference on Magnet Technology, Zurich Switzerland, September, 1.785 .

3. Macondray, F.w., "The 'Square Loop' Flus Standard: A Freacision Fulse Generatur". LEL Report UCFL 17a37, presented at the Second Intermatiomal Conferance an Magnet Technolog\%, D:Aford, England, July, 1967.

4. Magnetic Measurements Engineering Data Book Mo. S71-C, MMSs-2, May, 1985.

5. Magnetic Measurements Engineering Data Book No. 691-E,F,G, \&H, "DataSets ADO,AD1, f.: :, \& ADJ respectively, May through September, 1905.

Acknowl edgements

We thank Dr. Michael I. Green for his cantinuing suppurt throughout this praject - From specifying and structuring the CDAS through editing this Engineering Note. We thank Paul Earale for programming support, especially for lis effort in programming with RTSORT. We thank Lr. Edward F'. Lee for supporting our request to develop the MMSS.

Distribution

J. Alonso

A. Arthur

F. Barale

G. Cruz (LLINL)

C. Dols

W. Euliel, (Brobect)

$B_{n}$ Feinberg

M. I. Green

K. Halbach

E. Hartwig

E. Hoyer

C. Huddlesten (NSWC White Oak Laboratory Silver Springs, MDl 20910)

B. KuIke (LLNL)

E. LeE

E. Nolting (NEWC White Oak: Laboratory Silver Springs, MDK 20910)

D. Shuman

J. Tanabe

D. Van Dyke

F. Younger (Erobeck)

Magnetic Measurements Engineering (4)

Electronics Enginegring Dept. (Original + 2) Technical Information Dept. (I) Authors 


\title{
$4000-300 / 301$
}

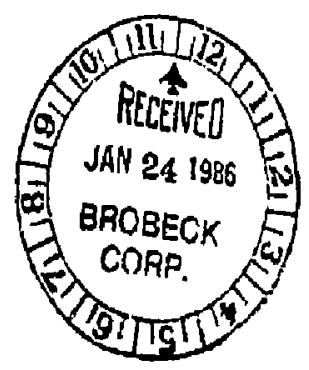

MAGNETIC MEASTREMENTS

$$
\text { O I T H E }
$$

A TA BEAM DIRECTOR

PERMANENT MAGNET

ACBROMAT Q TADRUPOLES

\author{
AUTHORS: Hichael I Grean \\ Paul J Barale \\ David A Van Dyke \\ Magnetic Measurements Engineering Group \\ Lawrence Berkeley Laboratory \\ Dniversity of California \\ Berkeley, California 94720
}

MT NOTE $\sharp 357$

20 December, 1985

*This work was supported by the Director, Office of Energy Research, Offlce of High Energy and Nuclear Physics, High Energy Physics Division, U.S. Dept. of Energy, under Contract No. DE-ACO3-76SFפOB98. 
PAGE 1

\section{TABLE OE CONTENT S}

1.0 INTRODUCTION .................. 2

2,0 HARDHARE ..................... 2 TABLE 1.

FIGURE 1.

3.0 SOFTHARE ................... 6

4.6 SETOP AND ALIGNMENT. . . . . . . . . . . . . .6

4.1 CONCENTRICITY ALIGNMENT . . . . . . . . . 6

4.2 ZERO-INDEX ALIGMNENT. . . . . . . . . . . . 6 FIGURE 2.

5. QUADRDPOLE FIELD STRENGTH. . . . . . . . . . 7 TABLE 2.

6. GUADROPOLE AZIMUTHAL ORIENTATIONS, , , . , , . 8 FIGURE 3.

TABLE 3.

7.6 EARMONICS . . . . . . . . . . . . . . . . . . FIGURTS 4. TO 11.

8.0 ACKNOHLEDGEMENTS. . . . . . . . . . . . . . . . .16 APPENDIX A LISTRIBUTION 


\section{INTRODOCTION}

Ed Lee requested that the LBC Macnetic Neasurements Engineering (MNE) Group measure the characteristics of the ATA Beam Director Achromat Focusing and Defocusing Quadrupolo Hagnets (Brobeck Company Drawing Numbers 95D1732 95D1733). Each magnet is fabricated frow sixteen oriented-grain-ferrite permenent-magnet-blocks, arranged in a clrcular array to form a permanent magnet quadrupole. There are eight such arrays with length of $10.63 \mathrm{~cm}$ and two arrays with a length of $5.315 \mathrm{~cm}$. Of the eight "long" quadrupoles, four are designated "focussing" quadrupoles ( $1 F, 3 F, 4 F \& 5 F$ ) and four are "defocussing" quadrupoles (2D, 6D, 7D, BD). The beam tube aperature radius of the permanent magnet quadrupoles is $1.42 \mathrm{~cm}$.

We have measured the strencth, and azimuthal orientation of the quadrupoles. Also measured and analyzed are the error hamnonics and the dipole content of the quadrupole fleld. The original data is stored in MNE LOB Book $\$ 768 \mathrm{~A}$ and on floppy disks HG305, MME315.

\section{D HARDWARE}

The hardware used to measure the ATA Beam Director Permanent Magnet Quadrupoles was the MME suall quadrupole system.

The search coll drive mechanism used was one of the Magnetic easurements Group standard systems and is dosumented on LBL drawing 17M3273. his drive operated one of the Magnetic Measurements Group's standard search coils, known as the BILAC coil $\#$. The parameters of this search coil are documented in the subroutine SCDSC3. Our standard data acquisition hardware (Search Coil Switching Module, $\nabla / f$ Module and NIM 3 Channel Op/Down Counter), and monitoring and control units were used. Detuils of the hardware used and a block diagram detailing the system interconnections are given in Table 1 and Figure 1 respectively. 


\section{PAGE 3}

TABLE 1: ATA PERMANENT MAGNET QOADRUPOLE HARMONIC ANALYGIS HARDHARE

MAGNET

COMPUTER

MEMORY

CLOCK CALENDAR

LINE PRINTER

GRAPHICS PRINTER

CRT CONTROL TERMINAL

FLOPPY DISC DRIVE

HARD DISR DRIVE

WINCHESTER CONTROLLER

INCHESTER DRIVE \#1

WINCHESTER DRIVE \#2

PLOTTER

BARDCOPY

*GPIB INTERFACE

*HFX.

*DVM1

CAMAC CRATE \& PS

CAMAC CONTROLLER

INPUT/OUTPUT REGISTER

CAANGE-OF-STATE MODULE

HARY INPUT MODULE
ATA BEAM DIRECTOR - PERMLNENT MATNET QUADRUPOLE BROBECK DWGS 95D1732 AND 95D17433

DEC LSI $11 / 73$, S/N SG14285641

CAMINTONN 1 MBYTE RAM S/N 2155

DIGITAI, PATEKAYS, TCU-50DKR, S/N 16574

DEC LA 129, DECHRITER III, DOE 519478

EPSON FX-185, DOE 534943

GRAPHON G0160, DOE 534949

DATA SYSTEMS DSD 440, DOE 519465

DEC RLD2, DOE 522851

MTI MLV11K-2, S/N $1687 \mathrm{E}$

RODIME MODEL RD 204, S/N 2493571

RODIME MODEL RO 204, S/N

TEKTRONIX 4051, DOE 504556

TEKTRONIX 4631, DOE 504505

NATIONAL INSTRONENTS GPIB118-1, S/N 145

HEWLETT PACKARD MODEL 3495A SCANNER, OPTIONS OO5, 100, DOE 517528

HENLETT PACKARD MODEL 3455A DIGITAL YOLTMETER, DOE 517459

STANDARD ENGINEERING, MODEL 3472 CRATE \& PS DOE 512946, PS S/N 2683

STANDARD ENGINEERTNG, CCLSI-11, DOE 524892

JOERGER MODEL IR-1 IN/OUT REGISTER, DOE 512965

BI RA 3326 DIGITAL INPUT MODULE S/N 663

STANDARD ENGINEERING PR604 DOAL I/P REGISTER S/N 0393 
PAGE 4

NARY OUTPUT MODULE STANDARD ENGINEERING PR612 DUAL O/P REGISTER S/N 0926

RELAY SWITCH MODULE RINETIC SYSTEMS 3075-E1A RELAY OUTPUT REGISTER S/N 83

SEARCH COIL DRIVE MOTOR

LBL DWG $17 \mathrm{MB273}$

BODINE NO. 766HA5014, TYPE KYC-22T5, 2 RPM

ROT. OPT. ENC. DISC INSTR. ROTASWITCH MODEL 811-256-IBLS-TTL ADAPTER BOX

NIM BIN

ORTEC 401B

- NIM BIN PS

ORTEC MODEL 1620, S/N 2340

NIM 3 CHAN OP/DONN

COUNTER

LBL DWG 16V1323, S/N 1

$\checkmark / F$ CONVERTER

LBL DHG 16V120, S/N 1, DOE 521827

SCSM

SEARGH COIL SWITCHING MODULE, HITH PREAMP LBL DFG 16V121, S/N 1

CABLE 1

LBL DWG 16Y124-1

- Garch coll arRay \& SUBROUTINE SCDSC3 BILAC $\$ 5$

SOFTWARE

QD2.SAP 94 NOV 85 -CONTROL PROGRAH

- *INDICATED DEVICES AVAILABLE BUT NOT USED NITH THIS SYSTEM 


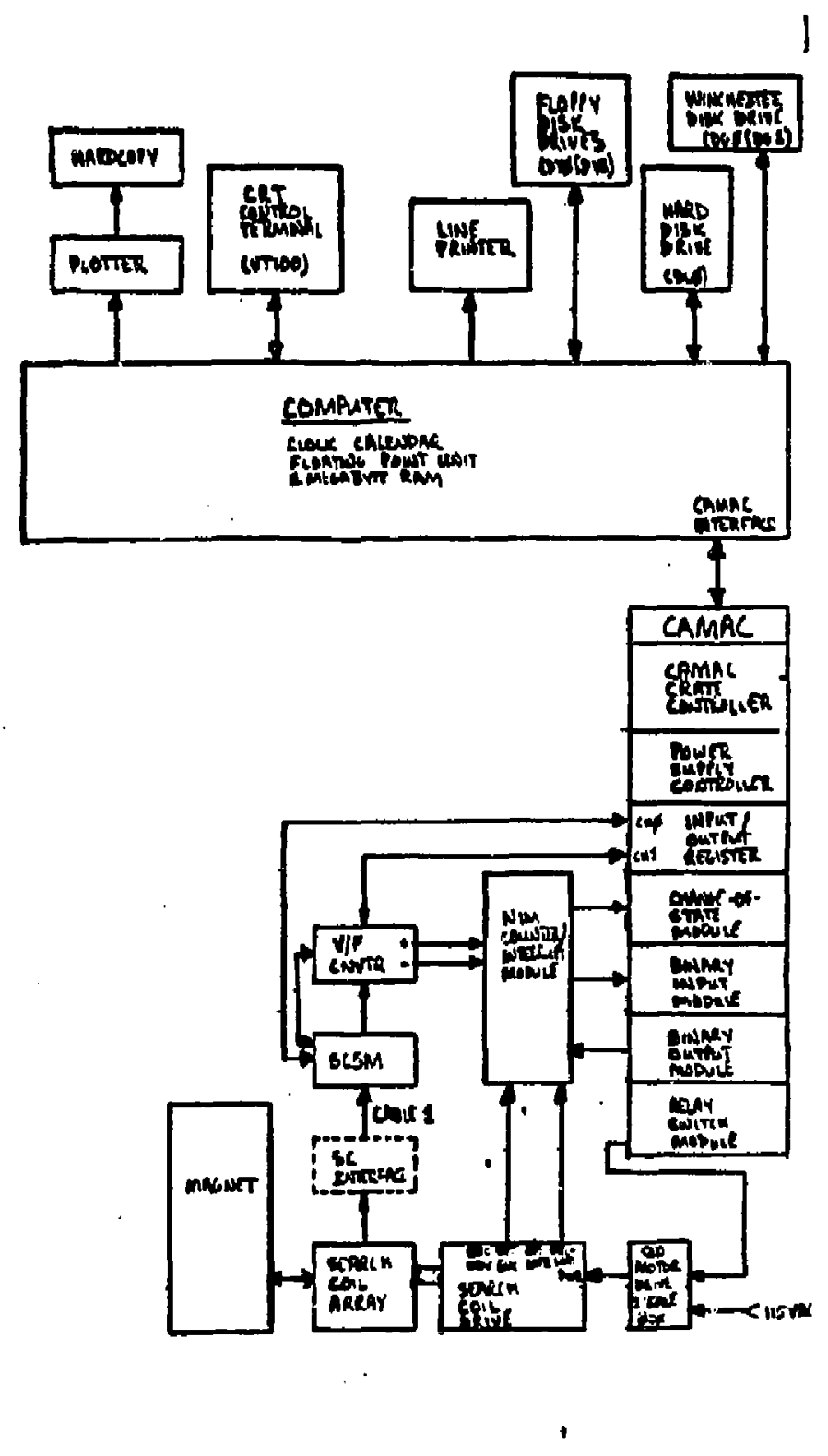


MAGNETIC MEASUREMENTS

$$
\text { OE T } \mathrm{HE}
$$

ATA BEAM DIRECTOR

PERMANENT UAGNET

ACBROMAT DIPOLES

\author{
AUTHORS: Michael I Green \\ Paul J Barale \\ David A Van Dyke \\ Magnetic Measurements Engfneering Group \\ Lawrence Berkeley Laboratory \\ Oniversity of California \\ Berkeley, California 94720
}

UT NOTE $\$ 358$

20 December, 1985

*This work was supported by the Director, Office of Energy Research, Office of High Energy and Nuclear Physics, High Energy Physics Division, O.S. Dept. of Energy, under Contract №. DE-ACD3-76SEQB998. 
PAGE 1

$$
\text { TA B E OE C ONTENTS }
$$

1.0 INTRODUCTION . . . . . . . , . , . . 2

2.D BARDHARE ................... 2

TABLE 1.

FIGURE 1.

3.D SOFTHARE ................... 6

4.6 SETUP AND ALIGNMET. . . . . . . . . . . . . . . . 6

4.1 CONCENTRICITY ALIGMENT . . . . . . . . 6

4.2 ZERO-INDEX ALIGNMENT. . . . . . . . .6

5.6 DIPOLE FIELD STRENGTH. . . . . . . . . . . . 7 TABLE 2.

6.も DIPOLE AZIMUTHAL ORIENTATIONS. . . . . . . . . . 8 FIGURE 2.

7.6 HARMONICS . . . . . . . . . . . . . . . . . . . .

FIGURES 3. TO 10.

B. ACENONLEDGEMENTS. . . . . . . . . . . . .10 APPENDIX A DISTRIBUTIOU 


\subsection{INTRODUCTION}

Fd Lee requested that the LBL Magnetic Measurements Encineering (MIE) Group measure the characteristics of the ATA Beam Director Achromat Dipole Magnets (Brobeck Company Drawing Number 95D1734). Each magnet is fabricated from sixteen oriented-crain-ferrite permanent-magnet-blocks, arranged in a circular array to form a parmanent magnet dipole. There are nine such arrays with a length of 9.00 on. The bean tube aperature radius of the permanent magnet dipoles is $1.42 \mathrm{~cm}$.

We have measured the strength and azimuthal orlentation of the dipoles. Also measured and analyzed are the error harmonjcs and the dipole angular deviation. The original data is stored in ME Log Book \#7BBB and on floppy disks MG365. HIE315.

\subsection{BARDAARE}

The mechanical hardware used to measure the ATA Beam Director Permanent Magnet Dipoles was the ME small quadrupole system adapted for use in measuring the dipoles. The search coll drive mechanism used was one of the Magnetic Measurements Group standard systems and is documented on LBL drawing 17M3273. This drive operated a new search coil array, known as the "30mm.OD Dipole 2-pair search coil". This search coil was designed and fabricated specifically for these measurements. It and - 5 documented on LBL drawing 167133, and it operating parameters are ammarized in the subroutine SCDSC7. Our standard data acquisition hardware (Search Coll Switching Module, $\nabla / f$ Module and NIM 3 Channel Op/Down Counter), and monitoring and control units were used. Detalls of the hardware used and a block diagram detailing the system interconnections are given in Table 1 and Figure 1 respectively. 
TABLE 1: ATA PERMANENT MAGNET DIPOLE HARMONIC ANALYSIS HARDWARE

MAGNET

COMPUTER

MEYORY

CLOCK CALENDAR

LINE PRINTER

GRAPHICS PRINTER

CRT CONTROL TERMINAI,

FLOPPY DISC DRIVE

BARD DISK DRIVE

WINCEESTER CONTROLLER

INCHESTER DRIVE \#1

WINCEESTER DRIVE *2

PLOTTER

EARDCOPY

GPIB INTERFACE

MPX

DRM1

CAMAC CRATE \& PS

CAMAC CONTROLLER

INPUT/OUTPUT REGISTER

CBANGE-OF-STATE MODOLE TINARY INPUT MODULE
ATA BEAM DIRECTOR - PERMANENT MAGNET DIPOLES BROBECK DHG 95D1734

DEC LSI $11 / 73$, S/N S614285641

CAMINTONN 1 MBYTE RAY S/N 2155

DIGITAL PATHKAYS, TCU-50DYR, S/N 16574

DEC LA 120, DECWRITER III, DOE 519478

EPSON TX-185, DOE 534943

GRAPHON G0160, DOE 534949

DATA SYSTEMS DSD 440, DOE 519465

DEC RLø2, DOE 522851

MTI MLP11H-2, S/N 16875

RODIKE MODEL R 264, S/N 2463571

RODIME MODEL RO 204, S/N

TERTRONIX 4851， DOE 504556

TERTRONIX 4631， DOE 504565

NATIONAL INSTRUHENTS GPIB11V-1, S/N 145

HEWLET'T PACKARD MODEL 3495A SCANMER, OPTIONS DO5, 109, DOE 517528

HEWLETT PACKARD MODEL 3455A DIGITAL VOLTMETER, DOE 517459

STANDARD ENGINEERING, MODEL 3472 CRATE \& PS DOE 512946, PS SN 2683

STANDARD ENGINEERING, CCLSI-11, DOE 524892

JOERGER MODEL IR-1 IN/OUT REGISTER, DOE 512965

BI RA 3326 DIGITAL INPUT MODULE S/N 663

STANDARD ENGINEIRING PR694 DUAL I/P REGISTER S/N $\varnothing 393$ 
PAGE 1

INARY OOTPUT KODOLT

RELAY SWITCH HODULE

SEARCH COIL DRIVE HOTOR

ROT. OPT. INC. ADAPTER BOX

NIM BIN

NIM BIN PS

NIM 3 CHAN UP/DOWN COUNTER

V/F CONVERTER

$\operatorname{SCSM}$
STANDARD ENGINEERING FRG12 DUAL O/P REGISTER S/N B325

KINETIC SYSTEHS 3E75-EIA RELAY OUTPUT REGISTER $\mathrm{S} / \mathrm{N}$ B3

LBL DHG 17M3273

BODINE NO. 766HA5014, TYPE KYC-22T5, 2 RPM

DISC INSTR. ROTASWTTCH MODEL 811-256-IBLS-TTL

ORTEC 461B

ORTEC MODEL 4629, S/N 2340

LBL DNG 16V1323, S/N 1

LBL DNG 16V129, S/N 1, DOE 521827

SEARCH COIL SHITCHING MODULE, WITH PREAMP LBL DWG 16V121, S/N 1

SEARCH COIL ARRAY \& SUBROUTINE

SCDSC7

3Gmo OD DIPOLE 2-PAIR SEARCB COIL;

LBL DHG $16 V 133$

DP5.SAV 28 SOY 85

- CONTROL PROGRAM 
PAGE 6

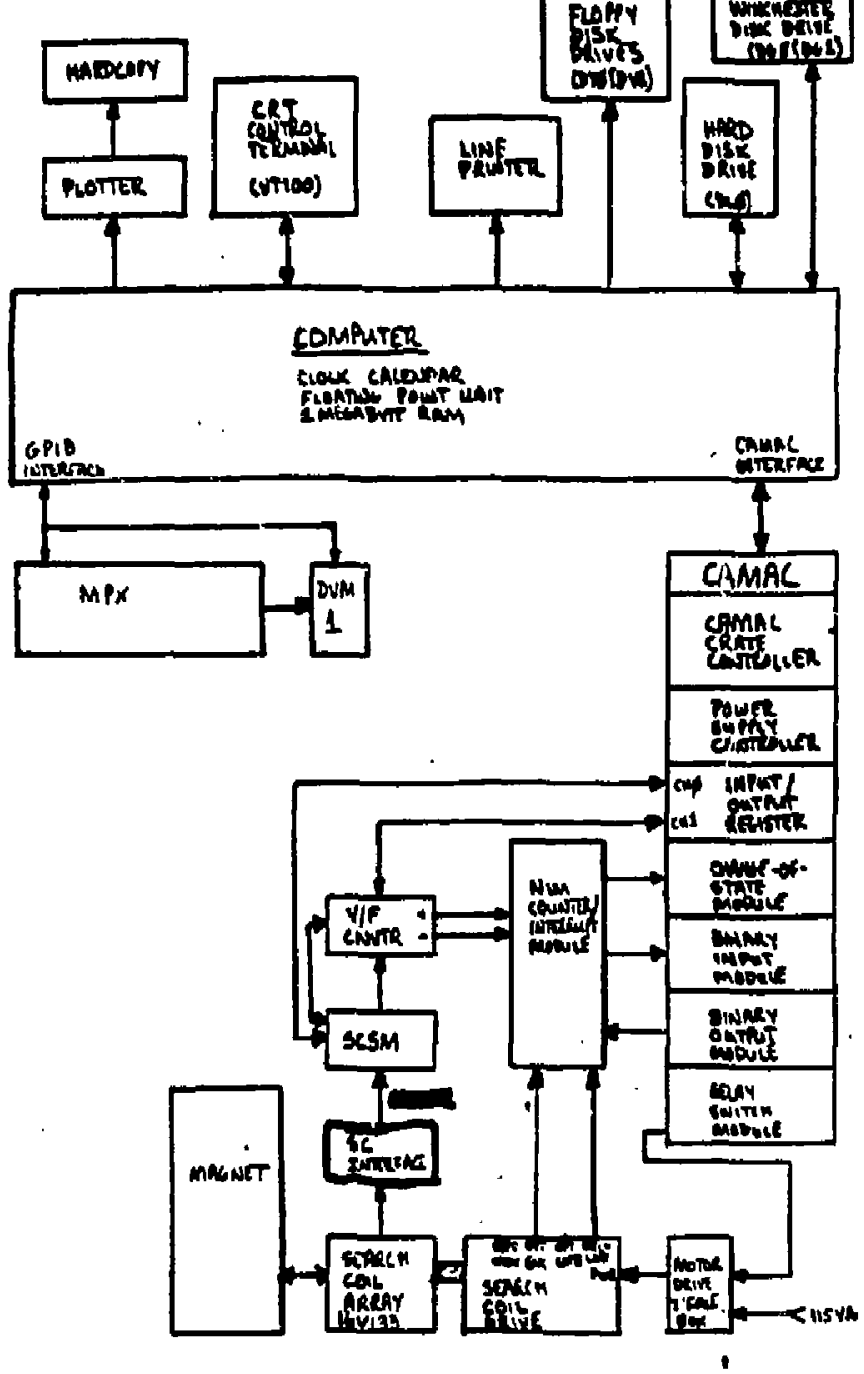

FIGURE 1.

BARMONIC ANALYSIS MEASUREMENT HARDWARE BLOCK DIAGRAM 
UAG AETIC MEASORINENT

$$
\text { OF } \mathrm{TB}
$$

ATA BEAL DIREOTOR

TERHIER DIPOEE HAGNE *

AOTBORS: Paul J Barale Hichael I Groen

David A Pan Dyke

Bagnetic Heasuremonts Engineering Group Lawrence Berkeley Laboratory

Dolversity of Californta

Berkeley, Californta 94720

*This work was supported by the Director, Office of Inerey Research, Office of Bigh Eriergy and Nuclear Physles, High Enercy Phyrles Division, 0.6. Dept. of Enercy, under Contract No. DE-AC93-76ST68698. 
TABLI OT COMTIATS

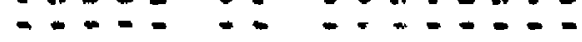

1.0 INTRODNCTION . . . . . . . . . . . 2

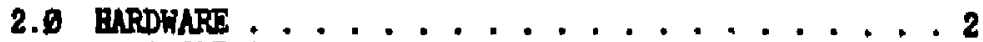
TABLS 1.

DIGURE 1.

3.0 GOETTARE . . . . . . . . . . . 6

4.0 getur and allghaBnt. . . . . . . . . . . 6

4.1 axial alighant . . . . . . . . . . . 6

4.2 A2IKOTHAL ALIGALRTT . . . . . . . . . 6

4.3 CONCENTRICITY ALIGNAENT . . . . . . . 7

5.0 SRARCB COIL ROTATIOA SPRED TESTS . . . . . . . 7 TABLE 2.

6.0 IMGNET COIL RESTSTANCE / TEMPIRATURES. . . . . . 6 TABLB 3.

1.0 DIPOLE FIRLD STRWHGTH. . . . . . . . . . . . 9 TABLB 4.

8.0 DIPOLE AZIYTTHAL ORIENTATIOHS. . . . . . . . . . . FIGURB 2.

8.6 MTAL GBNTIR . . . . . . . . . . 13 EIGURE 3.

10.9 BLRHONICS. . . . . . . . . . . . . . . . . . IIGURES 4. TO 11.

11.0 ACRAOHLEDGMENTE . . . . . . . . . . . . 16 DISTRIBOTION . . . . . . . . . . . . 25

APFEHDICES

A. DIRECTORY OF SOFTHARE FILES

B. SOMMARI OF FIELD AKD POKER DATA

C. GOMMRY OF DATA OSED TO CALCOLATE AZIMOTEAL ORIENTATIONS

D. STREARY OF DATA USED to CALCOLATE AXIAL CENTERS 


\subsection{IMTRODDCTION}

Id Lee requeated that the IBC Mapotile Hensurenents Inglneerib: Croup neasure the operatios characteristies of the ATA Been Diroctor Vernior Steerlas Hacnot enpufactured by the Brobeck Company. Thts tronless varnet consists of two parpondicular dipolo colle approxisately 16 on lonc, wound on $2^{n}$ I.D. bore tube.

He have measured the transfer function, harmonics, esiathal orlentation, effective exial position, and coli power requirenonts. The oridinal data 18 stored in Ma LCH Book 694 and on floppy disks Yo272, His282.

\subsection{BARDAARE}

The hardware secup used to neasure the ATh Beam Director Fernier macnet was a variation of a typical setup used for weasurement of $c$ superconducting dipole. The macmet's inger and outer colls were powered alternately using ar adjustable 40 volt, 50 anp power supply. The search co11 drive mechan1sm used was borroked fron the Supercon Group, and drove an obsolete Supercon Group search coll array. Our standard data acquisition hardware (Search Coll Ewitching Hodule, $\nabla / f$ Hodule and NIM 3 Channel Op/Down Counter), and nonftoring and control units were used. Our universal water napifold was used to provide low conductivity water for magnet cooling. Detalls of the hardware used and a block diagram detalling the system interconnectlons are given in Table 1 and Figure I respectively. 
MAONET

COMPOTRR

MEMORY

CLOCR CALBNDDAR

LINE PRINTER

GRT CONTROL TERMINAL

FLOPPY DISC DRIVE

BARD DISR DRIVE

WIHCEESTER COMTROLLER

WINCHESTER DRIFE

PLOTTER

BARDCOPY

GPIB INTERFACB

MPX

DWM1

CAMAC CRATE \& PS

CAMAC CONTROLLER

INPOT/OOTPOT RBGISTER CBANGE-OF- STATE HODOLE BINARY INPOT MODULE BINARY OITPOT YODOLE RELAY BWITCB BODOLE
ATA BRAY DIRTCTOR - 2 DEGRAR VRRNIER DIPOLE DIC LSI 11/73, 8/N 8614285641 GAMILTONA 1 KBYTE RAY 6/N 2165 DIOITAL PATETAYS, ICD-6EDYA, 8/N 16574 DEC LA 120, DECWRITER III, DOR 519478 DEC VT160, DOR 626336

DATA SYSTIMS DSD 440, DOE 518465

DEC RLO2, DOE 622851

MTI RLP11M-2, S/N 16875

RODIKE HODEL RO 204, S/N 2493571

TIKTRCNIX 4051, DOR 504556

TEKTRONIX 4631，DOR 504565

DIATIONAL INSTROMENTS GPIB117-1, SN 145

EETILETT PACKARE HODEL 9495 SCAMNER, OPTIONS 605, 1618, DOR 517628

IEWLETT PACTARI) MODEL 3455A DIGITAL POLTRETER, DOR 517459

STANDARD HNGINERRING, HODEL 3472 CRATE \& PS DOE 512846, PS S/N 2683

STANDARD SNGIHIDERING, CCLSI-11, DOE $\$ 24892$

JORRGRR MODEL IR-1 IN/OOT REGISTER, DOR 512965

BI RA 3326 DIGITAL INPOT HODULE S/A 963

STANDARD ENGINEERING PR604 DOAL I/F REGISTER SA 6393

STAKDARD BNGINKERING PR612 DUAL O/P REGISTER $8 / \mathrm{N}$ 6326

RINETIC EYSTEMS 3075-E1A RELAY OLTPUT REGISTER $8 / \mathrm{N} 63$

LBL DWG 16132 (GMLLL PORTABTE BOX) 
PAOT 4

- MOTOR DRIVE ILSCTROAICS

GOTOR IITTRAACL

WDTOR CONTROLLTR

WOTOR

SEARCE COIL DRIVE ROT.OPT. ENC.

NIM BIN

NIM BIN PS

NIM I CEAN OP/DOWN COUNTER

V/T CONVERTER

SCSH

GEARCB COIL ARRAY

DIPOLE PS

CONTROL POLTAGE PS

PS SHUNT

IMNER COIL SHOWT

OOTBR COIL GEONT

DVH2

WATER MANIFOLD
IILD MAPPING YOTOR RTVRRIIA BOX LBL DHG 134567

MINARIR HODIL TRgoro (old out 84 oct 25) (now in 64 oct 26 1490 labelod)

TLICTRO CRATT CORP. WOTORMTIC MOTOR GENERATOR, PART 10. 0650 972, 6N \& 63880 (out B4 oct 25) $B / N$ B 65062 (in $B 4$ oct 251400 hours)

LBL DNG P7P?P???

DISC IHSTR. ROTASHITCE HODEL 811-256-IBLS-TTL

ORTEC 461B

ORTEC HODEL 4620, S/N 2340

LBL DHG 16V1323, S/N 1

LEL DHG 167129, S/N 1, DOE E21827

SBARCG COIL GWITCHING HODOLE, WITH PRRAMP LBL DNG 167121, B/N 1

LBL DHG 19H8144A-G (1.2" OD ALUMINUA)

BARRISON HODEL 6269A, 40 FOLTS, 50 AMPS SN 118; ARC $\because 104587$

LAIBBDA HODEL LP-526-FH

REGOLATED P.S.; OV - 10V - 5AMPS, S/N B14140

LETODE \& HORTHROP, CAT. NO. 4361, B/N 792989

0.01 OHLS, 160 AMP

BMPRO HA-25-50

25 AMP, 58 LT

0.092000 OHIS $5 \mathrm{~A} ; 0.002001$ OAMS $20 \mathrm{~A}$

TMPRO BA-25-50

25 NIPS, 50 MT

0.902001 OHMS - 6A; 0.602001 OHHS $20 \mathrm{~A}$

(USBD TO YONITOR CONTROL POLTAG' P.6.)

FLOKE 77 S/N 35676710 - BAND HELD VOH

LBC DHG 16V131 


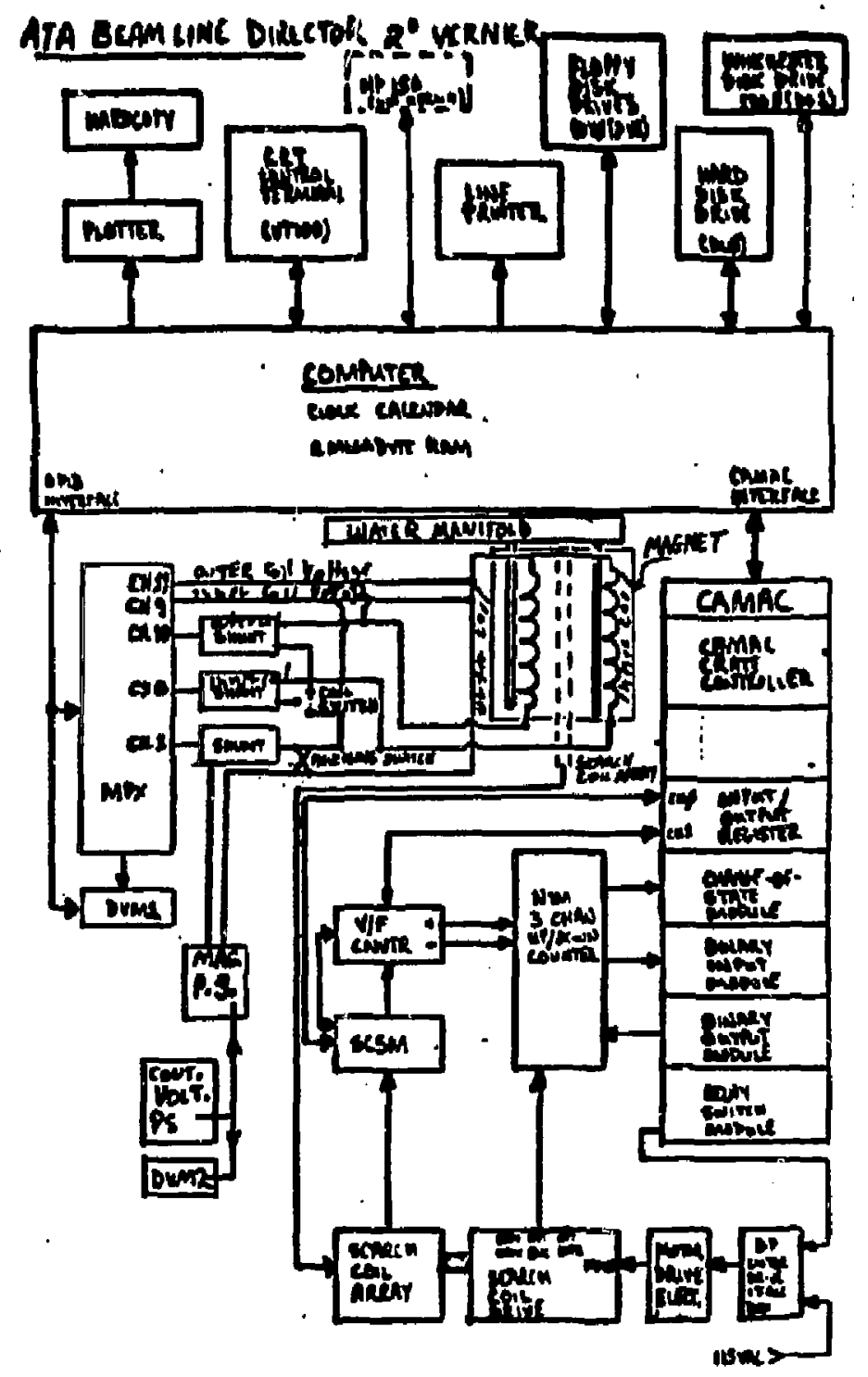


APPENDIX G. COMCEPTUAL DESIGN OF AN ELECTRON BEAM DEFLECTOR 
CONCEPTUAL DESIGN OF

AN ELECTRON BEAM DEFLECTOR

For

LAWRENCE BERKELLEY LABORATORY

1 Cyclotron Road

Berkeley, Callfornia 94720

PRINCLPAL INVESTIGATOR

Francis C. Younger

By

BROBECR CORPORATION

1235 lenth Street

Berkeley, Callfornia 94710

(415) 524-8664

FINAL REPORT NO, 4500-132-16-R1 
APPROVALS

Issue $\quad$ Contents

Original $v+75$ pages

Prepared by:

F. C. Younger

A. M. Weston

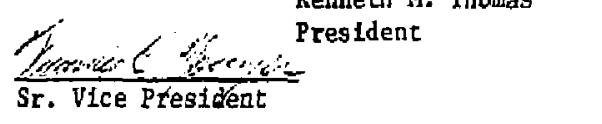

Checked by

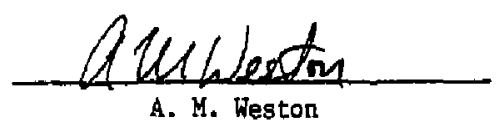

A. M. Weston

1

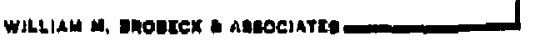


TABLE OF CONTENTS

PAGE

ABSTRACT

1.0 INRRODUCTION 3

2.0 CONCEPTUAL DESIGN 5

2.1 Magnetfc Lattice 9

2.2 Vacuum Rotary Joints 25

2.3 Vacuum-to-Air Transftion 28

2.4 Vernier Steer1ng System 33

2.5 Mechanical Mounts and Slewing System 36

2.6 Radiation Effects 65

2.7 Stze, Weight and Cost Esttmate 68

3.0 RECOMMENDED RESEARCH AND DEVELOPMENT 70

$\begin{array}{lll}4.0 & \text { CONCLUSIONS } & 73\end{array}$

5.0 APPENDIX 


\section{LIST OF FIGURES}

\section{$\underline{\text { PAGE }}$}

2.0-1 Conceptual Des1gn of Electron deam Director 6

2.0-2 Beam Direction in Spherical Coordinates $\theta$ and 8

Shows Dependence on Rotation $\alpha_{1}$ and $\alpha_{2}$

2.1-1 Magnetic Iattice Showing the Arrangement of

Quadrupoles and Bending Magnets (dIpoles)

2.1-2 Rare-Earth-Cobalt Permanent Magnet With. BxH = 30 wGoe 15

2.1-3 Rare-Earth-Cobalt Permanent Magnet with Bxil $=26$ xGOe 16

2.1-4 Rare-Earth-Cobalt Permanent Magnet with $\mathrm{BxH}=18 \mathrm{KGOe} 17$

2.1-5 Oriented Ferrits Permanent Magnet with $\mathrm{BxH}=3.3 \mathrm{MGOe} 18$

2.1-6 Permanent Magnet Dipole and Quadrupoles 19

16 Trapezoldal Pleces Magnetized in Direction of Arrows

2.1-7 Dipole and Quadrupole Magnet Weights 21

2.1-8 Section of Bending and Focussing System 24

2.2-1 Upper Vacum Rotary Jo1nt 27

2.3-1 Vacuum-to-Atr Transition with High Speed 32 Rotating Disk

2.4-1 Vernler Steer1ng Magnet $\quad 34$

2.4-2 Steering Magnet Coll Cross Section 35

2.5-1 Gyrodynamic Load Calculation Schematic Dfagram 42

2.5-2 6 Degrees of Freedom, Arls 1 System Schematic 48

2.5-3 6 Degrees of Freedom, Axis 1 System Equation Matrix 49

2.5-4 Structural Cross Sections for Estimation of Beamlike 51 Flexibilities 


\section{LIST OP FIGURES}

PAGE

2.5-5 6 Degrees of Freedom, Preliminary Axis 2 System Model 53

2.5-6 6 Degrees of Freedom, Preliminary Axis 2 System Model 54

2.5-7 Gee Load Versus Time at Axis 2 Support Bearing 56

2.5-8 Dynamic Multipliers for Triangular Pulse Versus 57 Pulse Time to Natural Period Ratio

2.5-9 Slewing System - Total System Weight Versus 60 Transition Section Dynamic Translation

2.5-10 Slewing System - Axis 1 Drive Borsepower Versus 61 Transition Section Dynamic Translation

2.5-11 Slewing System - Transition Section Dynatalc Rotation 62 Versus Transition Section Dynemic Translation

2.6-1 Beam Loss Due to Radiation and Collisions for Be and $C$ 67 Foils and for Air 
LIST OF TABLES

PAGE

2,1-1 Summary of Lattices Calculated 23

2.3-1 Calculated Temperature Rise for Varlous Elements 29

2.5-1 Weight Summary for Axis 2 System 39

2.5-2 We1ght Sumary for Axis 1 Sygtem 40

2.5-3 Absolute Acceleration of Point P in Whirling 43 xyz Coordinate System

2.5-4 Whirl and SpIn Rinematics 44

2.5-5 Axis 2 Gyrodynadic Calculaition Station Input 45

2.5-6 AxIB 1 Gyrodynamic Calculation Station Input 47

2.5-7 Steel Structure Matrix Coefficients and Load 52 Vectors

2.5-8 Aluminum Structure Matrix Coefficlents and Load 52 Vectors

2.5-9 Tabulation of Dynamic Kultiplier Data 58

2.5-10 Summary of Estimated Peak Mechanical Responses 63

2.7-1 Sumary of Size, Weight \& Cost 69 


\section{ABSTRACT}

A design of an electron beam director has been conceptualized and analyzed. Its performance objecti": is an ability to rapidly and accurately direct a high-intensity pulsed-besm of high-energy electrons to any desired $t^{n}$ :get spct within a 60 degree holf-angle cone. The beam director has a number of elements which can be separately analyzed, but which must all be integrated to give a sultable overall design concept. The separate elements of the design study are the following:

- Nagnet Lattice

- Vacuum Rotary Joints

- Vacuru-to-Air Transicion

- Verafer Steering System

- Mechantcal Kount and Slewing System

- Radiation Effects

- Size, Weight and Cost Estimate

The magnet lattice design has been reviewed for acceptabie beam optics, achromaticity, mechanical integrity and quality matertals avallability. A review of vacuum requir ments was made with due consideration to rotary joints In the tube, beam loss to vacum wall causing outgassing, and feasibility of a Iotatiog foil mechanism at the exit end of the beam director.

Analysis of a typical mount and associated vibration was made using slewing rates fast enough to respond to firing multiple prises anywhere in a $60^{\circ}$ cone within one second. Electronic fine tuning and wechantcal sensors have been considered for accurately controlling the bean director within the short time period.

Radiation has been studied to the extent it affects the vacuum and mechanical operation.

An estimate of size, weight, and cost has been made for the conceptual design.

* This effort has been coordinated with Dr. Edward P. Lee, Lawrence Berkeley Laboratory, and we acknowledge his valuable advice and guidance. 
Since this is a conceptual design, unusual problems have been identified as areas requiring further research and development. The Vacumto-dir Transition and the magnetic lattice are two most critycal areas requiring near term effort. The success of the design ls highly dependent on these elements.

The mechanical mounts and slewing system needs a great deal of design optimization if the desfred high slewing rates are to be realized. System weight and vibration are seen as problems that will require a substantial development effort.

There are no technical reasons to doubt that a successful development can be achieved. No new materials are required. Existing technologies can be extended to meet the Identified needs of the program. 


\subsection{INTRODUCTION}

The object of this conceptual design study is the selection and Integration of sultable design concepte which can provide a very rapid and accurate deflection of a high-iatensity pulsed-beam of high-energy electrons. The performance objective for the bean director ls to accurately direct the electron beam to any target spot within a 60 degree half-angle cone and to be able to rapidly tedirect it to ang new target spot within this cone in a Eew teaths of a second. High. accuracy and high slewtng rates are required. It is desirable to keep the weight and size of the beam director at a minimum to achieve the desired high slewing rates with a minimum drive power.

The use of a system of permanent magnet devices for bending and focussing the electron beam is a way to minimize weight and to sfmplify the design. Alternative complicated electro-magnetic systems and their assoctated power supplies, cooling systems and electrlcal connections seem very difficult to accomodate in a director subfected to high rotational accelerations.

Since the system must be designed to handle electrons of some high energy yet to be determined (probably well in excess of $10 \mathrm{MeV}$ ), the dipole bending magnets and quadrupole focussing magnets must have fairly high magnetic flelds. The conceptual design should approach the limts of technology. The best available materlals and methods for achieving the objectives of the conceptual design should be explored.

The conceptual design must provide an exft window or transition for bringing the electron beam out of the vacuum system into the alr. It also must have an accurate electromagnetic steering system for providing prectse 
adjustments in the final exit angle of the beam. This steering system must he able to compensate for small afning errors and to cancel the adverse effects of mechanlcal vibrations.

The study effort was divided Into three tasks:

Conceptual Design

Iteison

Final Report

The first of these tasks, Conceptual Design, consisted of selecting likely concepts and configurations and performing those engtneering calculations required to evaluate the suitability of these concepts. The conceptual design engineering work included the following segments of the electron beam director:

- Magnet lattice

- Vacuum Rotary Joints

- Vaculum-to-Air Transition

- Vernier SteerIng System

- Mechanicel Hount and Slewing System

- Radiation Effects

- Estimates of Size, Weight and Cost 


\subsection{CONCEPTUAL DESIGN}

The conceptual design for the beam director is shown in Figure 2.0-1. This director consiats of a palr of magnetic bending and focusaing systems which are trounted and supported on rotatable structures. The direction of deflection of the beam 16 dependent upon the orlentation of the bending system and the angle of bend of each system. Since each bending syatem is supported on 1ts own totatable structure, the orfentation can be continuously cbanged to provide any desired resultant direction of the exit beam within a limited cone angle equal to twice the bend angle of each gystem.

The conceptual design shown fin Figure 2.0-1 also 1llustrates the detive systems for rotating the two structures and certain necessary auxtliary equipment. Most important of the auxlllary itemg are the Vacum-to-Ait Transition Section and the Vernier Steerfing System. The Interfor of the bending and focussing systems is held at a moderately high vacuum by a suitable set of vacuum pumps. A high vacuum in this beam transport system is a necessity and, therefore, the rotary joints must be vacuum tight. Since the electron beam must come out of the evacuated bending system and enter the atmosphere, a sultable transition vacuum/air section 18 required. This transition section has vaculm on one side and atmospheric pressure on the beam exit side. It must be nearly vacum tight and must be provided with sufficient vacuum pumpling capability to handle any leaks which may be Inherent in the system. The transition section must also be nearly transparent to the high energy electron beam because any signiflcant blockage of

*approximately $10^{-3}$ torr 

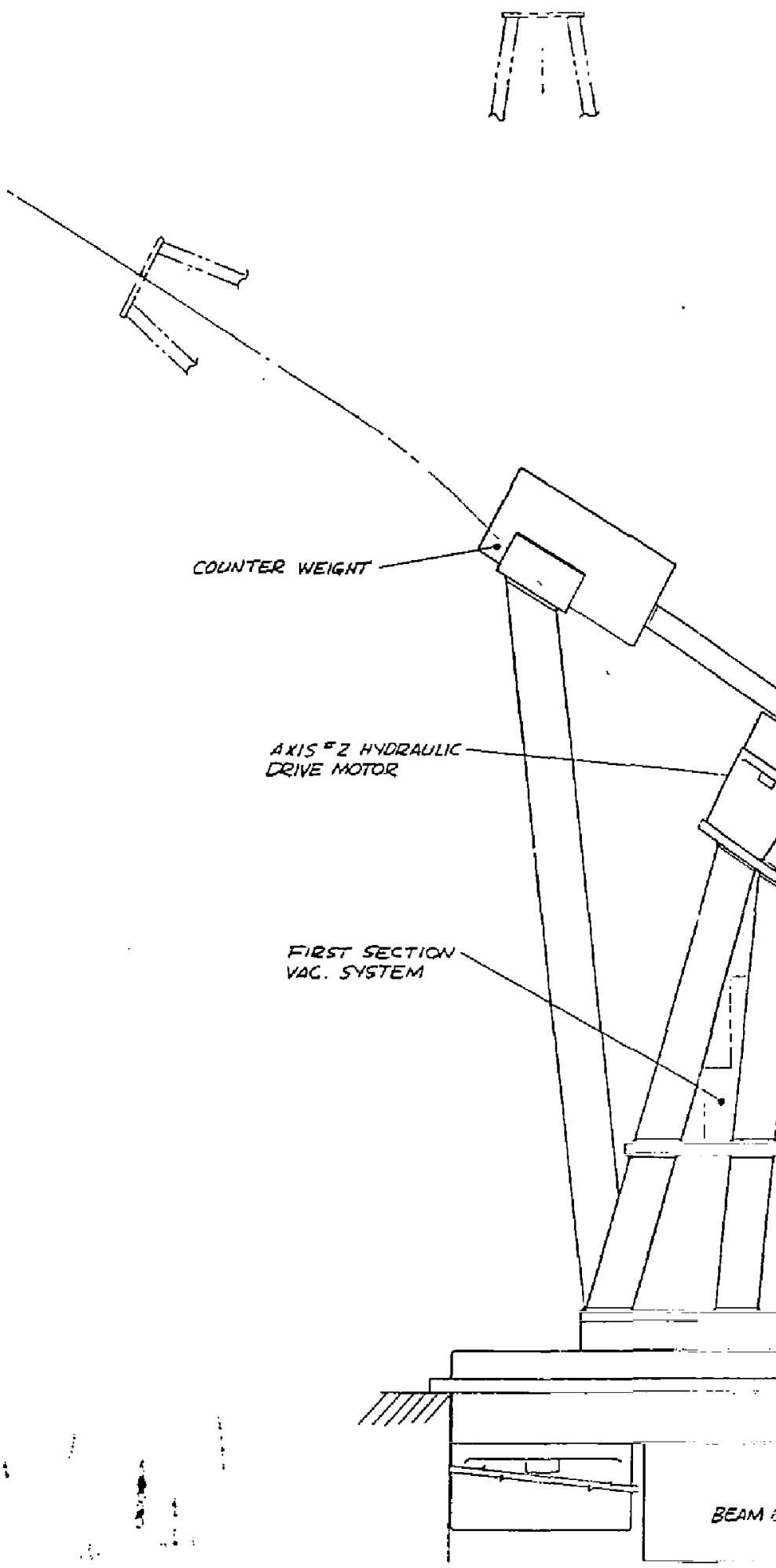
the beam will cause an extremely high heat laad. The heat load is potentlally high enough to melt or otherwise destroy most materials.

The final element at the exit of the beam director is a Vernier Steering System. This system consigts of electrical coils designed to give sufficient magnetic fleld to bend the beam slightly in any desired direction. This system w1l provide "fine tuning" of the beam exit angle and must have very rapid response so that it may be useful in cowpensating for any residual vibration in the mechanical structure.

The direction of the beam leaving the beam director is dependent upon the rotation of the bending systeme as 11lustrated in Figure 2.0-2.

Rotation of the upper structure through an angle $a_{2}$ about Axls 2 produces angular displacement $\theta$ measured downard along a meridian through the 2 axis (Axis 2). This rotation $q_{2}$, also produces the $B$ component of azimuthal displacemeat, $\emptyset$, about the $:$ axis. The rotation of the lower structure through an angle $a_{1}$ about Axis 1 produces additional aztmuthal displacement. The total azimuthal displacement, 0 , is given by

$$
\theta=\alpha_{1}+\beta=\alpha_{1}+\tan ^{-1}\left(\frac{\sin \alpha_{2}}{\cos \gamma\left(\cos \alpha_{2}+1\right)}\right)
$$

Where $Y$ is the bend angle for a single bending system.

The downward displacement, $\theta$, is given by

$$
\theta=\frac{\pi}{2}-\sin ^{-1}\left(\sin Y\left(1+\sin \gamma\left(1-\cos a_{2}\right)\right)\right)
$$

Selecting $\gamma$ having a value of 30 degrees gives

$$
\begin{aligned}
& \theta=a_{1}+\tan ^{-1}\left(\frac{2}{\sqrt{3}} \frac{\left(\sin a_{2}\right)}{\cos \alpha_{2}-1}\right) \\
& \theta=\frac{\pi}{2}-\sin ^{-1}\left(.75-.25 \cos a_{2}\right)
\end{aligned}
$$

The value of $\theta$ will vary from 0 to 60 degrees $(\pi / 3)$. The value of 0 will cover a fu1l 360 degrees $(2 \pi)$. 


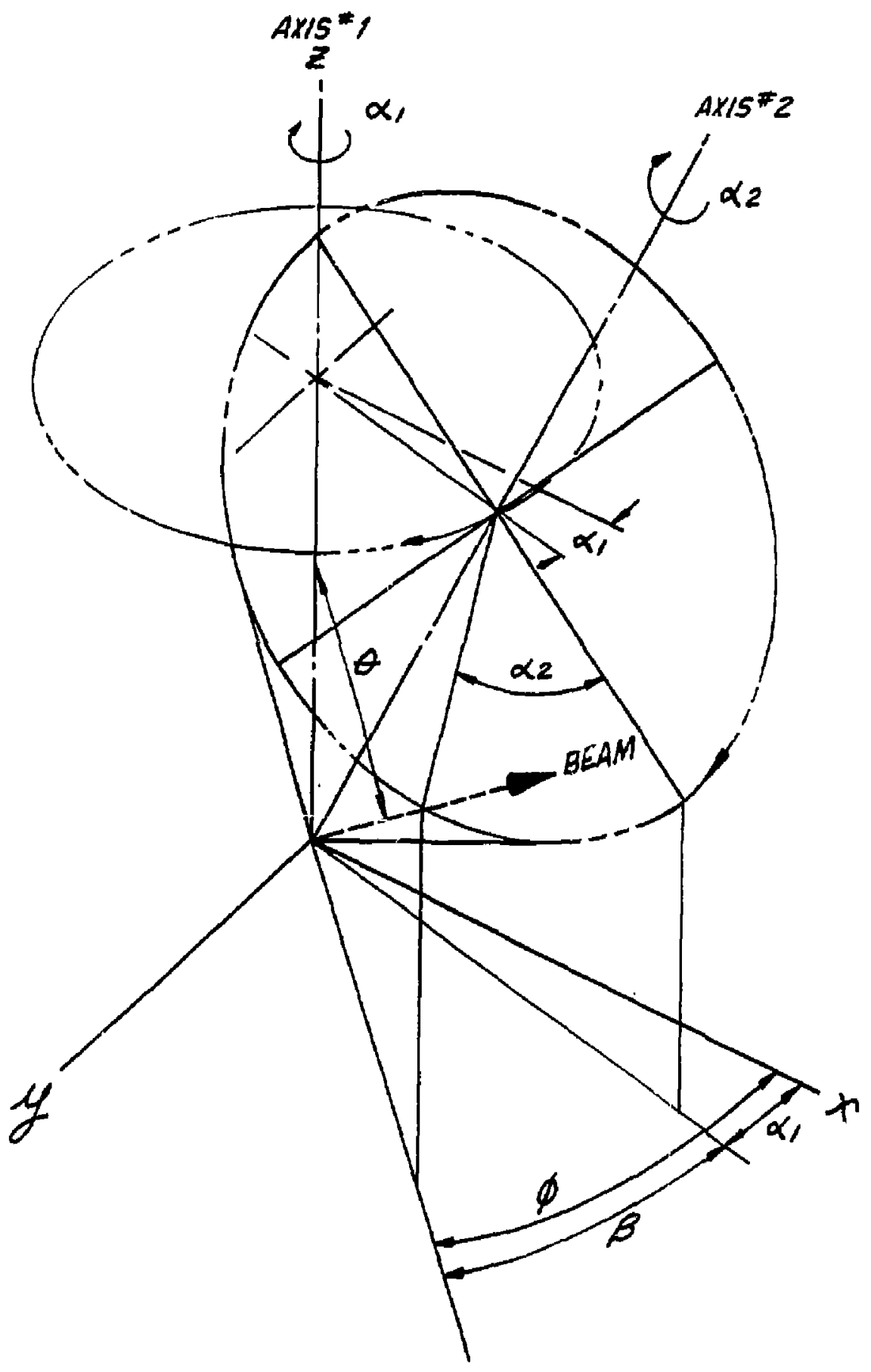

FIGURE 2,0-2 BEAM DIRECTION IN SPHERICAL COORDINATES $\theta$ AND $\phi$ SHOWS DEPENDENCE ON ROTATION $a_{1}$ AND $a_{2}$ 


\subsection{Magnet1c Lattice}

The high energy electron beam is bent and focussed by a sertes of permanent magnet dipules and quadrupoles. These must be arranged to produce an achromatic bend and sufficient focussing to keep the beam size small enough to avoid collistons with the wall of the beam tube. The magnetic strength required is dependent upon the beam energy and the desired radius of curvature in the bending section. A characteristic value for the bending and focussing system is the magnetic rigidity of the electron beam. This value can be expressed as the Br product where $B$ is the magnetic fleld in tesla and $I$ is the radius of curvature in meters. The $B r$ value may be tound from

$$
B r=\left[\begin{array}{ll}
T & (T+2 H o
\end{array}\right]^{\frac{1}{2}} \div 300
$$

Where $I$ and wo are respectively the kinetic energy of the electrons and Its Iest mass, both in MeV. For highiy relativistic electrons, the $\mathrm{Br}$ value approaches a linear function of the kinetic energy.

In order to achleve a bend wh a radius of curvature of one meter, the required magnetic fleld is as follows:

\begin{tabular}{rc} 
T, MeV & B, Tesla \\
\hline 10 & .035 \\
50 & .168 \\
100 & .335 \\
200 & .668 \\
500 & 1.668 \\
1000 & 3.335
\end{tabular}

Achleving a field much in excess of 1.67 tesla will be difficult using permanent magnets; to a radius of curvature greater than one meter will be requited if a beam of energy greater than $500 \mathrm{MeV}$ is desired. 


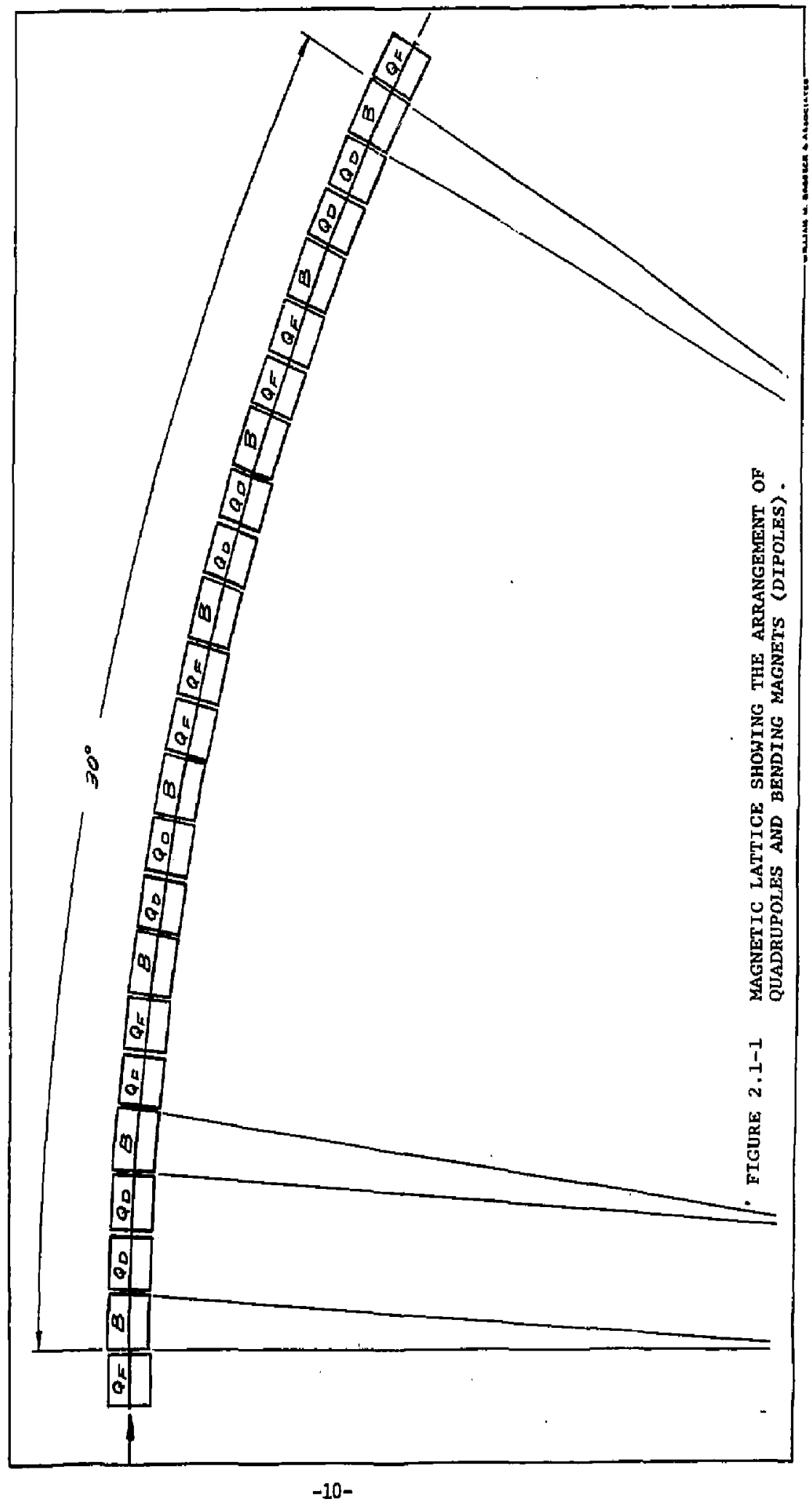


The best energy for which a beam director should be designed is not known, but the conceptual design can, in principle, be applfed to any energy value w1thin the range of 10 to $1000 \mathrm{MeV} . *$ W1thout loss of general1ty, a spectfic example can be selected for lllustration of the basic concepts. The speciflc example will use a Br value of 1.666667 tesla meters. The general arrangement of dipoles and quadrupoles is shown in Figure 2,1-1, where a $30^{\circ}$ bend is achieved using an array of 8 dipoles and 16 guadrupoles grouped 10 four identical combinations or cells, a cell consists of 4 quadrupoles and 2 dipoles arranged with mirror symetry to gfve a focus, bend, defocus, defocus, bend and focus. This arrangement of elements to make up a single cell can be made to produce a $90^{\circ}$ phase advance per cell if the magnetic properties and timensions are properly selected.

The combination of mirror symmetry and $90^{\circ}$ phase advance per cell will glve this syctem the advantage of maintaining a tightly focussed beam and wll assure acceptable achromatfcity. The total bend of $30^{\circ}$ will have a $360^{\circ}$ phase advance.

A basellae design case was selected for which the dipoles occupied one-third of the length alrng the beam path. For this baseline case, the dipole strength was selected to glve a bending radius of curvature of one meter and a bend angle per dipole of $3.75^{\circ}$. The quadrupoles occupying two-thirds of the length had their gradient set to achieve a $90^{\circ}$ phase advance.

The method of calculation involved a decermination of the matrix elements for the focugsing and defacussing quadrupoles and performing the necessary matrix moltiplication to find the overall transfornation matrix for a beam transversing the bending system. A simple program was set up so 
that a number of trial calculations could eas1ly be made and that after the baseline case was calculated, numerous alternative cases would be easily examined.

The matrix analysis was made for the vertical plane so that the dipole regions could be treated as drift spaces. The transport matrix equations are then as follows:

For focusaing quads

$$
\left|y^{\prime}\right|=\left|\begin{array}{cc|c|}
\cos \theta & \frac{1}{k} \sin \theta \\
-k \sin \theta & \cos \theta
\end{array}\right| x\left|\begin{array}{c}
z_{1} \\
y_{1}^{\prime}
\end{array}\right|
$$

For drift

$$
\left|\begin{array}{l}
y \\
y^{\prime}
\end{array}\right|=\left|\begin{array}{ll|l}
1 & s \\
0 & 1
\end{array}\right| \begin{aligned}
& y_{1} \\
& y_{1}^{\prime}
\end{aligned} \mid
$$

For defocussing quads

$$
\left|\begin{array}{l}
y \\
y^{\prime}
\end{array}\right|=\left|\begin{array}{cc}
\cosh \theta & \frac{1}{x} \sinh \theta \\
x \sinh \theta & \cosh \theta
\end{array}\right| x\left|\begin{array}{c}
y_{1} \\
y_{1}^{\prime}
\end{array}\right|
$$

Where $\mathrm{K}=\sqrt{\frac{\mathrm{G}}{\mathrm{Br}}}$ and $\theta=\mathrm{K} \ell$

$$
S=\text { Drift length }
$$

G = Magnetic gradient

$B=$ Magnetic fleld

$r=$ Bend radius

$\ell=$ Length of quadrupole

$y, y^{\prime}=$ Particle coordinate and angle 
For the baseline case, the magnetic properties and dimensions for the dipoles and quadrupoles were found to be as follow:

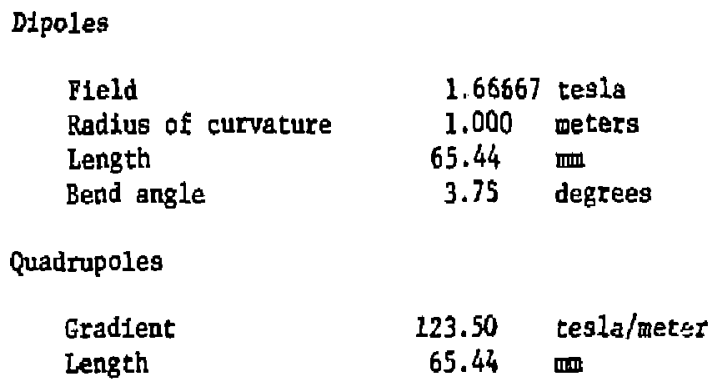

This gives a total length for a $30^{\circ}$ bending section of 1.57 meters.

A vartety of design approaches for the dipoles and quadrupoles were examined, ${ }^{*}$ Designs using permanent magnets are desirable. Designs using permanent magnets are clesrly possible at the field and gradient values required. Designs using soft iron pole tips and iron flux return paths appear to require slightly less pemanent magnet materfal than do ironless deglgns. However, designs uging soft iron are heavier overall and appear to present some sertous fringe fleld problems which are avoided in the ironless design.

The Ironless designs for dipoles and quadrupoles are based on the desfgn methods given by Klaus Halbach, LBL8906. His method assumes that a linear superposition of fields is valid. This ts a reasonably valid assumption for most rare-earth-cobalt magnets and for some oriented ferrites. An fmportant criteria for validity of this assumption is that the demagnetization curve for the permanent magnet must be linear tver the range of $B$ values used 1.0 the design and that the value of intrinsic magnetization, $\mathrm{B}-\mathrm{H}$, be nearly constant over the design range.

*The selected lattice has separated focussing and bending elements. Lattices with combined functions were analyzed and found to be less desirable than those with separated functions. 
F1gures 2.1-2, 2.1-3, and 2.1-4 show demagnitfation curves for some rare-earth-cobalt candidate material.* of thege, the Crucore 30 has the highest Bxh product which is conventionaliy used as a figure-of-merit for permanent magnets. However, the demagnitiagtion curve is not linear over the entire quadrant. The intrinsic magnetization $(B-B)$ remains nearly constant for a whlle but 1 d drops off rather sharply above $5 \mathrm{k} 0 \mathrm{e}$. The Crucore 26 has a lower Bxh product but has much better linearity than the Crucore 30 . There are other rare-earth-cobalt waterlals such as Crucore 18 which have excellent linearity with a high residual induction $B_{F}$.

F1gure 2.1-5 shows the demagnetization curve for an orfented ferrite material, Ferrimag $7 B$, which has very good linearity making it a good candidate for an ironless dipole or quadrupole of the style analyzed by Halbach. The ferrites have much lower regldual induction than the rare-earth-cobalt mocerials but they are much less expensive and they have lower density. For an electron beam with an energy less than $200 \mathrm{MeV}$, this Ferrite matertal would be a very good canoildate.

The ironless design concept for the quads and dipoles use 16 trapazoldal pieces arranged as shown in Figure 2.1-6. Each plece is magnetized in the direction shown with an arrow. The magnetic field in the gap is given by Halbach as follows:

$$
\begin{aligned}
& \text { Dipole } B=.974 \mathrm{~B}_{\mathrm{I}} \ln \left(\frac{\mathrm{r}_{2}}{\mathrm{r}_{1}}\right) \\
& \underset{\text { Quadrupole }}{B\left(\mathbf{r}_{1}\right)=1.875 \mathrm{~B}}\left(1-\frac{\mathrm{r}_{1}}{\mathrm{r}_{2}}\right)
\end{aligned}
$$

*The three matertals shown here are given merely as examples of the kinds of properties available. Sin. 2 new and stronger materlals are continuously belng developed, there 18 no doubt that stronger permanent magnet materials w11 be avallable in the future. 


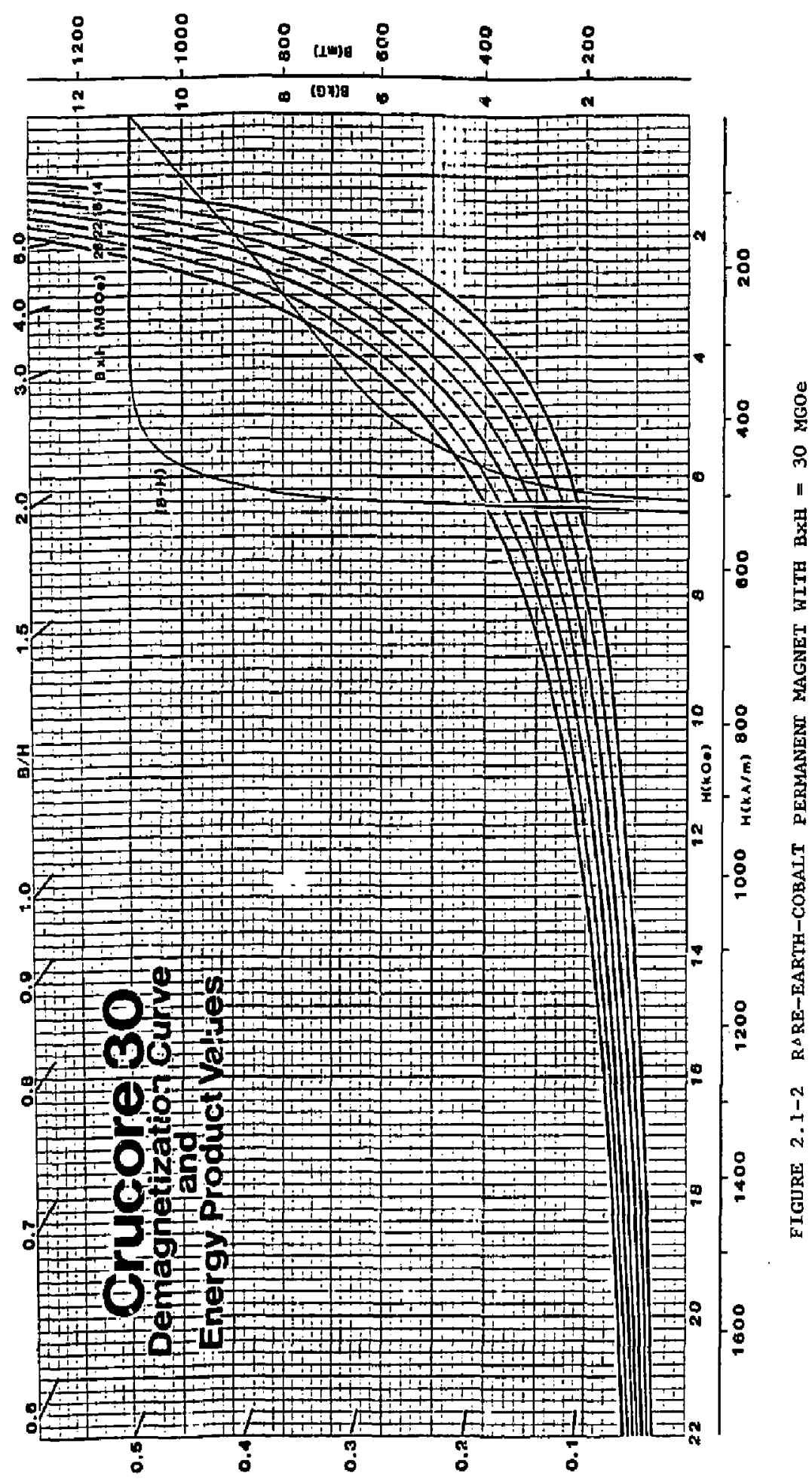




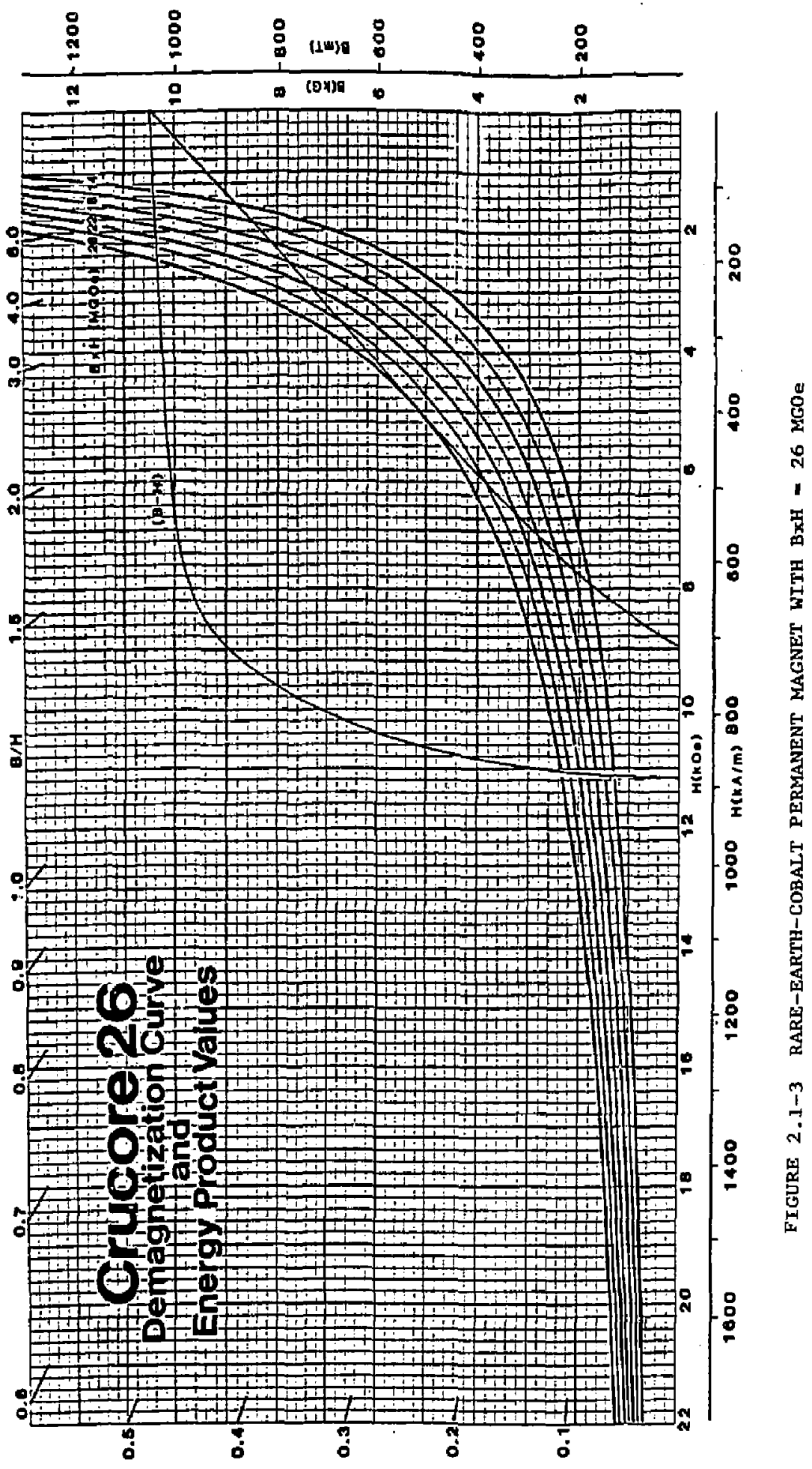




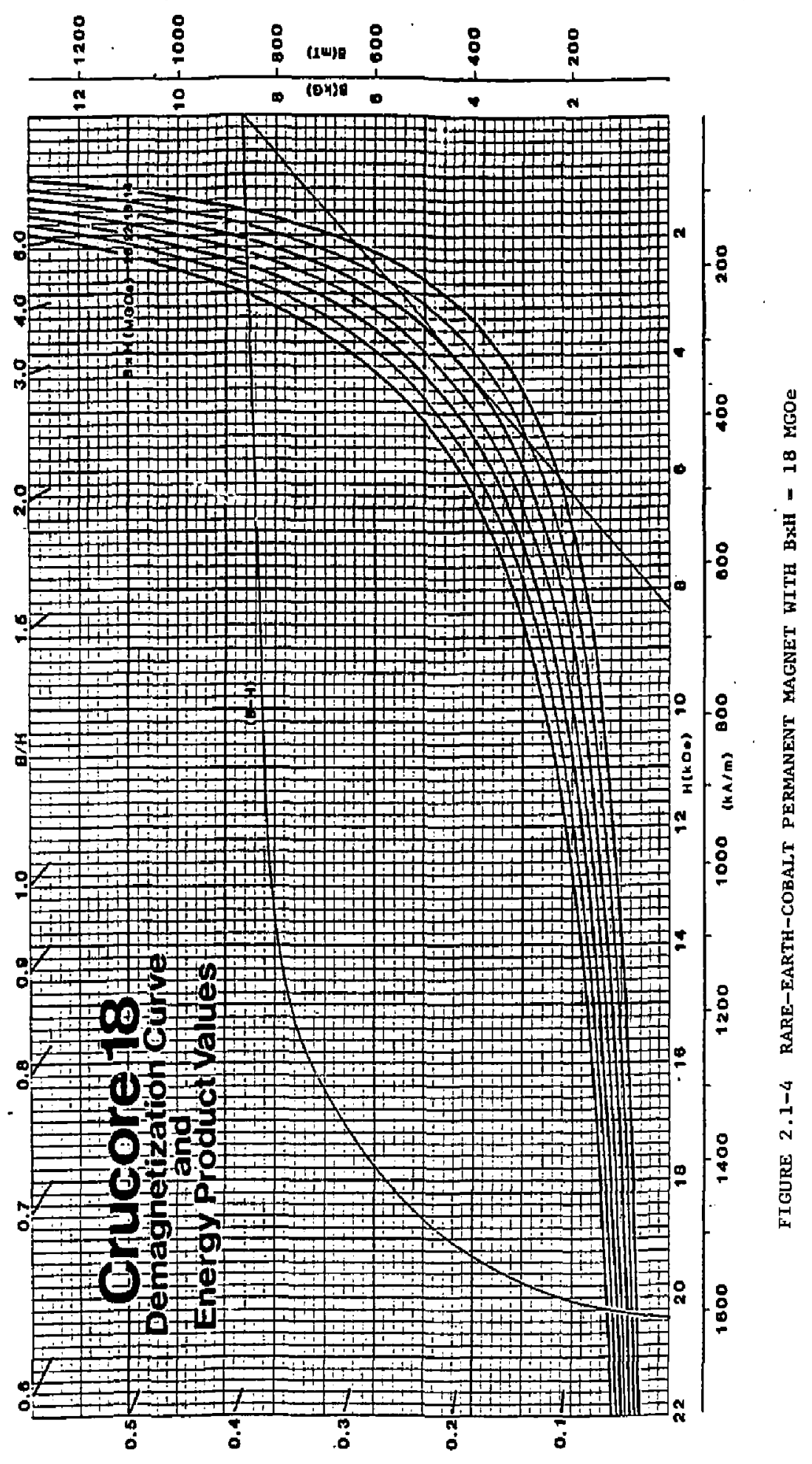




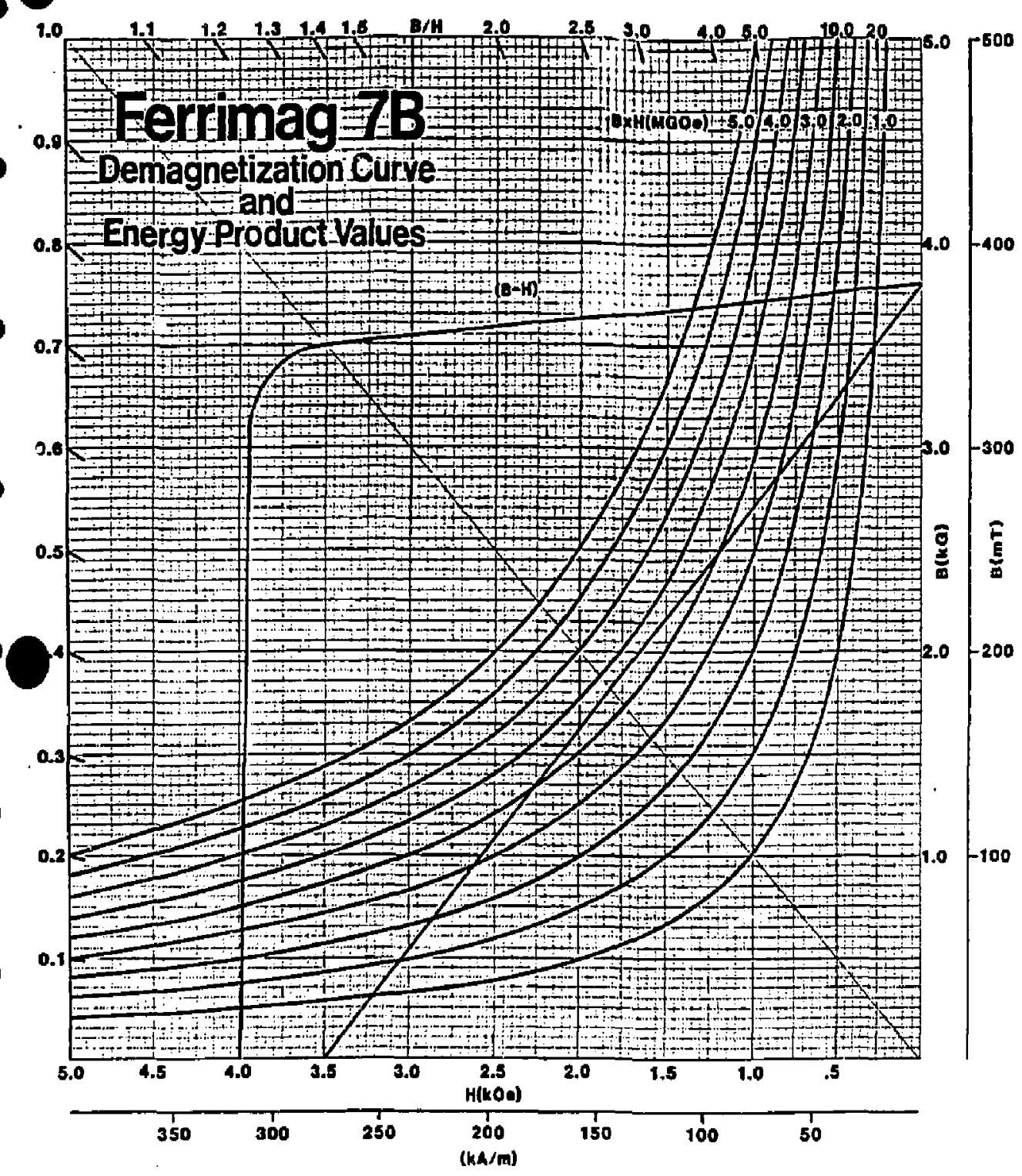

FIGURE 2.1-5 ORIENTED FERRITE PERMANENT MAGNET

WITH $\mathrm{BxH}=3.3, \mathrm{MGOe}$ 


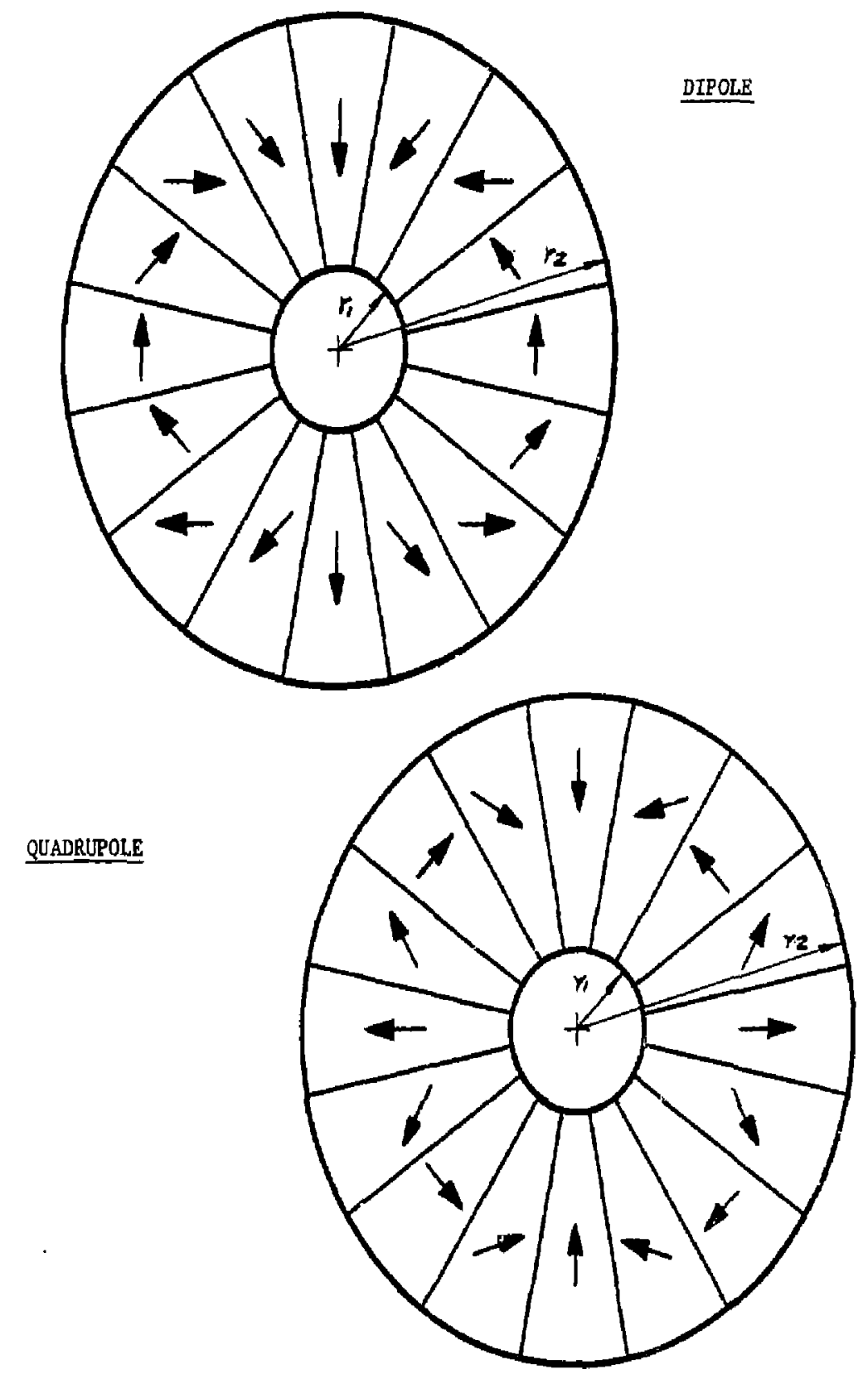

FIGURE 2,1-6 PERMANENT MAGNET DTPOLE AND QUADRUPOLES 16 TRAPEZOIDAL PIECES MAGNETIZED IN DIRECTION OF ARROWS 
Where $B_{T}=$ residual induction (from demagnetization curve)

$r_{1}$ a radi1 shown in F1gure 2.1-6

Using these equations for dipoles and quadrupoles of the baseline lattice structure having an inside radius $r_{1}=11 \mathrm{~mm}$ and using Crucore 26 , * the outside radil and weights were found as follows:

Dipole

$$
\begin{aligned}
& r_{1}=11.0 \mathrm{~m} \\
& r_{2}=56.13 \mathrm{~m} \\
& 1^{2}=6.5 .44 \mathrm{~m} \\
& W_{t}=5.17 \mathrm{~kg}
\end{aligned}
$$

Quadrupole

$$
\begin{aligned}
& I_{1}=11.0 \mathrm{~m} \\
& I_{2}=35.48 \mathrm{~m} \\
& 1=65.44 \mathrm{~m} \\
& W_{t}=1.94 \mathrm{~kg}
\end{aligned}
$$

Ways of decreasing the welght of the baseline case were explored.

It was clear from the Ealbacb equations for 1ronless permanent magnet dipoles and quadrupoles, that the weight of these elements depend upon thetr field strengths and upon the properties of the material used. Since the materfal selected for the baseline case is nearly the optinum available material, the only practical way to reduce the weight is to lower the magnetic fields. Lowering the fleld requires an increase in the length of the bendiog elements. This can be done without fncreaging the total length of the system if the quadrupoles are shortened while the dipoles are lengthened.

A varlety of cases were calculated to assure that suitable beam transport properties were retalned as the dipole lengths were 1ncreased. F1gure 2.1-7 shows the decrease in dipole weight assoctated with decreasing

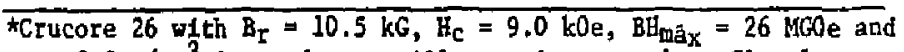
$\rho=8.3 \mathrm{~g} / \mathrm{cm}^{3}$ is used as an illustrative example. Clearly, alternat fves with greater Ineerity are avallable. 
1ts fleld. To retain proper focussing, the quadrupole strengths had to be increased which required an increase in the quadrupole weights. These Increases are also shown. By comparing total weights and component diameters, a compromise case was selected. The compromise case is nearly at the minimum weight condition, but has a more favorable diameter ratio than does the minimum welght case. Table 2.1-1 shows the various properties of the calculated cases.

The values snowa are for a $500 \mathrm{MeV}$ syster. Obviousiy, these would not be the proper speciftc values of a diverter for an electron beam of different energy. However, the method of calcuiation and the general design concept is valid and can be applied to other energy levels. For Inwer energy, it would be possible to use systems which are shorter and lighter in weight.

At $50 \mathrm{MeV}$ (aTA, for example), a beam diverter using ferrite appears to be practlcal. Such a systen could use a peak field in the dipole of about .141 tesla and have an overall length of each $30^{\circ}$ bend of about 1.57 meters.

The bending system using permanent magnet material requires sufficient structural strength and rigidity to accurately hold the permanent. magnet wedges. A sultable concept for assembly of system and for support of the evacuated beam tube is also required. Figure 2.1-8 shows a conceptual design for this support. It consists of two curved stainless steel shell pieces bolted together at the midplane. This concept allows the bending system to be assembled in two halves which are assembled around the beam tube. This outer shell is then attached to larger frame type structure wilch transmits the dynamic loads assoclated with rapld slewing of the system. An evacuated stainless steel inner tube of about $1 \mathrm{~mm}$ thickness carries the return current. 
TABLE 2.1-1 SUMMAY OF LATTICES CALCULATED

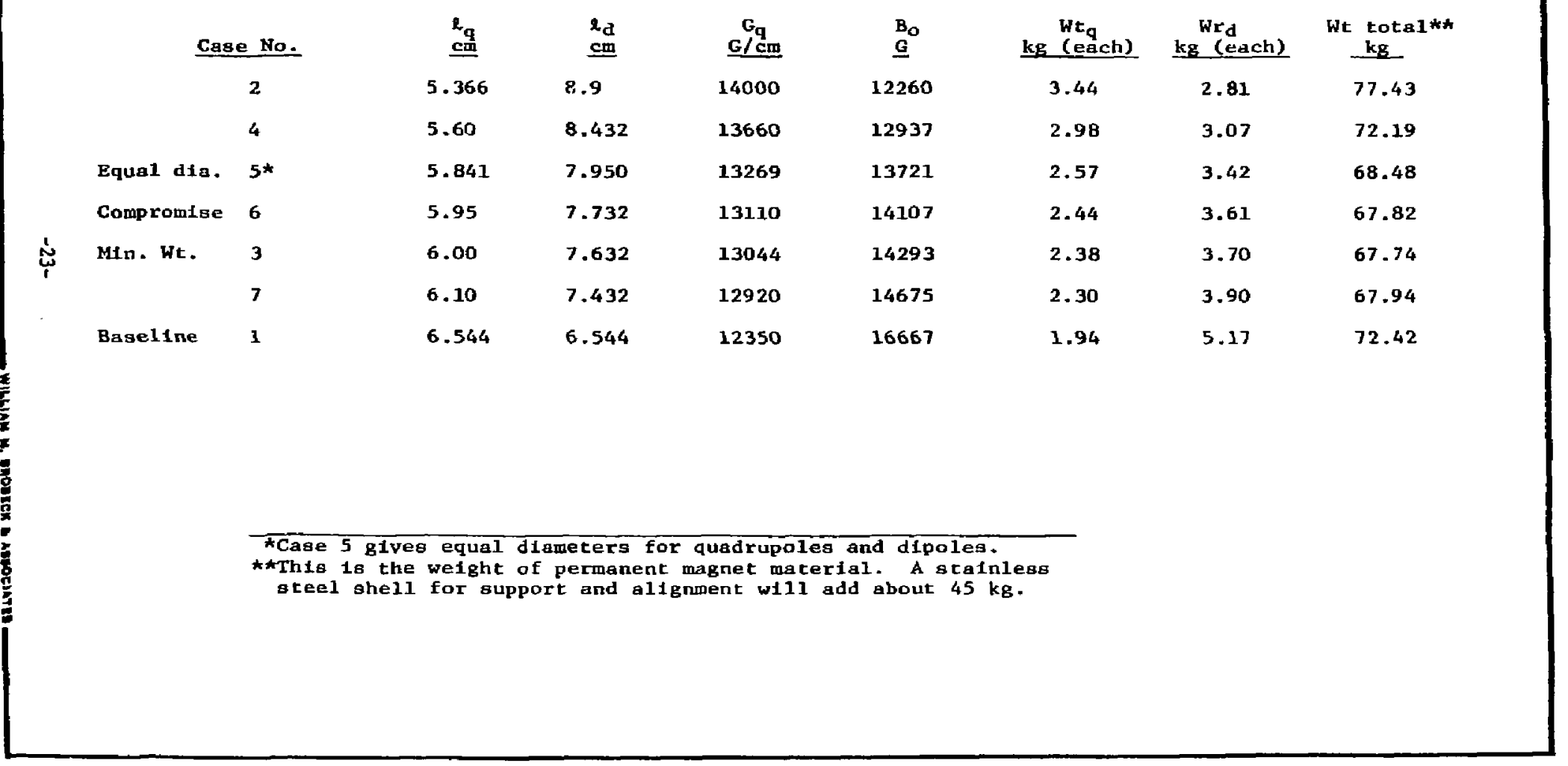




\subsection{Vacuum Rotary Jotnts}

The bending systems for the electron director must be rotated about their respective inlet axes in order to alter the direction of the exit beam. There are two bending systems in the conceptual design. Each of these must be provided with a support structure and a means for rotating these two structures about their axes. A rotary joint is required for each of these systems. These joints must provide accurate alfgnment and must be nearly vacuum tight. It is belfeved that it will be imposstble to provide an absolutely vacuum tight seal, so some provision for minimizing leakage and for vacuum pumping must be provided.

In detail, the requirements for the rotary vacuum joints are the following:

1. Permit $360^{\circ}$ rotation.

2. Provlde a good vacuum seal with a very low leakage rate.

3. Provide adequate vacuum pumping ports for rough vacuum purping and for high vacum pumping.

4. Kaintain accurate alignment, concentricity, and angularity,

5. Provide sufficient strength and rigidity to withstand the dynamic loads due to $180^{\circ}$ slewing in .3 seconds.

6. Provide electrical current path for beam tube counter current.

7. Provide support for the drivs mechanism with strength for the rotational torque reaction and for the overturaing torque.

The rotary vacuum joint for the upper bending sectlon must carry all the dynamic loads of the upper structure. These loads include the dypamic force assocfated w1 th rotation about Axis 2 (See Figure 2.0-1) and also those assoclated with rotation about Axls 1. The rotational load due 
to turnlng about Axis 2 can be nesrly balanced. However, the rotation about Axris I produces dynamic load that must be carried by this rotary joint. A Alscussion of these dynamic loada will be made fa the sectlon dealing with mechanical mounts and slewing system. Suffice 1t to say here that the dynamic losds on the upper joint are wuch lower than those on the lower joint because the loads on the upper joint superimpose upon those assoctated with rotation of the lower structure about axis 1 .

A conceptual design for the upper rotary vacuum folnt is shown in Figure 2.2-1. The lower joint will be simflar but stronger. The vacuum seal and counter current flow paths are shown conceptually. The vacuum seal consists of 0 -rings with an intermediate pump-out port. The double 0-ring is for two-stage pumping, but three-stage pumping may prove to be necessary in practice. These seals must be lubricated to minimize friction and vacuun leakage.

The counter current flow path is through a spring loaded contactor connected to a squirtel cage conductor. This open squirrel cage also permits vacuum pumping. 


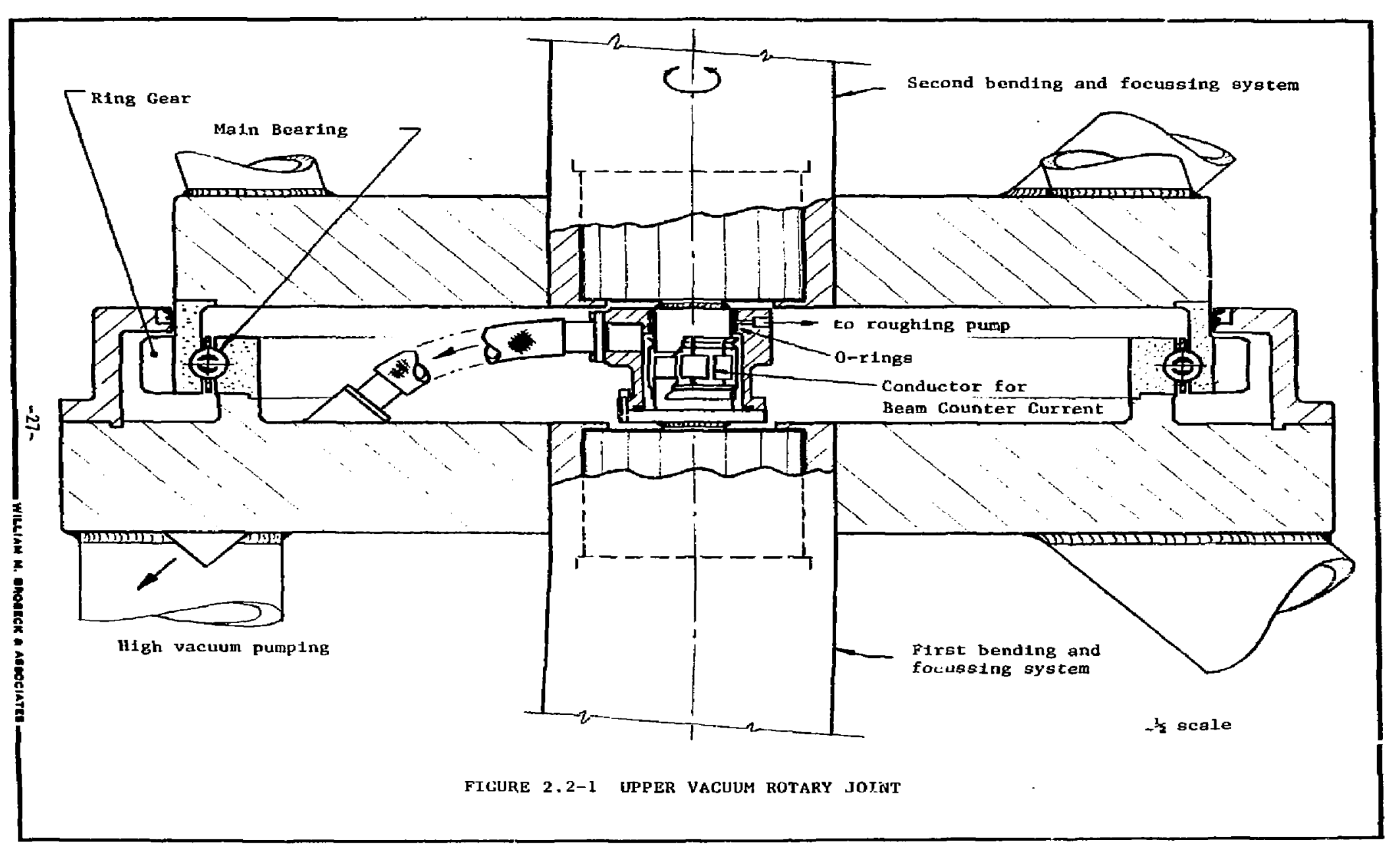




\subsection{Vacuum-to-Air Transition}

The electron beam is transported in a high vacuum and must be brought out of the director into the alr. An abrupt transition from vacumto-air is desired. A thin foll whdow strong enougi to withstand the differentlal pressure would provide an ideal trangition if it were sufficlently transparent to high energy electrons. Most potential foil materials will pecmit a very large percent of the electron beas to pass through; however, the very suall percent of the beam energy that is lost in the foil will deposit a great amount of thermal energy into the foil. If the energy is concentrated by a mall diameter beam, the local heating could be great enough to melt or otherwise destroy the foil through thermal shock and spallation.

To obtain a prelimfary evaluation of potential foll materials and to 1llustrate the severity of the thermal problem, a simple calculation of the heating of a wide range of materlals was made. For this calculation, the stopping range for $50 \mathrm{MeV}$ electrons and the $s_{4}$ ecific heats of the materials were assumed as congtants, independent of temperature. These calculations were made for a one-centimeter dlameter, $50 \mathrm{MeV}$ beam having $35 \times 10^{3}$ joules per pulse (based on ATA 70 ns pulse). Table 2.3-1 shows the results of these calculations.

As can be seen in this table, the temperature rise per pulse increases rapidly with an increase in atomic number. A single pulse passing through an aluminum foil would raise the temperature to a value more than three times its meltidg point.* A simple pulse through a copper foil would raise its temperature to almost six times fts melting point.*

\footnotetext{
Won-Inearity of the specific heat curve and latent heats of melcing are 1gnored so this is not absolutely accurate!
} 


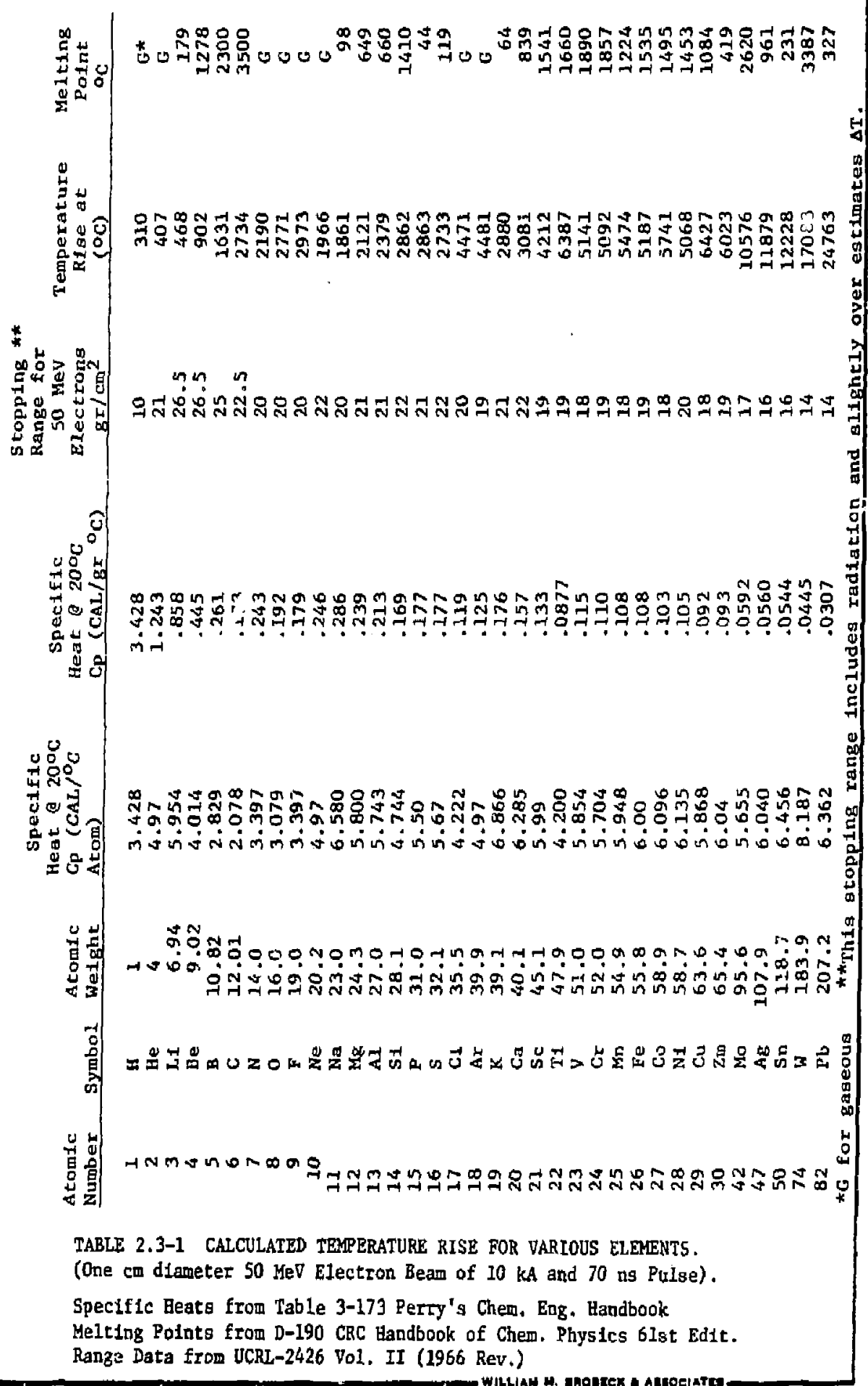


The only materials which appear to have any practical value as foll windows are thase using elements with low atomic numbers. Pure elements such as beryllium of carbon are obviously attractlve, although beryllium may present some special radiation problems. The high serength of beryllium and its light weight make it especially attractive. Combloatlons such as siliconcarbide or boron-carbide are also reasonable candidates,

The values in the table are for heating by a one centineter diameter 70 as beam pulse with $10 \mathrm{kA}$ of current at $50 \mathrm{MeV}$. These are the values expected for ATA. For higher beam energy, the thermal deposition will not change siguificantly. A shorter beam pulse, say 10 ns will have a very significant reduction in the heating per pulse. A 10 ns pulse at $10 k+11$ i cause one-seventh of the temperature rise of a 70 ns pulse. Reducti- * beam diameter will also have a significant thermal effect. Changlng the diameter from one centimeter to one-half centimeter will cause the temperature rise to increase by a factor of four (tgaoring non-linearity of the specific heat function).

Taking into account the non-linearitles of the spectific heats and assuoing that ATA pulses can be chopped to $10 \mathrm{~ns}$ and focussed to $4 \mathrm{~mm}$ diameter, the single pulse temperature rise for carbon and beryllium are calculated to be the following:

$$
\begin{array}{ll}
\text { For beryllium } & \Delta \mathrm{T}=654^{\circ} \mathrm{C} \\
\text { For carbon } & \Delta \mathrm{T}=1164^{\circ} \mathrm{C}
\end{array}
$$

For any case requiring a multitude of pulses closely spaced in time, the temperature rise would be excessive unless the material in the path of the electron beam 1s somehow renewed or cooled. The scheme considered for the

\footnotetext{
*Taking into account the non-1inearities of the specific heat results in a lower temperature rise than would otherwise result.
} 
conceptual design is one in which. the foil is rotated at a high speed to bring a new portion of the foll into the path of the beam for each pulse. Figure 2.3-1 shows the conceptual degign for the Vacuum-to-Aft Transition Section. A rotating disk is shown in the path of the beam. For a beam having a diameter of less than 5nm and pulsing in the frequency range from 1 to $33 \mathrm{kHz}$, a tangential speed of the disk at the beam spot of 150 meters per sec assureg that the disk bas travelled the full ofdth of the beam between pulses. This speed is subsonic and w1ll not cause excessive stress in a lightweight disk made of beryll1um.

The high rotational speed of the disk can be used to aid in purging air from the space around the disk. By sultable design, the rotating disk can be made to function as a turbo-molecular pump. A suitable roughing pump and pumping ports will be required.

of course, there are other possible schemes for a Vacuum-to-Air Transition Section. A larger diameter beam can be brought through a nonrotating foil. Such a beam can result from defocussing in the vacuum upstream of the foll and then be refocussed after passing chrough the foll. Refocussing the beam in alr or other gas at atmospheric pressure has potential problems which need to be studled.

At this time, the rotating fot1 concept appears to be a viable one which will add very liftle to the spstem moment of Inertia. 


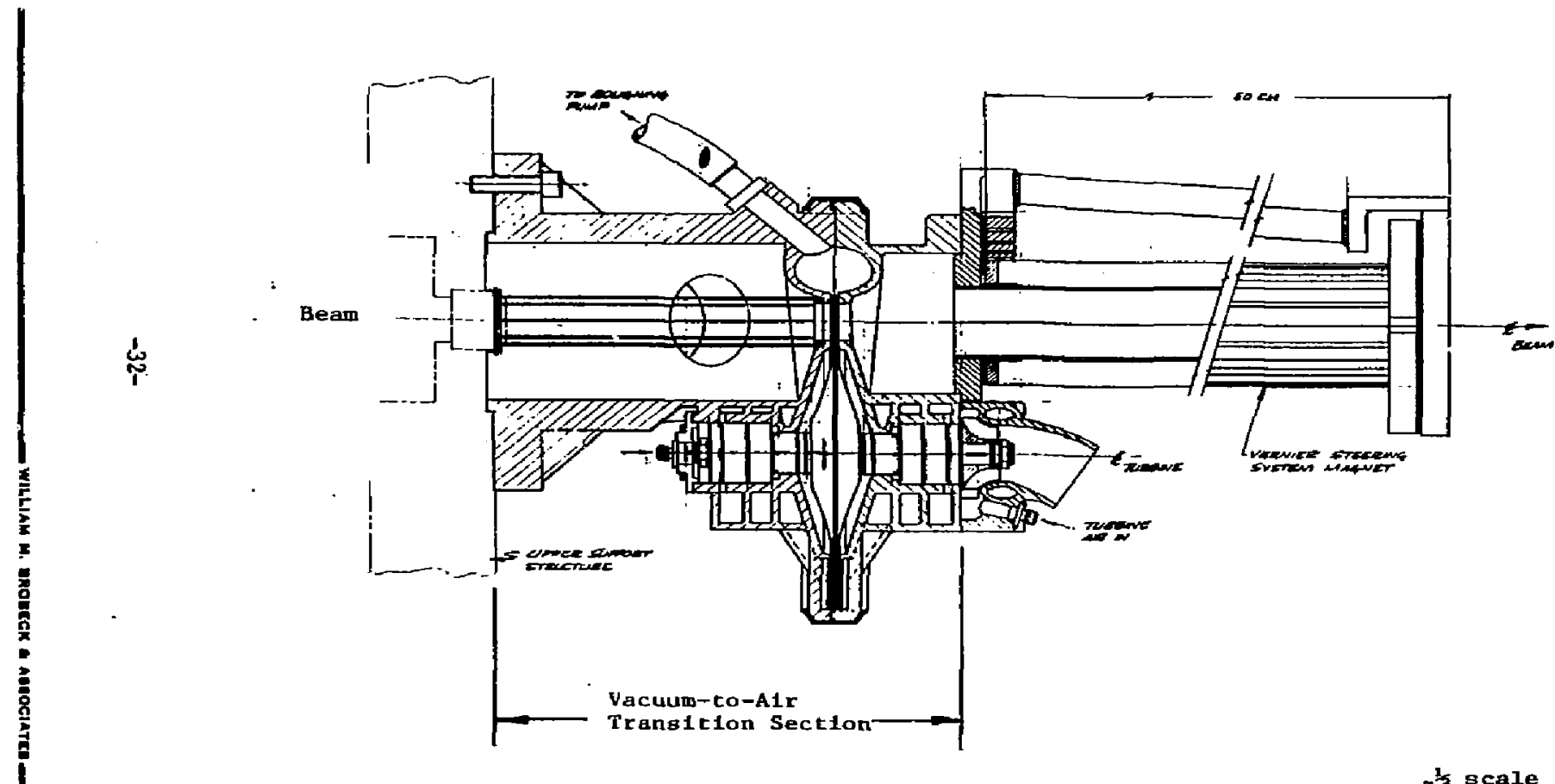

FIGURE 2.3-1 VACUUM-TO-AIR TRANSITION WITH HIGH SPEED ROTATING DISK. 


\subsection{Vernler Steering System}

A Vernier Steering System to provide electro-magnetic steering of the beam after it has traverged the Vacuum-to-Air Transition Section is desired. This steering system will provide "fine tuning" of the bear director to correct for vibration and small afming errors. The total correction to be provided is one degree. Th1s one degree bend can be in any direction. Thus, two sets of putually perpendicular colls are required. Figure 2.4-1 shows the Veraier Steering System mounted downstream of the Vacuum-to-Air Transition Section.

This system would be 500um long for a $500 \mathrm{Mel}$ beall and would be capable of giving a one degree bend. This bend wlll require a very modest magnetic field of $58.2 \mathrm{mT}$. A bend of one degree in a length of $500 \mathrm{~mm}$ will take the bean off the axts of the steering magnets a distance of $4.3 \mathrm{~mm}$. Thus, the magnetic field in the steering system must be fairly uniform. It was at first thought that a four-wire system could be used for the Vernler Steerfog System, but it doea not now appear that auch a siople system will provide a magnetic fleld of sufficient uniformity.

The system shown in the figure uses two sets of coils which are fnterwound. Each provide a nearly costae variation in amp-turns. Such a vartation will gfve good fleld uniformfty. Figure 2.4-2 showg the coll cross section. The colls labeled, $X$, operate on one power supply to give a very unlform vertical field. The colls labeled, $Y$, operate on a separate power supply to glve a very uafform horlzontal field. By adjusting the curcent in each set of coils, a resultant fleld of any direction and magnitude (Wtbin limits) can be obtained. The steering magnet concains no magnetic Iron and can be operated at high frequency with very little hysteretlc or eddy current losses. 



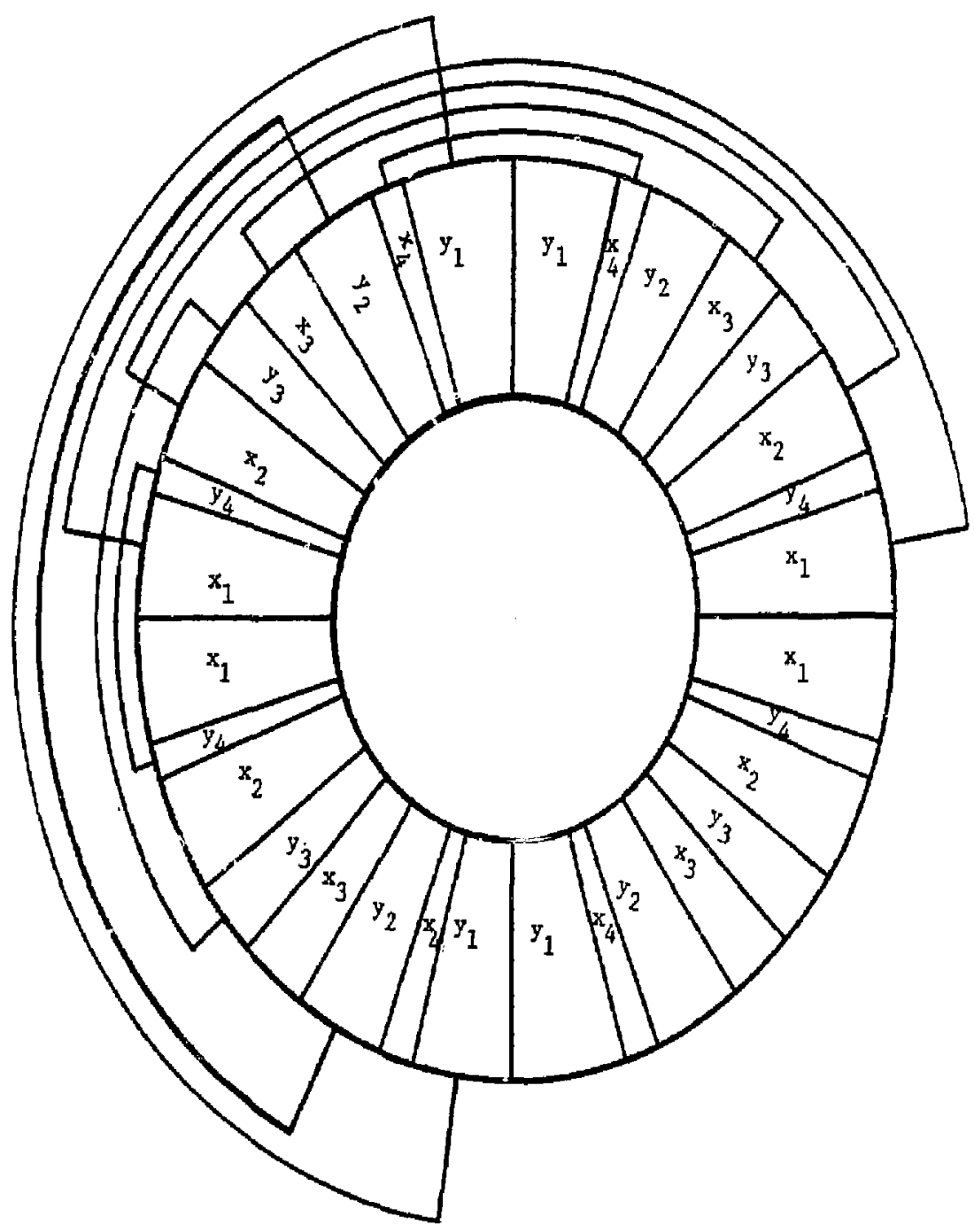

Horizontal deflection zolls $x_{1}, x_{2}, x_{3}$, and $x_{4}$ require one power supply and vertical deflection coils $y_{1}, y_{2}, y_{3}$, and $y_{4}$ another 


\subsection{Mechanical Mounts and Slewing System}

The "slewing system" refers to the structures and drive systems needed to point the beam. It's design and structural characterlstics afe discussed here. Gyrodynamlc losding calculations and structural response calculations are tllustrated and described.

The prinicpal beam director mechanical subasemblies are the two (2) $30^{\circ}$ bending magnet arrays used to point the beam, together with the necessary supporting structures, vacuum system Elements, and drive system elements. Hereafter, these w1ll be discussed as the "Axis 1 system" and the "Axis 2 System." Also, In refarring to the location of components along the bean line, "upstream" mearls towards the accelerator and "downstream" means towards the beam exit.

The Axis 1 Syetem attaches to the end of the Beal Transport System (BTS) and rotates about the BIS beam line which is coincident with "Axis 1." The downstream beam line out of the Axis 1 System 1s rotated $30^{\circ}$ with respect to Axds 1, and is coincident with "Axis 2." The upstream beam Line of the Axis 2 System coincides with the downstream beam line of the Axls 1 System. The downstream beam line of the Axis 2 System is rotated $30^{\circ}$ with respect to Axts 2. This general arrangement is illustrated on Figure 2.0-1. Ay is 1, coincident with the BTS beam 11ne, is a fixed datum axis. Axis 2, colactdent with the Axis 1 System downstream beam 1ine, is tilted $30^{\circ}$ with iespect to Axis 1 and rotates about 1 . The Axis 2 System "spins" about Axis 2 which in turns "whiris" about Axis 1. The Axis 1 System "whIrls" about Axis 1. 
The Axis 2 System is supported on a multi-load bearing normal to and centered on Axts 2, located at the downstream beam line exit from the Axis 1 System. The Axts 2 Systim is to be both dynamically and statically balanced for rotation about Axis 2. The distribution of mass and flexibility along Aris 2 in the vicirity of the transition section attachment may be selected to reduce siructural rotation at the beam line exit. The Axis 2 System Includes the transition section, means to pump the transition section, driye gear and support bearing, plus assoctated structure and balance veight. The Axis 1 System is supported on a multi-load bearing normal to and centered on Axis 1, located at the downgtream beam line exit from the BTS. This sytem may be "over balanced," to support the Axis 2 system in a way that compensates for both structural rotation and translation of the exit beam line at the transition section. Such ..echanical "tuning" is not free; it is associated with increased system weight and drive power. The Axis 1 System 1acludes the Axis 2 Syster drlve, means to pump the rotating vacuum joint, drive gear and support bearing, plus assoclated structure and balance velght.

\section{Degcription of Calculation Strategy}

Sketch DN132-165-1 (Appendix A) illustrates "pipe truss" representations for both the Axis 1 and Axis 2 Systems. Sketch DN132-165-1B (Appendix B) is a full-scale schematic of the rotary vacuun joint that supports the Axis 2 System. It shows a full sized multi-load support bearing and a general arrangement for a yacuum seal and electrical feed through. Neither drawing represents a Incommended mechanical design; it is too early In the degiga process for that. Both drawings were used as a basis for weight 
estimates, gyrodyaamic loading calculations, and structural regponse calculations. So Ear, only a "beam bending" type structural response has been estimated. Each supporting cruss is comprised of a circular pattern of eight (8) pipes that surround the $30^{\circ}$ bending magnet arrays. These pipes terminate in welded housings. Two sets of calculations were performed for assumed steel trusses and for assumed aluminum trusses. The axis 2 system used 3 inch diameter tubes with .094" wall thickness. The Axis 1 System used 6 Inch diameter tubes with .094 wall thickness,

Tables 2.5-1 and 2.5-2 sumarize the components and wefghts used for both the Axis 1 and Axis 2 Systems. In this study, the cargo component weights were not varied. The structure component weights, initially for steel, were then reduced by a factor of three to represent an aluminum structure. The Axis I System category titled "Balance, Vacuum System, Plus Other" was varfec to 1llustrate how the balance of the Axis 1 System affects the translation and rotation of the transition section, and the assoctated trade off with drive power.

A three (3) step process was followed in estimating dynamic structural response.

1. The non-axisymetric structures of DN132-165-1, 1B and Tables 2.5-1, 2 were divided into several mass stations for calculation of gyiodynamic lop's versus time. For this, the structures are assumed to be rigid.

2. Then, the peak value gyrodynamic loads were applied to simplified, flextble beam todel representations to calculate static structural deflections. The first mode yibration frequency was estimated from these static structural deflections and the model distribution of mass and inertia. 
TABLE 2.5-1

WEIGHT SUMQARY FOR AXIS 2 STSTEM

CARGO

$\frac{\text { St'l }}{\text { (lbs) }} \quad \frac{A l}{(1 \mathrm{bs})}$

Transition Section

11. $\quad 11$.

Roughing Punp

Yacuum Pư⿱⺈ \#1

Vacuum Pump $\# 2$

50. 50.

Bearing Ring and Gear

50.50 .

50.50.

Magnet Assembly

73.73.

$\underline{250.8} \quad \underline{250.8}$

$\begin{array}{lll}\text { Subtotal } & 484.8 & 484.8\end{array}$

STRUCTURE

\begin{tabular}{lcc} 
Transition Plate & 42.4 & 1413 \\
Structural Tubes and Stiffeners & 158.12 & 52.61 \\
Bese & $\underline{138.00}$ & 46.00 \\
\cline { 2 - 2 } Subtotal & $\underline{338.52}$ & $\underline{112.84}$ \\
\multicolumn{1}{l}{ TOTAL } & $\mathbf{8 2 3 . 3 2}$ & 597.54
\end{tabular}




\section{TABLE 2.5-2}

WEIGHT SUMMARI FOR AXIS 1 SYSTEM

CARGO

\section{Magnet Assembly}

Hydraulic Drive and Motor

Axis 2 Brg Inner Pace

Base Geat

$$
\frac{S t^{\prime} \ell}{(1 \mathrm{bs})} \quad \frac{\mathrm{Al}}{(1 \mathrm{bs})}
$$

$$
250.8 \quad 250.8
$$

125.

75. 75.

753.

$1203.8 \quad 1203.8$

STRUCTURE

Upper Brg Support

Center I Strut Support Plate

Tubes and Upper Structure

138. 46.

$82.5 \quad 27.5$

$218.35 \quad 72.78$

Tubes and Lower Structure

$1253.05 \quad 417.68$

$1691.9 \quad 563.96$

BALANCE, VACUUM SISTEM, PLUS OTHER

Case (1)
Case (2)
Case (3)

AXIS 2 SYSTEM

Total Case (1)

Case (2)

Case (3)

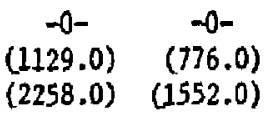

$823.32 \quad 597.54$

$3719.02 \quad 2365.30$

(4848.02) (3141.30)

(5977.02) (3917.30) 
3. The static responses were then multiplied by a dynamic multiplier hased on the grodynamic load signature as a function of time, and the natural periods of the flexible beam model representa:ions.

\section{Calculation of Gytodinimic Loads}

For the non-axisymetric structures conaldered here, it proved convenient to approach the computation of gyrodynamic loads in a very primitive way, i.e., many mass stations were set up, each 1dentified by axis position, radius normal to the axis, and angular orientation about the axis, The rigid body, absolute accelerations of these mass stations were ther calculated as functions of spin and whirl kinematics. The assoclated gee loadings wete then combined to find cross-section forces and moments, bearing loads, and drive torques.

The calculation model for station acceleration is 1llustrated on

Figure 2.5-1. The associated component acceleration equations are tabulated on Table 2.5-3. The derivation leading to these equations followed the format of a similar derivation ${ }^{*}$ involving constant whirl and spin velocities.

General integral equations for spin and whirl kinemarics are included on Table 2.5-3. For the calculated results here, it was assumed that spin and whirl yelocitles started at zero, fncreased at a constant rate, and then decreased at the same negative constant rate untfl the velocities were again zero. This scheduling is buflt into the equations stated on Table 2.5-4. The numerical results of this study assume a $180^{\circ}$ total angle change in $300 \mathrm{~ms}$ for each axis. The peak angular velocity is $200 \mathrm{rpm}$. The angular accelerations are $\pm 139.6 \mathrm{rad}$ per sec per sec.

In calculating Axis 2 system loads, the tilt angle, $\theta$, was set at $30^{\circ}$. The calculation Input for thịs system is Iisted on Table 2.5-5. 


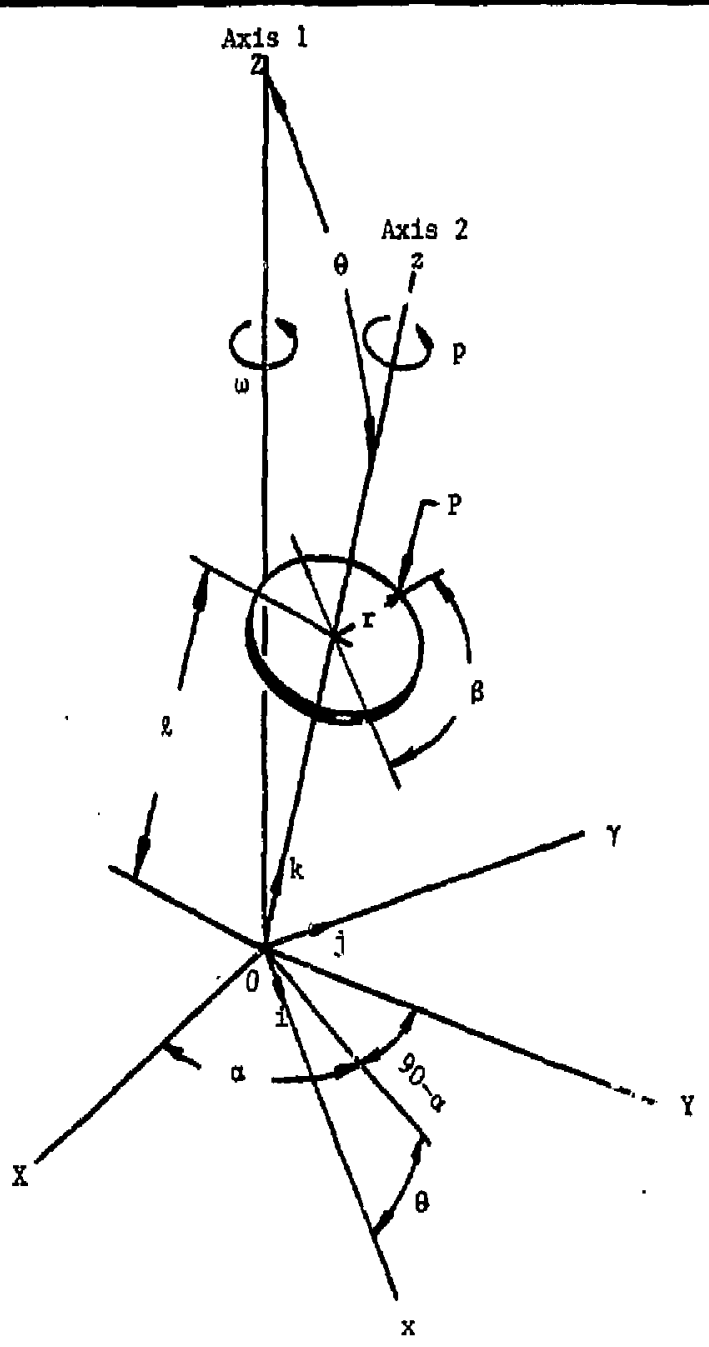

FIGLRE 2.5-1 GYRODYNAMIC LOAD CALCULATION SCHEMATIC DIAGRAM

$X, Y, 2$ Fixed coordinate system

$x, y, z$ Whirling coordinate system; Plane 20z ( $20 \mathrm{x})$

Whitls about Axis 1 with angular velocity w.

a Is the whirl angle. $\theta$ is the tilt angle between Axis 1 and 2.

$1, f, k$ Unit vectors along $x, y, z$

\&, $I, B$ Cootdinates of point $P$ that spins about Axts 2 with angular veloctty $p$. $1 \mathrm{~s}$ the spin angle wh thespect to whirling plane $z 0 x$. 
TABLE 2.5-3

ABSOLUTE ACCELERATION OF POINT P

IN WHIREING XYZ COORDINATE SYSTEM

See Figure 2.5-1

$$
\begin{aligned}
& a=a_{1} 1+a_{2} j+a_{3} k \\
& a_{1}=-r(p+w \cos \theta)^{2} \cos \beta-\ell w^{2} \sin \theta \cos \theta-r(\dot{p}+\dot{c} \cos \theta) \sin \beta \\
& a_{2}=-r(p+w \cos \theta)^{2} \sin \beta-\cos ^{2} \sin ^{2} \theta \sin \beta+r(\dot{p}+\dot{w} \cos \theta) \cos \beta \\
& +\lim \sin \theta \\
& \theta_{3}=-r\left(2 p \omega+\omega^{2} \cos \theta\right) \quad \sin \theta \cos \beta-\ell \omega^{2} \sin ^{2} \theta-r \dot{\omega} \sin \theta \sin \beta
\end{aligned}
$$

Where

$$
\begin{array}{ll}
\omega=\int_{0}^{t} \dot{j} j t & p=\int_{0}^{t} \dot{p} d t \\
a=a_{0}+\int_{0}^{t} \omega d t & B=\beta_{0}+\int_{0}^{t} p d t
\end{array}
$$

For the present estimites, equal constant values were assumed for both whirl and spin angular accelerations. 


\section{TABLE 2.5=4}

WEIRL AND SPIN KIMEMATICS

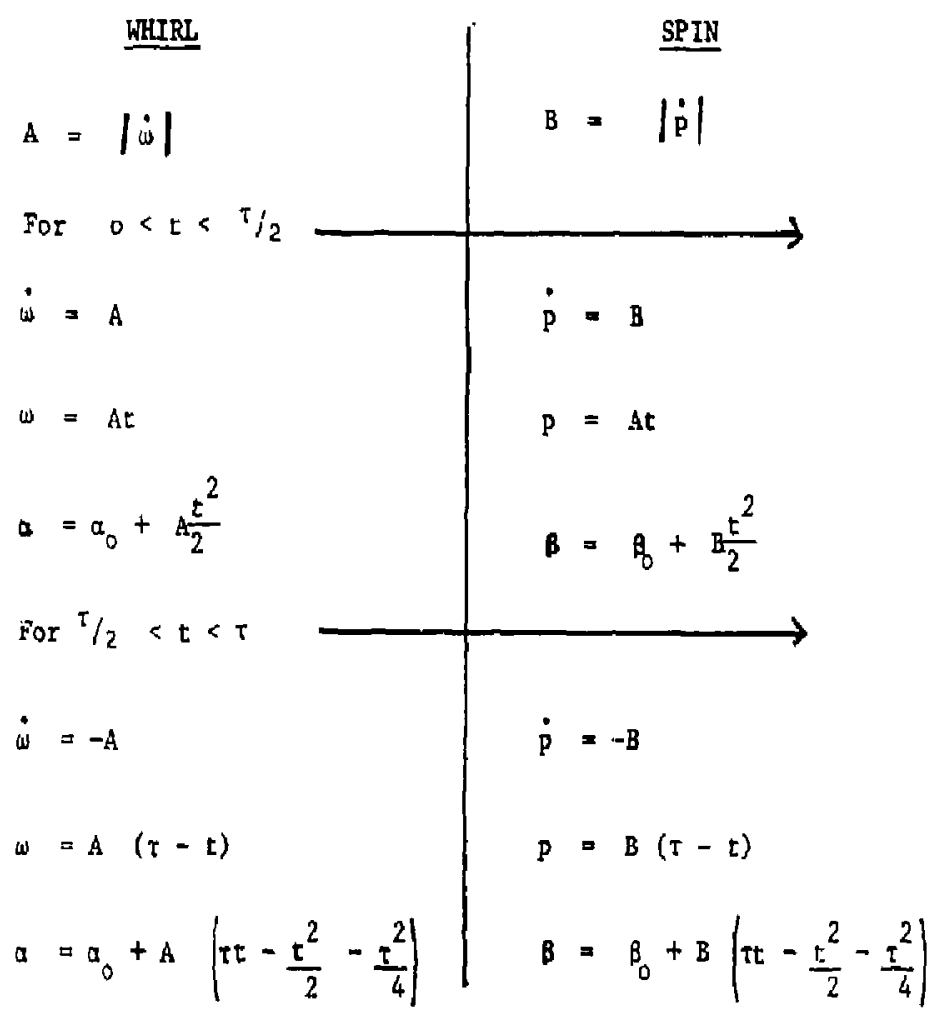


Length Radius

rient. Weight.

1. $97.67 \quad 17.80+0.00 \quad 11.00$

$\begin{array}{lllll}2 & 92.59 & 14.90 & +0.00 \quad 131.28\end{array}$

3. $66.3915 .40^{\circ}+0.00 \quad 5.95$

$\begin{array}{lllll}4.69 .89 & 14.29+24.50 \quad 6.15\end{array}$

$\begin{array}{llll}5 & 68.19 & 11.19+48.55 \quad 6.55\end{array}$

$\begin{array}{lllll}6 & 69.09 & 6.18 & +73.40 & 6.95\end{array}$

$7 \quad 69.59 \quad 0.55+180.00 \quad 7.10$

$\begin{array}{llll}8 & 69.09 & 0.18 & +286.60 \quad 6.95\end{array}$

$9 \quad 68.19 \quad 11.19+311.45 \quad 6.55$

$10 \quad 66.89 \quad 14.29+335.50 \quad 6.15$

$\begin{array}{lllll}11 & 65.69 & 0.00 & +0.00 \quad 30.00\end{array}$

$\begin{array}{lllll}12 & 39.69 & 0.100 & +0.00 & 226.87\end{array}$

$\begin{array}{lllll}13 & 83.89 & 1.97+180.00 & 10.90\end{array}$

$\begin{array}{llll}14 & 64.19 & 16.80+180.00 \quad 6.00\end{array}$

$15 \quad 49.59 \quad 12.70+180.00 \quad 4.40$.

$\begin{array}{lllll}16 & 70.07 & 10.85 & +180.00 & 2.12\end{array}$

$\begin{array}{lllll}17 & 31.15 & 0.00 & +0.00 & 73.00\end{array}$

$\begin{array}{lllll}18 & 70.19 & 20.80+180.00 & 50.00\end{array}$

$19 \quad 59.69 \quad 22.40+180.00 \quad 50.00$

$20 \quad 63.89 \quad 11.50+180.00 \quad 50.00$

$\begin{array}{lllll}21 & 65.26 & 4.04 & +0.00 & 125.40\end{array}$

$\overline{823.32 \mathrm{lbs}}$

\section{ALOMINUM STRUCTURE}

$\begin{array}{rrrrr}\text { No. } & \text { Length } & \text { Radius } & \text { Orient. } & \text { Weight } \\ 1 & 97.67 & 17.80 & +0.00 & 11.00 \\ 2 . & 92.59 & 14.90 & +0.00 & 85.46 \\ 3 & 66.39 & 15.40 & +0.00 & 1.98 \\ 4 & 69.89 & 14.29 & +24.50 & 2.05 \\ 5 & 68.19 & 11.19 & +48.55 & 2.18 \\ 6 & 69.09 & 6.19 & +73.40 & 2.32 \\ 7 & 69.59 & 0.55 & +180.00 & 2.37 \\ 8 & 69.09 & 6.18 & +296.60 & 2.32 \\ 9 & 68.19 & 11.19 & +311.45 & 2.18 \\ 10 & 66.89 & 14.29 & +335.50 & 2.05 \\ 11 & 65.69 & 0.00 & +0.00 & 10.00 \\ -12 & 39.69 & 0.00 & +0.00 & 117.42 \\ 13 & 83.89 & 1.97 & +180.00 & 3.63 \\ 14 & 64.19 & 16.80 & +180.00 & 2.00 \\ 15 & 49.59 & 12.70 & +180.00 & 1.47 \\ 16 & 70.07 & 10.85 & +180.00 & 0.71 \\ 17 & 31.15 & 0.00 & +0.00 & 73.00 \\ 18 & 70.19 & 15.80 & +180.00 & 50.00 \\ 18 & 59.69 & 17.40 & +180.00 & 50.00 \\ 20 & 63.89 & 11.50 & +180.00 & 50.00 \\ 21 & 65.26 & 4.04 & +0.00 & 125.40\end{array}$


For the Axis 1 System, the tilt angle, $\theta$, was set at $0^{\circ}$. Since the Axis 2 System is both dynamically and statically balanced, it is represented by a single mass station in the Axts 1 System computations. The calculation input for the Axts 1 System is listed on Table 2.5-6.

\section{Static Structural Response Calculacions}

Figure 2.5-2 11lustrates the model used. to estimate flexible static load response for the entire system. The forces $T_{1}, V_{1}$, and $M_{1}$, are in the Axis 2 System which is considered here to be a stralght beam-like structure tilted at $30^{\circ}$ along Axis 2. The actual Axis 2 structure on one side tilts off Axis 2 and is balanced both statically and dynamically by additional structure that is oppositely located. The forces $T_{2}, V_{2}$, and $M_{2}$ are in the Axls 1 System pipe truss which 1 s considered to be a curvea beam-like strucrure. The balance weight, $W_{B}$, exerts a tension on the mass station located where the Axis 2 System attaches to the Axis 1 Systen at the location of the Axis 2 support bearing. The equations that ccrrespond to this representation are illustrated on Figure 2.5-3.

The lengths listed in Table 2.5-5, for the Axis 2 gyrodynamic calculation, are measured from the intersection of Axis 2 and Axis 1. The CG of the Axis 2 System was computed to be at 60 1ns. All items located at a length greater than 60 ins were lumped to produce end loads $\mathrm{T}_{\text {so }}, \mathrm{V}_{\text {so }}$, and $M_{\text {so }}$. All items located at less than 60 ins. were lumped into the station at the Axis 2 support bearing.

The lengths listed in Table 2.5-6, for the Axis 1 gyrodynamic calculations, are measured from the bottom support bearing fllustrated on DN132-165-1. In terms of this 11st, the heavy base Items 12 to 19 are 
TABLE 2.5-6

AXIS 1 GYRODYNAMIC CALCULATION STATION INPUT

STEEL STRUCTURE

$\begin{array}{rrrr}\text { No. } & \text { Length } & \text { Radius } & \text { Drient. } \\ 1 & 69.50 & 15.60 & +0.00 \\ 2 & 66.43 & 13.80 & +0.00 \\ 3 & 65.30 & 8.40 & +180.00 \\ 4 & 63.00 & 13.97 & +108.36 \\ 5 & 56.00 & 20.39 & +68.58 \\ 6 & 49.00 & 23.99 & +33.54 \\ 7 & 46.70 & 24.20 & +0.00 \\ 8 & 49.00 & 23.99 & +326.46 \\ 9 & 56.00 & 20.39 & +291.42 \\ 10 & 63.00 & 13.97 & +251.64 \\ 11 & 29.50 & 4.80 & +0.00 \\ 12 & 4.30 & 24.20 & +180.00 \\ 13 & 4.30 & 24.20 & +135.00 \\ 14 & 4.30 & 24.20 & +90.00 \\ 15 & 4.30 & 24.20 & +45.00 \\ 16 & 4.30 & 24.20 & +0.00 \\ 17 & 4.30 & 24.20 & +315.00 \\ 18 & 4.30 & 24.20 & +270.00 \\ 17 & 4.30 & 24.20 & +225.00 \\ 20 & 33.50 & 4.50 & +0.00 \\ 21 & 77.00 & 12.50 & +180.00 \\ 22 & 98.50 & 30.37 & +180.00 \\ 23 & 98.50 & 30.50 & +0.00\end{array}$

\section{ALIMINUN STRUCTURE}

Height

75.00

200.70

28.63

2B. 20

27.22

26. 28

25.92

26.28

$\div 7.22$

2 E. 20

Q2. 50

254.92

253.63

250.69

247.87

246.79

247.87

250.69

255. 63

1BB. 10

125.00

$0.00=$ No balance weight 823.00

$\overline{3718.34} 1 \mathrm{bs}$

$\begin{array}{rrrrr}\text { No. Length } & \text { Radius } & \text { Orient. } & \text { Height } \\ 1 & 69.50 & 15.60 & +0.00 & 75.00 \\ 2 & 66.43 & 13.80 & +0.00 & 108.70 \\ 3 & 65.30 & 9.40 & +190.00 & 9.54 \\ 4 & 63.00 & 13.97 & +108.36 & 9.40 \\ 5 & 56.00 & 20.39 & +68.58 & 9.07 \\ 6 & 49.00 & 23.99 & +33.54 & 8.76 \\ 7 & 46.70 & 24.20 & +0.00 & 9.54 \\ 8 & 49.00 & 23.99 & +326.46 & 8.76 \\ 9 & 56.00 & 20.39 & +291.42 & 9.07 \\ 10 & 63.00 & 13.97 & +251.64 & 9.40 \\ 11 & 29.50 & 4.80 & +0.00 & 27.50 \\ 12 & 4.30 & 24.20 & +180.00 & 147.72 \\ 13 & 4.30 & 24.20 & +135.00 & 147.29 \\ 14 & 4.30 & 24.20 & +90.00 & 146.31 \\ 15 & 4.30 & 24.20 & +45.00 & 145.57 \\ 16 & 4.30 & 24.20 & +0.00 & 145.01 \\ 17 & 4.30 & 24.20 & +315.00 & 145.37 \\ 18 & 4.30 & 24.20 & +270.00 & 146.31 \\ 19 & 4.30 & 24.20 & +225.00 & 147.29 \\ 20 & 33.50 & 4.50 & +0.00 & 188.10 \\ 21 & 77.00 & 12.50 & +180.00 & 125.00 \\ 22 & 98.50 & 30.37 & +180.00 & 0.00 \\ 23 & 98.50 & 30.50 & +0.00 & 598.00\end{array}$




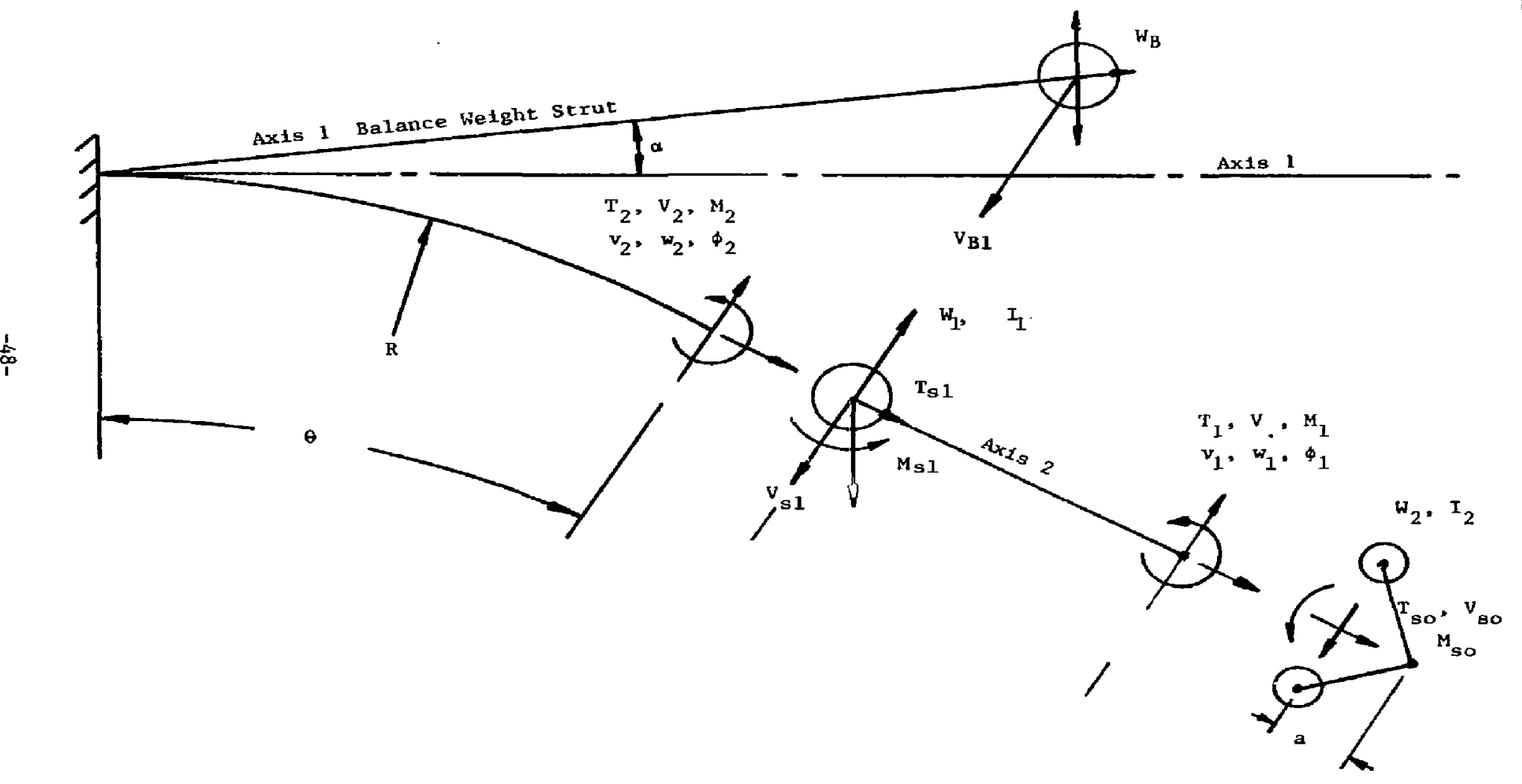

FIGURE 2.5-2 6 DEGREE OF FREEDOM AXIS 1 SYSTEM SCHEMATIC 
1gnored and Item 22 representing $3 / 4$ of the magnet array mess also is ignored. These items are very heavy, mostly centered about Axis 1 , and are located at the base of the cantilever system in very stffe structure. They will not load the Figure 2.5-2 flexible cantilfver beam system to a significant degree. All the other items are lumped at the Axis 2 support bearing.

The flexib1a length of the Axis 2 straight beam representation 1s 61.44 ins. The flexible length. of the Axis 1 curved beam representation corresponds to a radius of $300 \mathrm{~cm}$ (118 ins) swept over a $30^{\circ}$ arc. The beam crossection properties cortespond to the sections 1llustrated on Figure 2.5-4. The dimensions were scaled from the intermedlate circular tube truss patterns 11lustrated on DN132-165-1. All influence coefficlent computations include terms for transverse shear in addition to beam bending.

The coefficient matrices and loading vectors for laput to the equations 1llustrated on Figure 2.5-3 are 1isted on Table 2.5-7 for the steel structure, and on Table 2.5-8 for the aluminura structure. Loading vectors are 1llustrated for three difference Axis 1 balance weights. The loading level corresponds to 44 gees at the Axis 2 mass station at the tip and 19 gees at the Axis 2 support bearing.

A prior preliminary study was made of the exis 2 System as a 3-station, 6-degree of freedom cantilever beam. That model is illustrated on Figure 2.5-5, and the associated equation matrix is illustrated on Figure 2.5-6. These early calculations are of interest only insofar as they 11lustrate considerable stiffness in the Axis 2 System when it is cantilevered from a datum plane. Much larger deflections are calculated when the Axis 2 System is cantllevered off the Axis 1 System because the comblned 


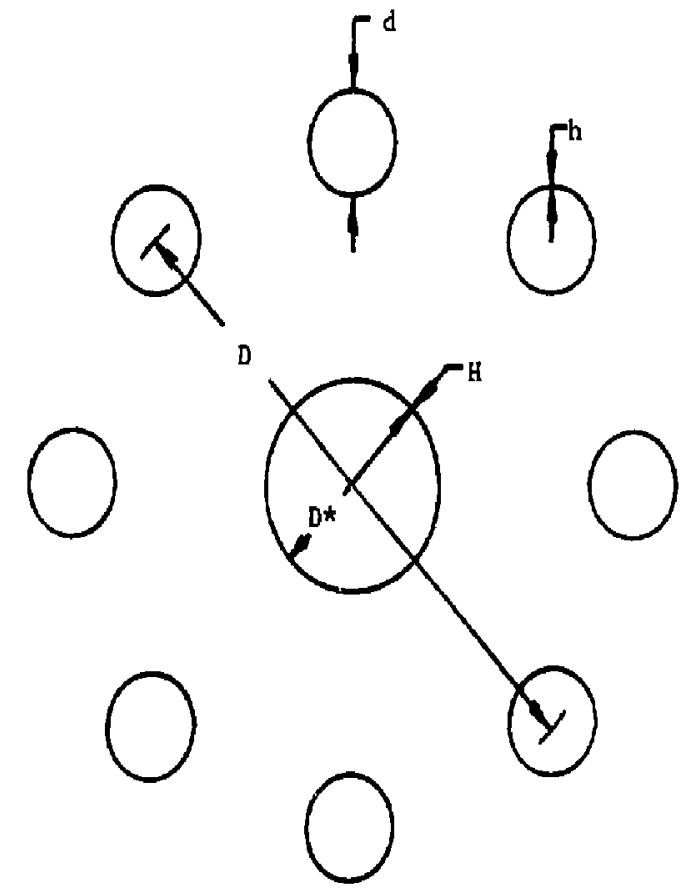

\begin{tabular}{|c|c|c|c|c|c|}
\hline Axis 2 & $D$ & $\mathrm{~d}$ & $\mathrm{~h}$ & $\mathrm{D}$ & $\mathrm{H}$ \\
\hline Axis 1 & 16 & 3 & .094 & 3.54 & .094 \\
\hline
\end{tabular}

FIGIRE 2.5-4 STRUCTURAL CROSS SECTIONS FOR ESTIMATION OF BEAM LIKE FLEXIBILITIES 
TABLE 2.5-7

STEEL STRUCTURE

MATRIX COEFFICIENTS AND LOAD VECTORS

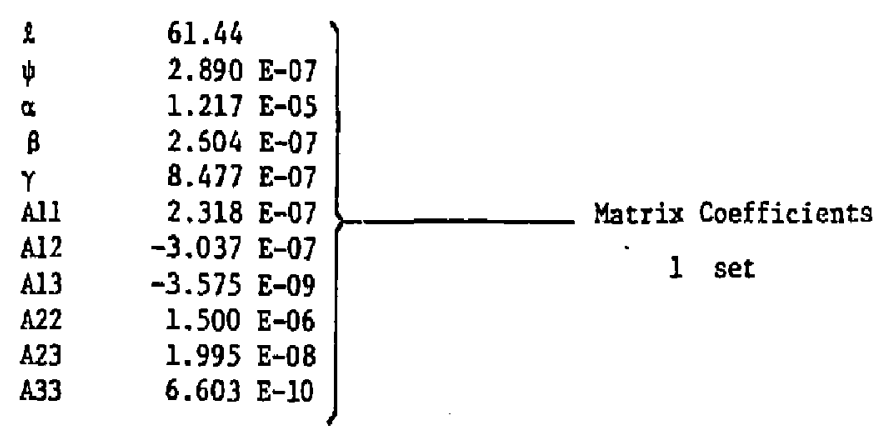

$\left.\begin{array}{lccc}\mathrm{T}_{\mathrm{SO}} & 10428 & 10428 & 10428 \\ -\mathrm{V}_{\mathrm{SO}} & -18040 & -18040 & -18040 \\ \mathrm{~V}_{\mathrm{sD}}+\mathrm{taV} & 22484 & 22484 & 22484 \\ \mathrm{~T}_{\mathrm{Sl}} & 9803 & 9803 & 9803 \\ -\mathrm{V}_{\mathrm{Sl}}+\mathrm{V}_{\mathrm{B} 1} & -16980 & 41,760 & 100500 \\ \mathrm{M}_{\mathrm{Sl}} & 45496 & 45496 & 45496\end{array}\right)$ Load Vectors

TABLE 2.5-8

ALUMTNM STRUCTURE

MATRIX COEFFICIENTS AND LOAD VECTORS

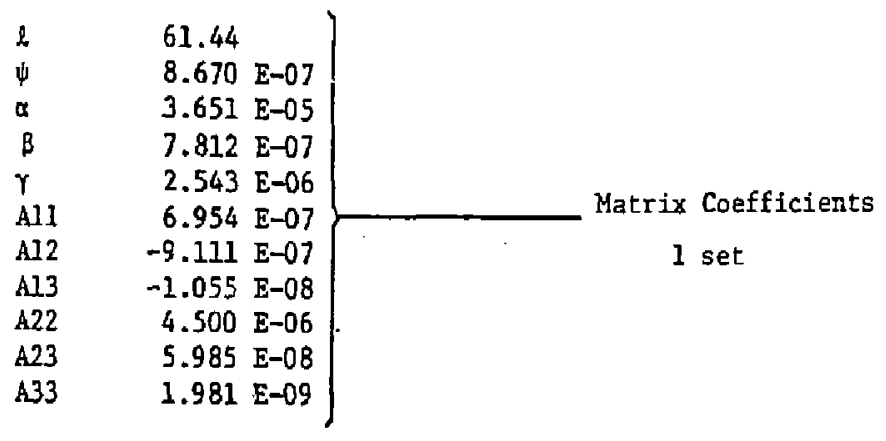

$$
\begin{array}{lc}
\mathrm{T}_{\mathrm{SO}} & 7832 \\
-\mathrm{V}_{\mathrm{SO}} & -13552 \\
\mathrm{M}_{\mathrm{SO}}+\mathrm{aV} & 15896 \\
\mathrm{~T}_{\mathrm{Sl}} & 6072 \\
-\mathrm{V}_{\mathrm{S} 1}+\mathrm{V}_{\mathrm{B} 1} & -10516 \\
\mathrm{M}_{\mathrm{S} 1} & 28160
\end{array}
$$$$
\left.\begin{array}{cc}
7832 & 7832 \\
-13552 & -13552 \\
15896 & 15896 \\
6072 & 6072 \\
29876 & 70268 \\
28160 & 28160
\end{array}\right] \quad \text { Load Vectors }
$$ 

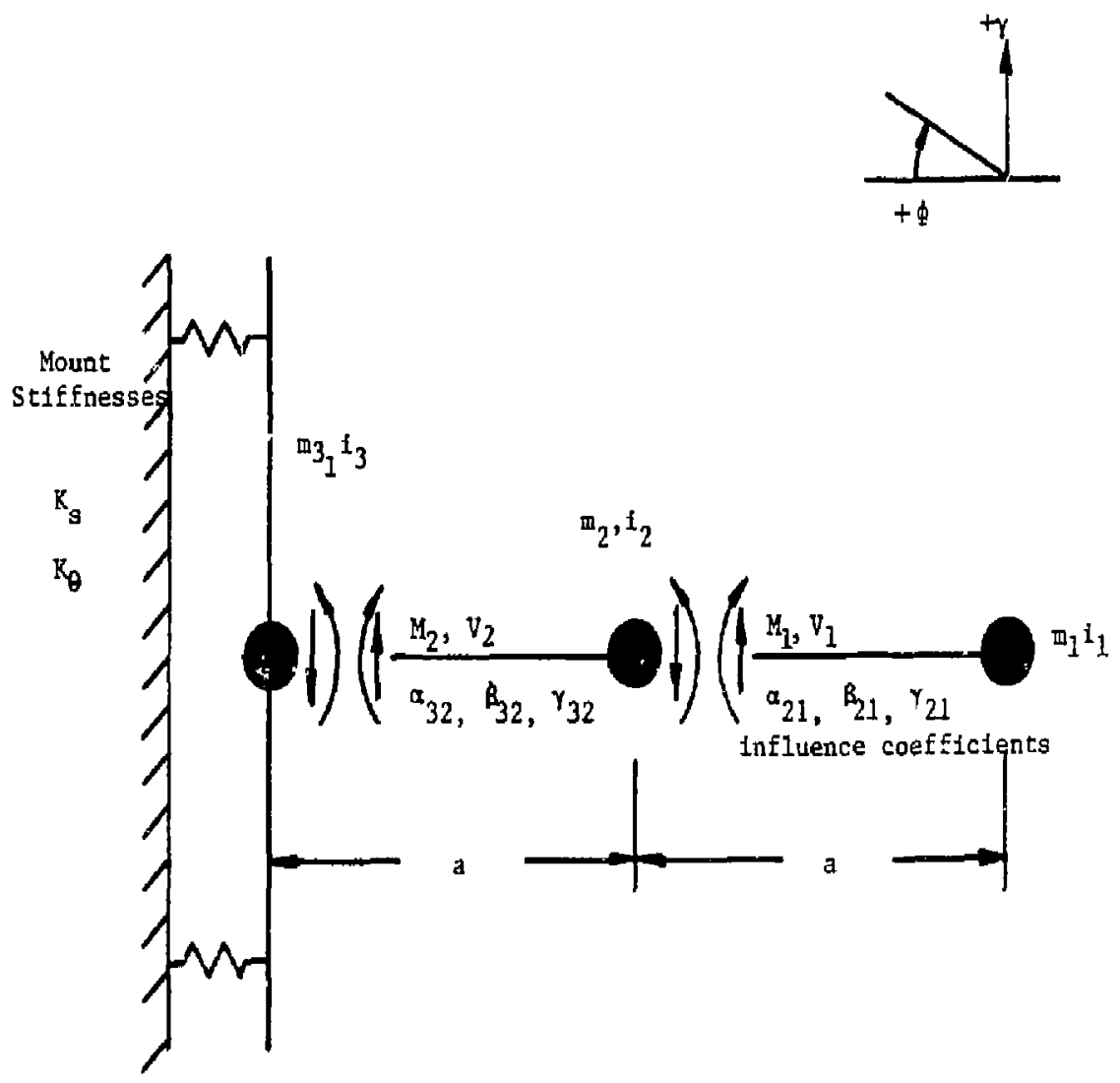

EIGUEF 2.5-5 6 DEGRSE OF FREEDOM,

PRELIMI'ARY AYIS 2 SYSTEM YODEL 

systen is much longer and much heavier. The Axis 1 system 1llustrated on DN132-165-1 does not achieve the values for "Ioundation stiffness," 1.e., $\mathbb{K}_{s}$ and $K_{\theta}$, necessary to 110 it the deflections to those prevfously estimated for the Figure 2.5-5 System.

\section{Dynamic Multipliez Estimate}

Figure 2.5-7 illustrates gee load versus time at the iocation of the Axis 2 support bearing. Note that the orientation of the load relative to the structure shifts a total of $180^{\circ}$ as the structure rotates, with a sudden $35^{\circ}$ shift when the angular acceleration goes from plus to minus whec angular velocity is at its maximum value. Such a sudden angle shift would lead to a mechentcal tmpact. This means that an acceleration schedule different from that assumed here probably will be selected. The selected schedule w1ll reflect a trade off where higher absolute values cf angular acceleration mitigate the intemediate angle shfft impact.

Flgure 2.5-8 fllustrates trlangular pulse dynamic multipliers for single degree of freedom undamped vibration systems as a function of the ratio between pulse time and response perlod. Table 2.5-9 tabli_ates dynamic multipliers for the structural cases studied here.

A real system will have many flextble modes. Also, the values tabulated in Table $2.5-9$ do not consider any variation in load orientation. Therefore, we choose to apply a dynamic mitipller of 1.75 to all static responses calculated in this preliminary study.

\section{Calculated Results}

The results plocted and tabulated here indicate that slewing system mechanical deflections can be mafutained within a range that can b. 

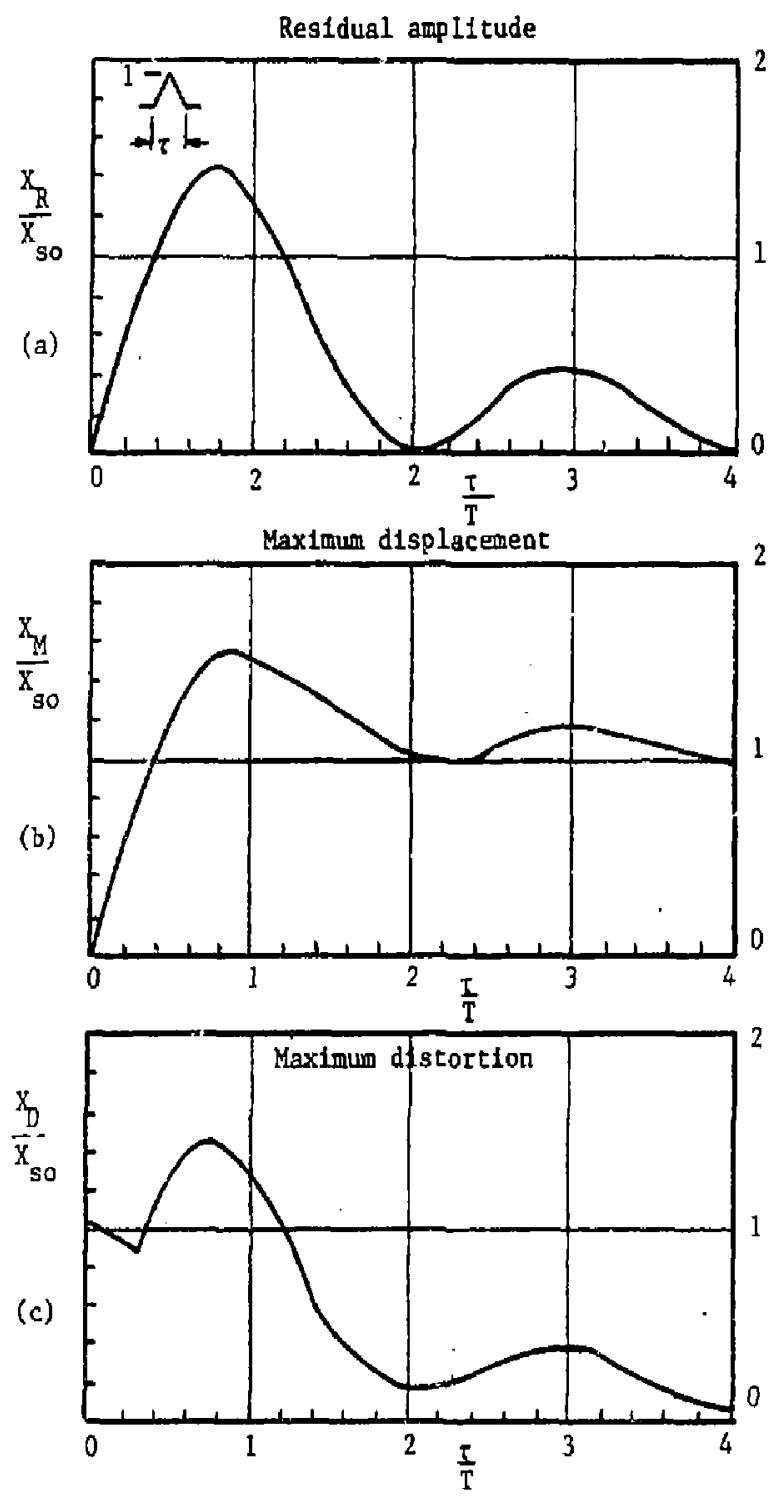

FIGURE 2.5-8 DYNAMIC MULTIPLIERS FOR TRIANGULAR PULSE VERSUS RATTO OF PULSE TIME TO NATURAL PERIOD 
TARIE 2.5-9

TABULATION OF DYNAMIC MUTTPLIER DATA

Dymanir wiltipliers

$\omega(\mathrm{Hz}) \quad \mathrm{T}(\mathrm{ms}) \quad \tau(\mathrm{ms}) \quad \tau / \mathrm{T}$

$1.29 \quad 775$

1.57

2.49

637

402

300

300

300

.387

.471

.746

.9

1.05

1.75
1111

952

571

$\begin{array}{ll}300 & .270 \\ 300 & .315 \\ 300 & .525\end{array}$

.525

1.

1.

1.3

1.25

1.2

1.25

1.

1,5

1.5

1.25

1.5

TABIE 2.5-9 TABULATION OF DYNAMIC MULTIPLIER DATA 
compensated by the Vernter Steering System. Both the Axis 1 System and AxIs 2 System can be "tuned" mechanically to limit deflection translation and rotation at the transition section exit, because the magnitudes of the tangent1ai, radial, and axlal gyrodynamic load components directly depend upon mass distribution. The mechanical designer has considerable control over the distribution of mass and structural flexibility.

Befer now to Figure 2,5-2, the schemntic diagram of the Axis 1 System response computations. $v_{1}, w_{1}$, and $\phi_{1}$ represent deflections at the transition section. Figures 2.5-9 and 2.5-10 show how variation in the Axis 1 balance weight, $W_{B}$, affects the system. Overbalancing the system can eliminate transition section translation at a cost in system weight and drive power. Flgure 2.5-11 shows a quite flat vartation for transition section angle with the transition section translation. This means that a baiance welght structure acting at the transition section could be positioned to signiflcantly reduce the absolute level of transition section rotation. Table 2.5-10 summarizes calculated mechanical loadings for the cases scudied here. The Axis 2 besring laad is consistent with the size of the selected bearings. The large Axis 1 support bearing is oversized by a factor of 2 for the highest load case. Structural stiffness, not bearing load, is the reason for the large diameter base of the Axis 1 System. The tabulated structural loads, stresses, and deflections all assume a dynamic multiplier of 1.75 .

Structural models with many mass degrees of freedom and flexible connections, tend to have higher calculated frequencles and lower stresses than the very simple two and three mass representations calculated here. 


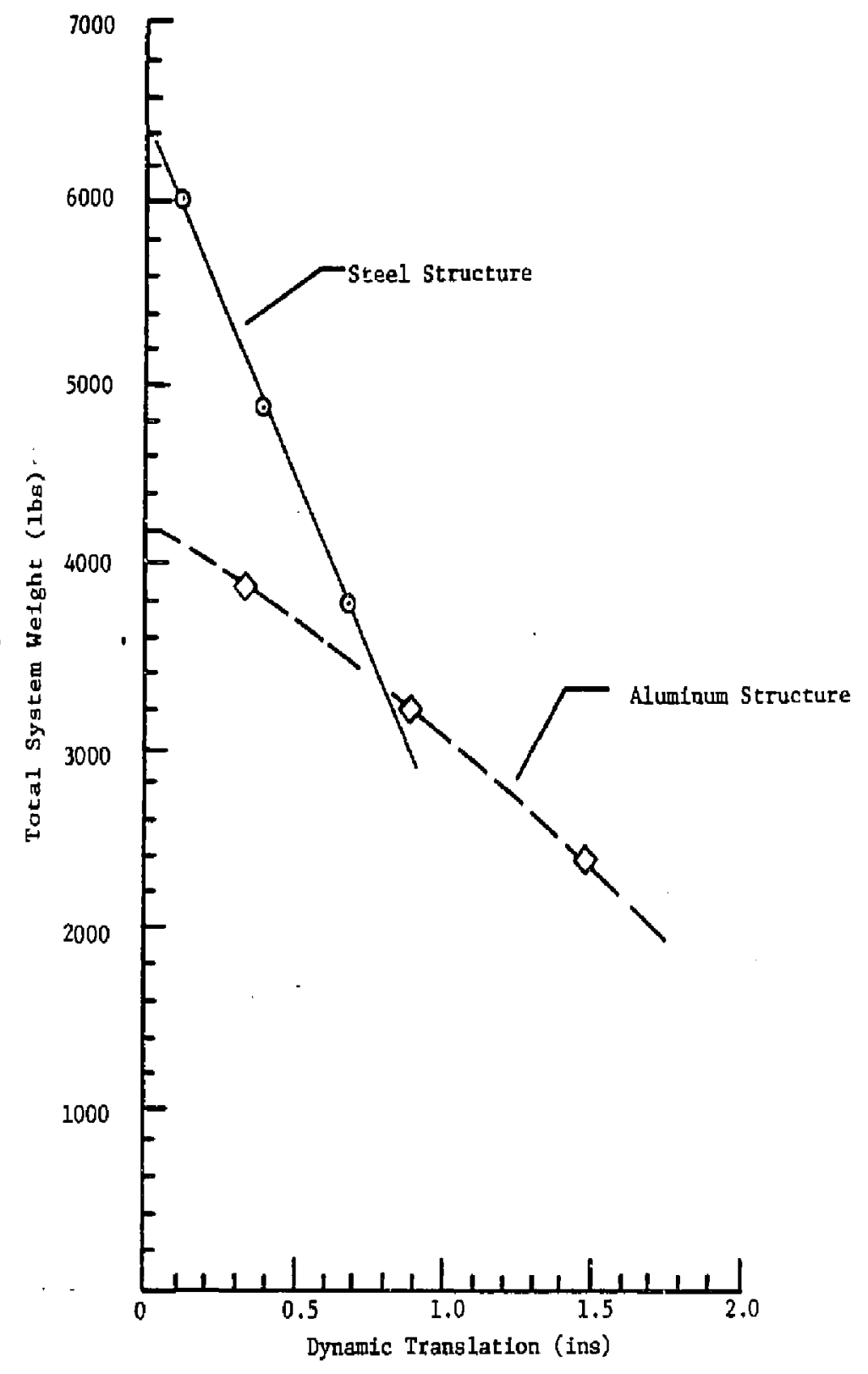

FIGURE 2.5-9 SLENING SYSTEM. TOTAL SYSTEM WEIGET VERSUS TRANSITION SECTION DYNAMIC TRANSLATION 


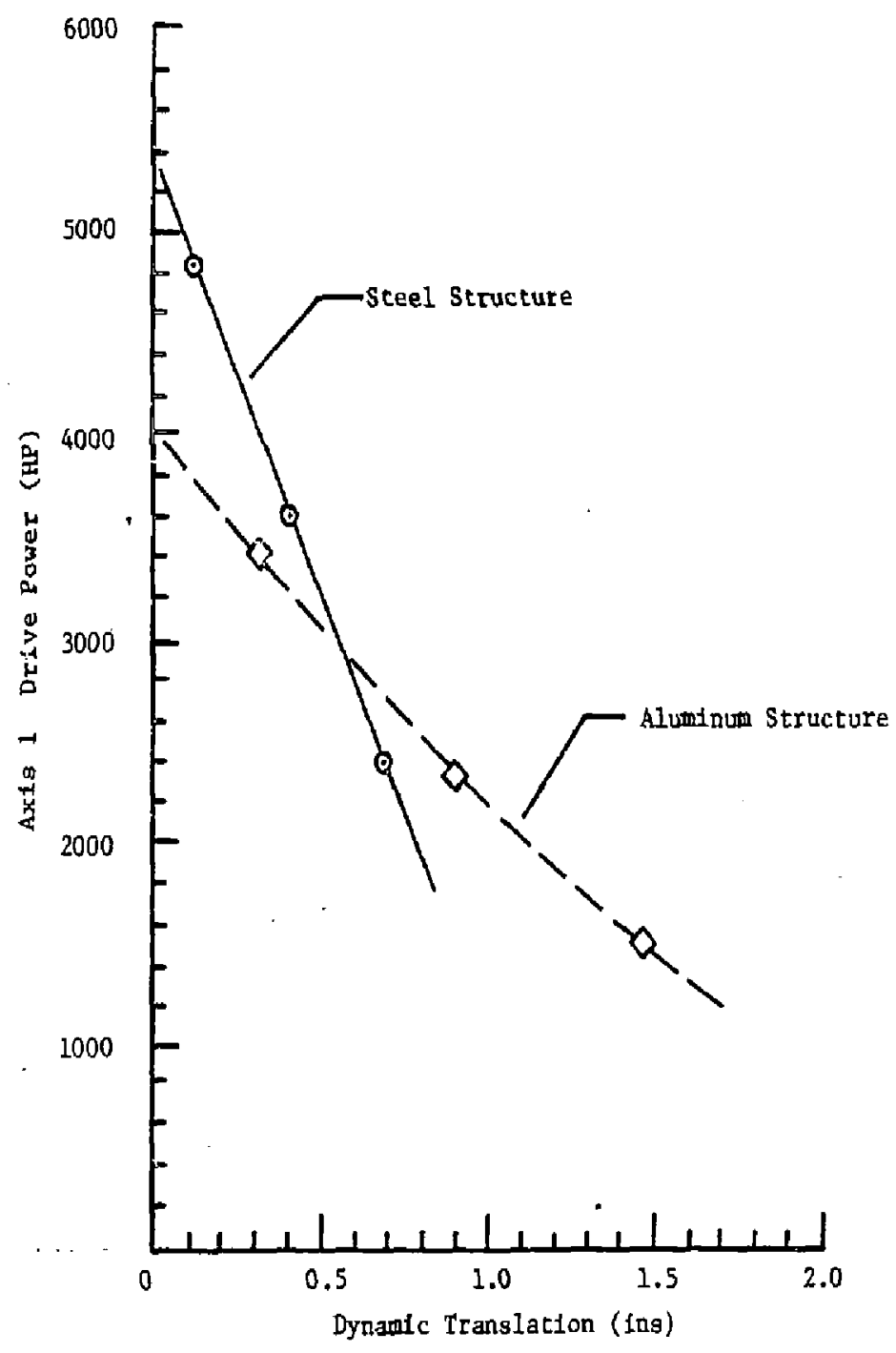

FIGURE 2.5-10 SLEWING SYSTEM. AXIS 1 DRIVE HORSEPOWER VERSUS TRANSITION SECTION DYNAMIC TRANSLATION 


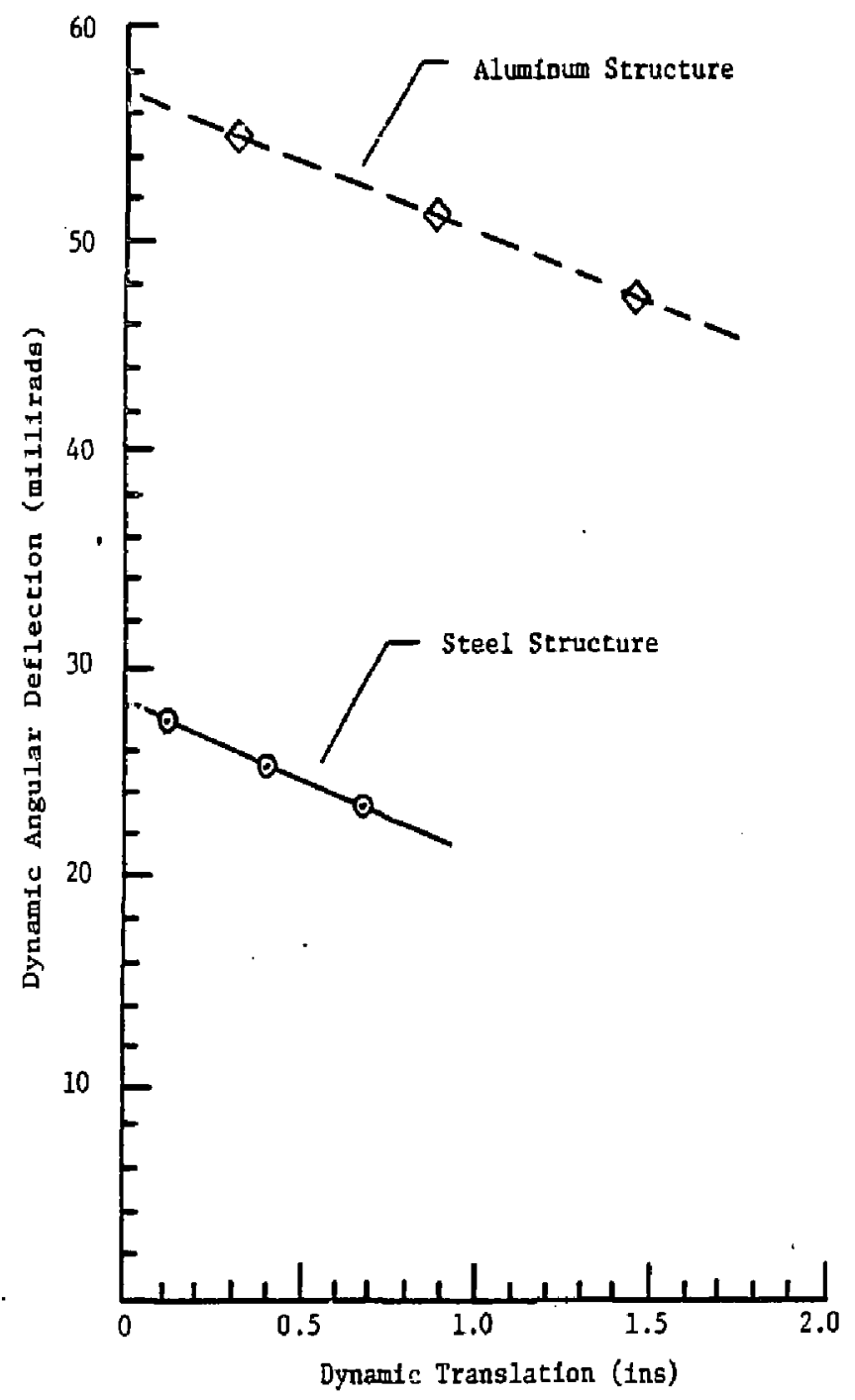

FIGURE 2.5-11 SLEWING SYSTEM. TRANSITION SECTION DYNAMIC ROTATION VERSUS TRANSITION SECTION DYNAMIC TRANSLATION 


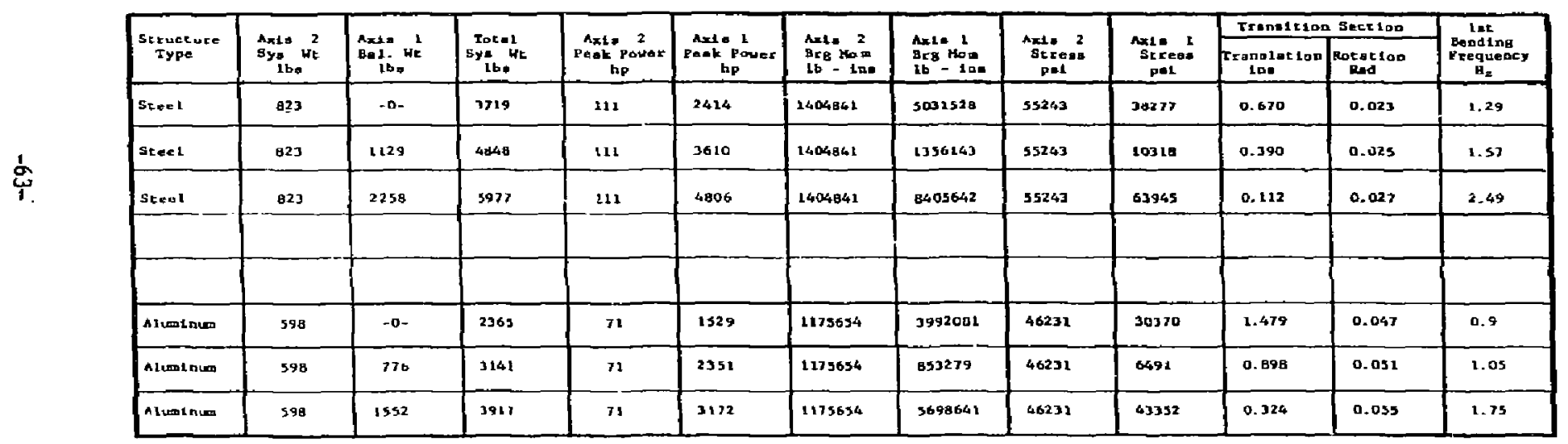


On the other hand, the pump, motors, transition section, gear drives, and other components have increased in size slnce beling initially selected. The pipe truss bending model way or may not possess enough structural weight to Ifmit deflection modes that have not yet been calculated, such as torsional modes. Clearly, these numerical tesults can only be used as a rough gulde for understanding the mechanical nature of the slewing system.

Refer now to Tables $2.5-1,2$ and Table 2.5-10. Overall stiffness varfes with structural weight and overall loading varies with the sum of structural plus cargo and balance weights. If loading geometry and time signature does not change, then additional calculated responses can be est Imated by ratfoing the values in Table 2.5-10.

$$
\Delta_{\text {new }}=\frac{\left[1+\frac{W_{C}+W_{B}}{W_{S}}\right]_{\text {new }}}{\left[1+\frac{W_{C}+W_{B}}{W_{s}}\right] \text { oid }} \times \Delta_{\text {old }}
$$

Where $W_{C}, W_{B}$, and $W_{B}$ are cargo, balance, and structure weights from Tables 2,5-1 and 2. In the case of 2,5-2 for the Axts 1 System, the items for "tubes and lower structure" should be jeft out of the above calculation.

The additional power requirement w11 also vary with the above welght ratio equation.

CONCLUSIONS: The structural veight and stiffness will determine the vibrational frequencies and, dependent on the slewing rates, these will also determine the dynamic deflection.

Adequate structural rigldity can be provided to limit the deflection of structure to a tolerable amount as determined by the following two primcipal limiting criteria for tolerable deflection: 
(1) Maximum afming error that can be compensated for by the vernier steering ragnet and

(2) maximum structural misaligntment of the magnetic components of the achromat.

Since the achromat can accomodate a significant (1\%)

varfation in energy, it will also be able to accommodate a limited (1\%) structural deflection.

Increasing the stiffoess of the structure requires an increase in Its weight and drive power. Since all of these increases very rapidiy with Increasing energy, the weight and power will become so large that it will be very difficult to achleve useful slewing rates for beam energy in excess of $1000 \mathrm{MeV}$. 


\subsection{Radiacton Effects}

The main radtation effects are those associated with collistons of the high energy electrons with the wall, exit foll and gas molecules. There will also be some synchrotron radiation due to bending the beam in a magnetic fleld. The sypchrotron radiation is given by the following equation:

$$
U=8.85 \times 10^{-32} \frac{(W / e)^{4}}{r}
$$

Where $U=$ ens rgy loss ev/rev

W/e = energy of electron in ev

$\mathbf{r}=$ bending radius in meters

For a $500 \mathrm{MeV}$ electron beam being bent through a 60 degree angle with a bend radius of curvature of I meter, the energy loss 1s only $922 \mathrm{ev}$,

This is a very small fraction of the total beam energy. For the very high beam currents contemplated for the electron beam director, the tatal cadtation from the source may amount to ouly a few foules for a =mult1pulse burst. The peak radiation would be in the far ultraviolet region and would be mostly tangential to the beam path.

The radiation due to gas scattering in the vacuum chamber is expected to be extremely small if a high vacum is maintained in the beam tube. At a pressure of $10^{-3}$ torr, the beam loss due to gas scattering will be insignificant.

Radiation due to collisons with the wall of the vacuum chamber will be extrenely bigh if the beam is not well behaved in its transmission. through the beam director. Collisions with the wall by even a small fraction of the beam must be avolded because an excessive amount of tadiation would be produced. It will be necessary to "trim" the beam upstream of the beam 
director to limit its size (in phase space) to assu:ee that it does not spread out far enough to scrape the walls. The lintting beam radius is 5 mm.

The radiation due to the beam passing through the exit foll will be unavoldably large. It w11 conslst of high energy $x$-rays whth a very strong forward distribution due to momentum conservation in the electronelectron impacts. The $x$-rays will have a continuous spectrum characteristic of bremsstrahlung whth a maximum energy equal to the energy of the electron. The half-angle in the forward direction which lacludes all bur $1 / e$ of the intensicy is given by

$$
\theta_{\mathrm{i}}=.65\left(\frac{\mathrm{WO}}{\mathrm{H}}\right) \text { la }\left(\frac{\mathrm{W}}{\mathrm{Wo}}\right)
$$

Where Wo $=$ rest mass of electron

$$
\text { W } \Rightarrow \text { energy of beam }
$$

For a $500 \mathrm{MeV}$ electron beam, the half angle is about one quarter. of a degree, for a low emittance beam. High emittance will increase the spread.

The total amount of radlation due to passing the beam through the exit foj.l will depend upon the foil thickness and its density. A very thin foll of low density will minimize the radiation effects. For a carbon or beryllium foll one millimeter thick, the beam loss is expected to be less than $.5 \%$ of the total beam energy but 1t does represent a large amount of radiation.*

Figure 2.6-1 shows the cadiation loss for the beam passing through an exit foil of elther berylltum or carbon and the loss in air. This figure shows the loss for collisions and for radiation. Clearly, tie radiation loss Increases with energy. The loss due to collision is a very weak function of energy.

*The essentlal point is that strong forward distribution assures that the bulk of the radiation from the foll does not intersect the coils of the vernier steering magnet, and that this must be confirmed in any actual design. 


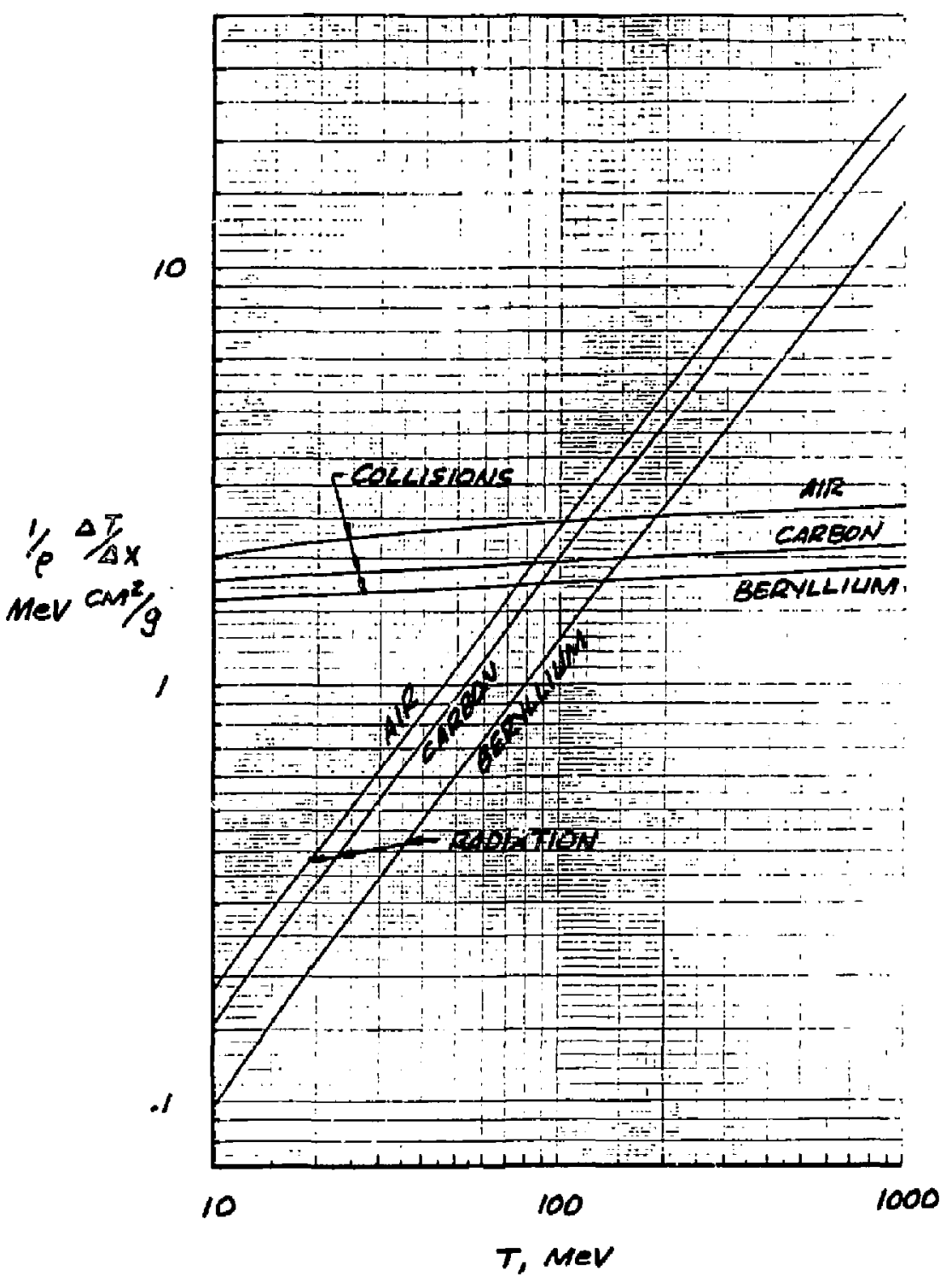

FIGURE 2.6-1 BEAM LOSS DUE TO RADIATION AND COLLISIONS FOR BERYLLIUM AND CARTION FOILS AND FOR AIR (Reference: Berger \& Seltzer) 


\subsection{Size, Weight and Cost Estimate}

The sfze, veight and cost of a $500 \mathrm{MeV}$ electro beam director

were calculated. Ali these values are dependent upon the seam energy. A range of energies from 10 to $1000 \mathrm{MeV}$ were considered for the conceptual design and the concepts presented are considered to be applicable to any energy within that range. Some simple scaling law would be desirable. For example, it would be nice to be able to say that doubling the energy would double the length of the bending systems and that thits, fn turn, would cause some easily extrapolated increase in weight and cost. Within narrow limits, sope sort of extrapolation based on energy ratlos is reasonable because the same permanent magnet material would be used for a limited range of energies. For beams with very high energies, a rare-earth-cobalt with a high residual induction would be used. For low energies, orlented ferrites would probably be selected and, for Intermedlai:e energi zs, rare-earth-cobalts of intermediate residual induction may ie selected.

For a given permanent magnet materlal, an increase in energy will require a proportional increase in length and weight of the bending system. The weight of the support system will increase approximately with the cube of the energy as it must not only increase in length but also in the transverse directions. The moments of inertia will not oniy increase with weight but also with the iquare of the of fset dimension. The moment of inertia has a strong influance upon the design of the mounting and slewing system. Frow the increase in dimensions and weight associated with increasing beam energy, it appears that the toment of Inertia wili increase by at least the 4 th power of the energy ratio.* Thus, the required drive power for a given slewing rate wiil also increase by at least the 4 th power. the exponent probably approaches the 5 th power at high energy. 
The values given below for the $500 \mathrm{MeV}$ beam director are based on an unproven conceptual design. As stated aboye, within limits, these values may be scaled up or down for different beam energies.

The results of the size, weight and cost analysis are sumarized in Table 2.7-1. The costs are the unit costs in production and do not include development costs.

TABLE 2.7-1 SUMMARY OF SIZE, WEIGHT AND COST

\begin{tabular}{|c|c|c|c|}
\hline ITEM & $\begin{array}{c}\text { Dlinension } \\
\text { Meters }\end{array}$ & $\begin{array}{l}\text { Weight } \\
\mathrm{Kg} \\
\end{array}$ & $\begin{array}{c}\text { Cost } \\
\mathrm{K} \$ \\
\end{array}$ \\
\hline Beam Bending System & 3.14 length & 227 & 152 \\
\hline Support Structure & $3.14 \times 2.9410 \mathrm{E}$ & $2700^{\star}$ & 154 \\
\hline Drive System & - & $4000^{\star \star}$ & 501 \\
\hline Vacuum System & - & - & 81 \\
\hline Vacuum/Air Transition & .25 & 25 & 105 \\
\hline Vernier Steering & .5 & 50 & 40 \\
\hline Sensors and Control & - & - & 100 \\
\hline Total & $3.89 \approx 2.9$ Wide & 7000 & $\$ 1133$ \\
\hline
\end{tabular}

* Weight of rotating structures (5500 lbs) Includes upper drive motor.

** Weight of stationary portion of the drive system includes lower drive motor, hydraulic pups, accumulatiors and foundation structure (8800 1bs). 


\subsection{RECOMHENDED RESEARCH AND DEVELOPNENT}

The conceptial design of an electron beam director contains elements which require developmental effart. To support the development w1ll require research to provide basic data on behaviour of material and interactions of the beam. There is need for a design and development effort assoctated with many elements of the system, but some elements are more critical than others. Singie quadrapoles and dipoles using permanent magnets in an ironless configuration have been designed and bullt. A long string of such elements as shown in the conceptual design has not yet been bullt and tested.

Air-to-Vacuum Transition devices as shown in the conceptual design utilize known cechnologies but these have not been proven in the specific application shown in the conceptual design.

Procedure for analyzing the dynamic response of structures to various types of loads are well known, but spectfic structures to support a beam director have not been sufftciently well defined nor has a complete slewing system been designed. The brief examination of a support and a slewing system concept indicates that a great amount of destgp and optjmization will be required in order to achleve a practical design. A light weight structure with high rigidity is required if high slewing rates are to be achieved.

The steering magnets for the Vemler Steering System are of a conventional design, but the tracking and concrol system needed to assure that the required accuracy and vibration compensation is achleved is yet to be developed. An additional problem associated with the design of the steering magnet is the potentfally high radiation which may occur fust downstream of the Vacuum-to-Air Transition Secton. The radiation problem is 
treated conceptually by simply proylding enough iferture to permit the radiation which is directed strongly forward, to pass through without great Interaction with the material of the steering magnet. Some critical experfments of the beam/air Interaction will be requifed to verify this approach.

Probably, the most critfcal ftems in the conceptual design are the Vacuum-to-Air Transition Section and the permanent magnet bending system. Design and development work on these two items should be started soon. Both systems can be tested using the electron beam from ATA (Advanced Test Accelerator) at Lawrence Livermore Natfonal Laboratory.

The rotating foll approach to the Vacuum-to-Alr Iransition could be butlt, tested and tefined using presently awallable tecbnologies. The development of such a device would permit the runalng of many critical experiments dealing with beam/air interaction. The development of this rotary foll device will require the developtent of a well-balanced rotor, a suitable vacum pumping system and a suitable bearing system. In the final configuration, the gyrodynamic behaviour of the device must be suitably accounted for, but, for the intrlal developmental device, the gyroscopic loading of the disk and bearings can be avoided by simply testing in a static configuration.

A permanent magnet bending and focussing system should be butlt for tescing at ATA. This should be degigned so that it can be rotated to direct the beam in various directions. A continuous rotation may be desirable but not absolutely necessary. Discrete steps should be all right. The main thing to explore in this design and developmenc is the characteristics of the permanent magnet hending system. The problems of accuracy of 
mechanical and magnetic alfgnment needs study, How rellable are the magnetic property projections? What are unique fabrication problems? What sort of optical aberations will occur? What are the conseguences of vibration and misalignment? Buflding and testing a system will provide answers to these questions.

For the beam energy at ATA (50 MeV), a bending and focussing system using an oriented ferrite guch as Ferrimag 7B should be bullt. This material will give a lower field than that obtalnable with rare-earth-cobalt. The lower fleld is destrable for a $50 \mathrm{MeV}$ electron beam because it gives a greater length of the system. 


\subsection{CONCLUSIONS}

In the engineering effort on the conceptual design of an electron beam director, a variety of components and concepts were examined, and found to be promising. There were no technical reasans for belleving that a suitable design could not be devised for handling high intenslty pulsed electron beams in the 10 to $1000 \mathrm{Me}^{\text {Ir }}$ energy range. Of course, there are problems which wust be solved, and there are limits imposed by materlal propecties which must be recognized.

Achieving high slewing rates will be difficult but not impossible. Spectal design efforts will be required to produce a sufficlently li, ht weighl support structure having adequate rigidity.

The most eritical elements of the design appear to be the Vacum-tnAir Transition, the permanent magnet bending and focussing system, and the support structure. The design of the first two of these items has a strong influence on the design of the support structure, because the weights and alignment requirements of these components determine the key design criteria for the rest of the system.

Presently, available permanent magnet materials appear to have suitable magnetic properties to satisfy the design requirements for bending and focussing of the beam. Whether these materials have the required physical strength and uniformity is not certain, but there are no fundamental reasons why good quality control procedures cannot assure adequate unfformity nor why existing physical propertles cannot be utilized by careful design of the magnet support and containment assembly. 
The aperture of the beam tubes can be made suffictently large to insure that the beam loss by collistons with the wall will be insignificant and that adequate vacuum pumping will be possible to prevent excessive bean loss due to gas scattering. Th1s will be necessary to minimize radiation.

The vacuum totary polnts will have some leakage but, by proper design with at Ieast two stages of pumping, the leakage rate can be wade tolexably low. The joints can be made sufficiently stiong to provide adequate rigidicy and alignment.

Radiation effects can be minimized by good desigri and sultable s.ielding. The radiation from the beam passing through the Vacuum-to-Air Transition Section will be unavoldably large but, at this point, the electron beam/air inceraction may be so great that other radtacton effects may seen insignificant.

The calculated size of the total structure does not appear excessively large although the weight of seven tons does s zem high for a $500 \mathrm{MeV}$ beam. It is belleved that this welght may be significantly reduced after design optimization for a specific set of beam requirements. It seems premature to begin detalled design unt1l a specific value of beam energy has been selected.

Using a value of $50 \mathrm{MeV}$ would permit the use of a much. lighter structure than would be needed for a $500 \mathrm{MeV}$ beam because the weight is expected to Increase by about the third power of energy. Thus, a factor : 10 increase in energy would require about 1000 fold increase in the weight of the support structure.

The rotary vacuum jolnts were inltlally thought to present serious design problew, but do not now appear to be excessively difficult. It appears that lubricated vacuum seals with intermediate pump-out ports are viable. 
5.0 APPENDIX

DESIGN STUDY SKETCHES

DN132-165-1A

DN132-165-1B 
CHANGES MADE ON

"CONCEPTUAL DESIGN OF AN * ECTRON BEAM DEFLECTOR"

BY LBL PRIOR TO DISTRIBUTION

1. Added to bottom of Title page: Lawrence Berkeley Laboratory Contract No. 4522410.

2. Page ii: Removed "5,0 Appendix"

3. Page 7: Replaced bracket on equations.

Changed equation to: $\theta=\frac{\pi}{2}-\sin ^{-1}\left(1-\sin ^{2} y\left(1+\cos \alpha_{2}\right)\right)$

Changed equation to: $\phi=a_{1}+\tan ^{-1}\left(\frac{2}{\sqrt{3}} \frac{(\sin \alpha)}{\cos q_{2}+1}\right)$

4. Page 9: Replaced brackets on equation

5. Page 14: Replaced parenthesis on equations

6. Page 23: Changed $\mathrm{Hr}_{d}$ with $\mathrm{Wt}_{d}$ in column 7

7. Page 37: Removed reference to Appendix--replacad with "(not included)" two times

8. Page 38: Added "*" after sketch no, and included footnote at bottom

9. Page 46: Added "*" after sketch no, and included footnois at bottom

10. Page 50: Added "*" after sketch no, and included footnote at bottom

11. Page 65: Fourth line from bottom, changed "increases" to "increase"

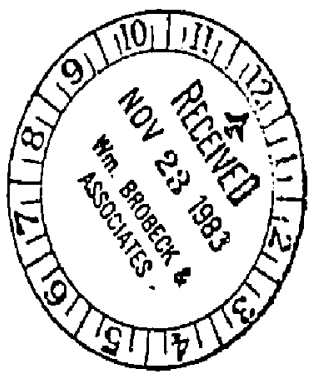


APPENDIX H. BEAM DIRECTOR COMPONENTS FOR ATA TESTS (FINAL REPORT)

H-1 
BEAM DIRECTOR COMPONENTS

FOR

ATA TESTS

\author{
Prepared for \\ LAWRENCE LIVERMORE NATIONAL LABORATORY \\ LIVERMORE, CALIFORNIA
}

\author{
Prepared by \\ BROBECK CORPORATION \\ 1235 Tenth Street \\ Berkeley, California 94710 \\ (415) 524-8664
}

DECEMBER 1985

Report No. 4500-300/301-R1

= This work was done under Subcontract No, 6551805 with Lawrence Livermore National Laboratory, Livermore, California.

This work was sponsored by PMj̃-405 Naval Sea Systems Comanand, Washington, D.C. 20362, under Contract No. W-7405-ENG-48 with the United States Government, represented by the Department of Energy. 
BEAM DIRECTOR COMPONENTS

FOR

ATA TESTS

Prepared for

LAWRENCE LIVERMORE NATIONAL LABORATORY

LIVERMORE, CALIFORNIA

\author{
Prepared by \\ BROBECK CORPORATION \\ 1235 Tenth Street \\ Berkeley, California 94710 \\ (415) $524-8664$
}

DECEKBER 1985

Report 10. 4500-300/301-R1

This work was done under Subcontract No. 6551805 with Lawrence Livermore National Laboratory, Livemore, Californta.

This work was sponsored by PMS-405 Naval Sea Systems Command, Washington, D.C. 20362, under Contract No. W-7405-ENG-48 with the United States Government, represented by the Department of Energy. 
APPROVALS

Issue Contents

Prepared by Approved hy

Original $v+35$ pgs .

Francjs C. Younger

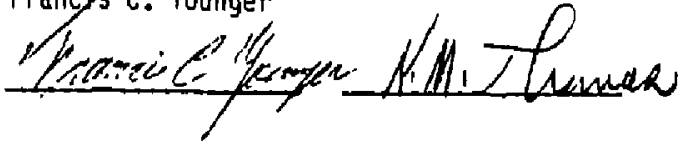




\section{TABLE OF CONTENTS}

\begin{tabular}{|c|c|}
\hline BEAM OIRECTOR COMPONENTS FOR ATA TESTS & 1 \\
\hline REQUIREMENTS & 3 \\
\hline DESIGN DRAWING & 11 \\
\hline MEASUREMENT ANO SORTING OF P.M. SEGMENTS & 12 \\
\hline MAGNETIC MEASUREHENT ON ASSEMBLIES & 26 \\
\hline ARRANGEMENT OF ACHROMAT ELEMENTS & 29 \\
\hline TESTS AT BROBECK CORPORATION & 30 \\
\hline RECOMMENOATIONS FOR POHER SUPPLIES & 31. \\
\hline RECOHMENDED TEST PROGRAM & 33 \\
\hline ACXNOWLEDGEMENTS & 35 \\
\hline APPENDIX & 36 \\
\hline Achromat Bend Magnet Drawing list & 37 \\
\hline Vernier Steering Magnet Drawing List & 38 \\
\hline
\end{tabular}

AGE 


\section{LIST OF FIGURES}

PAFF,

1 Magnetic Lattice Showing the Arrangenent of Quadrupoles and Bending Magnets (Dipoles)

2 Permanent Magnet Dipole

3 Pemanent Magnet Quadrupole 


\section{LIST OF TABLES}

PACE

1 Vernier Steering Magnet Characteristics

2 Achromat Characteristics

3 Beam Path Through Achromat with Shown Elements

4 Magnetic Measurements Engineering

5 Segment Series 100 -- Quadrupole \#S1 -- "S2

6 Segment Series 200 -. Quadrupole \#3(B-D) - $5(2-D)$

7 Segment Series 200 -- Quadrupale \#7(7-0) - \$9(6-D)

8 Segment Series 300 -. Quadrupole $\$ 2(4-F)$ - $4(5-F)$

9 Segment Series $300--$ quadrupole $\# 6(3-F)$ - $\$ 8(1-F)$

10 Segmant Series 400 -- Dipole $\# B 1$ - $\# B 2$

11 Segment Series 400 -- Dipole \#B3 - \#B4

12 Segment Series 400 -- Dipole \#B5 - \#B6

13 Segment Series 400 -. Dipole $\$ B 7$ - \#B8

14 Segment Series 400 -- Dipole $\$ B 9$

15 Quadrupole Assemblies Measurements Summarization

16 Brobeck Permanent Magnet Dipoles

17 Power Supplies and Expected Fields for Beam Director 32 
A system for directing a high-energy electron beam had been studied earlier and a conceptual design had been developed. Several key components of this design were identified and targeted for verffication tests using the high intensity electron beam from ATA. This report covers two of these components--the achromat and the vernier steering magnet wich were built for testing at ATA.

The achromat is a major beam transport element of the beam director. It is designed to provide a 30 degree achromatic bend of a $45 \mathrm{MeV}$ electron beam using a combination of permanent magnet dipoles and quadrupoles. The dipoles provide the bean bending and the quadrupoles provide focusing to assure achromaticity to a first order. Since higher order chromatic aberration may be present, a system of trim coils is provided to pemit adjustments in the quadrupole gradients to compensate for beam energy variations. The permanent magnet elements are made from a relatively weak oriented grain ferrite magnetic material. As a beam bending and focusing device, its most unique feature is its light weight, which is required to permit the rapid slew rates needed for a bean director. Testing at ATA is expected to show the extent to which the achromat is sensitive to the space charge associated with high intensity beams and to pemit an evaluation of the vacuum requirements for successful steering and focusing a high intensity beam.

The vernier steering magnet is an electromagnetic device for producing a finely controlled angle of bend for the electron beam. The device has two sets of coits for bending the beam. One set bends the beam in the horizontal 
plane and the other bends the beam in the vertical plane. By suitably energizing both sets of coils, the direction and magnitude of the bend can be controlied. The device is designed to produce a maximum bend of two degrees in any direction. The peak magnetic field required ir fairly low so that iron is not required to provide a flux return path. A major requirement of the design is that it be light weight so that high slew rates may be achieved. For the tests at ATA, the vernier steering magnet is installed in a vacuum tank so that the environment can be controlled. The tests at ATA should provide answers to critical questions on the suitability of this type of vernier steering element operating with a high space charge beam and/or with ionized gases th the magnetic aperture. 


\section{REQUIREMENTS}

The vernier stcering magnet is designed to produce a 2 degree hend of a 45 MeV electron beam using two orthogonal sets of coils which can he separately energized to control the direction and magnitude of the bend angle. Table 1 lists the target spectfications and shows as-built values based on magnetic measurements. As may be seen, the required current is somewhat lower than targeted becalse the effective magnetic length is longer than initially projected. The coil resistance is slightly higher: consequently, the required voltage is only slightly higher than the design objective.

The achromat is designed to produce a 30 degree bend of a $45 \mathrm{MeV}$ electron beam using permanent magnet dipoles and quadrupoles in a lattice structure designed to give a 360 degree phase advance. The target characteristics for the design are listed in Table 2. The arrangement of the dipoles and quadrupoles are shown in Figure 1 and the arrangement of permanent magnet elements required to produce dipole and quadrupole fields are shown in Figures 2 and 3 . The focusing characteristics are shown in Table 3.

The magnetic strength of the permanent magnet sectors varies slight?y from element to element. Also, the alignment of the magnetic easy axis may vary slightly from the exact location desired. To account for the variations in alignment and magnetic strength and to minimize any adverse effect these may have upon the achromat, each segment was separately measured for magnetic moment and orientation of magnetic axis by the Magnetic Measurement Group at Lawrence Berkeley Laboratory (LBL). Using these measurements, the elements were sorted prior to the assembiy in dipoles and quadrupoles. 
TABLE 1. VERNIER STEERING MAGNE İ CHARACTERISTICS

\begin{tabular}{|c|c|c|}
\hline COIL LENGTH & $40 \mathrm{~cm}$ & \\
\hline MAXIMUA FIELD & 147 gauss $\pm .5 \%$ & \\
\hline MAXIMUM BENO & \pm 2 degrees, $\pm 5 \%$ & \\
\hline DIRECTION & $x$ or $y$ & \\
\hline PROJECTED VALUE & $x=$ COILS & $y-$ Colis \\
\hline COIL RADIUS - inches & 1.5 & 1.25 \\
\hline NUMBER OF COIL TURNS & 78 & 78 \\
\hline COIL RESISTANCE - ohm & .766 & .736 \\
\hline CURRENT FOR $2^{\circ}$ BEND - amp & 22.8 & 19.0 \\
\hline VOLTAGE - volts & 17.5 & 14.0 \\
\hline INDUCTANCE $=\pi H$ & 1.26 & 1.05 \\
\hline MATER IAL & ALUM & AlliM \\
\hline WE IFHT - pounds & 1.5 & 1.5 \\
\hline \multicolumn{3}{|l|}{ AS BUILT VALUES FOR $45 \mathrm{MeV}$} \\
\hline CURRENT FOR $2^{\circ}$ BEND - amp & 18.3 & 15.25 \\
\hline RESIST & 1.00 & .95 \\
\hline VOL.TAGE & 18.3 & 14.5 \\
\hline EFFECTIVE MAGNETIC LENGTH - cm & 46 & 45 \\
\hline
\end{tabular}


TABBLE 2

ACHROMAT CHARACTFRISTICS

FNERGY

LATTICE

MAGNET NEVICES

BEAM TLIBE APERTURE $\quad 2.84 \mathrm{cM}$

MAGNET APERTIIRE

$3.24 \mathrm{~cm}$

OIIADRUPOLF CHARACTERISTICS (16 OUADS)

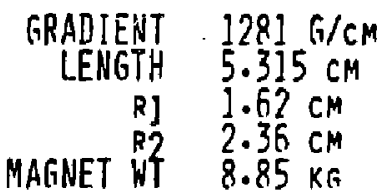

DIPOLE CHARACTERISTICS

\section{(8 DIPOLE)}

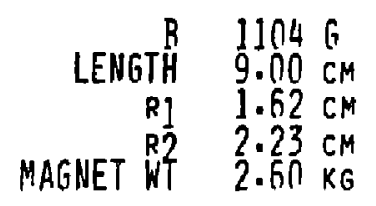

PERMANENT MAGNET MATERIAL

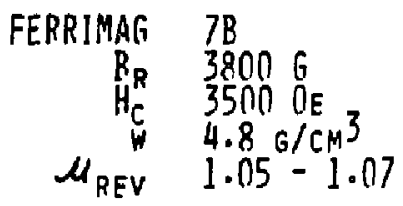

TRIM COIL CHARACTERISTICS

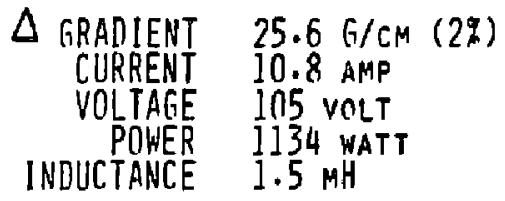

COOLING 
FIGIIRE 2. PERMAMENT MAGMET DIPOLE

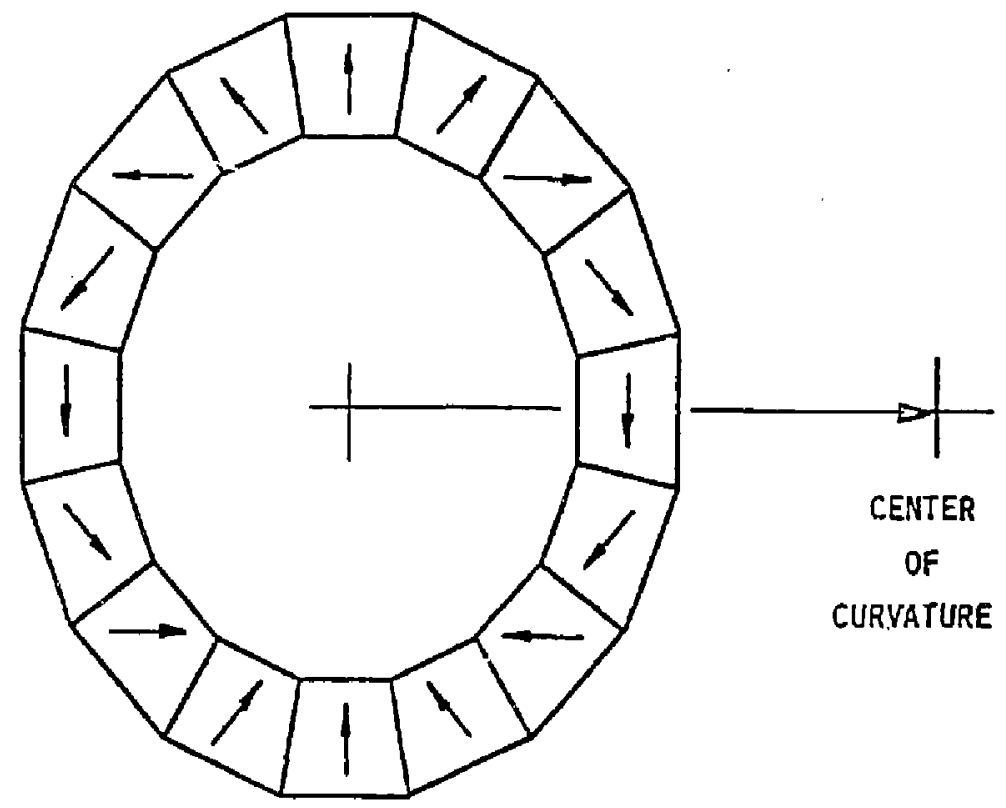

I6 PERMAMENT Maghet SEgieNTS

ARROWS SHOW DIRECTION OF MAGNETIZATION 
FIGURE 3. PERMANENT MAGNET QUADRUPOLE

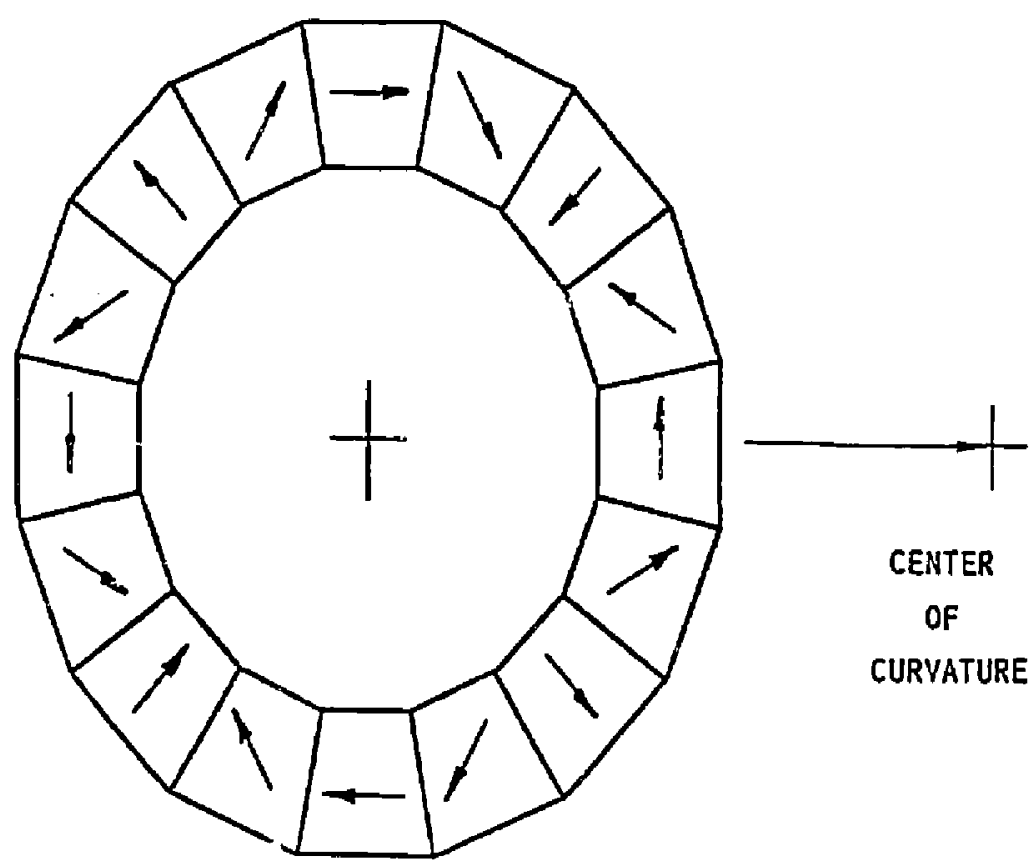

16 PERMANENT MAgNET SEgMENTS

ARROHS SHOW DIRECTION OF MAGNETIZATION 


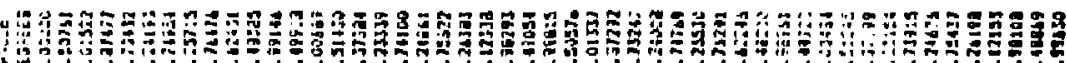

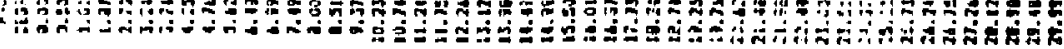

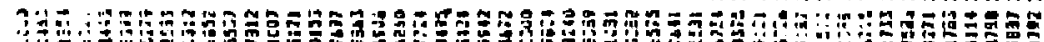

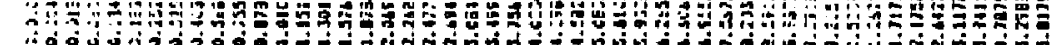

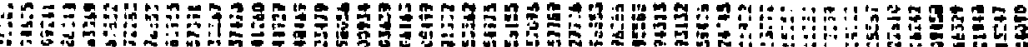
4 in

旁

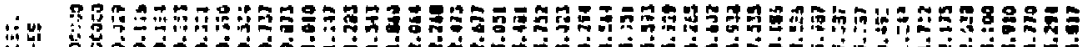

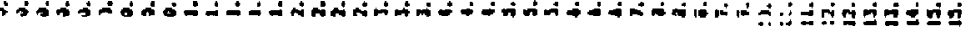

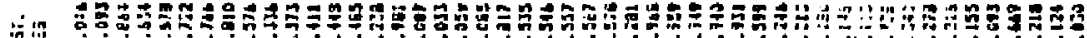

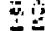

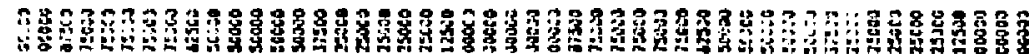

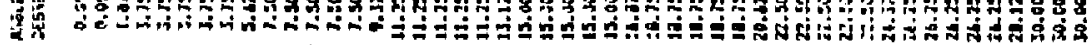

4 -

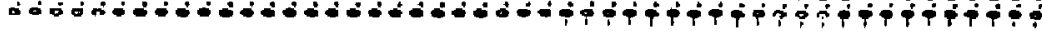

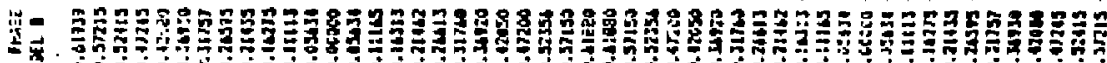

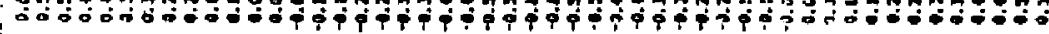

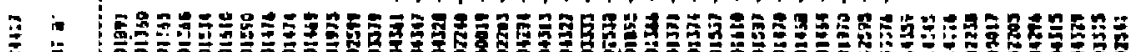

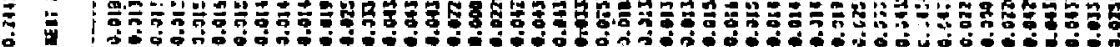

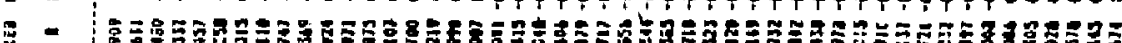

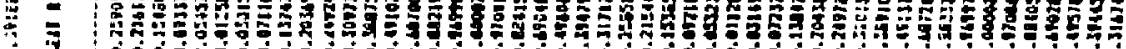

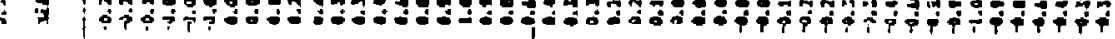

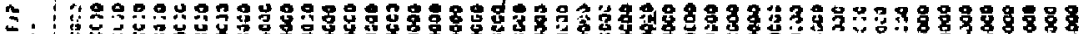

(1)

- 는

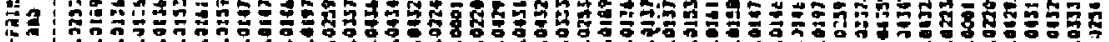

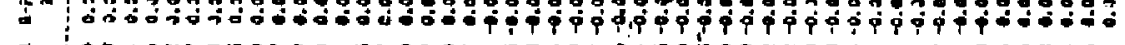

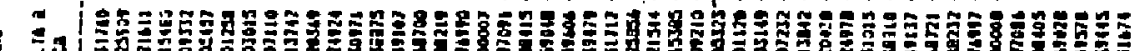

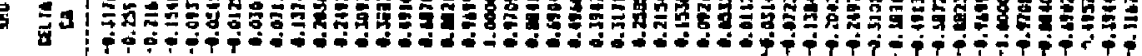

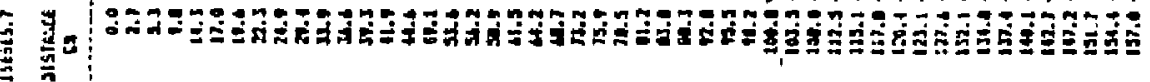

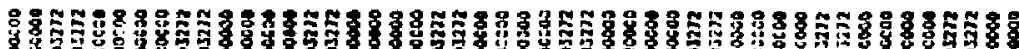

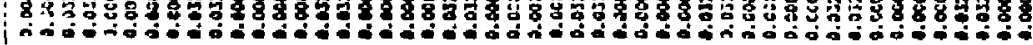

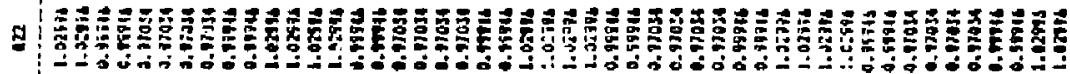

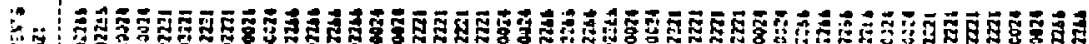

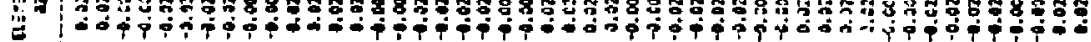

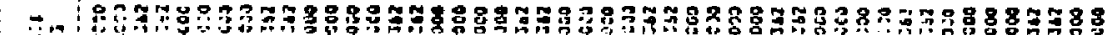

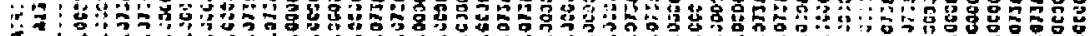

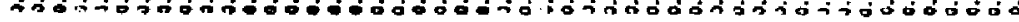

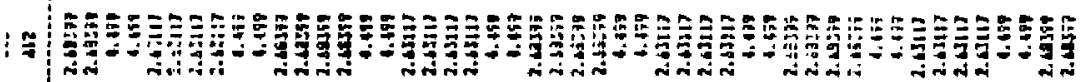

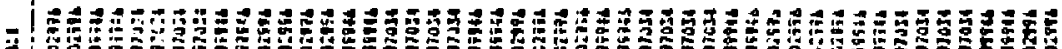

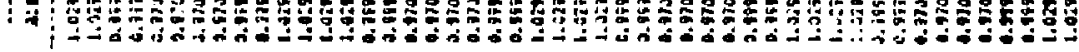
A

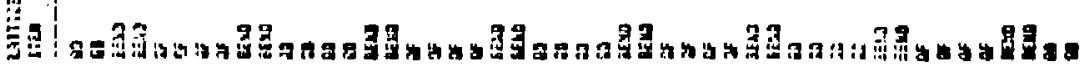


The assembled dipoles and quadrupoles were also measured at LBL. The measured dipole fields were all slightly higher than the desired 1107 gauss. The maximum value was 1126 and the minimum was 1115 gauss, nn the average, the fields were about I\% high; however, the effective magnetic length was, on the average, about 3.5\% longer than desired. As a consequence, the optimum energy for transmission through the achromat is $46 \mathrm{MeV}$ rather than $45 \mathrm{MeV}$.

The measurements of the quadrupoles showed that the integration of their gradients over their length gives an effective $B^{\prime} \times$ leff from 1.379 to 1.400 Tesla. This compares with a design objective of 1.36? Tesla. Thus, the achieved gradient $x$ length products are about $2 \%$ high on the average. This is consistent with the error in the dipoles. The trim coils will provide about $4.5 \%$ adjustment in effective gradient.

The dipoles and quadrupoles were located within the achromat assembly in such a way to distribute the magnetic errors to minimize their influence. 


\section{DESIGN DRANING}

The drawings for the vernier and achromat are listed in the Appendix. 
Permanent magnet styments were measured for magnetic moment and direction of magnetic axis at LBL. An example of data from these measurements is shown in Table 4. The MAGID nulubers identify the segment and indicate its type and position by the first three digits. For this example, the first w.Iree digits of MAGID indicates that these segments are for the short quadrupoles and for positions 1 and 2. These positions are shown on the design drawings. At these positions, the angles THTAXY should be 180 and 135 respectively. As may be seen from the table, the angles deviate significantly from the desired values. It is aiso clear that there is a significant variation to the magnetic moments $M X Y$ and $M Z$.* Ideally, $M Z$ should be zero. It was anticipated that there would be significant variation inspite of steps taken to minimize the scatter in magnetic properties and orientation of magnetic axes. Because of these expected variations, extra segments were obtained so that, through a selection process, the best arrangement of segments could be madu. Segments with large deviations would be rejected. Values of $M I$ were generally igno ed, even though a large value would indicate some fabrication error.

Using a rather simple algorithm, the segments were assigned to a specific quadrupole or dipole in such a way as to minimize the variation in total magnetic moments for each type of element and to minimize the summation of vertical and horizontal components of the magnetic moments.

\footnotetext{
*The table shows some values from early measurements which should be ignored, The additional digit in front of the MXY value indicates data that has been updaced.
} 


\section{TABLE 4}

DATA FEINTED FREM SCSTES DAFA FILE: AD2. DE1GCD.SRT

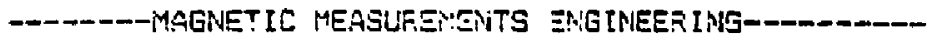

MAGNETIC MOMENT' SORT ING

PAGE 1

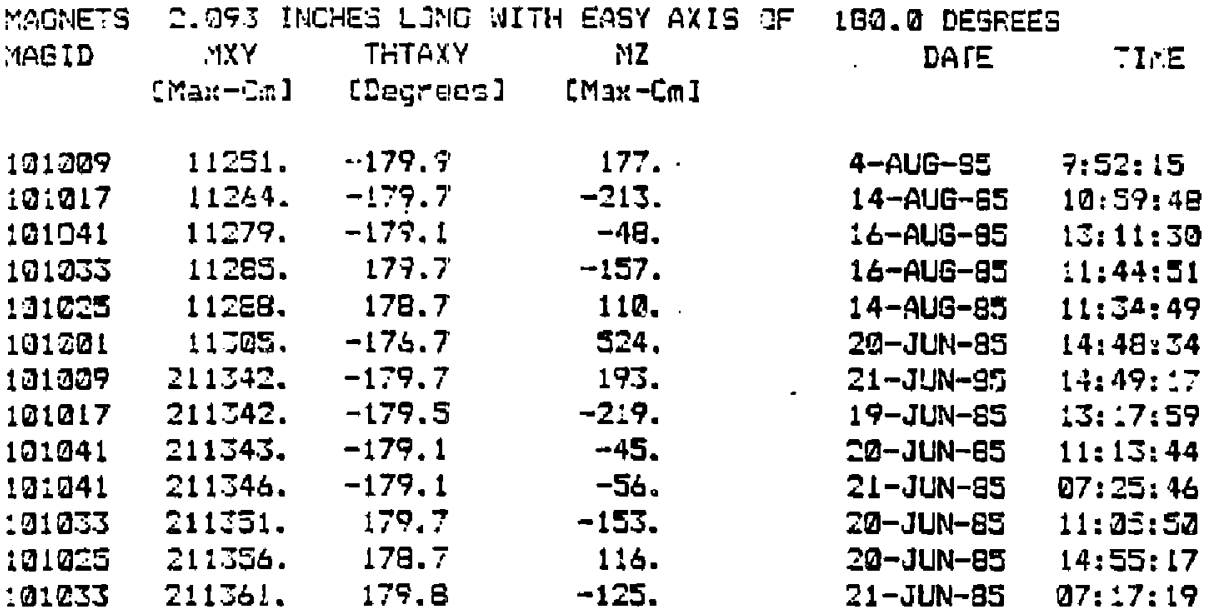

MAGWETS

2. Q9S INEHES LONG WITH SASY AXIS OF

135.0 DEGREES

$$
\text { MYY THTAXY } \$ 2
$$
[Max-Cn] [Degress] [Man-En]

DATE

TINE

\begin{tabular}{|c|c|c|c|c|c|}
\hline 102026 & 11230. & $: 34.5$ & -128. & 14-ALG-95 & $11: 47: 03$ \\
\hline 1,22034 & 11267. & 136.5 & 239. & 16-AUG-85 & $11: 47: 38$ \\
\hline$: 32013$ & $1233 \%$ & $: \div 7 .:$ & -231. & 19-JUN-85 & $15: 26: 27$ \\
\hline 122302 & :1521. & $: \because 3.2$ & -250. & $14-A L G-95$ & 09:23:31 \\
\hline$: 020: 2$ & 11387. & 132.9 & 593. & $=1-J U N-35$ & $D E: 11.54$ \\
\hline$: 220 \div 2$ & 11419. & 132.4 & $-48 i$. & $: 4-406-35$ & $13: 15: 39$ \\
\hline $2=2+2$ & $: 1419$. & 132.6 & -487. & 16-AUG-65 & 1.5: $20: 79$ \\
\hline 30013 & :11372. & .3 .0 & 53. & $2 Q-J U N-35$ & $11: 28: 57$ \\
\hline :322:4 & $21: 513$. & 130.2 & .56. & $1 \div-J U N-85$ & $14: 04: 23$ \\
\hline :2agsi: & $21 ! 331$. & 135.1 & 354. & $20-. J L N-85$ & $68: 4 \geq: 59$ \\
\hline 102026 & 211369. & 1.3 .4 .6 & -123. & 17-JUN-95 & $: 3: 31: 12$ \\
\hline 132202 & 211400. & :35. & $-25 ?$. & 22-JUN-85 & $11: 20: 47$ \\
\hline $10=3.12$ & $211=42$. & 1.32 .7 & $-4 a 2$. & 17-JUN-85 & כצב:14:11 \\
\hline 02042 & $21: 543$. & 135.5 & -407. & 19- JUN-89 & $14: 17: 15$ \\
\hline
\end{tabular}

$-13-$ 
Ideally, the sumnation of horizontal and vertical components should be zero. Because the angular orientations deviate from the ideal values and the mag" stic moments are not unifom, the vector sumations do not yield zero values.

The final assignments for segments for the short quadrupoles is shown in Table 5. The segments for this table were presorted to eliminate those with the greatest deviation in angle or moment. The segments selected have an average magnetic moment, MXY, of 11311.9 with a standard deviation of 52.97. This is less than 0.5\%. Each segment can be used in any of two places and in efther quadrupole ( $S 1$ or $S 2)$. The ideal positions for the segments are those which give the lowest average vertical and horizontal component. The segments are systematically moved from position-to-position to bring these averages to their lowest values.

The assignments of segments for the long quadrupoles are shown in Tables 6 through 9. The method for selection is the same as that for the short quadrupoles explained above. 


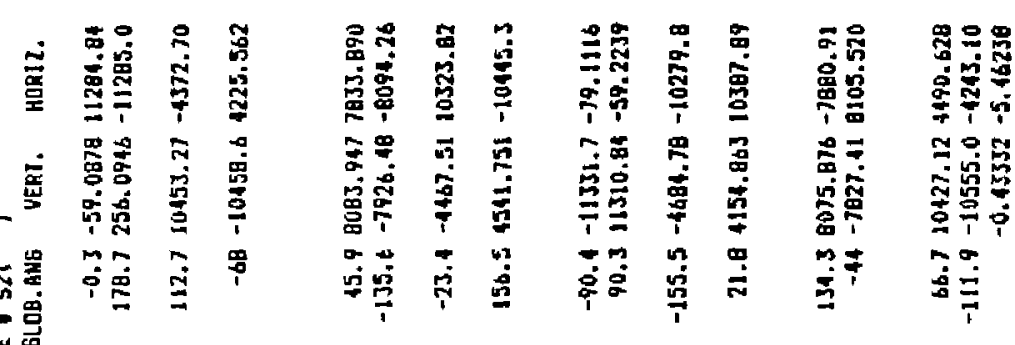

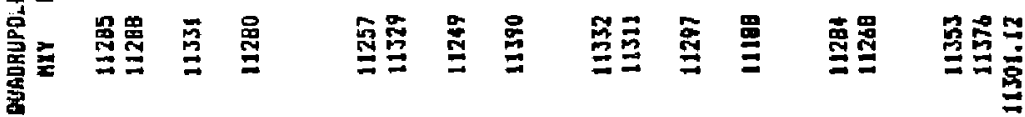

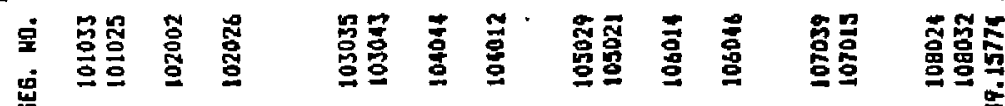

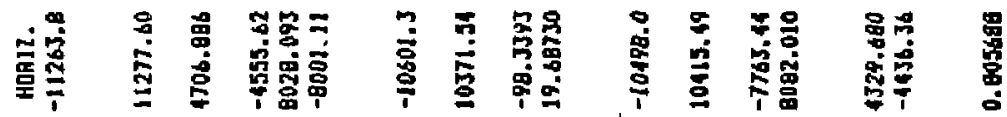

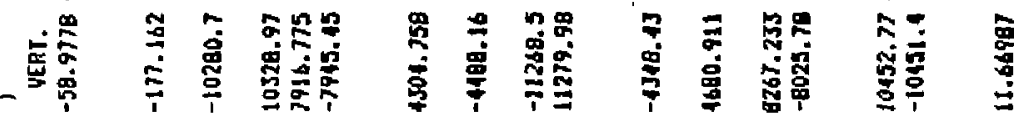

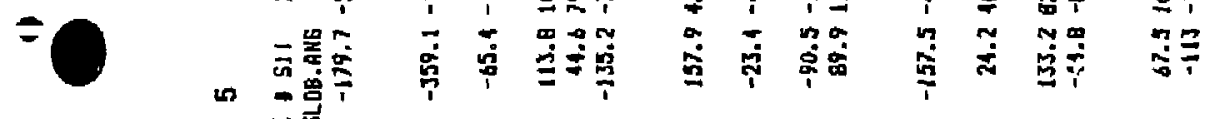

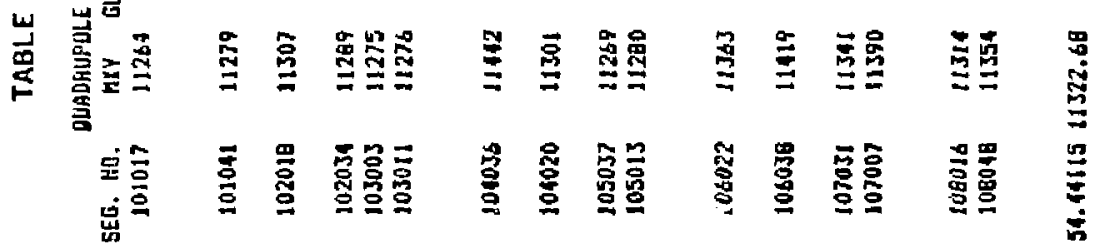

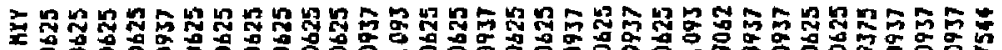

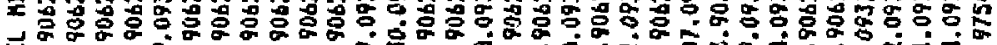

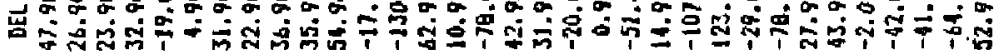

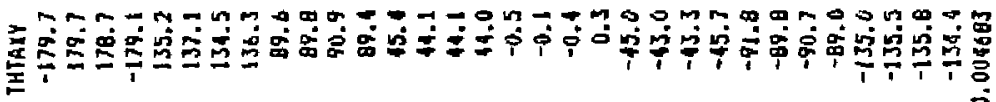

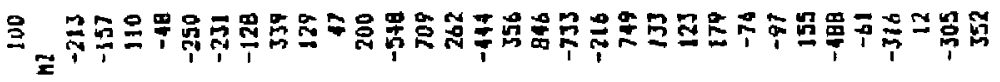

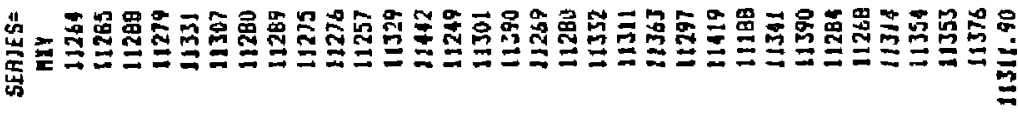

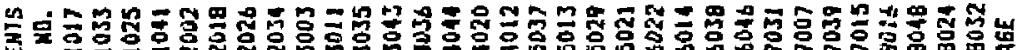

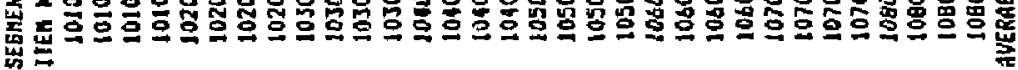




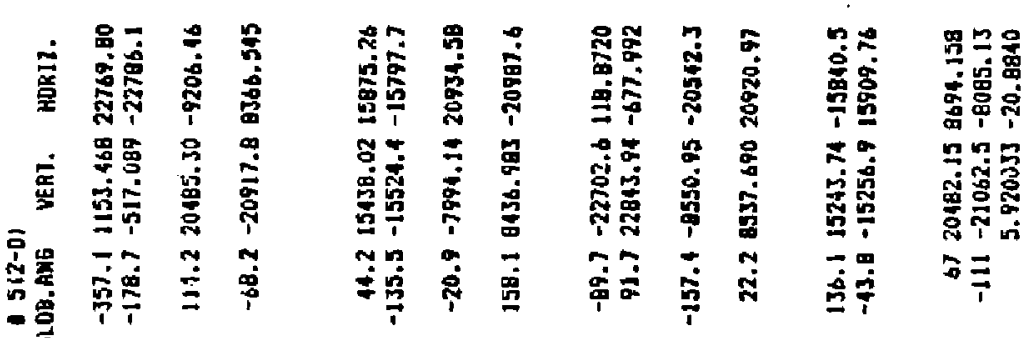

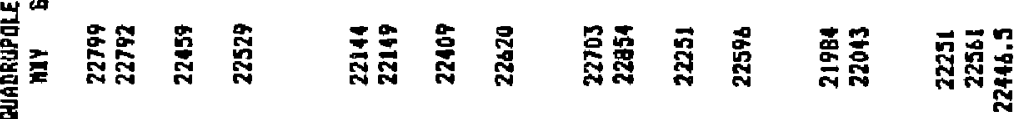

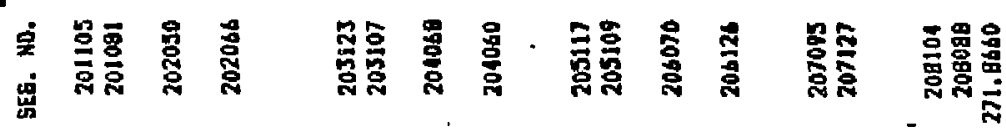

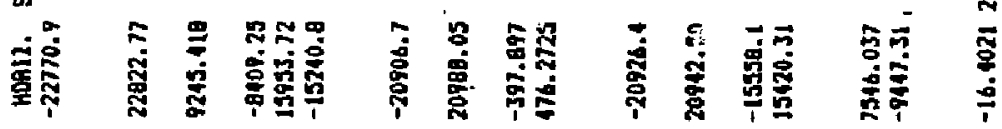

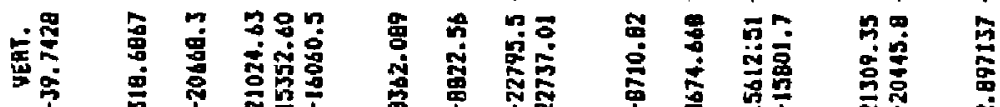

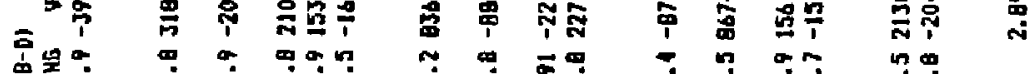

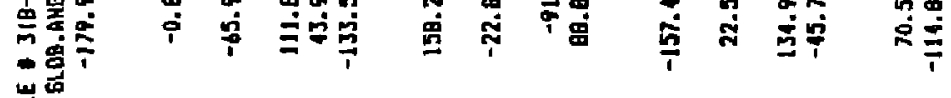

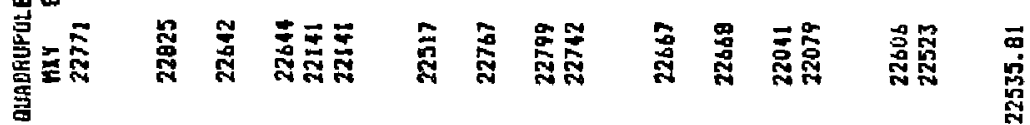

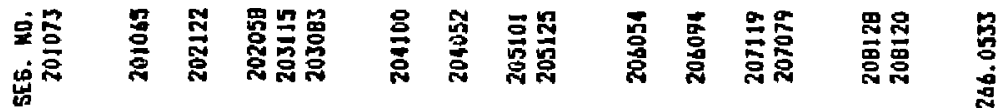

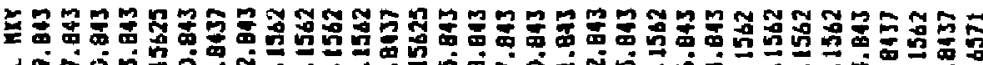

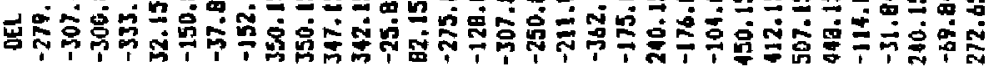

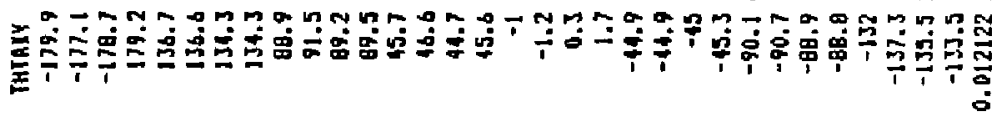

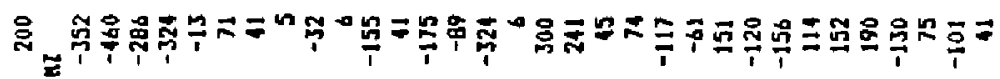

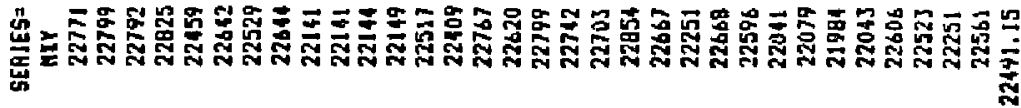

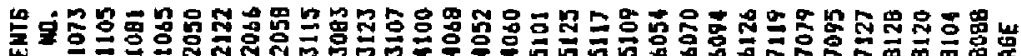
娄 


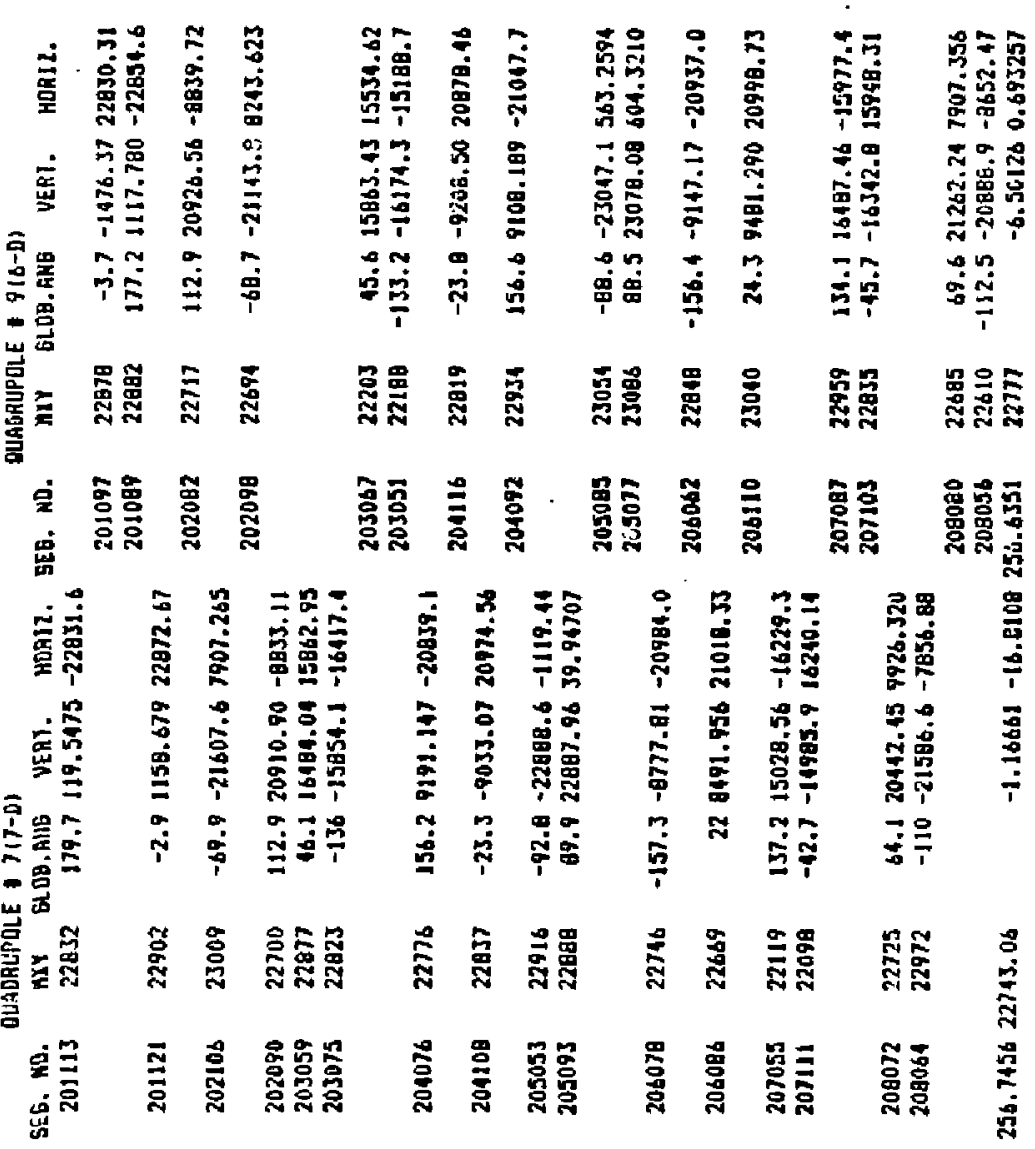

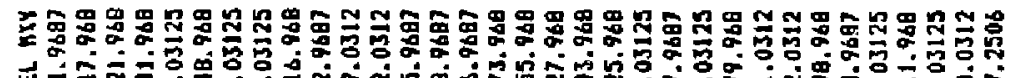

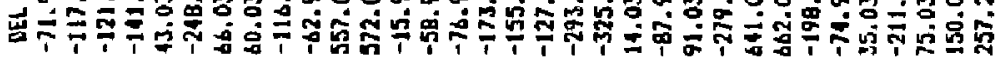

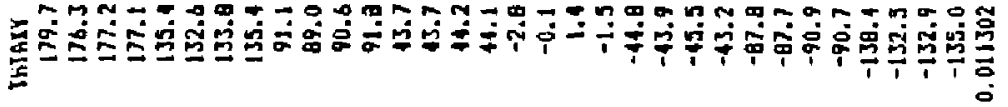

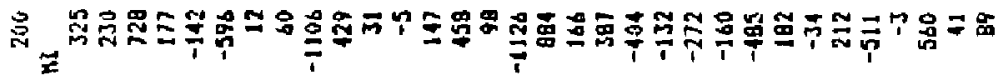

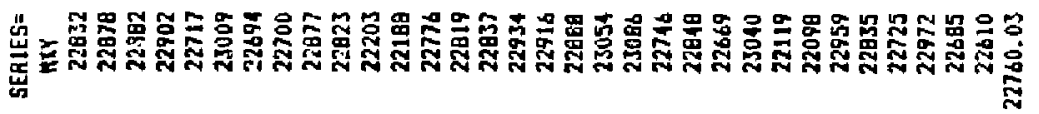

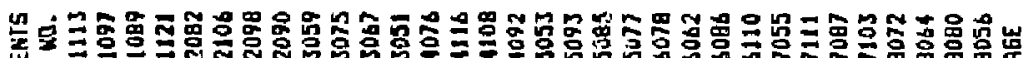

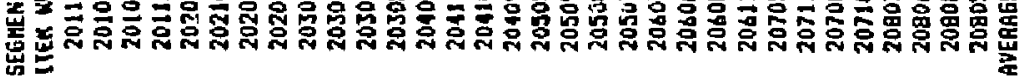




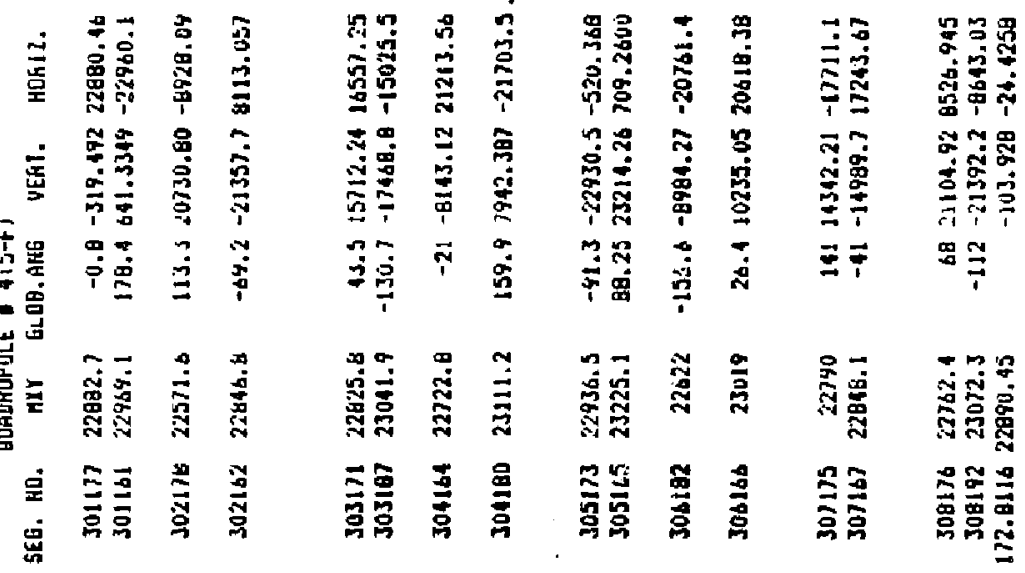

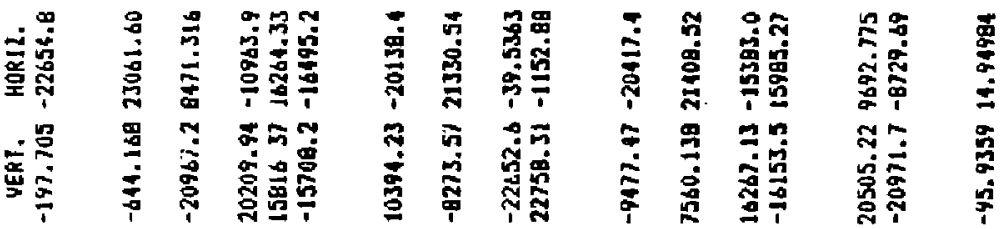

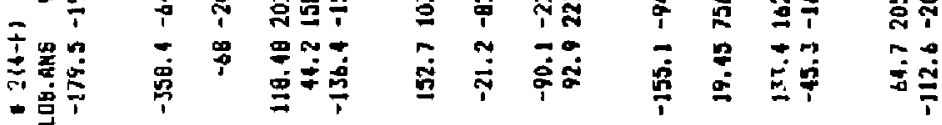

4

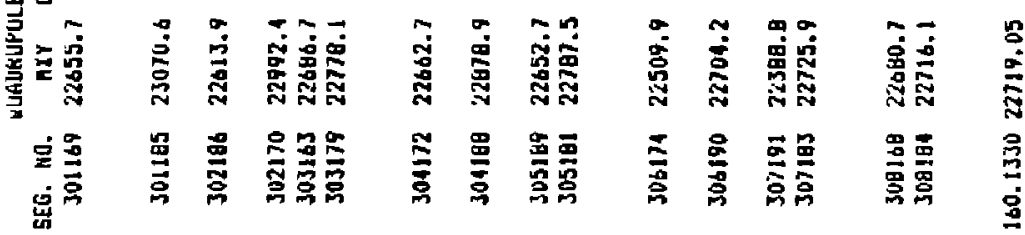

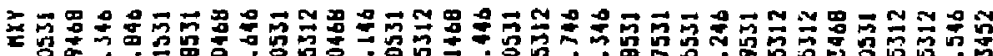

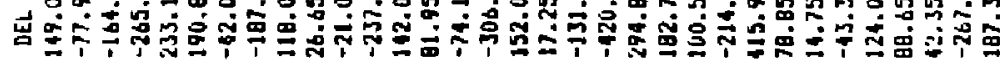

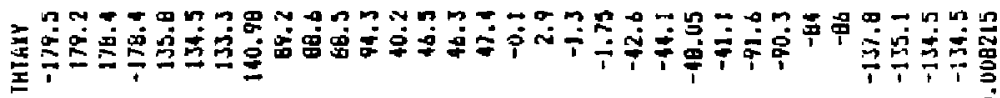

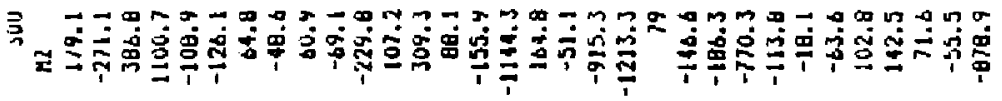

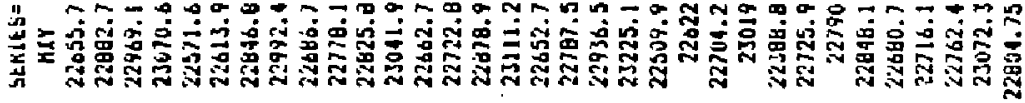

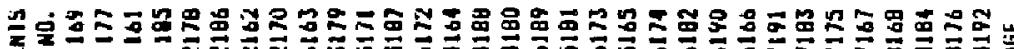

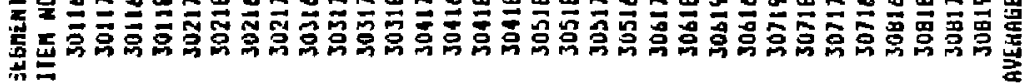


TABLE 9

SEGHENTS SERIES:

IIER KO. MIY

$301209 \quad 22933$

$301193 \quad 22707$

$301217 \quad 22533$

30112922936

$102146 \quad 22656$

$302138 \quad 22600$

$502210 \quad 22793$

$302130 \quad 22951$

$303219 \quad 22692$

$303139 \quad 22469$

$303211 \quad 22722$

J03131 22417

304148 - 22519

$\begin{array}{lll}\dot{0} & 304132 & 22581 \\ & 304140 & 22629\end{array}$

$\begin{array}{ll}304140 & 22629 \\ 304204 & 22955\end{array}$

$30+204 \quad 22955$

J05221 22554

$305197 \quad 22504$

30520522590

$305213 \quad 22669$

$306222 \quad 22597$

30615B 22630

306142 2268?

$306150 \quad 22747$

30721522763

$307151 \quad 22722$

$307223 \quad 22764$

$3071 \div 5 \quad 22853$

$308216 \quad 22545$

$308200 \quad 22535$

$306160 \quad 22577$

308224 22629

AUERAGE 22676.21
MI THJAXY DEL ALY SEG. HO.

$\begin{array}{lll}-160 & -178.9 & -256.781 \quad 301208\end{array}$

$416-178.5-230.781$

$20 \quad-179.6 \quad 113.2187$

$354 \quad-178.8-259.781$

$147 \quad 136.920 .21875$

$-393 \quad 137.776 .21975$

$134.9-116.781$

$135.8-274.781$

$90.7-15.7812$

QB. 2 207.219)

09.6-45.7812

91.3259 .2197

$+4.7157 .2187$

45.595 .21875

$14.6 \quad 17.21675$

14.6 -278.781

$-0.5122 .2187$

$-0.7172 .2107$

-0.2 6b. 21875

$-0.3 \quad 7.21875$

$-45.179 .21875$

$-43.146 .21875$

$-44.2-10.7812$

$-44.1-70.7812$

$-6 \mathrm{~d} .4-66.7862$

$-94.7-45.7912$

$-91.1-67.7912$

$-91.4-156.791$

$-135.4(31.2197$

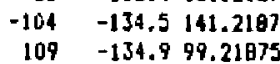

$-135.747 .21875$

0.006454146 .3659154 .220822664 .75

302138

22600

$304140 \quad 22629$

$308200 \quad 22535$
GUADRUPOLE $B(3-F)$

$22933-.78 .9-440.254-22928.7$

$301: 29 \quad 22936-358.8-490.33522930 .96$

$-64.8-20449.09622 .611$

$302130 \quad 22951 \quad 113.321079 .26-9078.16$

$303219 \quad 22692 \quad 45.716240 .4915849 .43$

$30315922469-136.3-15381.0-16379.1$

$304140 \quad 22519 \quad 157.28726 .465-2.759 .4$

$-22.9-8805.4020845 .50$

$505221 \quad 22554 \quad-90.5-2255 \$ .1-196.818$

$305197 \quad 22504 \quad 89.322502 .32274 .9313$

$306222 \quad 22597 \quad-157.6-8611.04-20991.9$

$306142 \quad 22487 \quad 23.38973 .74020836 .79$

$367215 \quad 22763 \quad 136.6 \quad 15640.17-16539.0$

s07ist $22722 \quad-45.7-16261.915869 .39$

$308216 \quad 22545 \quad 67.120768 .128772 .799$

67.120768 .128772 .799
$-112-20894.0-8411.75$

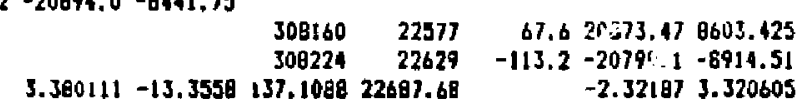

OUAORUFOLE I B BI-FI

$22907-358.5599 .635322699 .15$

$301217 \quad 22533 \quad-179.6-157.309-22532.7$

$302146 \quad 22656 \quad 114.420632 .44-9359.29$

'J02210 $22793-67.6-21073.1$ 8685.737

$303211 \quad 22722 \quad 44.6 \quad 15954.3216176 .65$

$303131 \quad 22417-133.7-16206.7-15191.5$

$304132 \quad 22581 \quad-22-8458.9920936 .73$

$30420422955 \quad 157.36958 .473-21176.8$

$305205 \quad 22590 \quad-90.2-22589.8-76.8538$

$30521322669 \quad 99.722668 .68118 .6940$

$306158 \quad 22630 \quad-155.9-9240.51-20657.4$

$306150 \quad 22747 \quad 23.49033 .92326976 .16$

$307223 \quad 22764 \quad 133.916402 .62-15784.5$

$307135 \quad 22833 \quad-\$ 6.4-18535.0 \quad 15746.00$

$308160 \quad 22577 \quad 67.625 .53 .470603 .425$

$-2.321873 .320605$ 
The assignment of segments for the dipoles are shown in Tables in through 14. The process for selection is similar to that explained for quadrupoles except that any segment can be used in only one position, but in any dipole. Thus, the allowable trade-offs are fron dipole-to-dipole. Because the segments were received in batches sufficient for assembly of only a few dipoles, the extent of the trade-offs was limited. As was the case with the quadrupoles, segments with large deviation were eliminated early on and a batch of 32 segments were sorted into two dipoles each with about the same total magnetic moment. 


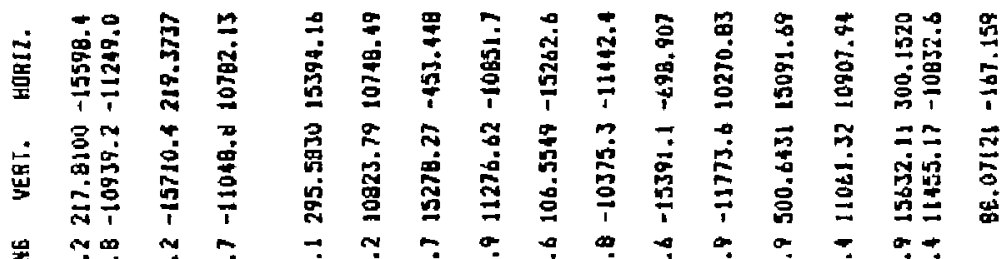

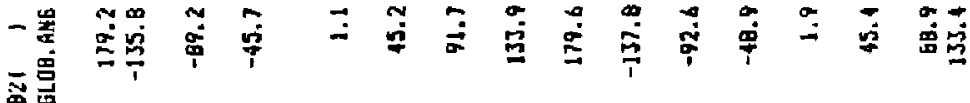

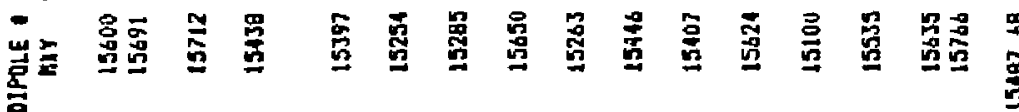

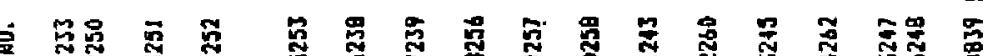

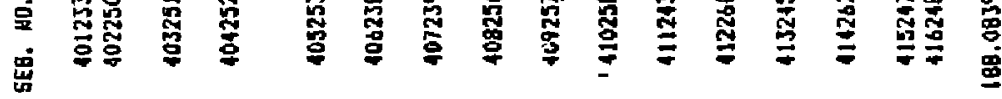

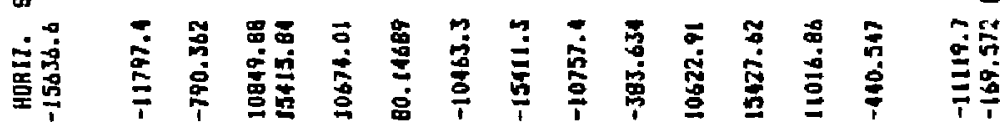

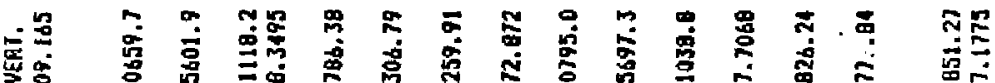

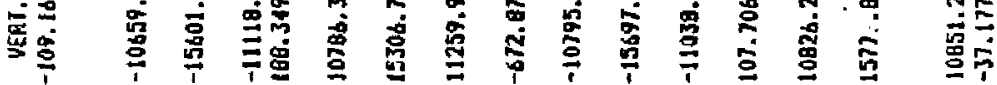

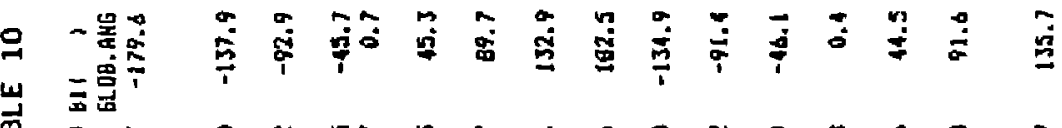

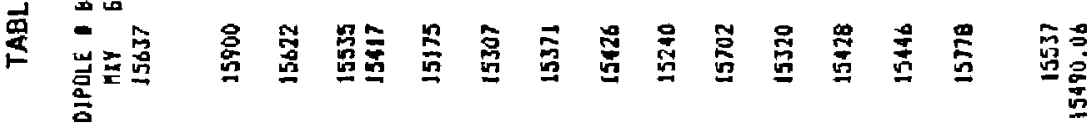

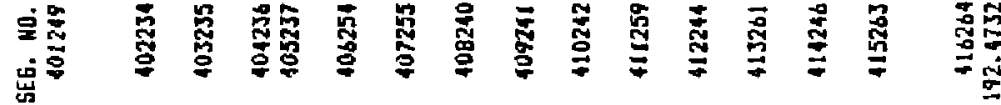

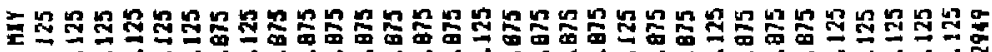

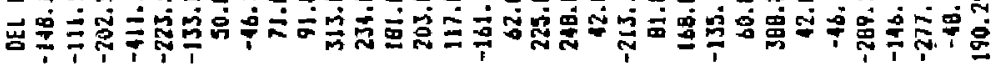

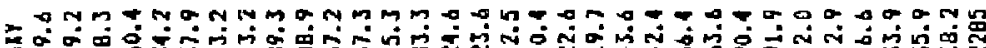

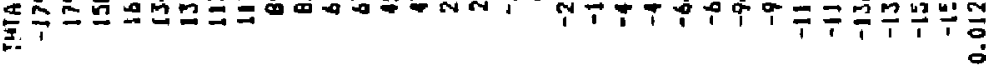

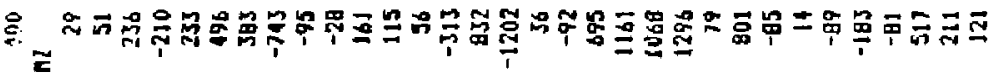

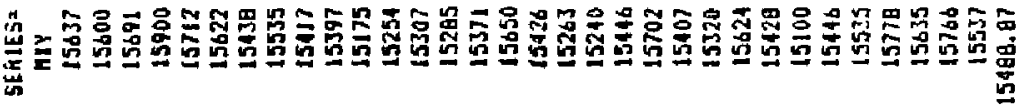

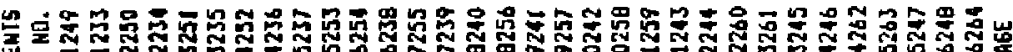
แู่ 


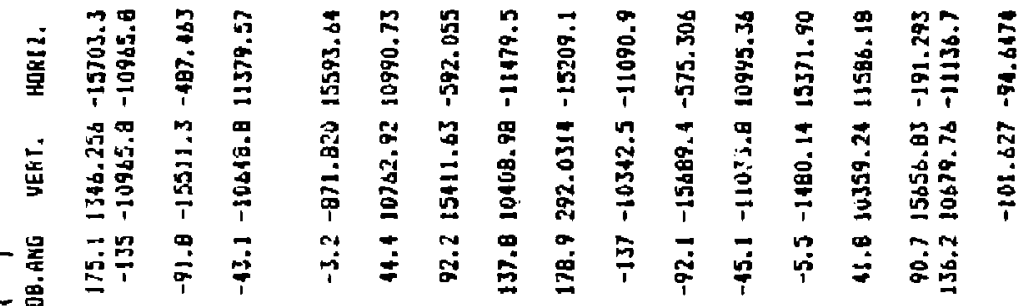

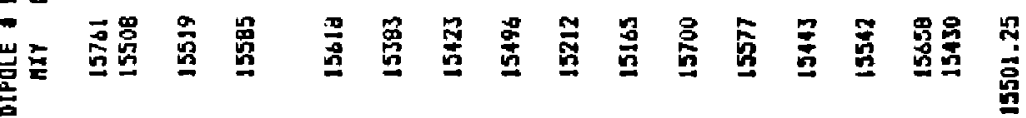

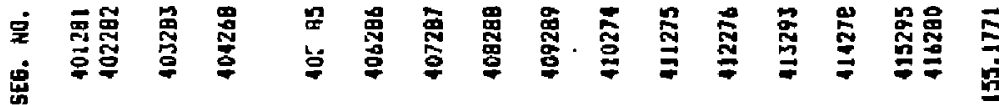

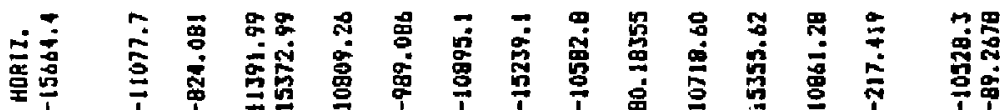

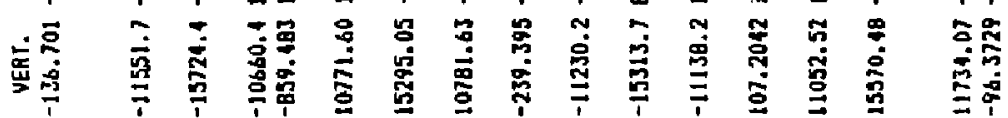
置

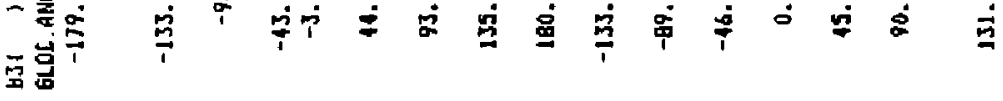

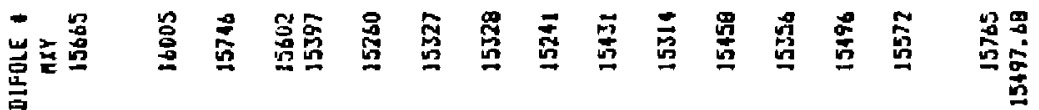
웅 总

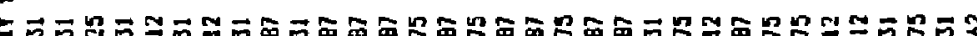

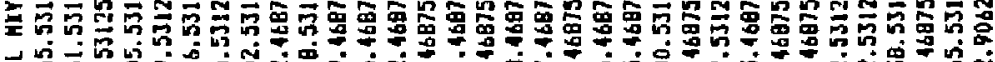

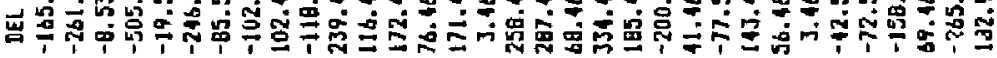

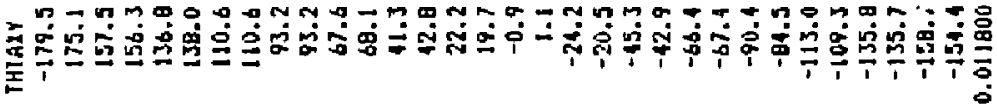

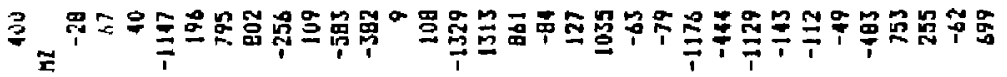

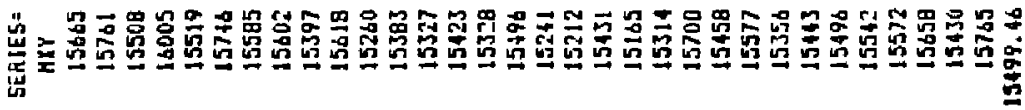

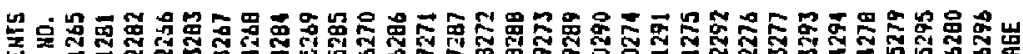

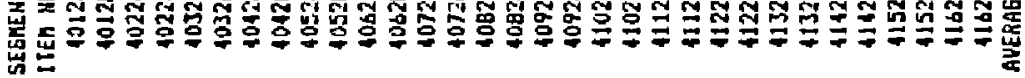




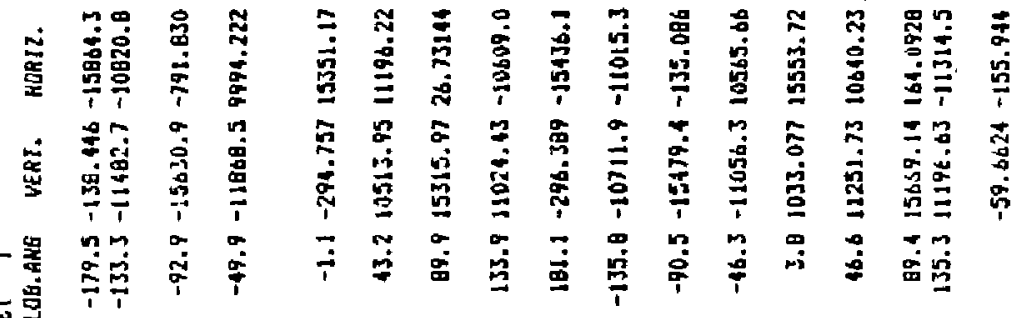

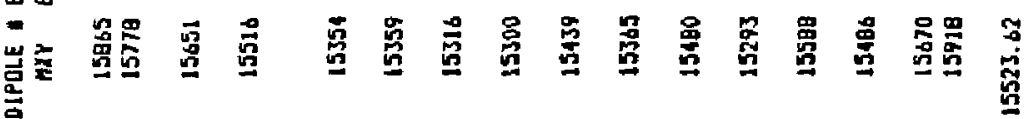

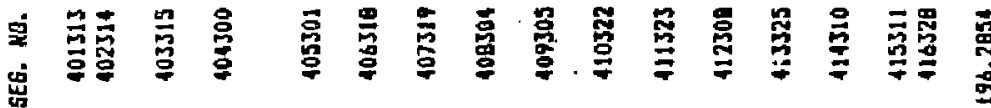

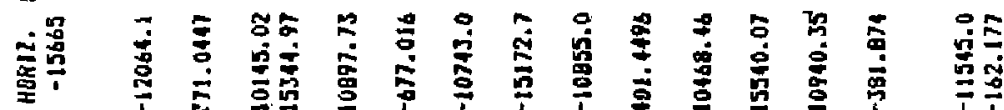

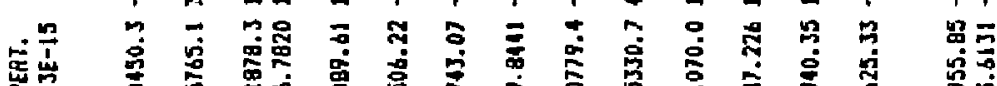

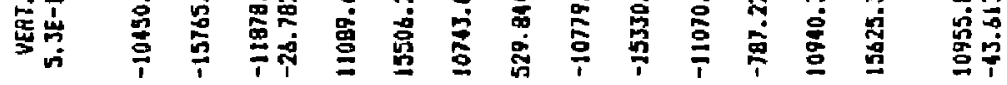

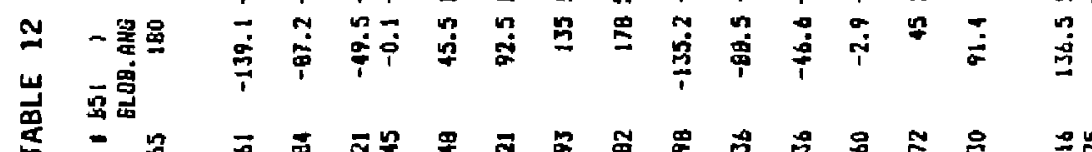

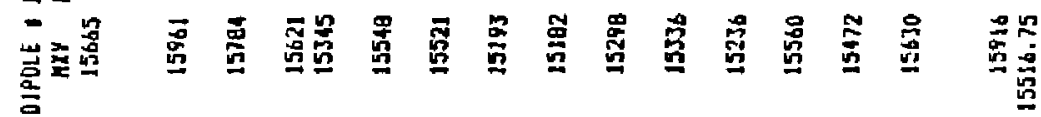

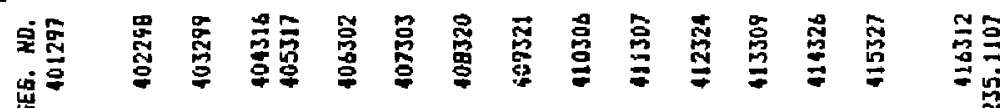
फ

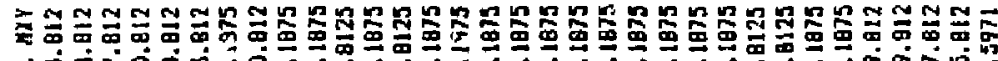

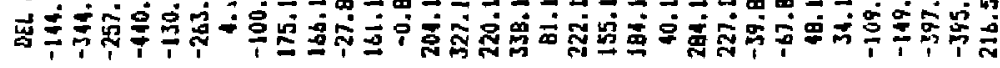

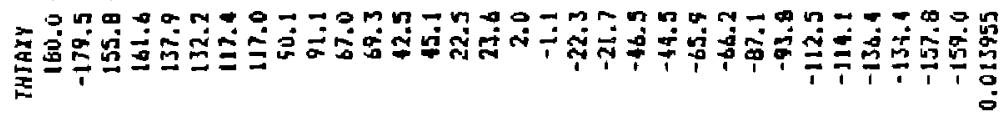

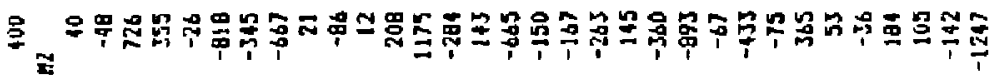

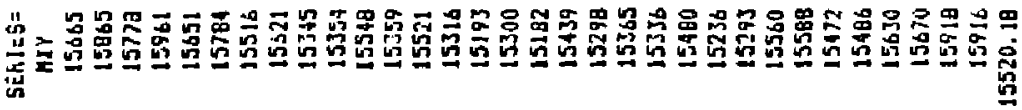

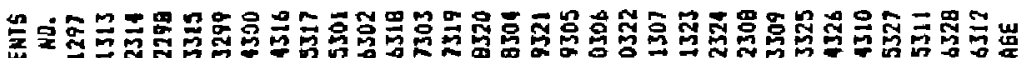

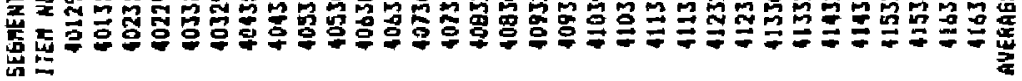




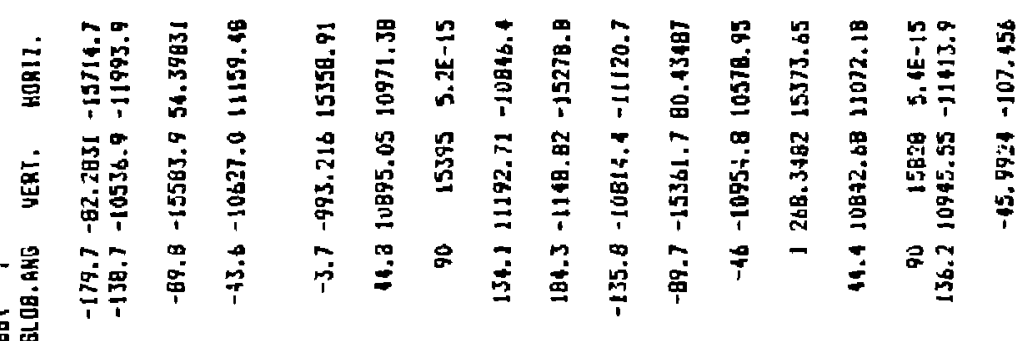

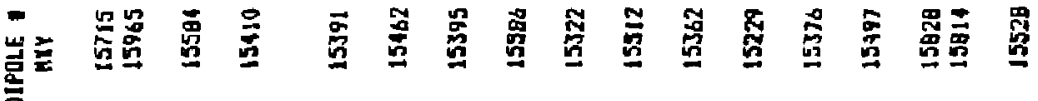

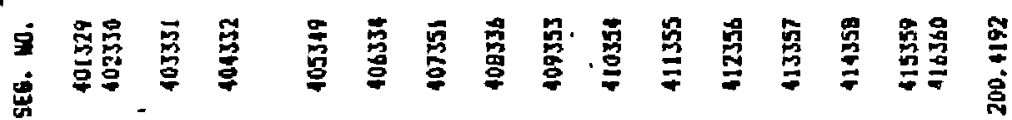

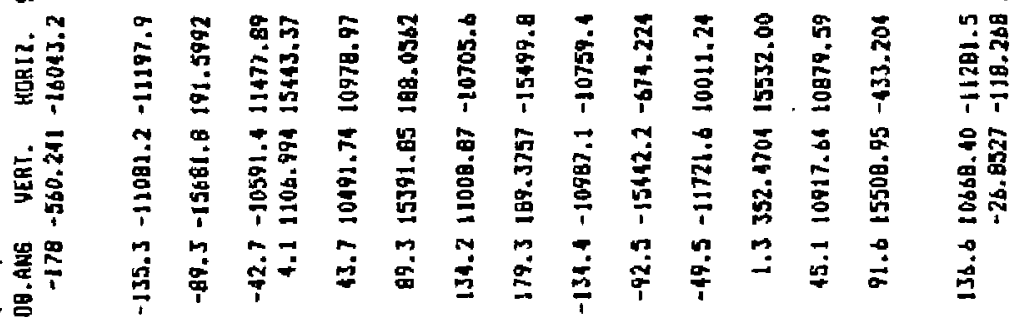
山 $\stackrel{\vec{m}}{\underline{c}}$

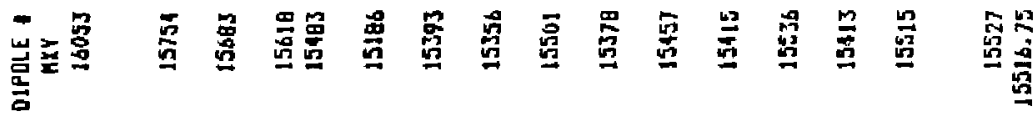

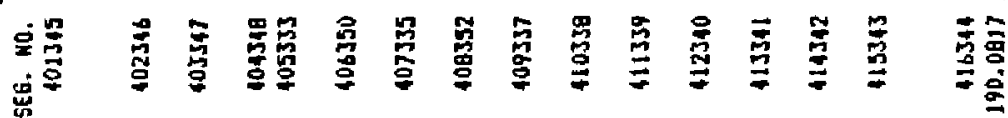

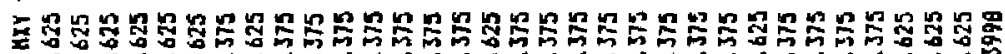

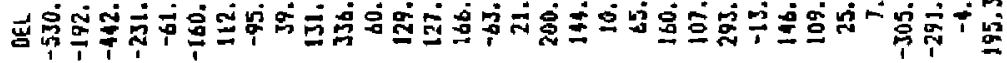

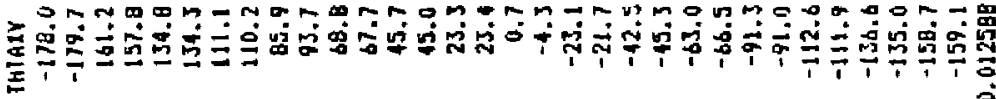

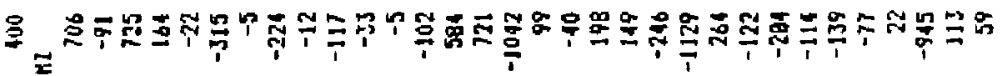

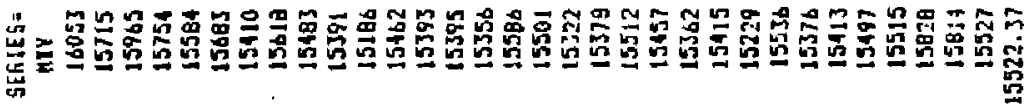

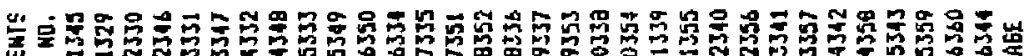

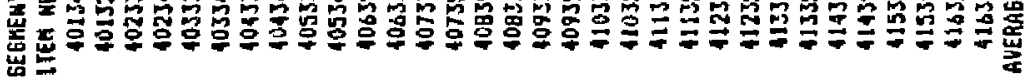




\section{TABLE 14}

\begin{tabular}{|c|c|c|c|c|c|c|c|c|}
\hline SWETS & & 40 & & & & JIERLE i & $\$$ & \\
\hline En yn & $I I Y$ & R? & TAXY & JEE WIY & AES. WA. & nIY & 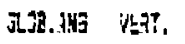 & 4tinl. \\
\hline 20!36! & 15855 & $-2 \pi 6$ & 179.7 & $-545,875$ & $40: 36 \mathrm{i}$ & 45875 & :79.7 85.1118.5 & $-i 5979.7$ \\
\hline $4913<5$. & 6653 & 706 & -178.0 & -325.275 & & & & \\
\hline $40 \div 3000$ & 15965 & 725 & 81.2 & -137.875 & & & & \\
\hline $40 * 662$ & $1579 \pi$ & -157 & 157,0 & -259.879 & 402362 & 15787 & $-134.5-11260.0$ & -11065.2 \\
\hline 6053510 & I5580 & -22 & 134.8 & -56.879 & & & & \\
\hline 403563 & 15713 & 237 & 137.0 & -185.875 & 103353 & 15713 & $-92-15703.4$ & $-4+8.375$ \\
\hline CQ13520 & 15410 & -5 & 111.1 & 117.125 & & & & \\
\hline 404564 & 15478 & $\Pi 7$ & 110.2 & 29.125 & 104364 & 15198 & $-42.7-10510.1$ & 11589.70 \\
\hline 105365 & 15376 & 78 & 97.6 & 191.125 & 605365 & 15776 & $-7.6-5033.57$ & {$[5260.9]$} \\
\hline 2053490 & 15391 & $-\{117$ & 73.7 & 136.125 & & & & \\
\hline 906336 & $\$ \$ 47$ & 327 & 69.6 & 50.125 & 106386 & 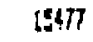 & 12.9 !6535.5! & $1: 3 \% 7.56$ \\
\hline 4063500 & 15196 & -33 & 58.8 & 341.125 & & & & \\
\hline 407767 & isfig & 870 & 13.8 & 9.125 & $40756 ?$ & i5518 & 91.215514 .59 & $-\$ 24.964$ \\
\hline 1073510 & 15395 & 304 & 15.0 & 152.125 & & & & \\
\hline 409368 & 1.5893 & -15 & 21.4 & 244.125 & 4087398 & 15243 & $136 .: 10597.26$ & $-[: 912,1$ \\
\hline+093520 & 15356 & $72 t$ & 33.3 & 171.125 & & & & \\
\hline 409769 & i:4489 & -315 & 3.0 & 69.25 & 409359 & 15458 & 177309.3692 & $-154: 6.8$ \\
\hline 4973550 & 1573 & -40 & -4.3 & 205.125 & & & & \\
\hline 410570 & $: 5513$ & 25 & -21.7 & 14.125 & $4 \log 70$ & $155: 3$ & $-135.8-108: 5.1$ & $-! ! 121.4$ \\
\hline 4105540 & 15512 & $: 49$ & $-2 ! .7$ & 19.175 & & & & \\
\hline$\$ 11371$ & $15: 73$ & 187 & -43.5 & 254.125 & $41157 !$ & :527J & $-41.5-15267.7$ & -399.300 \\
\hline 4113550 & 15362 & -1129 & -45.3 & 165.25 & & & & \\
\hline 112372 & $: 5458$ & -359 & -71.4 & 69.125 & $4: 232$ & 15458 & $-41.6-1016: .7$ & 11649.58 \\
\hline $1123: 60$ & 15259 & -122 & -56.5 & 298.125 & & & & \\
\hline 115073 & 1549 & 167 & $-94,0$ & 70.125 & $4 ! 3373$ & {$[5457$} & +1078.225 & 15469.5 \\
\hline 1135570 & $15: 76$ & -114 & -71.0 & $\$ 91.125$ & & & & \\
\hline 261374 & 1849 & -115 & $-1: 0.7$ & 29.195 & 4145.4 & 15196 & $43.21 .699 .1 \%$ & 11.99 .55 \\
\hline 414380 & $1: 997$ & -77 & $-11: .9$ & 71.155 & & & & \\
\hline 41575 & $: 979$ & $-!: 23$ & $-132,0$ & $-35 \div .875$ & 415374 & :5779 & $87: 5757.37$ & 3AE. $309 j$ \\
\hline+153590 & & -795 & -135.0 & -500. & & & & \\
\hline 46354 & & 59 & -159.1 & $0.12 \mathrm{E}$ & & & & \\
\hline & 15914 & - jut & -158.1 & -396.075 & 416,376 & 15914 & los.d :atist.44 & $-! i=70.1$ \\
\hline WERAGE & 5527.12 & & $0.014: 30$ & 217,4325 & 91.3693 & $: 5354.68$ & 25,92794 & 6.484777 \\
\hline
\end{tabular}




\section{MAGNETIC MEASUREMENT ON ASSEMBLIES}

The dipole and quadrupole assemblies were measured at LBL. The data on the quadrupoles is summarized in Table 15. The values of $R$ leff shown in the table should be compared to the design objective of 1.362 for the long quadrupoles and 0.681 for the short quadrupoles. All of the units show a slight offset of the SC axis. This offset translates to a superimposed dipole. Taking into account the direction and agnitude of this offset for each quadrupole, it was possible to arrange the distribution of quadrupoles to minimize the beam deflection due to this very small of fset.

The data for the dipales is summarized in Table 16 . The values 8 leff should be compared with the design objective of $100996(.1107 \times .09)$. The maximum angular error is about 1 degree. The quadrupole content is small but the sextupole content is perhaps higher than desired. To minimize the influence of the sextupcie content, the dipoles were arranged to distribute this component along the beam axis. 
TABLE 15. QUADHUPOLE ASSEMBLIES MEASUREMENTS SUMUARIZATION

MATASET

QUAD ORIENT

ID

$1106 \mathrm{CO}$
$1106 \mathrm{Cl}$
$1106 \mathrm{C2}$
$1106 \mathrm{C3}$
$1125 \mathrm{AO}$
$1125 \mathrm{~A} 1$
$1125 \mathrm{~A} 2$
$1125 \mathrm{A3}$
$1106 \mathrm{CB}$
$1106 \mathrm{C} 9$
$1106 \mathrm{DO}$
$1106 \mathrm{B1}$
$1106 \mathrm{B2}$
$1106 \mathrm{HI}$
$1108 \mathrm{BO}$
$1108 \mathrm{~B} 1$
$1108 \mathrm{~B} 2$
$1108 \mathrm{B3}$

110800

1108 II

110802

110813

$1125 \mathrm{CO}$

$1125 \mathrm{C} 1$

$1125 \mathrm{C} 2$

$1125 \mathrm{C} 3$

$1125 \mathrm{k0}$

$1125 \mathrm{~kg}$

$1125 \mathrm{k2}$

$1125 \% 3$

112500

112501

112502

1125113

$\begin{array}{ll}\text { 8D } & \text { Hork } \\ \text { 8D } & \text { Horn } \\ \text { 8n } & \text { Rev } \\ \text { 8I } & \text { Rev }\end{array}$

$1 F$

IF

$1 F$

IF

20

20

20

2II

3F

3F

$4 F$

$4 F$

$4 F$

$4 F$

SF

JF

SF

$5 \mathrm{~F}$

60

6 II

$\delta$ II

6ป

70

71

70

70

85

BI

8D

EN
Norn

Norn.

Rev

kiev

Fev

Rev

Nora

Norn

Nora

nev

Mor"

Nora

Rey

hev

More

Norm

Rey

Rey

Norm

Norfi

Rev

Rey

irora

Hora

Rev

Rev

Hlornt

Norn

Rev

Rev
SC AXIS

OFFSET

(on)

0.16

0.16

0.17

0.17

0.06

0.06

0.04

0.04

0.09

0.09

0.10

0.10

0.05

0.06

0.20

0.19

0.18

0.18

0.01

0.01

0.04

0.03

0.07

0.07

0.04

0.04

0.11

0.10

0.13

0.13

0.15

0.15

0.16

0.16
SC. Axis ANGLE

(de's)

$98.3 \quad 1.379$

$97.9 \quad 1.379$

$-111.9$

$-111.9$

27.2

28.0

$-148.0$

$-106.5$

$-107.0$

67.7

66.1

70.2

$-174$.

43.2

42,8

$-.35 .1$

$-35.0$

153.8

168.0

$-178.8$

174.8

$$
21.9
$$

22.0

$-111.3$

$-111.4$

ก5. 5

85.8

$-123.0$

$-123.8$

$$
\text { E0.0 }
$$

79.7

$-115.6$

$-195.2$

1.379

1.380

1.387

1.386

1.398

1.388

$$
1.374
$$

1.375

1.376

1.377

1. 381

1.386

1.388

1.388

1.389

1.390

1.398

1.397

1.399

1.398

1.400

1.400

$\therefore .402$

1.402

1.100

1.100

1.400

1.403

1.383

1.383

1.384

1.363
E'Lefr

angle

(dies) (óps) (tT-M)

$$
\begin{aligned}
& 4.4 \\
& 4.4
\end{aligned}
$$

$-175.1$

$-175.1$

$\begin{array}{rll}5.5 & 0.100 & 0.083 \\ 5.5 & & 0.083 \\ -174.9 & & 0.056 \\ -175.0 & & 0.056\end{array}$

$-176,0$

$-0.375$

0.124

$-176.0$

5.5

5.4

5.3

$-175.7$

$-0.250$

0.069

$$
\begin{array}{rrr}
5.7 & -0.150 & 0.278 \\
5.6 & & 0.264 \\
-174.9 & & 0.250 \\
-174.9 & & 0.250 \\
& & \\
5.7 & -0.525 & 0.014 \\
5.7 & & 0.014 \\
-176.4 & & 0.056 \\
-176.4 & & 0.012
\end{array}
$$

0.098

$0.09 \mathrm{~B}$

0.056

0.056

$-174.2$

$-174.2$

0.413

0.154

0.140

0.182

0.182

$-173.0$

$-17 \% .4$

0.207

0.207

0.221

0.221

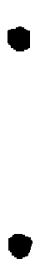

\section{S1 Nora}

S1 Fiev

S2

52
Norm

kiev

$\begin{array}{rr}0.16 & -64.4 \\ 0.10 & 98.1\end{array}$

$0.09 \quad 164.9$

$0.14-173.9$

$-27$.

$$
\begin{array}{rrrr}
0.696 & 4.6 & 0.300 & 0.111 \\
0.696 & -174.2 & & \\
& & & \\
0.594 & 5.7 & -0.025 & 0.082 \\
0.694 & -174.4 & &
\end{array}
$$


TABLE 16. BROBECK PERMANENT MAGNET DIPOLES 22 November 1985

\begin{tabular}{|c|c|c|c|c|c|}
\hline$S / N$ & $\begin{array}{l}B \quad \text { leff } \\
(T-m) \\
\end{array}$ & $\begin{array}{c}B \\
(T) \\
\end{array}$ & $\begin{array}{l}\text { Dipole Dev. } \\
\text { (degree) } \\
\end{array}$ & $\begin{array}{l}\text { Quad. } \\
\text { Relative } \\
\square\end{array}$ & $\begin{array}{c}\text { Sextupole } \\
\text { Relative } \\
\end{array}$ \\
\hline 81 & 0.01030 & 0.1120 & -0.43 & 0.5 & 4.8 \\
\hline 82 & 0.01027 & 0.1117 & -1.14 & 2.1 & 4.5 \\
\hline B3 & 0.01028 & 0.1117 & +1.00 & 2.7 & 4.0 \\
\hline B4 & 0.01028 & 0.1115 & +0.01 & 2.2 & 6.0 \\
\hline B5 & 0.01032 & 0.1119 & -0.74 & 1.2 & 5.0 \\
\hline 86 & 0.01033 & 0.1124 & -0.93 & 1.0 & 4.1 \\
\hline B7 & 0.01034 & 0.1123 & -0.32 & 2.4 & 4.5 \\
\hline 88 & 0.01030 & 0.1117 & -0.46 & 0.6 & 3.4 \\
\hline B9 & 0.01035 & 0.1126 & -1.01 & 1.0 & 2.7 \\
\hline$B 1^{*}$ & 0.01029 & 0.1119 & -0.96 & 0.5 & 4.8 \\
\hline
\end{tabular}




\section{ARRANGEMENT OF ACHROMAT ELEMENTS}

The dipoles and quadrupoles were arranged alang the beam axis in such a way as to minimize the effects of magnetic errors.

The quadrupoles were arranged in the following order along the beam path:

$$
S 2,1 F, 20,3 F, 70,5 F, 60,80, \$ 1
$$

The 52 and 51 quadrupoles were installed in the defocusing positions and the 80 was rotated 90 degrees in order to use it as a focusing unit. The 70 quadrupole was rotated 180 degrees to balance out ts dipole content. Because the 4f quadrupole had the largest offset error, it was set aside as a spare,

The dipoles were arranged along the beam axis in the following order:

$$
B 1, B 4, B 8, B 3, B 9, B 2, B 6, B 5
$$

Because $B 7$ had the highest combination of quadrupole and sextupole components, it was set aside as a spare.

The arrangement of the dipoles and quadrupoles to minimize the effects of errors in dipole, quadrupole, and sextupole content was based on a simplistic attempt to balance the dipole and quadrupole vectors. It was not rigorous, but the arrangement, in any case, needs to be documented and is, therefore, shown here. 


\section{TESTS AT BROBECK CORPORATION}

Prior to delivering the vernier and achromat to Lawrence Livermore National Laboratory (LLNL), the units were tested for vacuum leaks. In addition to leak testing, the vernier coils were operated at design current in vacuun for a short period of time. Calculations show that full current can be sustained for up to two minutes without cooling. Since heat transfer inside the vacuum chamber is difficult, operation times longer than two minutes at full current, even with the cooling water on, is not advised. At half current, operation should be limited to eight minutes.

Test on the achromat other than leak testing consisted of operating the trim coils at part load. No adverse heating occurred.

Since the polarity of pemanent magnet devices is fixed and cannot be reversed by simply switching the coil leads, a polarity check using a compass and a current-carrying tensioned wire was used at the time of assembly to verify proper polarity for steering an electron beam. 


\section{RECOMMENDATIONS FOR POWER SUPPLIES}

The power supplies and expected field for the vernier coils and achromat trim coils are shown in Table 17. The trim coil current of 4.35 amp to produce a gradient of $64.3 \mathrm{G} / \mathrm{cm}$ corresponds to a $4.6 \%$ adjustment relative to the design point gradient. 


\section{TABLE 17}

FOLER SLFFLLES AUUE EXFELTUD FIELDS FOR EEAM DOEETOF COMFOMENTS

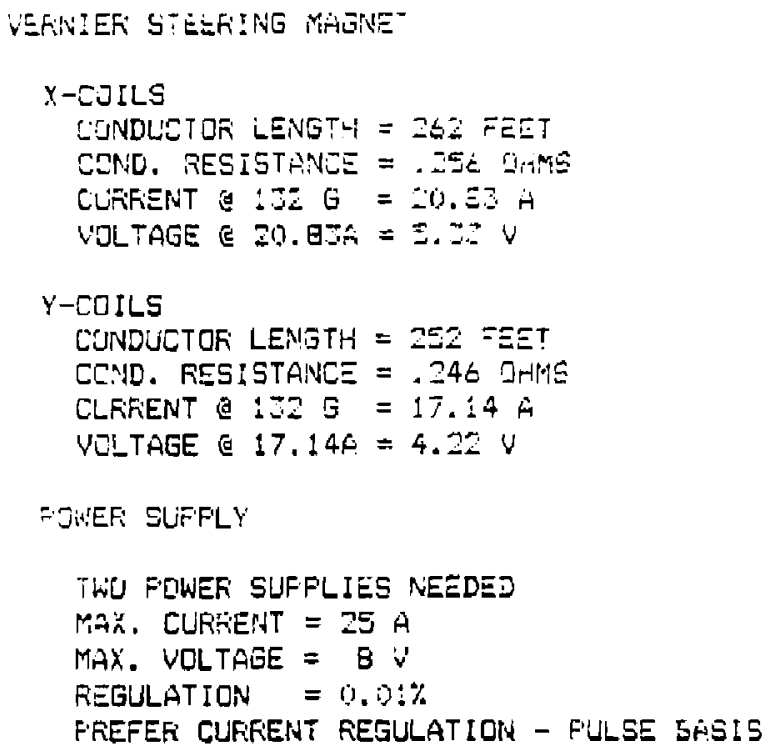

\section{ACHFIOMAT TRLM COILS}

CONDUCTDFS 18 GAGE. SG TUFINS/SLQT

CONCUCTOF LENETH $=24 \%$ FEET

COND. FESISTANCE $=15.0$ OHHS TOTAL

CONNECTIONS ALL COILS IN SEFIES

CURFEENT $\cong$ a $4.3 \mathrm{G}, \mathrm{Cm}=4.35$ AMFE

MAX. CURRENT TOTAL $=4.5$ AMF'S

MAX. DC VOLTAGE $=67.5 \mathrm{~V}$

INDUCTANCE $\quad=6.0 \mathrm{~m}: \mathrm{ri}$

TIME CENSTANT $\quad=0.40 \mathrm{~m} \mathrm{~s}$

FOWER SUFFLY

MAX. CUFTEENT = O HFIFS

MAX. VOLTAGE $=1.30 \mathrm{~V}$

FIEGULATION $=0.1 \%$

PREFER CUFFENT REGILATION - FLLLSE GAÖIS 


\section{RECOMMENDED TEST PROGRAM}

The beam director components require verification of their operating characteristics at high current density where space charge and ionization of residual gas may have significant influence on performance. The electron beam at ATA can provide sufficient current to permit meaningful tests to be performed. It is desired that the beam transport characteristics of the achromat and vernier steering magnet be measured over a range of beam current densities and residual gas densities.

Because a tightly focused beam at high current can produce a current density sufficiently great to cause physical damage to the ciroonents if the beam strikes the wall, it is advisable to make preliminary measurements at low current and small spot sizes. A collimator and beam scraper upstream of the components will be required to limit the beam diameter. It is also proposed that prior to testing on ATA, tests using a low current beam from the RF linac be perfomed to characterize the achromat and vernier steering magnet. Measured characterics for the low current tests should be campared with calculated characteristics based on the magnetic measurements and physical arrangement.

For all úf these tests, it will be necessary to detemine the phase space coordinates of the beam entering the component and to measure these coordinates on the beam exiting from the component. The beam leaving the component will be acted upon not only by the component but also will be acted upon by ionized residual gas and by its own space charge forces. It is derired that the component ef fects be separated from the ionization and 
spare charge effects. Thus, the measurements on the exiting beam should be made in a vacuum to eliminate ionization effects. Careful planning of the test procedures will be necessary at each stage of the testing. 


\section{ACKNOWLEDGEMENTS}

We wish to acknowledge the valuable assistance from the Magnet Measurement Group at LBL, especially the efforts of D. H. Nelson and M. L. Green, the valuable advice and encouragement of Dr. Edward P. Lee, and the support from Dr. Gene Nolting, Naval Surface Heapon Systems, White Caks Laboratory, Silver Sorings, MD. Additional assistance in coordinating the design interfaces to match the requirements of ATA were received from Gil Cruz of LLNL. Assisting in the work at Brobeck Corporation was J. T. Gunn and J. E. Miller. 
APPENDIX 


\section{ACHROMAT BEND MAGNET DRAWING LIST}

$\begin{array}{ll}\text { Achromat Interface \& Vacuum Test Assembly } & 9501781 \\ \text { Vacuum Transition Flange } & 95 C 1774 \\ \text { Adapter Plate } & 95 C 1775 \\ \text { Bolt Ring } & 95 C 1776 \\ \text { Diaphragm Plate } & 95 B 1777 \\ \text { Adapter Spool } & 95 B 1778 \\ \text { Bearing } & 9581779 \\ \text { Alignment Bar } & 95 B 1780 \\ \text { Downstream Mounting Plate } & 95 B 178 ? \\ \text { Spacer } & 95 B 1783 \\ \text { Stud } & 95 B 1784 \\ \text { Achromat Assembly } & 9511749 \\ \text { Housing } & 95 J 1739 \\ \text { Beam Tube } & 95 C 1752 \\ \text { Quadrupole Assembly } & 95 C 1750 \\ \text { Dipole Assembly } & 95 C 1751 \\ \text { Trim Coil Assembly } & 95 C 1740 \\ \text { Magnet Spacer } & 95 B 1738 \\ \text { Hater Manifold } & 95 C 1743 \\ \text { Flange Ring } & 95 C 1744 \\ \text { Adjusting Sleeve } & 95 C 1745 \\ \text { Retainiing Flange } & 95 B 1746 \\ \text { Retaining Sleeve } & 9581747 \\ \text { Barrier Ring } & 9581748 \\ \text { Focusing Quad } & 9501732 \\ \text { Defocusing Quad } & 9501733 \\ \text { Segment Carrier - Ouad } & 95 C 1736 \\ \text { Dipole } & 9501734 \\ \text { Segnent Carrier - Dipole } & 95 C 1737 \\ \text { Manifold Fitting 1/2" } & 95 B 1741 \\ \text { Manifold Fitting 1/4" } & 95 B 1742\end{array}$


Vernier Steering Magnet Installation

9531728

Coil Assembly

95E 1708

Lock Nut

$95 \mathrm{C} 1710$

Interface Flange

Downstrean Mounting Plite

$95 E 1714$

Manifold Clamp

Supply Manifold

95E 1716

9581717

9501720

Return Manifold

9501721

Magnet Power Input Flange

$95 \mathrm{C} 1723$

Magnet Cooling Inr,ut Flange

95C1724

Water Supply Connuctor

Water Return Connector

$95 B 1725$

Spreader

$95 B 1726$

Magnet Power Teminal

$95 B 1727$

2-Port Exp. Tank

95B1729

Pump-out Spool

Tank Spool $(50 \mathrm{~cm})$

Cover (6" Port)

Coil Spool

Coil $X_{1} \times 5$

Coi) $Y_{1} 2 Y_{5}$

9501769

9501771

9501770

$95 \mathrm{C} 1772$ ?

951707

9501705

9501706

Cooling Coil

9501709

Y Coil Spacer

$95 C 1712$

x Coil Spacer

$95 C 1713$

Cover (12" Port)

95C1773

Manifold Fitting $1 / 2^{\prime \prime}$

9581718

Manifold Fitting 3/16"

9581719 